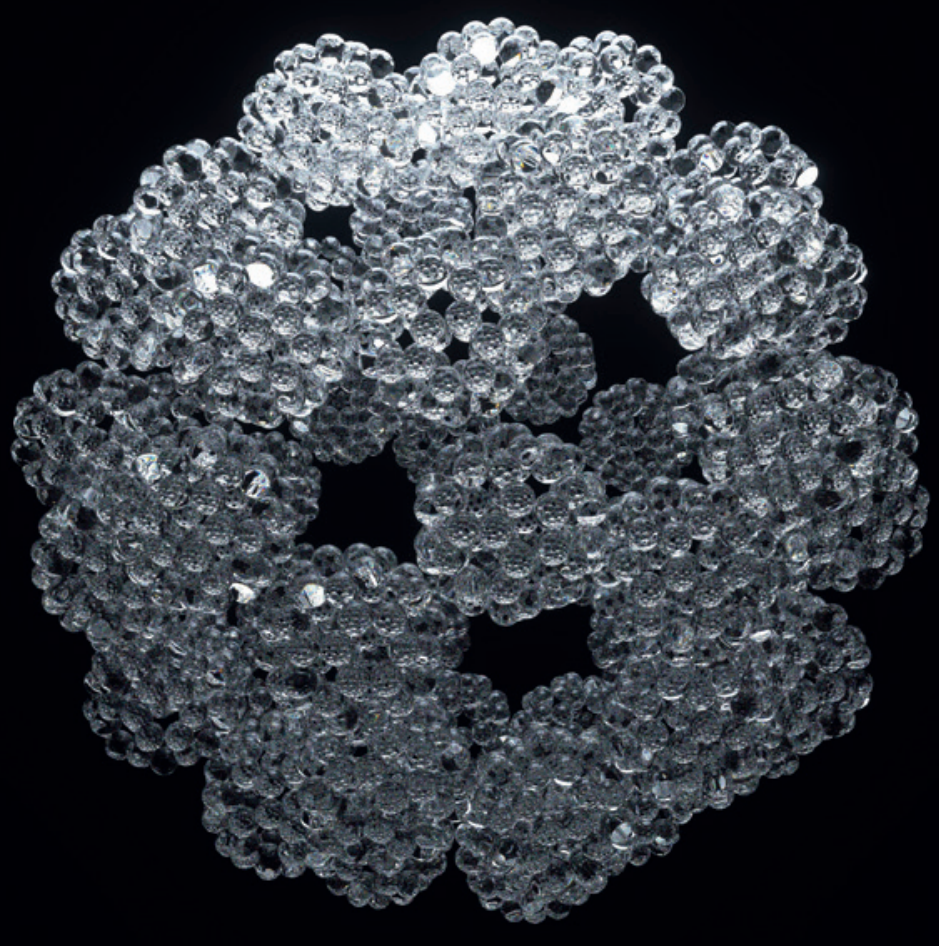

Joseph B. Natowitz and Christian Ngô

\title{
Our Nanotechnology Future
}


Our Nanotechnology Future 


\title{
Atlantis Advances in Nanotechnology, Material Science AND ENERGY TECHNOLOGIES
}

\author{
Series Editor: Dr. Christian Ngô
}

ISSN: $235^{2-0655}$

The aim of "Atlantis Advances in Nanotechnology, Material Science and Energy Technologies" is to publish high quality manuscripts giving an up-todate and clear view on topical scientific contents in nanotechnology, material sciences and energy technologies.

These three fields evolve rapidly and their understanding is essential with regard to contemporary science and as well as in the context of everyday life. Nanotechnology is a fast growing science and a technological field with applications in numerous areas such as materials, health, electronics, information processing, defence and security, catalysis, sensors, food, cosmetics and many more. The results of material sciences are the basis for any object around us, they are omnipresent in human life. Mastering materials and processes is therefore crucial. In particular, research on microscopic understanding is essential to develop models predicting the properties of new materials and structures. The final goal is to be able to predict macroscopic properties of materials from their microscopic properties. Finally, energy technologies enfold a complex area where each technological advance has to be weighed against economical, environmental, political and sociological constraints. Energy is closely linked to economic development and, more generally speaking, to everyday life.

As nanotechnology, materials science and energy technologies are closely interconnected, this series offers the reader both, highly specialized monographs as well as easy-to-grab overviews. Each publication focuses on one of the fields. At the same time, it is highly relevant to explore their interconnections and to include interdisciplinary approaches.

All book proposals submitted to this series are being reviewed by key experts before publication and each book focuses on a certain field putting it into perspective with its implications at the economic and societal level. 


\section{Our Nanotechnology Future}

Christian Ngô and Joseph B.Natowitz

Atlantis Press / Amsterdam University Press 
Cover illustration: (ㄷ) 2010 James Coleman

Cover design: Coördesign, Leiden

Lay-out: Newgen/Konvertus

Amsterdam University Press English-language titles are distributed in the US and Canada by the University of Chicago Press.

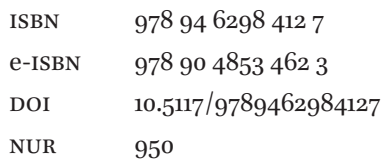

\section{(c) $(i)($ ) $(9)$}

Creative Commons License CC BY NC ND

(http://creativecommons.org/licenses/by-nc-nd/3.o)

CC All authors / Atlantis Press B.V. / Amsterdam University Press B.V., Amsterdam, 2017

Some rights reserved. Without limiting the rights under copyright reserved above, any part of this book may be reproduced, stored in or introduced into a retrieval system, or transmitted, in any form or by any means (electronic, mechanical, photocopying, recording or otherwise). 


\section{Contents}

Introduction

I. Nanoscience and nanotechnology $\quad 15$

The size domain of nanoscience and nanotechnology $\quad 15$

$\begin{array}{ll}\text { Nanotechnology in the past } & 17\end{array}$

$\begin{array}{ll}\text { Intellectual foundations of nanotechnology } & 18\end{array}$

$\begin{array}{ll}\text { Can we see atoms? } & 19\end{array}$

The scanning tunneling microscope 20

$\begin{array}{ll}\text { The atomic force microscope } & 23\end{array}$

$\begin{array}{ll}\text { Manipulating atoms } & 24\end{array}$

$\begin{array}{ll}\text { Summary } & 26\end{array}$

II. The Quantum world $\quad 27$

Classical versus quantum mechanics $\quad 27$

$\begin{array}{ll}\text { Wave-particle duality } & 28\end{array}$

Determinism versus a probabilistic approach $\quad 28$

Measurement 30

Quantization 31

Heisenberg uncertainty principle $\quad 32$

Quantum numbers $\quad 34$

Spin - an intrinsic property $\quad 34$

Fermions and bosons $\quad 35$

Quantum tunneling $\quad 36$

Summary $\quad 38$

III. The mesoscopic world 40

The forces of nature $\quad 40$

The role of size $\quad 40$

Surface versus volume $\quad 41$

Surface tension $\quad 43$

Nanofluids $\quad 43$

Heat transfer $\quad 44$

Chemical bonding $\quad 44$

Intermolecular forces $\quad 45$ 
$\begin{array}{ll}\text { Semi-classical approaches } & 47\end{array}$

Summary $\quad 48$

IV. Nanomaterials and nanostructures $\quad 49$

Classifying nanomaterials $\quad 49$

Nanostructuration $\quad 5^{1}$

Dendrimers $\quad 55$

Hybrid organic-inorganic nanomaterials $\quad 57$

Nanocomposites $\quad 58$

$\begin{array}{ll}\text { Smart materials } & 59\end{array}$

$\begin{array}{ll}\text { Summary } & 60\end{array}$

V. Natural nanomaterials $\quad 61$

$\begin{array}{ll}\text { Nanomaterials in nature } & 61\end{array}$

$\begin{array}{ll}\text { Self-cleaning surfaces } & 61\end{array}$

$\begin{array}{ll}\text { Reversible adhesives } & 62\end{array}$

$\begin{array}{ll}\text { Lightweight materials } & 64\end{array}$

Manipulating light at the nanoscale $\quad 65$

$\begin{array}{ll}\text { Fibers stronger than steel } & 67\end{array}$

$\begin{array}{ll}\text { Low-friction materials } & 69\end{array}$

$\begin{array}{ll}\text { Multiscale structures } & 69\end{array}$

$\begin{array}{ll}\text { Summary } & 69\end{array}$

VI. Nanofabrication $\quad 71$

Top-down and bottom-up approaches $\quad 71$

$\begin{array}{ll}\text { Lithography } & 72\end{array}$

Deposition at the nanoscale $\quad 78$

Polishing, etching, patterning $\quad 82$

Summary $\quad 83$

$\begin{array}{lr}\text { VII. King carbon } & 84\end{array}$

$\begin{array}{lr}\text { Fullerenes } & 84\end{array}$

$\begin{array}{ll}\text { Graphene } & 88\end{array}$

Carbon nanotubes $\quad 90$

$\begin{array}{ll}\text { Summary } & 94\end{array}$

$\begin{array}{lll}\text { Part } 2 & \text { Applications of nanotechnology } & 97\end{array}$

VIII. Health Diagnostics $\quad 99$

$\begin{array}{ll}\text { Major diseases } & 100\end{array}$ 
$\begin{array}{ll}\text { Diagnosis } & 101\end{array}$

$\begin{array}{ll}\text { Imaging } & 101\end{array}$

$\begin{array}{ll}\text { In-Vitro Diagostics } & 103\end{array}$

$\begin{array}{ll}\text { Biosensors } & 104\end{array}$

$\begin{array}{ll}\text { Biochips } & 106\end{array}$

$\begin{array}{ll}\text { Labs-on-chips } & 108\end{array}$

Cells-on-chips $\quad 110$

Summary 111

IX. Therapeutics 112

Drug delivery 112

Delivery routes $\quad 113$

Drug carriers $\quad 114$

Nanoparticles and drug delivery $\quad 116$

$\begin{array}{ll}\text { Summary } & 118\end{array}$

$\begin{array}{ll}\text { X. Regenerative medicine } & 120\end{array}$

Biomaterials $\quad 120$

Cell therapy $\quad 121$

Implants $\quad 122$

Dentistry $\quad 124$

Nanosurgery $\quad 126$

$\begin{array}{ll}\text { Summary } & 127\end{array}$

$\begin{array}{lr}\text { XI. The Food Chain } & 128\end{array}$

$\begin{array}{ll}\text { Feeding people } & 128\end{array}$

Agricultural Production $\quad 130$

Food processing $\quad 131$

Packaging 133

Transportation 134

Summary $\quad 135$

XII. From microelectronics to nanoelectronics $\quad 136$

$\begin{array}{lr}\text { Transistors } & 136\end{array}$

Moore's law 137

Technology nodes 139

Memories 139

Smaller, faster and cheaper $\quad 143$

$\begin{array}{ll}\text { Summary } & 144\end{array}$ 
$\begin{array}{lr}\text { XIII. Quantum nanoelectronics } & 145\end{array}$

Towards few-electron electronics $\quad 145$

$\begin{array}{ll}\text { Coulomb blockade } & 146\end{array}$

The single electron transistor $\quad 149$

Quantum dots $\quad 151$

Spintronics $\quad 154$

Nanophotonics $\quad 157$

Confining and Controlling light $\quad 15^{8}$

Photonic crystals $\quad 15^{8}$

$\begin{array}{ll}\text { Plasmonics } & 161\end{array}$

$\begin{array}{ll}\text { Metamaterials } & 162\end{array}$

$\begin{array}{ll}\text { Summary } & 163\end{array}$

$\begin{array}{ll}\text { XIV. Molecular electronics } & 164\end{array}$

$\begin{array}{ll}\text { Electronic conduction } & 164\end{array}$

Difficulties $\quad 165$

Molecular wires $\quad 166$

Molecular diodes and transistors $\quad 168$

$\begin{array}{ll}\text { Conductive polymers } & 169\end{array}$

Self-assembled monolayers $\quad 171$

Summary 171

$\begin{array}{lr}\text { XV. Nanocatalysis } & 173\end{array}$

$\begin{array}{ll}\text { Catalysts make life easier } & 173\end{array}$

Nanocatalysts for chemical reactions $\quad 175$

$\begin{array}{ll}\text { Enzymes speed up biological reactions } & 177\end{array}$

$\begin{array}{lr}\text { Designing nanocatalysts } & 178\end{array}$

$\begin{array}{ll}\text { Summary } & 179\end{array}$

$\begin{array}{lr}\text { XVI. Energy production } & 181\end{array}$

$\begin{array}{ll}\text { Fossil fuels } & 182\end{array}$

Renewable energies $\quad 184$

Energy storage $\quad 185$

$\begin{array}{ll}\text { Electricity } & 187\end{array}$

Hydrogen $\quad 187$

$\begin{array}{ll}\text { Fuel cells } & 188\end{array}$

Thermoelectricity $\quad 189$

Nuclear energy 190

Summary 192 
$\begin{array}{lr}\text { XVII. Housing } & 193\end{array}$

Outside the building 193

Inside the buildings $\quad 195$

$\begin{array}{ll}\text { Summary } & 200\end{array}$

XVIII. Nanotechnology in automobiles 201

Bodywork 201

Interior of the car $\quad 205$

Chassis and tires $\quad 205$

Power train $\quad 206$

$\begin{array}{ll}\text { Electronics } & 207\end{array}$

Batteries and supercapacitors $\quad 207$

$\begin{array}{ll}\text { Summary } & 209\end{array}$

$\begin{array}{lll}\text { XIX. Defense and security } & 211\end{array}$

Defense 211

Homeland security $\quad 216$

$\begin{array}{ll}\text { Summary } & 223\end{array}$

XX. Nanotoxicity $\quad 224$

Hazard and risk $\quad 224$

Nanomaterials and nanoparticles $\quad 225$

$\begin{array}{ll}\text { Nanoparticle sources } & 226\end{array}$

Nanoparticle exposure $\quad 226$

Toxicity of nanoparticles $\quad 227$

Natural nanoparticles $\quad 231$

Anthropogenic nanoparticles $\quad 233$

Engineered nanoparticles $\quad 234$

Summary $\quad 236$

$\begin{array}{ll}\text { Notes } & 239\end{array}$

List of illustrations' copyright and licensing $\quad 242$

Short bibliography $\quad 244$ 



\section{Introduction}

Nanotechnology is pervasive in contemporary life. With applications in food, electronics, medicine, cosmetics, catalysis, construction, defense etc. Many of natural materials around us have a nanostructure that determines their behavior. Nanoscience and nanotechnology generally deal with objects that have one or more dimensions in the range of 1-100 $\mathrm{nm}$. This range is arbitrarily fixed by convention and sometimes must be expanded. Modern nanoscience and nanotechnology really started about three decades ago when it was demonstrated that it is possible to observe and manipulate nano-objects. Before that, most manufactured nanomaterials were films or coatings with thicknesses in the range of 1-100 $\mathrm{nm}$.

Nano-objects can be built from elementary components (atoms or molecules), or by breaking down or carving bulk materials using different methods. The first approach is usually called "bottom-up" while the second one is termed as "top-down". Nanocharacterization techniques play a major role in these processes because they can be used to monitor the properties of these objects in a size range spanning the region between the microscopic world that encompass atoms, small molecules and nuclei- where the physics is governed by quantum phenomena - and the macroscopic world that can be described by classical theories such as classical mechanics. A good understanding of phenomena at the nanoscale, affords us the ability to boost the properties of manmade materials by tailoring nanomaterials for specific purposes. The properties manifested at the nanoscale level can be harnessed to provide remarkable new materials and capabilities.

Although the rate of development of nanotechnology today is smaller than was foreseen a decade ago, it increases regularly in an irreversible way. This short book is intended to give the reader a flavor of this expanding domain and its applications The book is divided in two parts. Part 1 presents the fundamental tenets of nanoscience and nanotechnology. Part 2 discusses current and future applications that have, or will have, a major influence on our lives.

Because a sharp rise in the use of nanotechnology in commercial products is expected in the future, the question of the possible risk that nanoparticles pose to human health is an important issue. Although humans have always been faced with the presence of natural nanoparticles, the manufacturing and use of engineered nanoparticles should be carefully investigated as far as the risk to living species is concerned. The risk must be carefully balanced against the advantages that can be obtained through nanoparticle usage. 

Part 1 Nanotechnology basics 



\section{Nanoscience and nanotechnology}

\section{The size domain of nanoscience and nanotechnology}

Nanoscience is the study of the performance of ultra-small autonomous structures. The nanoscience domain is typically viewed as having dimensions ranging between $1 \mathrm{~nm}$ and $100 \mathrm{~nm}$. One nanometer $(1 \mathrm{~nm})$ is one billionth of a meter and one millionth of a millimeter. It is a very small distance. in fact, it is a length comparable to the size of individual atoms. One gold atom has a diameter of $\approx 0.144 \mathrm{~nm}$. Seven gold atoms placed in a line and touching would extend for about $1 \mathrm{~nm}$. For comparison, the cross sectional diameter of a strand of a human DNA molecule is about $2.5 \mathrm{~nm}$, the diameter of a blood cell is about 7,000 $\mathrm{nm}$ and the diameter of a human hair is in the range of 80,000 to $100,000 \mathrm{~nm}$.

If we could shrink all distances of our macroscopic world by one billion, one meter would become $1 \mathrm{~nm}$. In this case the distance between the earth and the moon $(\approx 360$,00o $\mathrm{km})$ would become $36 \mathrm{~cm}$ and the distance between the earth and the sun $(\approx 150,000,000 \mathrm{~km})$ would become $150 \mathrm{~m}$.

In figure 1 we list some common units of length ranging from one meter to one femtometer, $10^{-15}$ meter. The nuclei of atoms have diameters in the range of 1 to 20 femtometers.

The conventional delineation of $1 \mathrm{~nm}$ to $100 \mathrm{~nm}$ for the nanoscience domain is useful, but should not be considered as a rigorous definition. Structures with larger dimensions can, in some cases, manifest phenomena belonging to this domain. It is important to investigate the different phenomena taking place on this size scale because they are often size dependent and different from those observed in our macroscopic world. Understanding and learning to model, simulate and manipulate nanoscale matter is a necessary step toward providing a firm underpinning for nanotechnology. Nanotechnology deals with the practical techniques of design, manufacturing and applications of nanostructured materials and devices. Nanotechnology has applications in many fields, including health, food, materials science, manufacturing, energy and chemical processes, among others. Nanotechnology is rapidly becoming more and more important in improving the materials and objects we routinely use in our daily lives. 


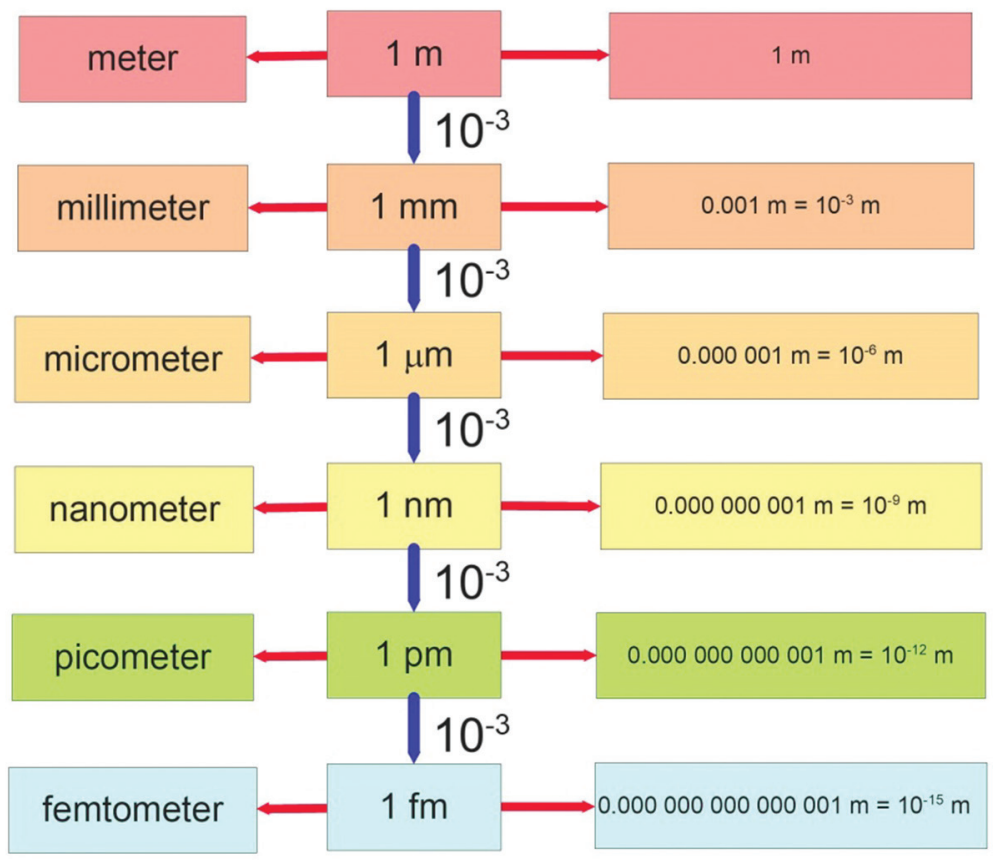

Figure 1. Length units descending from 1 meter to 1 femtometer. Each unit represented is separated from the adjacent units by a factor 1,000 .

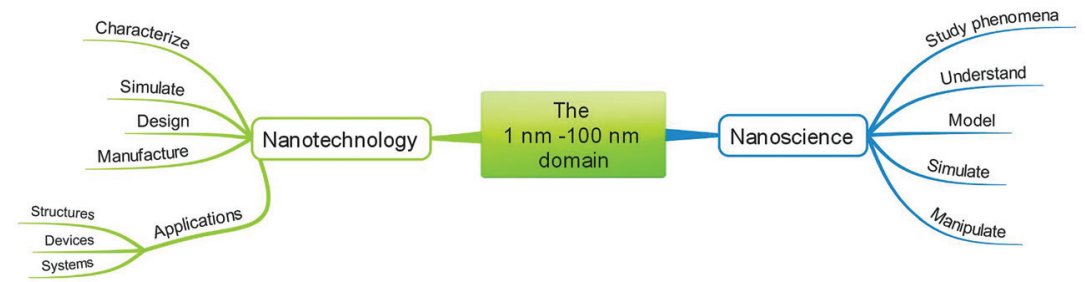

Figure 2. Some keywords of nanoscience ad nanotechnology.

Figure 2 provides a schematic view of the components constituting nanoscience and nanotechnology.

Nanomaterials need not be man-made. A number of natural nanostructured material exist. These occur in animals, in plants and in minerals. Examples include structures on the eye of a moth which increase the moth's ability to see in the dark, surface structures on the leaves of a lotus plant which make them water repellent and nano-structured clays which are often employed as additives to modify material properties. 


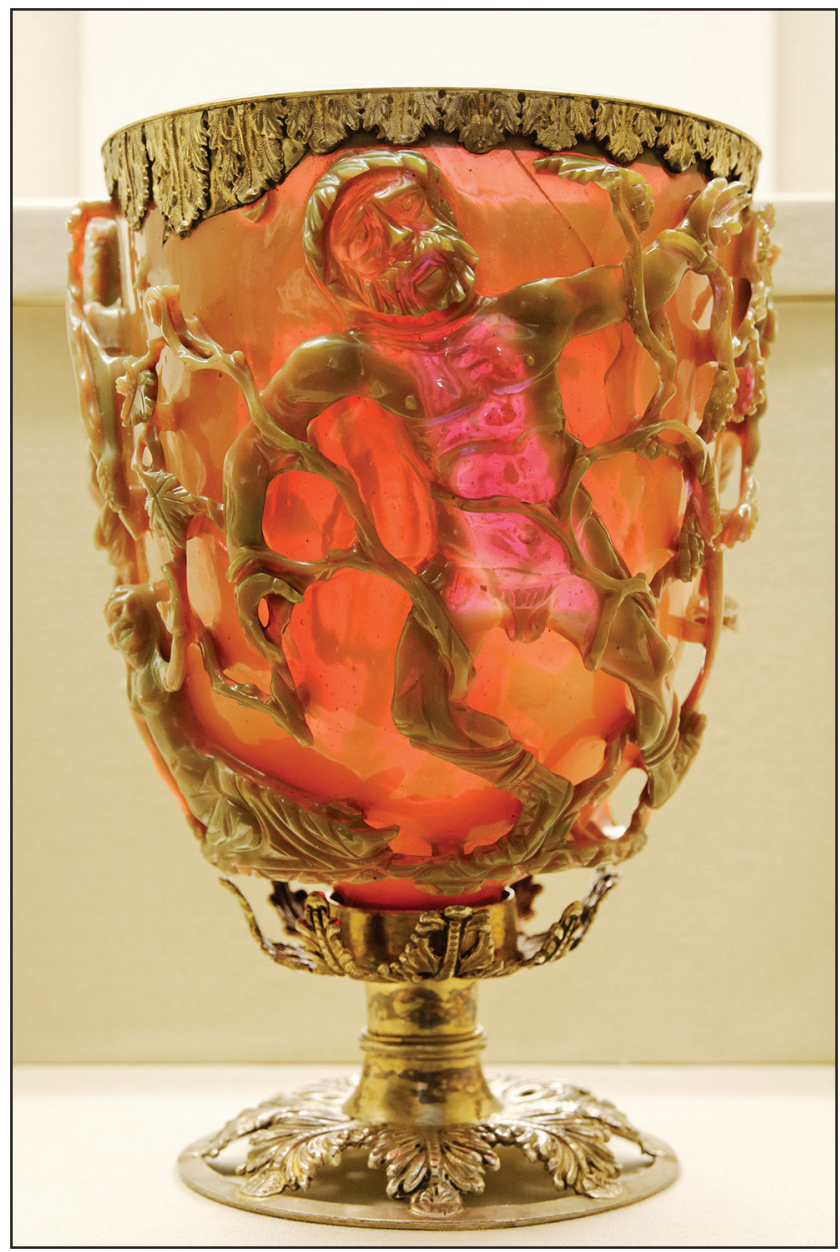

Figure 3. The Lycurgus cup exhibited at the British museum. It was manufactured by the Romans around the 4th century AD. The color varies. This dichroic property comes from the presence of nanoparticles, which were produced during the manufacturing of the glass.

\section{Nanotechnology in the past}

The first human created nanostructures were not understood to be such. For example the "Lycurgus cup", presently in the British Museum in London, is a glass cup manufactured in Rome around the fourth century A.D., representing a scene from mythology, it was made from dichroic glass, glass which undergoes color changes in different lighting conditions. Illuminated from the outside, the cup looks green. Illuminated from the inside the cup appears ruby red except for the figure of the king which takes on a purplish hue (figure 3 ). 
The dichroism of the glass is due to nanosized particles (up to $100 \mathrm{~nm}$ ) embedded in the glass. These nanoparticles are of silver and gold. There is also a small amount of copper nanoparticles. The absorption and the scattering of light by these nanoparticles determines the color which is observed. When this cup was made, nothing was known about nanotechnology, but the manufacturer succeeded in creating this unique object.

Nanoparticles are also present in other objects produced in the Middle Ages. In the 1850 s the British physicist and chemist Michael Faraday proposed that the observed color variations in stained glass windows could be attributed to variations in size of the clusters of metal atoms embedded in them. Some Chinese porcelains produced in the same time period contain 20-6o nm nanosized gold particles.

\section{Intellectual foundations of nanotechnology}

On December 1959, Richard Feynmann (Nobel Prize in Physics, 1965) gave a visionary lecture entitled "There's plenty of room at the bottom". In that lecture he stated his belief that it should be possible to manipulate atoms on an atomic scale and arrange them in desired patterns using nanoscale machines. At the end of his talk, he challenged his listeners to accomplish two tasks. The first was to build an electric motor smaller than $0.4 \mathrm{~mm}$ on a side. The second was to scale letters small enough to be able to write the entire Encyclopedia Britannica on the head of a pin. This requires being able to write a standard printed page on a surface 25,000 times smaller than that page. To do so each letter must have a size of about $9 \mathrm{~nm}$. He offered $\$ 1,000$ prizes to the first people able to solve either of these challenges.

The first task was achieved in November 1960 by William McLellan, who manufactured a motor weighing $2500 \mu \mathrm{g}$ and having 13 parts which could function at $2000 \mathrm{rpm}$. Feynman was a bit disappointed because it was constructed with conventional tools and did not employ any new technology. The second challenge was met in 1985 by Tom Newman, a Stanford graduate student, who used an electron beam to inscribe the first page of Charles Dickens' "A Tale of Two Cities" on the head of the pin. Because most of the area of the head of the pin remained empty, he later actually had a hard time finding the written text on the pin.

Today Feynman's lecture is generally considered as the starting point of the nanotechnology story. The word "nanotechnology" itself was first 
introduced in 1979 by Norio Taniguchi, a Japanese scientist, to describe the control of semiconductor processes on the nanometer scale.

\section{Can we see atoms?}

Around 450 BC, Leucippus and Democritus, two Greek philosophers, suggested that matter was made of very small particles, invisible to a naked eye. A convincing verification of this concept came in 1805 , thanks to the work of English chemist John Dalton. The macroscopic matter we see around us is indeed composed of individual units we call atoms. Atoms are the smallest units of a chemical element which retain the characteristics of that element. While we have known for over two centuries that matter is made up of atoms, our understanding of the nature and structure of atoms has evolved greatly during that period.

"Seeing is believing." is a credo firmly ingrained in the human psyche. However there are many objects which the unaided human eye is not capable of seeing. Atoms are in this realm. We must rely on instruments much more sensitive than our eyes to detect or see individual atoms. In figure 4, we show examples of objects of different sizes and indicate the different length scales, each separated by a factor 1,000, associated with these objects. For example, we can easily see people with our eyes. Small insects may have a size of a few millimeters or centimeters and a magnifying glass may be needed to observe them better. Red blood cells with a size on the order of $7-8 \mu \mathrm{m}$ can be observed with an optical microscope. Silicon wires of an integrated circuit, such as shown at the far right in figure 4, have widths of several nanometers and can be observed with electron microscopes or scanning tunneling microscopes.

Electron microscopes are instruments that employ accelerated electrons to observe small objects. The physical laws governing the propagation of these electrons lead to size resolutions $\sim 100,000$ times better than those achievable with visible light. With this higher the electron microscope can "see" extremely small objects. Figure 5 illustrates the kinds of images obtainable using different electron microscopy techniques.

The spatial resolution of a scanning electron microscope can be about 1 $\mathrm{nm}$ to $20 \mathrm{~nm}$ depending on the instrument. The transmission electron microscope has a better spatial resolution and can reach $0.05 \mathrm{~nm}$. 


\section{Different length scales}
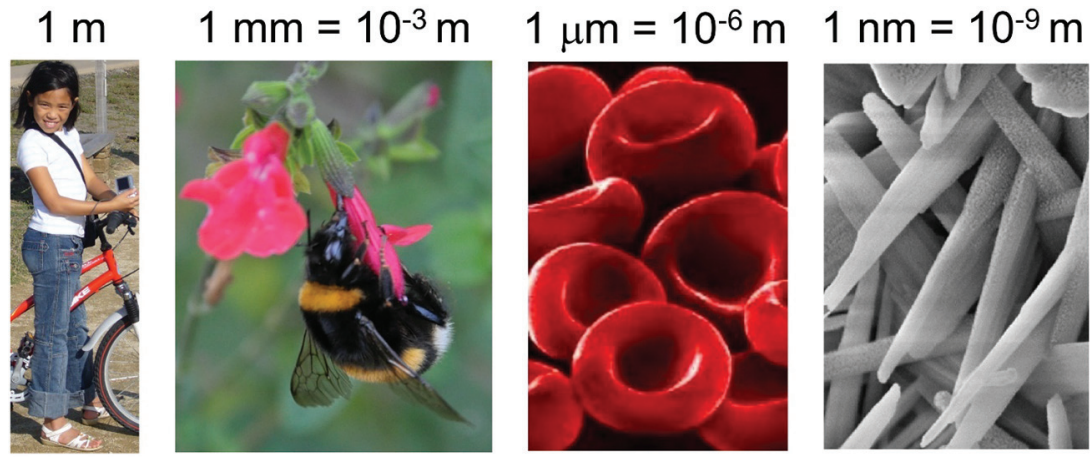

Figure 4. Examples of objects of different sizes.

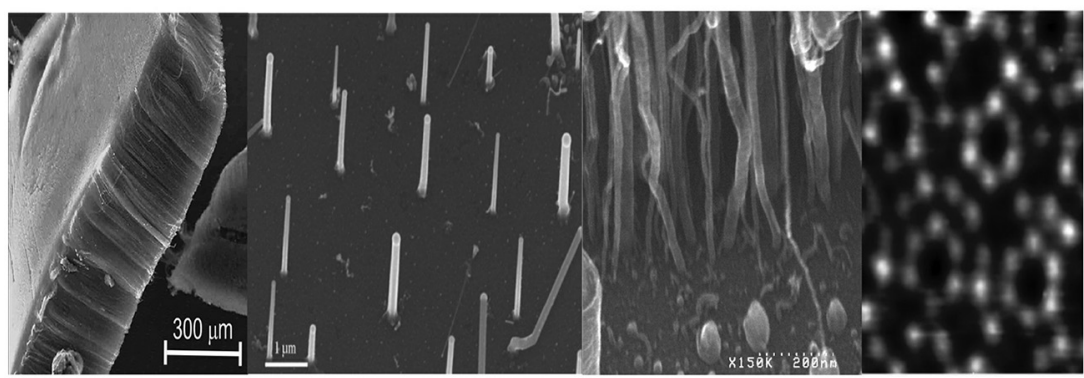

Figure 5. Images showing objects of various sizes taken using different microscopic techniques. From left to right - a carbon nanotube monolayer forest as seen by a standard electron microscope, a scanning electron microscope image showing silicon nanowires synthesized by vapor-liquid-solid techniques with diameters in the range $30-100 \mathrm{~nm}$, a scanning electron microscope image of carbon nanotubes grown on a silicon substrate in a reactor at the CEA, an STM image of a silicon crystal with the surface oriented along the (111) plane. In the latter case, the bright dots correspond to individual Si-atoms at the surface. All these images are courtesy of CEA/LETI (France).

\section{The scanning tunneling microscope}

An important breakthrough in the observation of the constituents of matter was made in 1981 when Gerd Binnig and Heinrich Rohrer, researchers at IBM-Zurich in Switzerland, invented the Scanning Tunneling Microscope (STM). With this device, it became possible to see (after computer processing) individual atoms on an electron microscope screen and, furthermore, to manipulate and arrange these individual atoms. In 1986 these two scientists received the Nobel Prize in Physics for this achievement. 


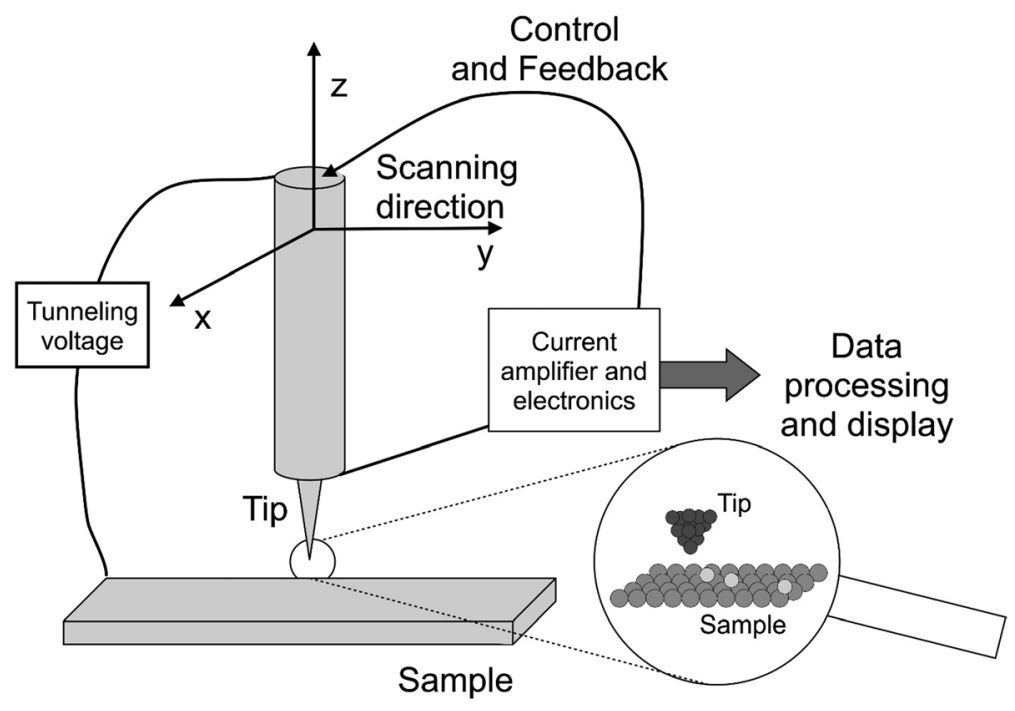

Figure 6. Schematic diagram of a scanning tunneling microscope.

The principle of the STM is shown in figure 6. A conducting tip is brought to a very small distance (a distance below $1 \mathrm{~nm}$ ) from the surface of the sample being scanned. Materials used for the tip can be tungsten, a platinum-iridium alloy and even gold. The sample can be a conductor or a semi-conductor. The important interaction is between the outermost atom on the tip and an atom on the surface of the sample. When the tip and the surface are very close to each other, electrons can tunnel from the sample to the tip or vice versa depending upon the sign of the voltage difference between the tip and the sample. This exchange of electrons is not a classical phenomenon, but a quantum mechanical phenomenon. Quantum phenomena are discussed further in Chapter 2. The tunneling current which is measured depends on the tip position, on the applied voltage, and on the local density of electronic states of the sample. The tunneling current varies exponentially with the distance separating the tip from the surface. A $10 \%$ decrease in this distance $(\approx 1 \mathrm{~nm})$ typically increases the current by an order of magnitude and vice versa. The range of distance separating the tip from the surface of the sample typically varies from 0.4-0.7 $\mathrm{nm}$. A lateral resolution of about $0.1 \mathrm{~nm}$ and a depth resolution of $0.01 \mathrm{~nm}$ can be obtained with the STM.

A STM can operate in a wide range of temperatures from near zero Kelvin $\left(\approx-273^{\circ} \mathrm{C}\right)$ up to a few hundred degrees Celsius. The position of the tip with respect to the surface has to be perfectly controlled mechanically. This is done using piezoelectric mechanisms. 


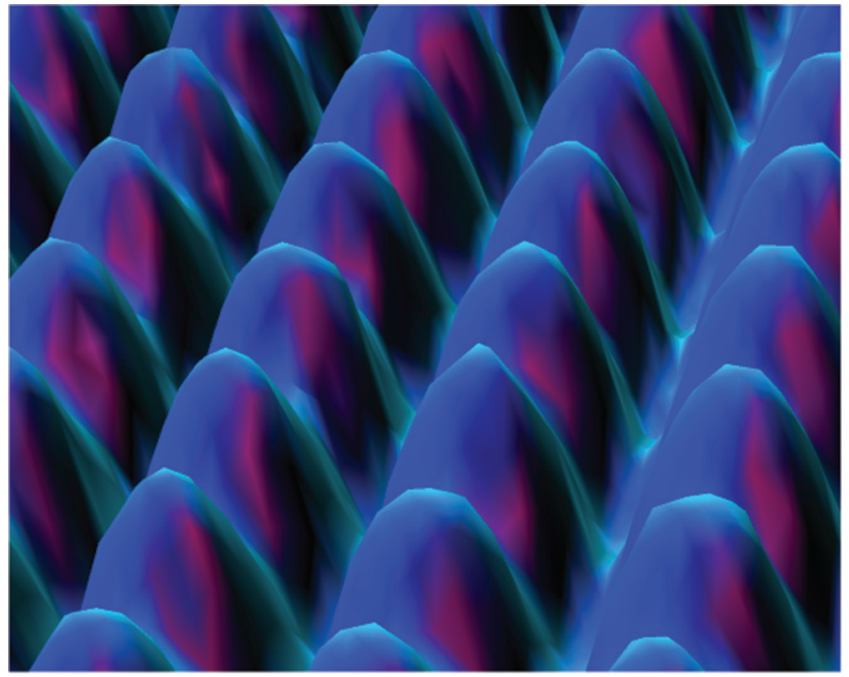

Figure 7. STM image of the surface of a nickel crystal. Image originally created by IBM Corporation.

\section{Constant-height mode}

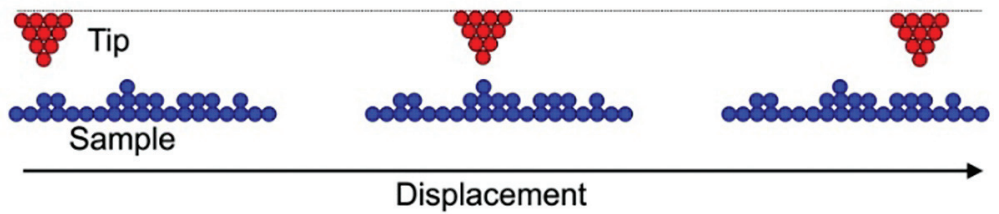

\section{Constant-current mode}

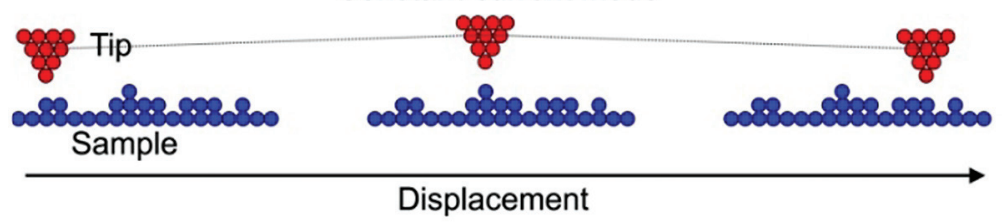

Figure 8. Schematic illustration of the two operating modes of the STM.

The measurement of the local density of the electron quantum states allows the imaging of the spatially-resolved electron density of the sample. Computer processing of that information produces an image of surface atoms on a screen. An example of such an image is that in figure 7 showing the regular arrangement of the nickel atoms on the surface of a nickel crystal.

As shown in figure 8, a STM can be operated in two different modes,: the constant-height mode and the constant-current mode. In the 
constant-height mode, the tip remains at a fixed height and scans the sample while remaining in a horizontal plane. This mode can quickly scan the sample, but requires a smooth sample surface. In the constant-current mode a feedback loop is used to adjust the distance between the tip and the surface of the sample in order to keep the tunneling current constant. This mode is slower than the preceding one, but is able to scan irregular surfaces.

\section{The atomic force microscope}

One of the shortcomings of the STM is that it can only study conductive or semi-conductive surfaces. In 1986, Gerd Binnig, Christoph Gerber and Calvin Quate invented the Atomic Force Microscope (AFM) which can be used on insulating surfaces also. The principle, shown in figure 9, is to use a tip attached to a cantilever (flexible arm). When this tip is moved over the surface of the sample the atomic forces attract or repel the tip as the tip moves along. The motion of the tip induces a motion of the cantilever. A laser beam directed at the cantilever is reflected and the angle of reflection of this beam is measured (see figure 9). From this information, it is possible to build an image of the surface as the tip moves.

There are some basic differences between an AFM and a STM. With an AFM the tip makes a gentle direct contact with the scanned surface while the tip of a STM does not make direct contact and just measures the tunnel current existing between the tip and the surface. The AFM resolution turns out to be better than the STM resolution.

An AFM is well suited for nanotechnology studies because it gives better surface measurements than a STM and can be operated in a wider variety of environments. It can be used with conductors, semiconductors and insulators.

An AFM can be operated in several modes. In the dynamic mode, the cantilever vibrates around a given frequency and the tip oscillates with an amplitude of a few nanometers close to the surface of the sample. The long range forces, such as Van der Waals forces, affect the frequency of oscillation and a feedback loop is used to keep the frequency of oscillation constant by changing the distance separating the tip from the surface of the sample. In this way a topological mapping of the surface can be made. In the intermittent or tapping mode, the oscillations of the cantilever are much larger and there is an intermittent contact between the tip and the surface. This technique is particularly suitable when a thin layer of liquid 


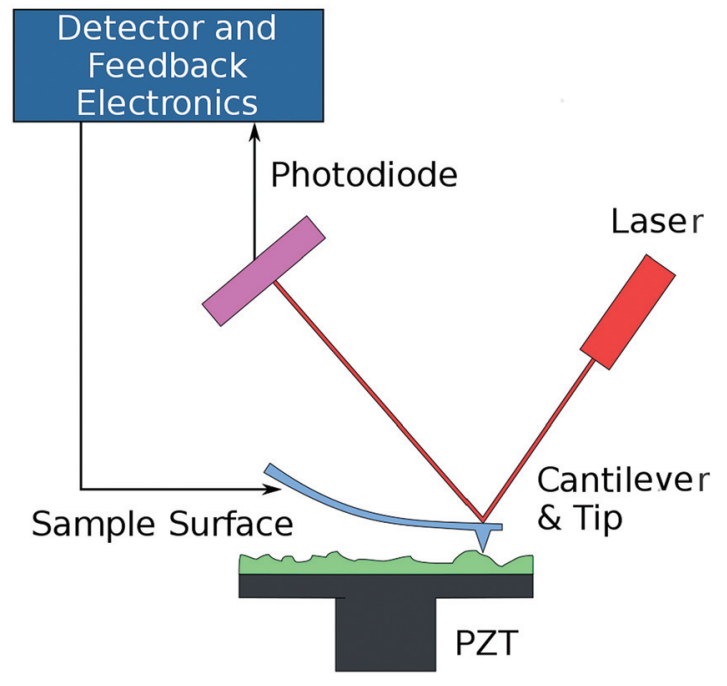

Figure 9. Schematic of an atomic force microscope with optical detection of the deflection of the microcantilever. Image from Wikimedia Commons (http://commons.wikimedia.org). Author Grzegorz Wielgoszewski.

has developed on the surface of the sample because of the environmental conditions. It is the method most often employed in AFM measurements. In the contact mode, the tip touches the surface. The electrons of the atoms repel it and the cantilever is deflected.

\section{Manipulating atoms}

Using a STM it is possible not only to see atoms but also to move individual atoms from one point on the surface to another. In 1989, Don Eigler, an IBM researcher, using a STM, picked up and moved an individual atom for the first time. He moved a xenon atom back and forth 3 times in order to check reproducibility. In his lab notebook Eigler wrote "Did it," "Did it" and "Did it again! 3 in a row". After this crucial breakthrough, in November 1989, Eigler succeeded in arranging 35 atoms of xenon on the surface of a nickel crystal to write the word "IBM" (figure 10). Eigler claimed that once the atom-moving process was under control, the biggest challenge was "remembering how to spell IBM".

Atoms moved and positioned on the surface of a crystal are often called adatoms $s^{1}$. The move can be done manually or with the aid of a computer. An autonomous atom assembler has been recently developed by researchers from the US Center for Nanoscale Science and Technology. It provides 


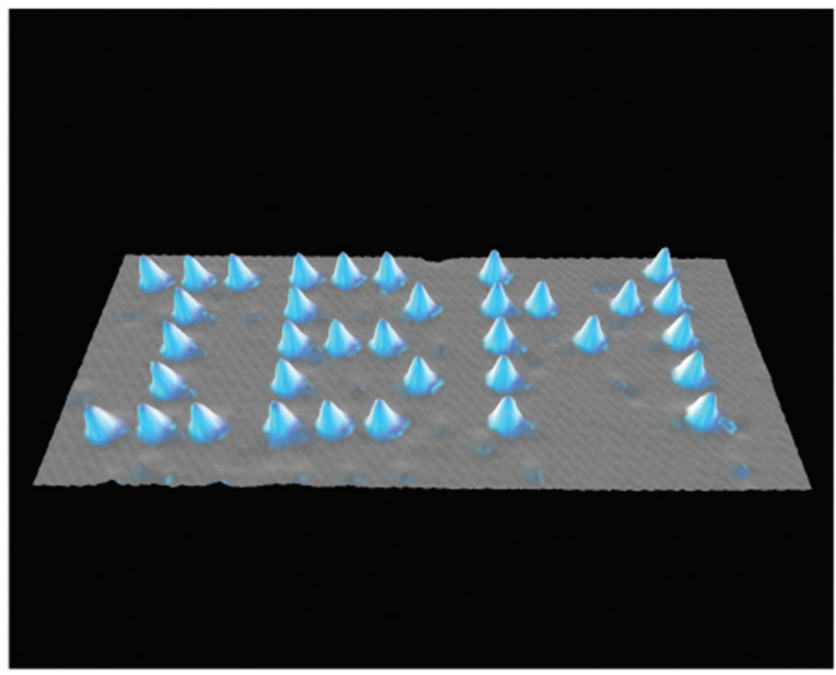

Figure 10. Atoms of xenon arranged on a nickel crystal surface to spell IBM. Courtesy of IBM company (www.almaden.ibm.com).

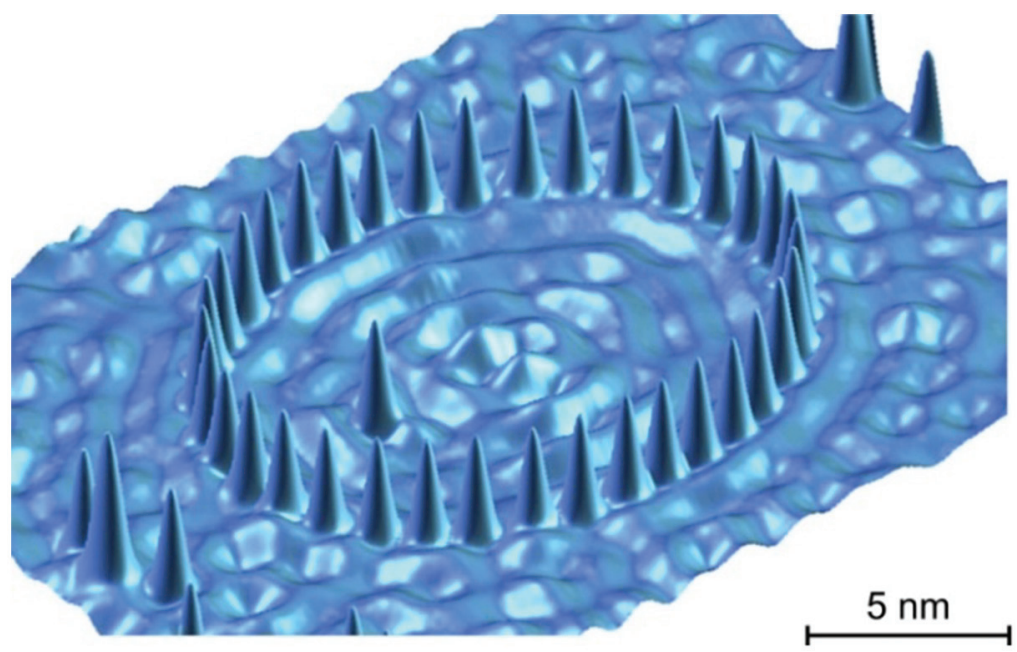

Figure 11. Image of an elliptical quantum corral built using the autonomous atom assembler; Co atoms were deposited at sub-monolayer coverage on $\mathrm{Cu}(111)$ at $7 \mathrm{~K}$ in ultra high vacuum and subsequent STM measurements were performed at a $4.3 \mathrm{~K}$ sample temperature. J.A. Stroscio, R.J. Celotta, S.R. Blankenship and F.M. Hess, http://www.nist.gov/cnst/epg/atom_manipulation_stm. $\mathrm{cfm}$. Image from Wikimedia Commons (http://commons.wikimedia.org).

the ability to assemble a desired nanostructure from atoms initially randomly distributed on the surface of a crystal. The autonomous atom assembler is a dedicated instrument based upon an STM with upgraded hardware 
and software components. Figure 11 shows the result of the manipulation of cobalt adatoms on a copper surface to form a closed quantum structure (quantum corral) in which electrons can be trapped. The geometry of this structure defines new energy levels in this region of the surface. Such a structure, made to measure, may have important applications in the future.

\section{Summary}

It is now possible to routinely see and move atoms. This represents an important technological breakthrough and opens a wide number of science and technological applications. The main issue is to be able to work at the nanoscale at a pace suffiency large to quickly obtain objects useful in the macroscopic word and a competitive cost.

The ability to see details in the nanosized range is necessary and essential for all developments of nanotechnology because it allows to control nanotechnology research developments and manufacturing processes. The possibility to see details, at the scale of the nanometer or better, allows a rational development of nanomaterials by giving the possibility to understand the mechanisms involved rather than developing materials using trial and error methods as it was done in the past.

An adatom is an additional atom lying on the surface of a crystal. This word comes from the contraction of the expression "adsorbed atom". An adatom can be viewed as the opposite of a surface vacancy in which an atom has disappeared. 


\section{The Quantum world}

The motion of macroscopic objects such as soccer balls, vehicles and planets can be well described using classical mechanics. However, classical mechanics is inadequate to describe the movements or the structure of microscopic objects such as atoms, molecules or nuclei. To properly describe the properties of microscopic objects, the theory of quantum mechanics was developed. While quantum mechanics can be applied to macroscopic objects, it turns out that for objects of large dimensions the quantum mechanical laws evolve naturally into classical mechanics. In general, solving a problem in quantum mechanics is far more difficult than solving it in classical mechanics. Therefore, the laws of classical mechanics are employed when they can be applied safely.

As we descend to the nanoscale, the applicability of classical mechanics gradually diminishes. This does not occur at the same time for all of the variables describing the system, but below a certain size, quantum effects will become important. In this chapter, we briefly review some basic features of quantum mechanics. In the next chapter some of the consequences for nanotechnology of the appearance of quantum effects are discussed.

\section{Classical versus quantum mechanics}

The dynamics of motion of a classical object is characterized by the mass of the object, its position, its velocity (i. e., the speed and direction) or its momentum which is the product of the mass of the object times its velocity. The mass is fixed. The position and velocity or the momentum are continuous variables. For example, the speed of a car can vary continuously from zero to its maximum value. These variables characterizing the object can be measured as accurately as the tools used to make the measurement allow. The evolution of the position and velocity defines the trajectory of the car.

To describe transfers of energy not accompanied by transfers of matter, such as occurs when sound and light (or other electromagnetic radiations) move through space, classical physics employs a picture of wave propagation. The theoretical description is based on solving differential equations with boundary conditions depending upon the specific situation under consideration. For example, vibrations which are confined in space 
generate waves with definite frequencies. Thus, sound waves generated by vibration of a guitar string are periodic and depend on the nature of the string and its length. The string does not generate all sound frequencies (white noise) but just those satisfying the boundary conditions. If the vibration is not confined, as it is the case for waves propagating in free space, all frequencies are possible.

\section{Wave-particle duality}

At the macroscopic level, where classical physics applies, a particle always behaves as a particle and a wave always as a wave. In the microscopic quantum world particles and waves are not as easily separable. Depending upon the conditions, a particle can behave as a wave and a wave as a particle. For example, while, at the classical level light is described by wave equations, in the "photoelectric" and "Compton" effects, light behaves as a particle, the photon. This "wave-particle duality" has many practical implications. The photoelectric effect, ejection of electrons by collisions with photons, is exploited in photovoltaic cells, to transform sunlight into electricity. The Compton effect, scattering of photons by electrons, is used in imaging detectors for medical applications. Electrons or neutrons, which behave in many cases as particles, exhibit wave behavior evidenced by diffraction patterns analogous to those observed in the scattering of light and other electromagnetic radiations. Electron and neutron scattering are used to view small details of small objects and to determine the underlying structures of materials. These are just a few examples of the harnessing of quantum effects in devices that we use routinely. This behavior is schematically depicted in figures 12 and 13 .

The experimental observation of wave-particle duality led naturally to a description of microscopic matter in which it was postulated that each particle had associated with it a wave which governed its motion. The corresponding wavelength of this wave is known as the de Broglie wavelength.

\section{Determinism versus a probabilistic approach}

As indicated by the discussion so far, classical mechanics is a deterministic theory. If we know the initial conditions of the object (mass, position and speed) and the forces acting upon it (force field), it is possible to calculate 


\section{Classical effect}

\section{Quantum effect}
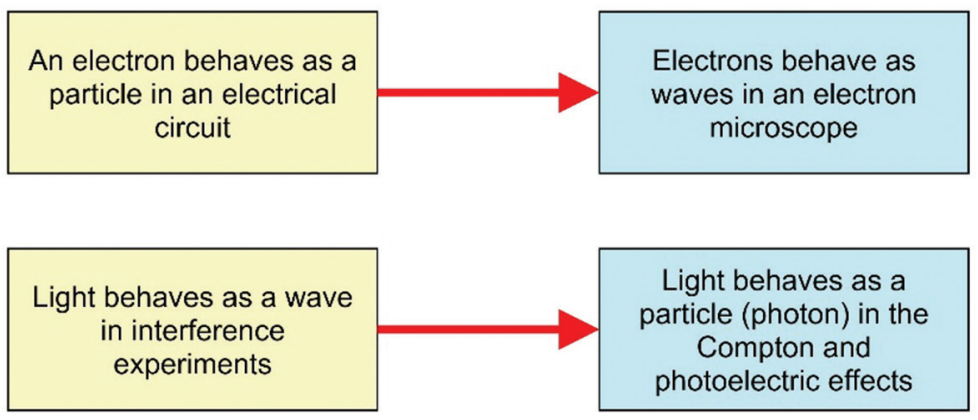

\section{Wave-particle duality}

Figure 12. Quantum mechanics accounts for the fact that a particle can behave as a wave or a wave as a particle according to the boundary conditions placed upon a system. This is illustrated for an electron and light (photons) in this figure.

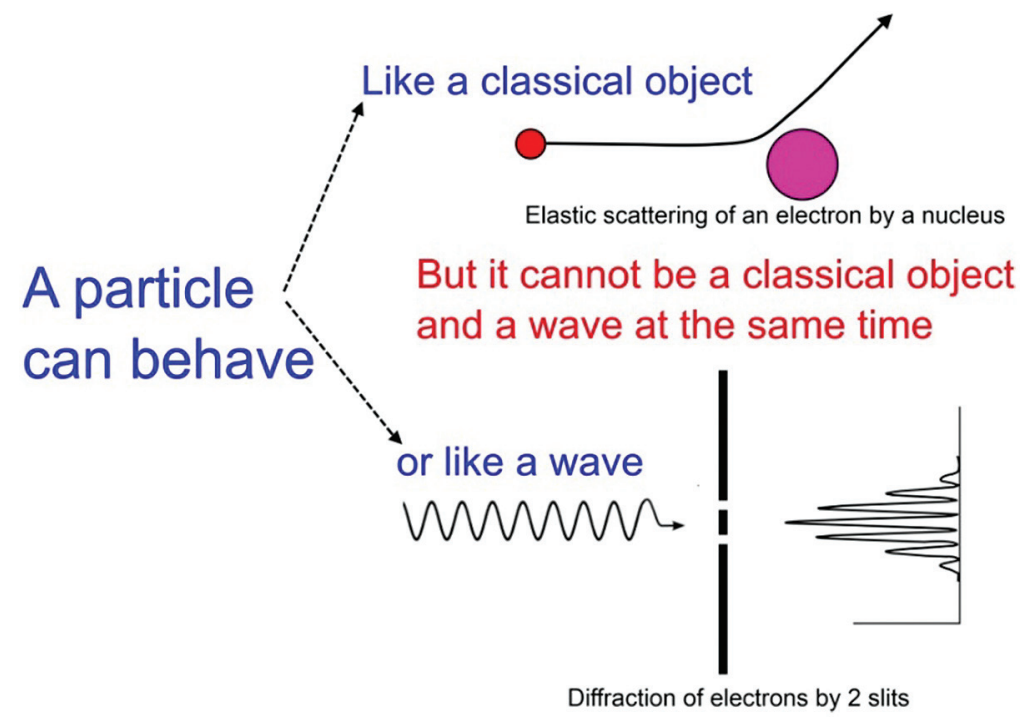

Figure 13. An electron can behave like a classical object or like a wave depending on the conditions, but it can never be both of them at the same time. This illustrates the wave-particle duality. 
the position and the velocity at any later time. The object follows a well-defined trajectory. It has been amply demonstrated experimentally that classical mechanics does not provide an appropriate description of microscopic entities such as atoms, molecules, electrons and nuclei. Instead, these particles manifest wave-particle duality which incorporates features of both particle and wave behaviors. Thus, classical mechanics is not adequate to treat microscopic entities. For these, Quantum Mechanics is the proper theory to apply.

In quantum mechanics the notion of trajectory does not exist anymore. The behavior of the quantum object cannot be characterized completely by its position and momentum, but rather requires a knowledge of its associated wave function. All of the information accessible for the system is contained within this wave function. The wave function is a solution to a wave equation, a differential operator equation which controls the motion of the particle. An operator equation is one in which some specified mathematical operation, e. g., multiplication, division, differentiation...., is performed on a given mathematical function. For each physical quantity that we can measure, there is an associated operator. The theory requires that such operators be "Hermitian" 2 . Figure 14 gives a schematic comparison of some aspects of classical and quantum mechanics.

\section{Measurement}

If we measure the speed of a car with radar waves, we can determine that speed with an error depending on the accuracy of the measuring device used. There is, in principle, no limit to the accuracy, except that of the device we employ. Quantum mechanics differs from classical mechanics in that a measurement is not always deterministic but probabilistic. In quantum mechanics, the result of a measurement can only be a value allowed by the wave function of the operator associated to the observable that we measure. Such an allowed value is called an "eigenvalue". If the system is initially in an "eigenstate", a state allowed by this operator, then the results will be deterministic: Our measurement will produce that particular eigenvalue. A second measurement of the same variable performed on the system will again give the same result.

If the system is initially not in an eigenstate corresponding to one particular eigenvalue of the observable, each of the possible eigenvalues corresponding to allowed states of the system can be, in principle, measured with a certain probability. The same measurement performed on a set of 


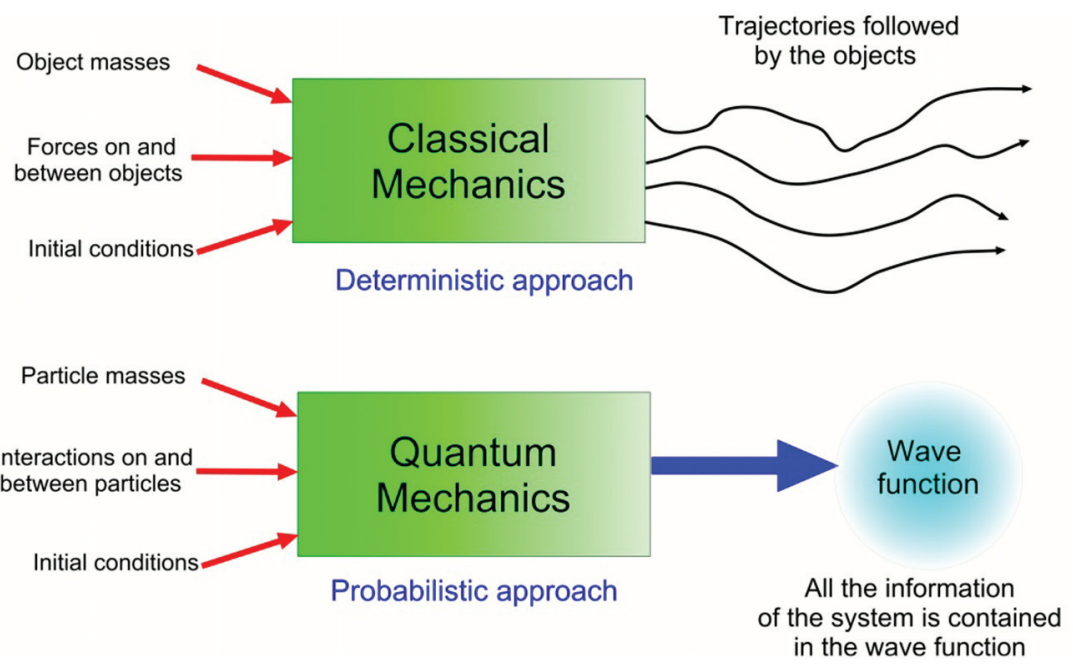

Figure 14. Comparison between classical and quantum mechanics.

such systems will give different results. Figure 15 summarizes this situation schematically.

\section{Quantization}

At the macroscopic level the position and the momentum of a particle are both continuous variables. The kinetic energy of a particle is also a continuous variable. The situation for particles exhibiting wave behavior is different. We have already recognized that confined waves, such as those produced by plucking a guitar string, generate one frequency and its harmonics, and not a white noise associated with continuous frequencies. This means that only certain discrete energy states of the system are allowed. This discretization of the possible energies comes from the boundary conditions which confine the string of the guitar on two sides. More generally stated, spatial boundary conditions lead to energy quantization.

As a result, particles confined (by external forces) to small regions of space cannot have continuous distributions in energy but can only have certain discrete energies. Further, it is interesting to note that, unlike the classical case, the lowest energy state of a quantum system cannot be at zero energy. Even in the lowest energy "ground" state, the position of the particle fluctuates. The non-zero value of the energy is called the "zero point energy". 


\section{Quantum system: illustration of a measurement}

Measurement of the variable $\mathbf{A}=>$ operator $\hat{\mathbf{A}}$
Eigenvalues of $\hat{\mathbf{A}}: \mathbf{a}_{1}, \mathrm{a}_{2}, \mathrm{a}_{3}, \ldots$
Eigenvectors (wave function) of $\hat{\mathbf{A}}: \Psi_{\mathrm{a} 1}, \Psi_{\mathrm{a} 2}, \Psi_{\mathrm{a} 3}, \ldots$

\begin{tabular}{|c|c|c|}
\hline $\begin{array}{c}\text { The system is initially } \\
\text { in state } \Psi_{\mathrm{a} 3}\end{array}$ & $\underset{\substack{\text { Measurement } \\
\text { of } A}}{\longrightarrow} a_{3}$ & $\underset{\substack{\text { Measurement } \\
\text { of } A}}{\longrightarrow} a_{3}$ \\
\hline
\end{tabular}

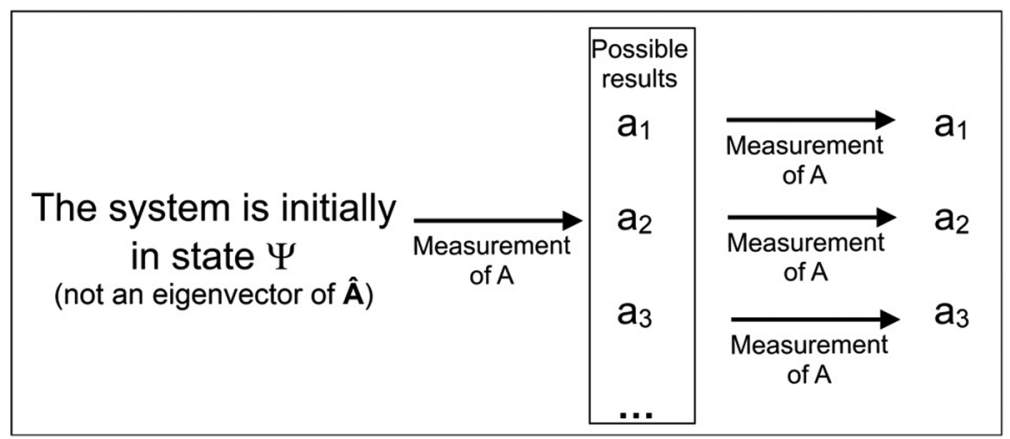

Figure 15. Illustration of results of a measurement on a quantum system. The variable (observable $A$ ) is measured and $\hat{A}$ is the associated operator. If the wave function is in an eigenstate of $\hat{A}$, for example $\psi_{\text {aз }}$, one measures a value $a_{3}$. A second measurement will give the same result. If the system is not in an eigenstate of $\hat{A}$, all the eigenvalues are possible, but with different probabilities.

The simple schematic picture in Figure 16 illustrates these concepts. The external forces confining the particle are represented by a parabola (harmonic oscillator potential). In the classical case shown on the left, the particle can be at any distance above the bottom of this parabola and have any energy up to the maximum energy $E$ indicated by the dashed lines. On the right, in the quantum case, only certain discrete energy levels of the system are allowed. These are indicated by horizontal solid lines. In this illustration, only the lower three states are allowed to the system. The energy spacing between the states will increase if the region in which the particle is confined decreases.

\section{Heisenberg uncertainty principle}

In principle, it is always possible to make a measurement on a classical system without notably perturbing it. Measuring the speed of a car with radar waves does not materially modify the trajectory of the car. Thus, it is possible to measure the position and the momentum (or velocity) 


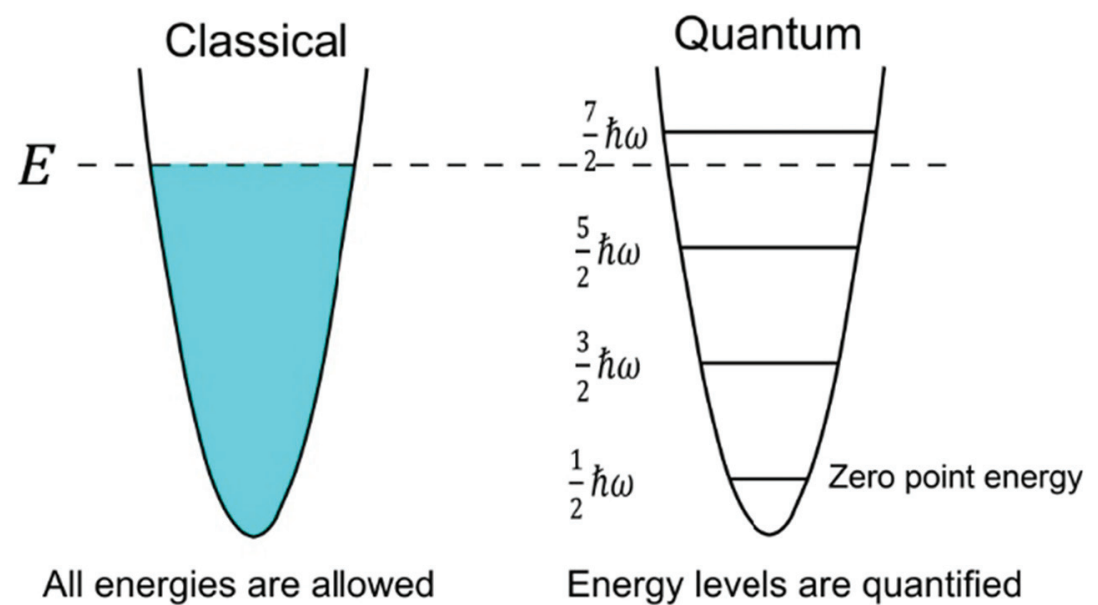

Figure 16. Particle confined by external forces. For the classical case on the left, all the energies from the bottom up to $\mathrm{E}$ are allowed (colored in the figure). For a quantum system only discrete energy values are allowed. Note that in the quantum case the lowest energy is greater than zero.

simultaneously. The accuracy of the measurement depends on the accuracy of the measuring instrument.

At the microscopic level the situation is different. In quantum mechanics, an operator is associated with each variable which can be measured and the result of a measurement is necessarily an "eigenvalue" of the operator. In contrast to the classical case, an accurate simultaneous measurement of two variables (having two different operators) is not possible unless the system is in an "eigenstate" common for both operators. This is related to the "commutivity" or "non-commutivity" of the two operators ${ }^{4}$.

If the two operators do not commute ${ }^{5}$, it is impossible to measure them simultaneously with all the accuracy we wish. This is true of position and momentum which are non-commuting variables. If, for a particle, $\Delta \mathrm{X}$ and $\Delta \mathrm{P}_{\mathrm{x}}$ are, respectively, the uncertainties in the position and momentum along the $\mathrm{x}$ axis, then the "Heisenberg uncertainty principle" may be stated as:

$$
\Delta \mathrm{X} \times \Delta \mathrm{P}_{\mathrm{x}} \geqslant \hbar
$$

Where $\hbar$ is the Planck constant divided by $2 \pi(\hbar=h / 2 \pi)$. It is possible to measure either the position or the momentum as accurately as we want but not both simultaneously. The accuracies achievable are determined by the equation above. A small uncertainty in one of these variables leads to a large uncertainty in the other. 
Figure 17 illustrates this correlation of uncertainties in the $\mathrm{x}$ and $\mathrm{p}_{\mathrm{x}}$ determinations, reflecting the Heisenberg uncertainty principle. The colored rectangles indicate the accuracy limits resulting from the non-commutivity. This same limitation also explains the zero point energy indicated in figure 16. Since the system is confined in a limited portion of space there will be an associated uncertainty in the momentum, and, as a consequence, on the energy. Neither the momentum or the energy can be zero. Associated with this zero-point energy is a zero-point motion. The particle cannot be at rest.

\section{Quantum numbers}

A system is completely defined by the knowledge of its wave function. In the case where the energy levels are discrete, the wave function often depends on indices which characterize a particular energy state of the system. We can designate the wave function of the system, $\psi_{n}$. Here $n$ is an integer number which can be o (the lowest energy or "ground" state) or a positive integer. If $n=1$, the system is in the first excited state, $n=2$, the second excited state, etc. Then, each state can be identified by the appropriate integer, which is known as a quantum number. The energy of the state will depend upon this quantum number. For a system with $N$ degrees of freedom there will be $N$ quantum numbers. (The number of degrees of freedom is the number of parameters of a system which can vary independently.) A classical system with $N$ degrees of freedom requires $N$ position coordinates. For a point particle having no internal degrees of freedom this number is equal to 3 (the three coordinates locating the position). Similarly, a quantum system with $N$ degrees of freedom has $N$ quantum numbers to describe it completely. For example, a point particle inside a cubic box has 3 degrees of freedom and the energy levels depend on 3 quantum numbers.

\section{Spin - an intrinsic property}

A particle can also have intrinsic degrees of freedom which do not depend on spatial position. One such intrinsic degree of freedom for particles such as electrons, protons, neutrons, nuclei etc. is known as angular momentum or spin. Sometimes the spin of a particle is represented as a rotation of the particle around an axis passing through its center. The spin is then a vector. This classical view helps to imagine what a spin can be. Spin is also quantized in quantum systems. Usually quantum angular momenta are measured in 


\section{Heisenberg uncertainty principle}
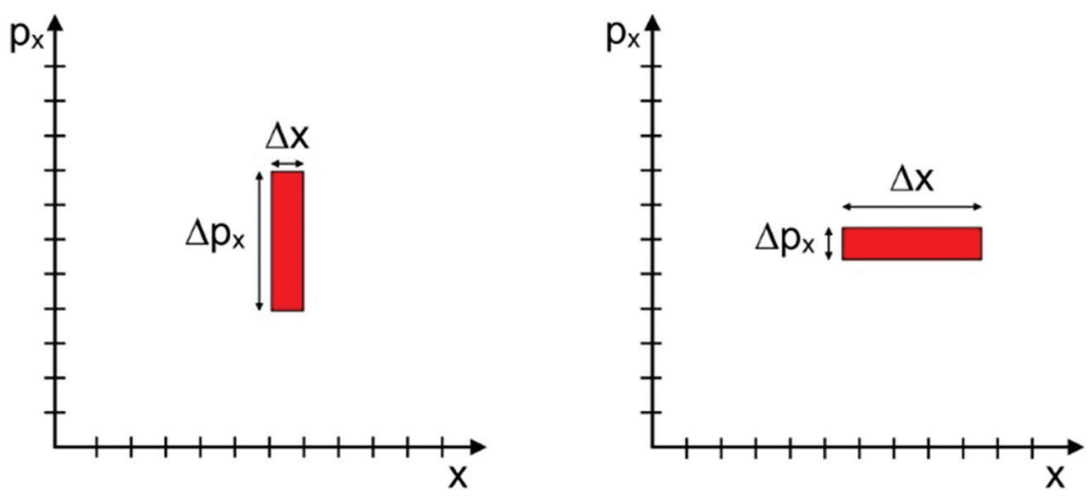

Figure 17. Illustration of the Heisenberg uncertainty principle for the position $(x)$ and the momentum (px) of a particle. These two quantities cannot be measured simultaneously. The better one of these variables is known, the greater is the uncertainty on the other.

$\hbar$ units. Electrons or protons, for example, have a spin $S=1 / 2 \hbar$ and we say that the spin of an electron or a proton is $s=1 / 2$. Quantum mechanics also says that the projection of this $1 / 2 \hbar$ vector on a given axis can take only two values: $+1 / 2 \hbar$ and $-1 / 2 \hbar$, often referred to as up or down. For larger spins, whether half-integer or integer, more projections (actually $2 \mathrm{~s}+1$ values) are possible.

All of the quantum particles found in nature have a spin. This spin can be either an integral spin $(0,1,2 \ldots)$, or a half-integral spin $(1 / 2,3 / 2,5 / 2 \ldots)$. Particles with integral spins are called bosons and particles with half-integral spins are called fermions.

\section{Fermions and bosons}

At the microscopic level, identical particles are indistinguishable. There is no way to differentiate between two electrons, for example. This is in severe contrast to our experience with macroscopic objects such as billiard balls which are, or can be made to be, discernible, e. $g$., by painting them different colors.

Symmetry plays a major role in nature and in quantum mechanics. The application of quantum mechanics to a set of identical particles requires that the total wave function is either totally symmetric with respect to the exchange of any two identical particles, i. e., the sign of the wave function 
does not change when the particles are exchanged, or totally antisymmetric with respect to the exchange of two identical particles, i.e., the sign of the wave function changes when the particles are exchanged. This symmetry requirement is intimately connected to the intrinsic spin of the particles. For bosons, the wave function is symmetric while for fermions it is antisymmetric. Bosons are said to obey Bose-Einstein statistics and fermions to obey Fermi-Dirac statistics.

One of the consequences of this is that, while it is possible to put as many identical bosons as we want into a given quantum state as in the right part of figure 18, completely identical fermions cannot occupy the same quantum state (a quantum state is one identified by all of the pertinent quantum numbers). In the left part in figure 18 we see that 2 fermions of identical spin $1 / 2$ can be placed in a single energy level. This comes from the fact that there are two possibilities for the spin projection, $\mathrm{s}_{\mathrm{z}}= \pm 1 / 2$. The two particles with these different spin projections are not completely identical. However, given the limitation of two spin projections, a third particle of spin $1 / 2$ cannot be placed in the same energy level. In the absence of an external magnetic field the spin of a particle is randomly oriented and the energies for different spin orientations are the same. The application of an external magnetic field results in a splitting of the energy levels as the spins will be oriented only in certain allowed directions relative to the magnetic field.

\section{Quantum tunneling}

Classical energy waves, e.g. electromagnetic waves or sound waves, can tunnel through a potential barrier and an attenuated wave can reach the other side (consider for example the attenuation of a sound wave passing through an acoustic tile). This situation is illustrated in the top of figure 19. There, a classical wave incident from the left impinges on the barrier. In the forbidden region an evanescent wave is formed with a decaying intensity and a wave of diminished amplitude emerges from the other side of the barrier.

In contrast, if a classical particle is incident on such a barrier and its kinetic energy is below the maximum energy of the barrier, it cannot pass through the barrier. To do so requires an energy at least equal to the barrier energy. A tennis ball does not tunnel through a concrete wall because it behaves as a classical object. Classical wave and particle behaviors are different. 


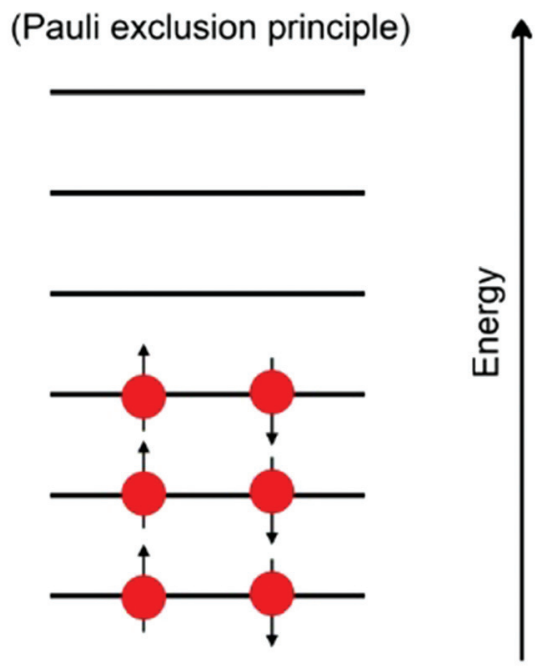

Fermions

\section{Fermion of spin $1 / 2$}

(Bose condensation)

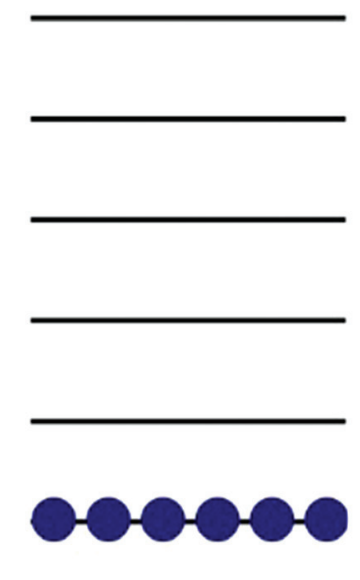

\section{Bosons}

Boson of spin 0

Figure 18. There are two families of particles at the quantum level: fermions and bosons. Completely identical fermions cannot be in the same quantum state. In the case of $s=1 / 2$ particles illustrated here, two different projections of the spin are allowed so two otherwise identical fermions can occupy a single energy level. In contrast, the number of identical bosons in the same quantum state is not restricted.

In a quantum mechanical treatment, wave-particle duality means that in certain conditions a particle can behave as a wave and exhibit a similar tunneling behavior. That is, there is a certain probability that the particle will pass through the barrier and be found on the other side. This is illustrated in the bottom part in figure 19. This behavior is indeed observed for particles such as electrons, protons, neutrons, nuclei, etc. This is precisely the phenomenon used in the scanning tunneling microscope to see and move atoms on a surface.

As it is schematically illustrated in figure 20 , the probability that a particle is able to tunnel through the barrier depends on the relationship between the particle energy and the height of the barrier. It is easier to tunnel with energies close to the top of the barrier than with smaller kinetic energies. The width of the barrier is also important. The larger the width, the smaller will be the tunneling probability. 

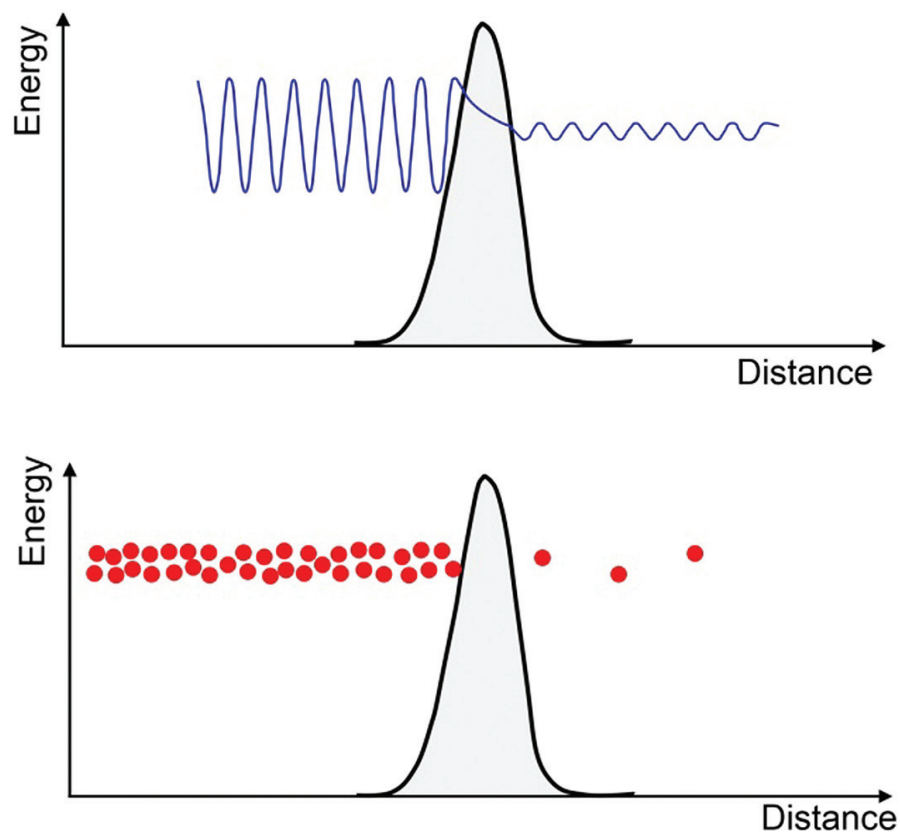

\section{Quantum tunneling through a barrier}

Figure 19. Schematic illustration of quantum tunneling. Top: the wave function decays exponentially inside the barrier and has a lower amplitude after passing through the potential barrier. Bottom: a small fraction of particles striking the barrier tunnel through the barrier.

\section{Summary}

A given system has a behavior which is governed by the laws of physics. By looking at different observables of a system, we can characterize the system. Observables are measureable variables that describe the properties of the system. Observables might be energies, positions, angular momenta, etc. Classical physics (mechanics, hydrodynamics, optics...) describes our macroscopic world well. Classical physics allows us to precisely calculate the trajectories of planets, rockets, vehicles, etc. The microscopic world is very different from the classical world. To adequately describe this microscopic world requires that we apply the concepts of quantum mechanics. This is not because the underlying scientific laws are different. It is rather because the boundary conditions have changed and the relative importance of certain exigencies of the scientific laws can become more or less important with respect to each other depending on the size of the piece of matter we are dealing with. Some effects, which are negligible at the 


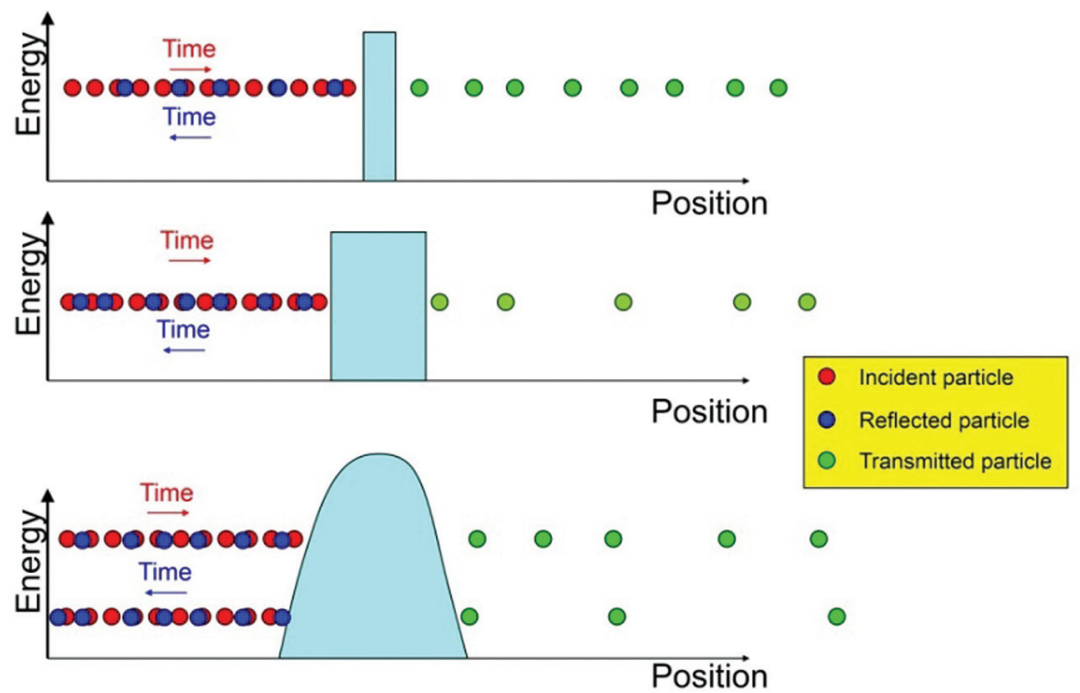

Figure 20. This figure illustrates, in a very simplified and schematic way, the fact that the probability to tunnel through a potential barrier decreases as the difference between the particle energy and the energy at the top of the barrier or the width of the barrier increase. In the figure red particles represent the incident flux on the barrier. Part of this incident flux tunnels through the barrier (green particles) while the remaining part bounces back (blue particles).

macroscale level, can become dominant at the microscale level. Although quantum mechanics is known to provide the foundation for accurately describing microscopic systems and predicting their behavior, a number of the concepts introduced in this chapter can be disconcerting when first encountered. 


\section{The mesoscopic world}

\section{The forces of nature}

Nature is governed by four fundamental interactions:

- The gravitational interaction which operates between objects having mass

- The electromagnetic interaction which operates between objects with electrical charge

- The strong interaction which holds nuclei together

- The weak interaction which governs beta radioactivity and other weak decays

These interactions operate over different ranges and differ greatly in strength. The main properties of these interactions are recalled in figure 21.

The gravitational interaction and electromagnetic interactions have an infinite range while the nuclear and weak interactions are of short or ultra-short range, respectively. In modern theories, the interactions between particles are considered to take place through an exchange of virtual particles (the mediators of the interaction) called gauge bosons. The mediator of the electromagnetic interaction is the photon. The strong interaction needs 8 mediators, known as gluons. In the theory, the elementary particles, the quarks, interact through gluon exchange in the same way as electric charges interact by virtual photon exchange. Three gauge bosons, the $\mathrm{W}^{+}, \mathrm{W}^{-}$and $\mathrm{Z}^{\circ}$ are the mediators of the weak interaction. For gravitation the search for the mediator, called the graviton, is still underway. In February of 2016, scientists working at the The Laser Interferometer Gravitational-Wave Observatory (LIGO) with detectors located in Hanford and in Livingston, Lousiana reported the first experimental observation of gravitational waves. This is a first step towards a possible isolation of the graviton.

\section{The role of size}

Nanoscience and nanotechnology deal with objects intermediate in size between the macroscopic world and the microscopic world. Because of the small dimensions involved, the behaviors of nanoscale objects having dimensions in the range of 1-100 $\mathrm{nm}$ can be very different from the behaviors 


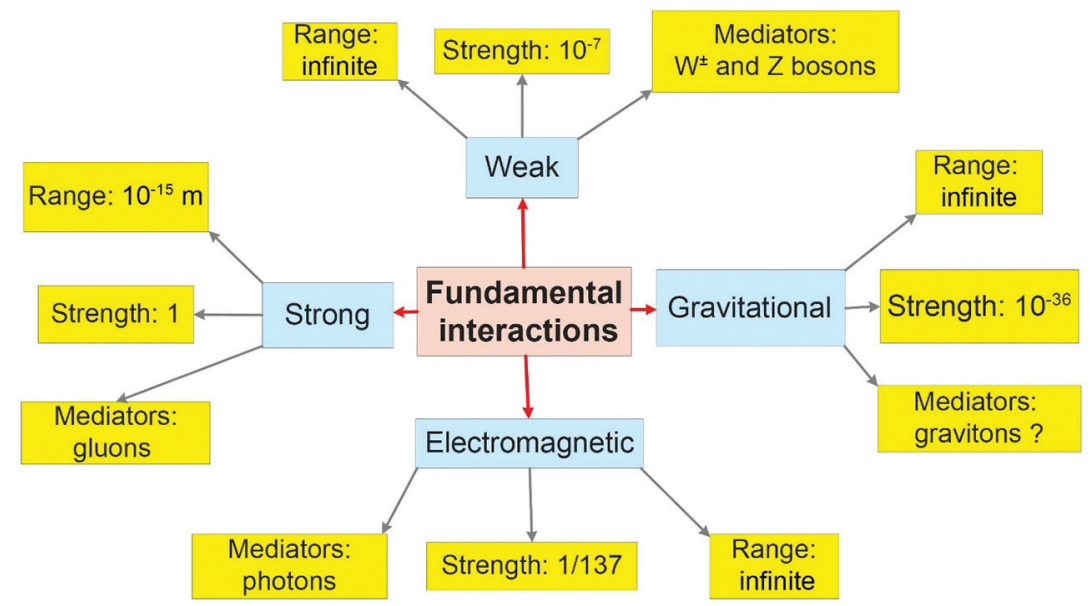

Figure 21. Main properties of the four fundamental interactions. Strengths are on a relative scale.

we have become accustomed to in the macroscopic world. Such objects can exhibit quantum behaviors.

As the size of a system decreases, the relative importance of various forces will change. Also, as the system reaches the nanoscale domain, there can be a transition from classical behavior to quantum behavior. This transition regime is typically referred to as the mesoscopic world. The transition is usually smooth and takes place over some range of sizes. This range can be wide or narrow, depending on the observable under consideration. We will call this region the quantum wall since it separates a region of sizes where classical mechanics is applicable from a region where quantum mechanics governs the physics of the observable. This is illustrated schematically in figure 22.

In this chapter we consider the role of diminishing size on various features of particular importance to nanoscale objects.

\section{Surface versus volume}

A collection of objects of small size generally has a higher surface-to-volume ratio than macroscopic objects containing the same amount of matter. If we consider, for example, a cube with a side of $1 \mathrm{~cm}$, the volume of the cube is $1 \mathrm{~cm}^{3}$ and the surface area is $6 \mathrm{~cm}^{2}$. If we cut it into nanocubes according to the scheme in figure 23, each nanocube has a volume of $1 \mathrm{~nm}^{3}$ and a surface of $6 \mathrm{~nm}^{2}$ or $6 \times 10^{-24} \mathrm{~cm}^{2}$. Since there are a lot of nanocubes, i. e. $10^{21}$, the total 


\section{Quantum wall}

Observable 1

Observable 2

Observable 3

Observable 4

Observable 5

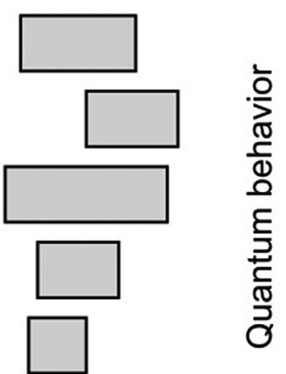

을

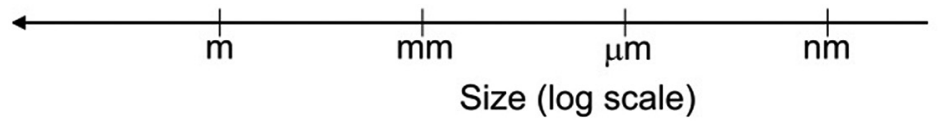

Figure 22. As the size of a system shrinks, the behavior of an observable (a variable that we can measure) can change from classical to quantum. The transition region where this happens is the quantum wall.
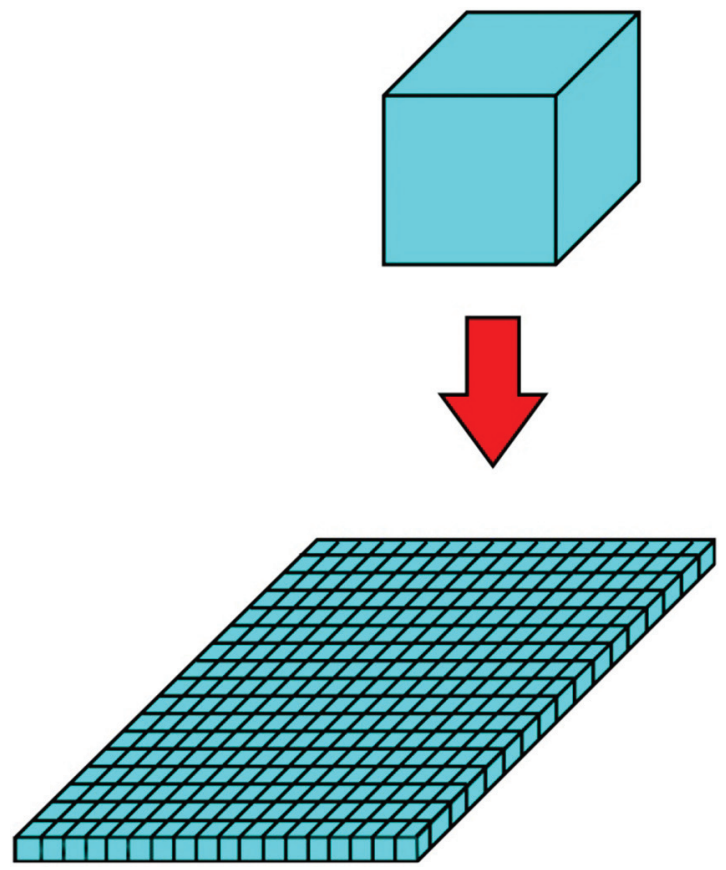

Figure 23. Dividing a cube into tiny little cubes increases substantially the surface area. See text. 
area of this set of nanocubes is $6, \mathrm{ooom}^{2}$. However the total volume of the system is the same $\left(\mathrm{cm}^{3}\right)$. The ratio of surface to volume has increased by a factor of $10^{11}$.

An increase of surface with respect to volume increases the reactivity of a material because the interface with the external environment or with other materials is larger. For example, while iron reacts with oxygen and rusts, it is difficult to burn a lump of iron. However, if this lump is ground into a small powder, or stretched into a fine thread, it burns easily. The key point to remember is that, at the nanoscale, surface effects can become dominant over volume effects.

\section{Surface tension}

For liquids, increases of surface to volume ratios can result in greatly increasing the importance of the surface tension. Surface tension finds its origin in short range intermolecular forces. Such forces are discussed in more detail in section 8 , below. For a given liquid, molecules on the surface typically have stronger attractions to molecules in the bulk of the liquid than to external molecules. In response to this difference, the liquid will tend to form drops in order to minimize the surface energy. Surface tension explains the stability of soap bubbles, the coalescence of two touching drops into a single one and why dew does not spread on flower petals.

For large volumes of liquid, surface tension is not as important as other forces which are operating. This explains why the water contained in a glass assumes the shape of the glass. Nevertheless, the surface tension exists. This explains why needles can float and small insects can easily walk on that surface. It is easy to understand that, due to surface tension, nanosized objects will have difficulties penetrating into a liquid.

\section{Nanofluids}

Unless quantum effects appear, the laws of fluid dynamics are the same, whatever the size of an object moving in a fluid. However, the fluid reacts differently depending on the size of the object. The nature of fluid flow is characterized by a parameter known as the Reynolds number. The Reynolds number is a dimensionless quantity which is the ratio of the inertial forces to the viscous forces. In the case of a body entering a fluid, the 
Reynolds number is proportional to the characteristic speed of the body and to its size and inversely proportional to the viscosity coefficient of the fluid. Two fluids with the same Reynolds number exhibit the same flow pattern. In the case of low Reynolds numbers, the flow is laminar, i. e., the liquid flows in parallel layers whereas for large Reynolds numbers it is turbulent. Viscous forces become increasingly important on the nanoscale. At the macroscopic scale, we know that turbulence provides a way to mix two different fluids together. At the nanoscale, turbulence is reduced because of the viscous forces and it is very difficult to mix two liquids using this phenomenon.

\section{Heat transfer}

Another issue at small sizes is the transfer of heat energy. Three main phenomena govern heat transfer at the macroscopic scale: conduction, convection and radiation. For nanostructures of very small size, heat transfer mechanisms are different. The classical Fourier law of heat conduction states that the rate of heat transfer through a material is proportional to the gradient in the temperature and to the area, at right angles to that gradient, through which the heat flows. On the nanoscale, this law breaks down because the mean free path of the particles becomes larger than the size of the nanostructure. The mechanism is closer to radiation than to conduction. Furthermore, at the nanoscale, because of the wave-particle duality, wave effects become important. In the mesoscopic world, where sizes intermediate between the microscopic and the macroscopic world are involved, heat transfer becomes a complicated process depending on several parameters such as the mean free path of the particles, their thermal wave length, their phase coherence, etc.

\section{Chemical bonding}

Most materials are composed of atoms of different elements bound to each other to form chemical compounds. The bonds between atoms in these compounds find their origin in the electromagnetic interaction. They vary in strength depending on their nature. In chemical compounds, the bonds between atoms range between covalent bonds, in which electron pairs are shared between two atoms, to ionic bonds, in which oppositely charged ionized atoms attract each other. The bonds are strong and not 
easy to break. Carbon-hydrogen covalent bonds have an energy of the order of $400 \mathrm{~kJ} / \mathrm{mole}$ of molecules. Ionic bonds are of comparable strengths to covalent bonds. In ionic crystals, ions located the lattice sites are bound to each other by electrostatic forces. Each ion feels the long range force from all the other ions. The net bonding is significantly larger than that between a single ion pair.

\section{Intermolecular forces}

There are also attractive interactions between atoms and molecules which are much weaker than covalent or ionic bonds, but play a major role on the properties of materials. These are generally classified as Van der Waals interactions or Van der Waals forces. As indicated in section 4, the Van der Waals forces are responsible for such phenomena as the coalescence of drops and formation of bubbles.

\section{Van der Waals forces}

The Van der Waals forces occur between atoms or molecules. They can also be present between a molecules and crystalline surfaces. They are of three types:

1. The Keesom force coming from the interaction between polar entities having permanent electric dipole moments ${ }^{5}$.

2. The Debye force occurring between entities with permanent dipole moments and those with induced dipole moments.

3. The London force coming from the interaction between entities both of which have induced dipole moments.

These 3 forces are attractive and the strengths of these forces depend on the relative distance $r$ between the two entities. The change is proportional to $1 / r^{7}$. The potential energy varies like $1 / r^{6}$. For comparison the electrostatic interaction between two charged particles varies like $1 / r^{2}$.

The Keesom force is a dipole-dipole interaction exerted between two polar molecules. The force tends to align the dipoles to increase the attraction between the species and minimize the potential energy.

The Debye force is an interaction between the permanent dipole of a polar molecule and an dipole induced in an initially non-polar molecule. 


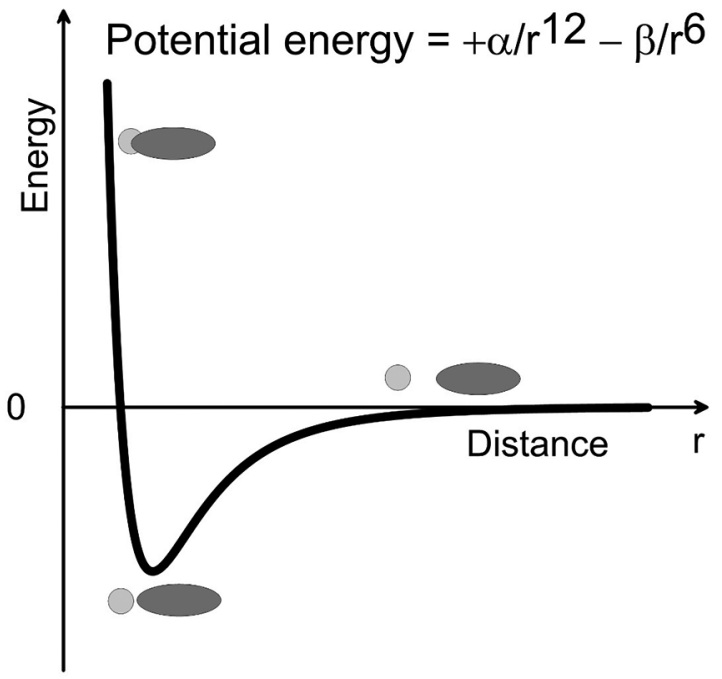

Figure 24. Schematic illustration of the potential energy curve between atomic and molecular interactions via Van der Waals forces. The repulsive core reflects the Pauli energy coming from the Pauli exclusion principle. The two parameters $\alpha$ and $\beta$ in the expression of the potential energy are determined theoretically or chosen to match experimental data.

The London forces exist between all molecules. It is the only Van de Waals interaction observed with non-polar molecules and is due to internal charge fluctuations in these molecules. In a molecule, moving electrons create an instantaneous but fluctuating dipole moment. The order of magnitude of the London forces is $\approx 2 \mathrm{~kJ} /$ mole. London forces keep the atoms of noble gases close together when they are liquefied and are responsible for the fact that benzene is a liquid at room temperature.

At very short distances the interaction between atoms and molecules turns from attractive to repulsive reflecting the fact that two identical electrons cannot be in the same quantum state ,i.e., the total wave function should be antisymmetric (the Pauli exclusion principle). This constraint on the total wave function gives rise to a short range repulsive force varying like $1 / r^{13}$ (the potential energy empirically varies like $\left.1 / r^{12}\right)$. This repulsive force is called the Pauli or Born force.

Figure 24 shows a schematic view of the potential energy of an atom and a molecule as a function of the distance $r$. This kind of potential is called a Lennard-Jones potential. As the molecules come close together the potential energy decreases because of Van der Waals interactions and then increases strongly due to the repulsive Pauli or Born interaction. 
At the nanoscale, the Van der Waals forces are much more important than gravitational forces.

\section{Hydrogen bonds}

Because of its unusual strength compared to that of other attractive Van der Waals forces, the hydrogen bond can be of particular importance. The hydrogen bond is an attractive electromagnetic interaction between a hydrogen atom already bonded to another atom in a polar molecule and a lone pair of electrons on a strongly electronegative element such as fluorine, oxygen or nitrogen. The strongest hydrogen bonds involve hydrogen-fluorine interactions. Hydrogen bonds can occur between atoms belonging to different molecules or between two atoms belonging to the same molecule.

The energy of a typical hydrogen bond is $\approx 20 \mathrm{~kJ} / \mathrm{mole}$, about an order of magnitude smaller than a strong covalent bond. The length of the bond is also about twice that of a covalent bond. Though significantly weaker than covalent bonds, hydrogen bonds can be extremely important. They are responsible for the high boiling point of water compared to other non -metallic hydrides ${ }^{6}$ and thus the fact that most of the water on the earth's surface is liquid at typical ambient temperatures. Hydrogen bonding is also the interaction which holds the double helix structure of DNA together.

\section{Semi-classical approaches}

Describing the microscopic world (atoms, molecules, nuclei...) requires us to use quantum mechanics. Quantum systems are characterized by quantum numbers which vary from small values to very large values. The quantum numbers of a system requiring a full quantum mechanical treatment are usually small because they are not too far from the ground state. In contrast, classical systems correspond to situations where the values of the quantum numbers are extremely large. As we noted above, nanotechnology covers a domain where some observables behave as classical and others behave quantum mechanically. This depends upon the nature and size of the system as well as on the external conditions. Fortunately, in many areas of nanotechnology, it is possible to meld the ideas of the classical and quantum approaches into semi-classical approaches which employ a large part of the classical thinking and invoke, ad hoc, a few quantization rules. These semi-classical approaches can then be employed to characterize nanoscale 


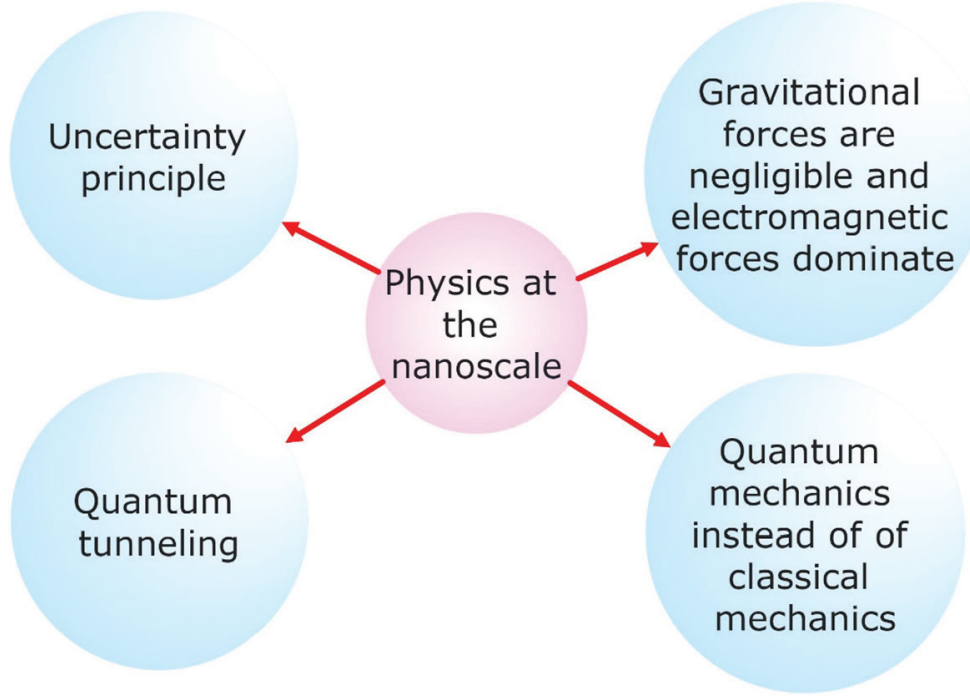

Figure 25. Important concepts manifested at the nanoscale.

phenomena. ${ }^{7}$ To the extent that a semi-classical approach captures the principal features of the phenomenon, it is useful because its application is easier than solving the full quantum problem exactly. Furthermore, because the concepts are more aligned with those of our macroscopic world, it facilitates grasping and communicating the essential features of the phenomenon.

\section{Summary}

As the dimensions of a system decrease and reach the nanometer range, new phenomena can be observed. Some of these can be understood as reflecting the natural size evolution of macroscopic behavior tempered by the relative change in importance of the forces operating on the system. In particular this is the case for surface effects which become more important than bulk effects. But there are also quantum phenomena which appear because the classical description is no longer adequate at these dimensions. In figure 25, we emphasize some concepts which play an important role in the physical and chemical behaviors of nanoscale objects. 


\section{Nanomaterials and nanostructures}

A large part of the progress of human society is based on the development of materials. In the past metals have played a tremendous role in this respect. Progress in manufacturing techniques and the creation of new alloys have fostered advances in agriculture, transportation, warfare, cooking, etc. Various periods of rapid development in human history are identified by these advances: the age of copper, which occurred after the stone age, the bronze age, the age of iron. Today we are entering a new period corresponding to the age of nanomaterials. Creating new nanomaterials and understanding their properties will have far-reaching effects, Eiji Kobayashi, from Panasonic, says: "those who control materials control technology". This statement is certainly true for nanomaterials and their growing sociological and economic importance should not be underestimated.

\section{Classifying nanomaterials}

According to The International Organization for Standardization a "nanomaterial" is a material with any external dimensions in the nanoscale range or having internal structure or surface structure in the nanoscale range. This size range is typically considered to be from approximately $1 \mathrm{~nm}$ to $100 \mathrm{~nm}$. Nanomaterials are of particular interest because the unique properties, electrical, magnetic, optical etc., which can emerge on this size scale have wide application in a variety of fields.

It is possible to classify nanomaterials differently depending on which properties one focuses on, i. e., external or internal.

If attention is focused towards the external characteristics of a material, it is possible to classify it according to the external dimensions of the object. If only one dimension is smaller than $100 \mathrm{~nm}$, it is considered as a one-dimensional $(\mathrm{1D})$ nanomaterial. Ultra-thin films are an example of $1 \mathrm{D}$ nanomaterials. If 2 dimensions of the system are in the nanometer range, it is a two-dimensional nanomaterial (2D). Finally, if 3 of the dimensions of the system are smaller than $100 \mathrm{~nm}$ the material is a $3 \mathrm{D}$ nano-object. Nanoparticles or quantum dots belong to this category. Figure 26 summarizes some types of nanomaterials which can be produced and tailored to be utilized in applications such as those discussed in the remainder of this book. 


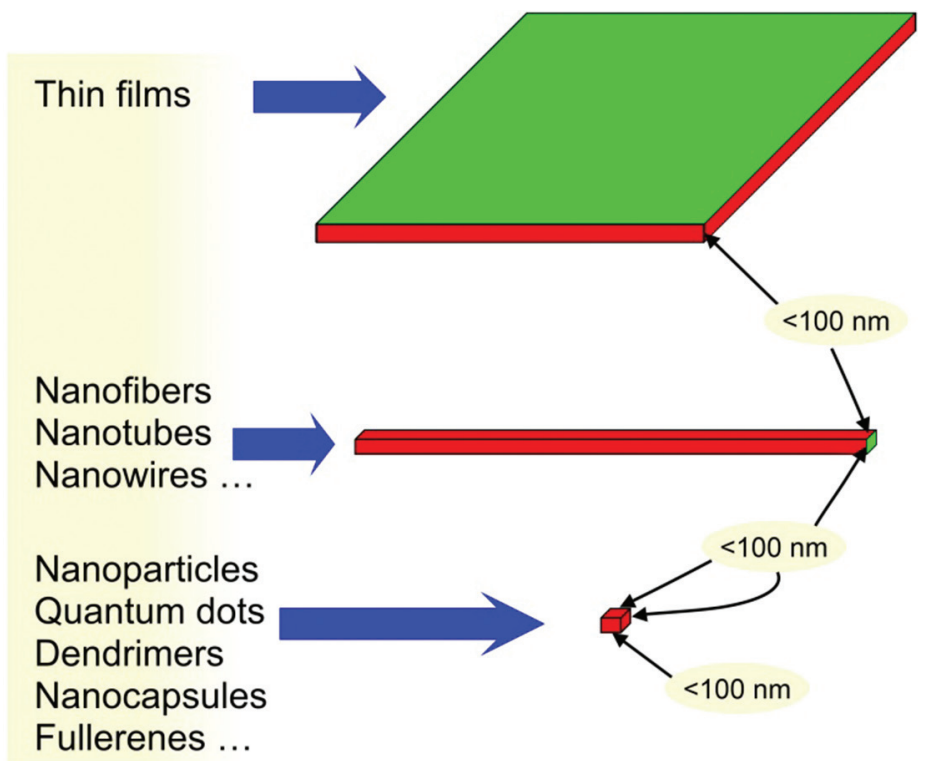

Figure 26. Some nanomaterials of interest.

In this classification, nanomaterials include:

- Materials such as thin films or surface coatings that are nanoscale in one dimension and are extended in the other two dimensions.

- Materials such as nanofibers, nanowires and nanotubes that are nanoscale in two dimensions and extended in the third dimension.

- Materials such as particles or molecules that are nanoscale in all three dimensions.

- Larger nano-crystalline materials composed of nanometer-sized pieces.

Man-made nanomaterials existed before people talked about nanotechnology. One of the examples developed over several decades, is ultra-thin films. The advantage of coating a surface with nanoparticles is that less material is needed to make the film and a better contact is realized. This is illustrated in figure 27 showing a monolayer of particles. Small particles provide a better surface coverage with less material than larger particles.

To show the importance of using ultra thin films in terms of material savings, suppose that we want to deposit a liquid film of one nanometer thickness on the surface Paris city. The question is: how much volume of liquid do we need? The area of Paris city is $105.4 \mathrm{~km}^{2} \approx 10^{26} \mathrm{~nm}^{2}$. A volume of liquid of $10^{26} \mathrm{~nm}^{3}=0.1 \mathrm{~m}^{3}=100$ liters is enough to do that. This simple 


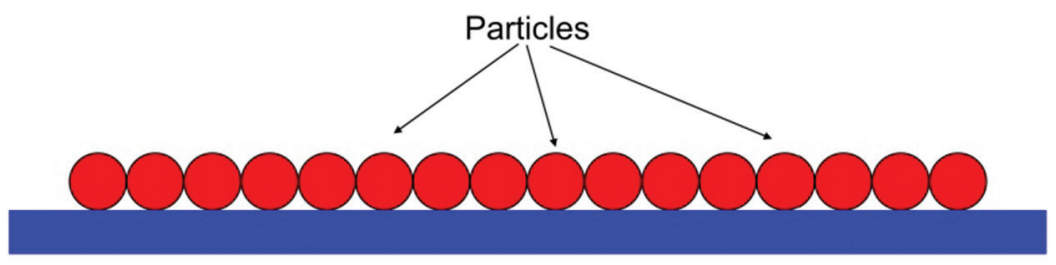

Much smaller particles

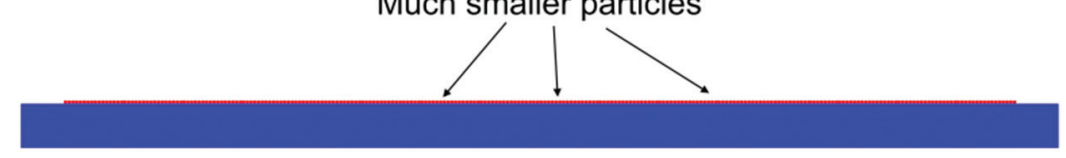

Figure 27. Difference between a monolayer made of particles (full circles) of different sizes coated on a surface.

example shows that the amount of material needed to cover Paris city is pretty small. Using nanosized films instead of microsized films, with the same properties, saves a lot of material and is a good for sustainable development.

Another way to classify nanomaterials is to focus attention on the internal structure of a macroscopic material and especially on the size of the nanostructures which are embedded within it (figure 28). In this classification, a structure has a non-zero dimension if one of its dimension at least is larger than $100 \mathrm{~nm}$. For example, a nanoparticle is a nanostructure with all three dimensions smaller than $100 \mathrm{~nm}$. Therefore, it is considered as a oD (zero dimension) object. A nanowire or a nanofiber has just one dimension larger than $100 \mathrm{~nm}$, consequently it is a $1 \mathrm{D}$ object. An ultra-thin film has two dimensions larger than $100 \mathrm{~nm}$ and is considered as a $2 \mathrm{D}$ nanomaterial. It is also the case of a stack $2 \mathrm{D}$ layers embedded in the bulk material. A ${ }_{3} \mathrm{D}$ material will be just a bulk containing microstructures instead of nanostructures.

\section{Nanostructuration}

Nanostructuring a material is interesting if improved properties are obtained. This can be done either by including specific nanostructures, or by an appropriate process generating nanostructures. Introducing nanostructures enhances the effects that are displayed in figure 29. It creates a large number of interfaces between the nanostructures and the bulk material, generating globally high surfaces in contact. These nanostructures, which are trapped in the bulk, show a size confinement into tiny parts of the bulk. 


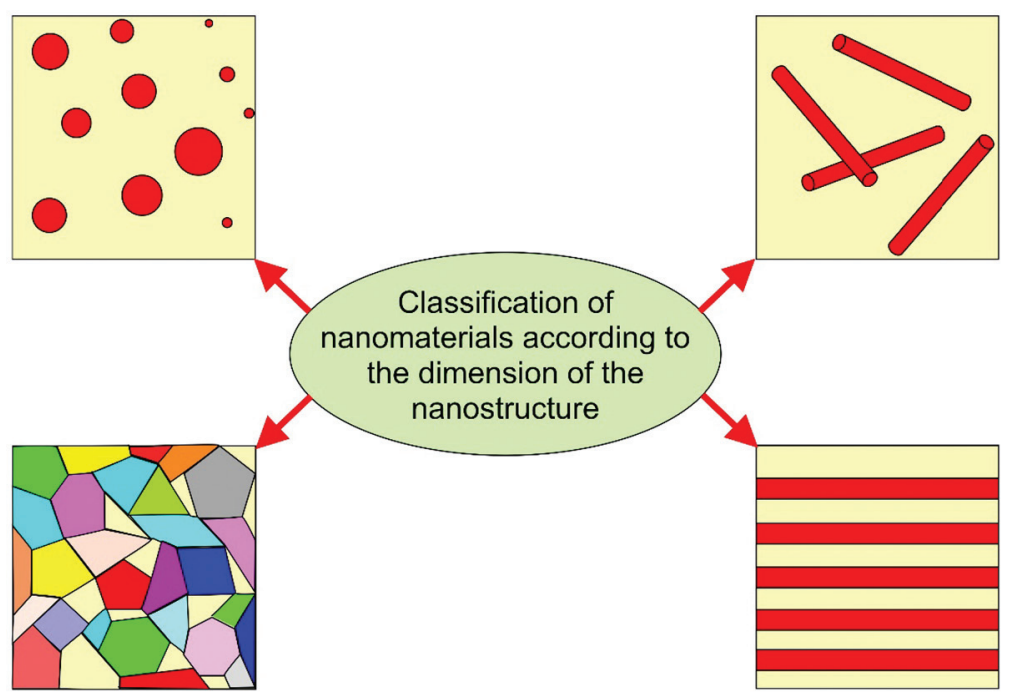

Figure 28. Classification of materials according to the dimensionality of the nanostructures. Illustration is made for $0 D, 1 D, 2 D$ and 3D nanomaterials according to this classification.

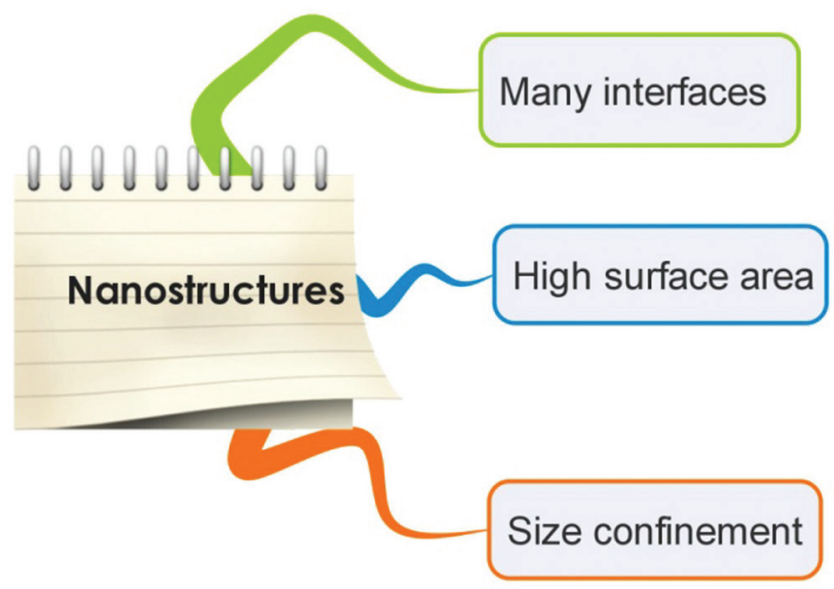

Figure 29. Properties of nanostructures bringing new features in nanostructured materials.

The size and the nature of the nanostructures which are included in a bulk material can induce major changes in the properties of the original material and improve some of the characteristics which are required for a particular application. The nanostructures can introduce specific optical or magnetic properties, for example. The nature of the nanostructure 
and the distribution of the nanostructures in the bulk material will play a key role in determining the new properties. For example, the crystallographic orientation, the chemical composition or the possibility of having out-of-equilibrium nanometer crystallites of gold or sodium chloride can lead to interesting properties of the nanostructured material. Three keypoints need to be investigated in the development of nanomaterials. They are indicated in figure 30.

Dramatic changes can be obtained. For instance, nanoparticles of black carbon included in tires improve several properties such as the ride quality and wear resistance. Adding silica smoke containing nanoparticles in concrete make it less porous and less permeable to water. This new feature is worth for concrete staying in contact with sea water.

Nanoparticles possessing specific properties can also be introduced in the bulk material. There is a great variety of characteristics which are worth to provide to the bulk material such as optical, magnetic, catalytic... properties.

Nanostructures are the building blocks of nanostructured materials. The simplest nanostructures which can be used are nanoparticles but it can be more complicated structures such as fullerene, nanocrystallites, nanotubes...

For example, by dispersing ferromagnetic grains of a size of 5 and 10 nm inside a high-resistivity amorphous film, a nanostructured film of $\mathrm{FeHf}(\mathrm{NO})$ is obtained with a high permeability and resistivity. This nanostructured material is interesting because this material can be integrated close to inductive components and minimizes parasitic capacities at high operating frequencies (up to more than $2 \mathrm{GHz}$ ).

A crystallite is a small region of solid which is a single crystal. Figure 31 schematically shows an arrangement of several crystallites forming a polycrystalline material. Crystallites with a size in the nanometer range are called nanocrystallites. Materials used can be metals or metal oxides. For very small dimensions quantum effects can appear. Metal nanocrystalline materials are often used in heterogeneous catalysis.

An illustration of the structure of a nanocrystalline material is displayed in figure 32. Nanocrystals are embedded into an amorphous matrix.

In this chapter we shall focus our attention on some types of nanomaterials only, and postpone to next chapter the presentation of carbon-based nanostructured materials. 


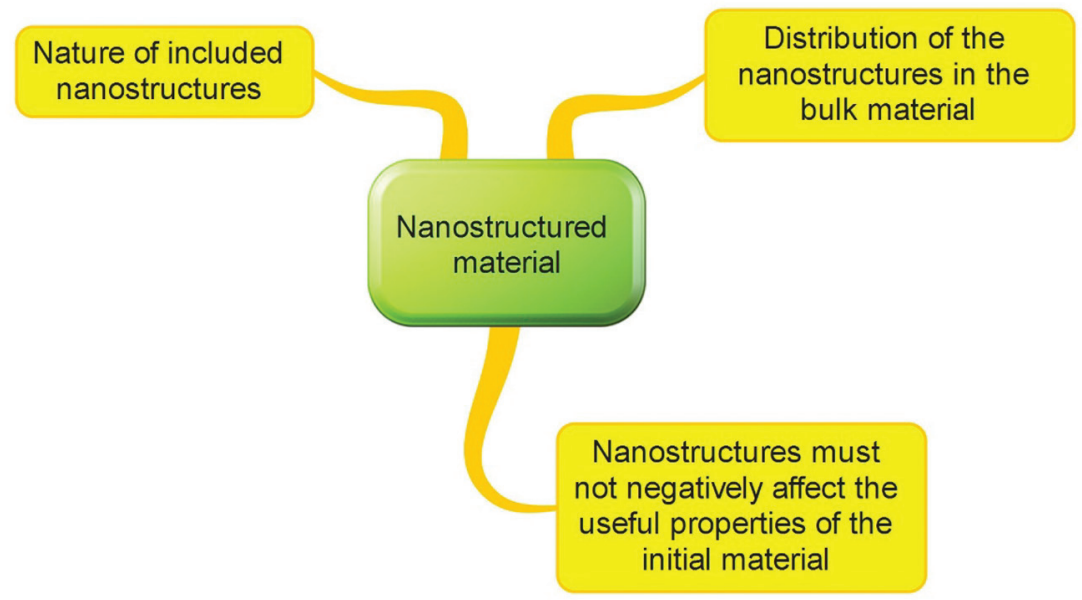

Figure 30. Three keypoints of nanostructured materials. Nanostructures included in materials can give to the nanostructured material new properties that depend on the nature of the nanostructures as well as on their geometrical distribution inside the material. Care should be taken not to negatively affect the initial properties of the material which are useful to the designed application.

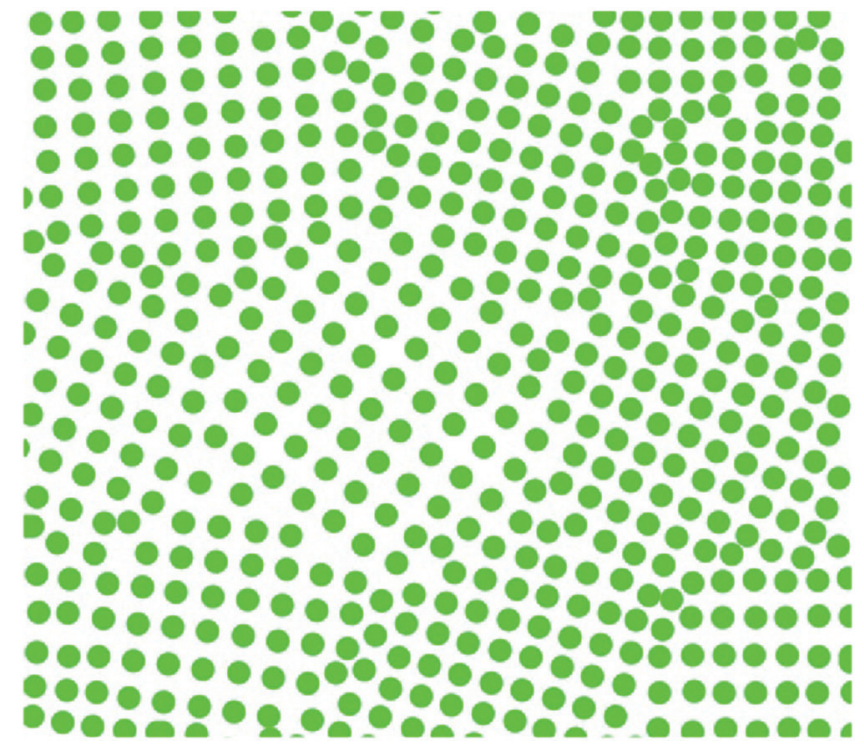

Figure 31. Schematic representation of polycrystalline material consisting of crystallites. Image from Wikimedia Commons (http://commons.wikimedia.org). Author Edward Pleshakov. 


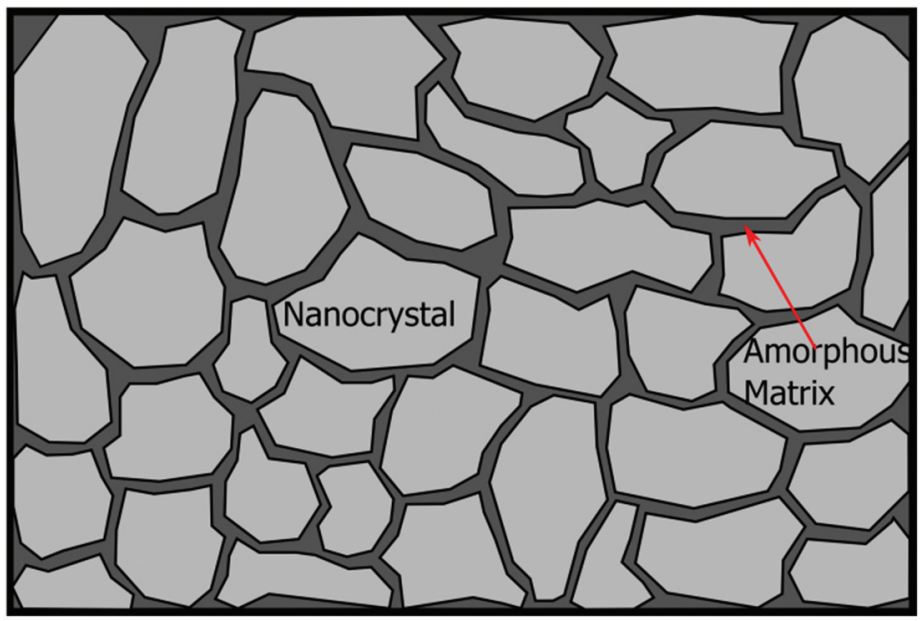

Figure 32. Schematic structure of a nanocomposite material with a nanocrystalline component embedded within an amorphous matrix. Image from Wikimedia Commons (http://commons. wikimedia.org). Author Freundchen.

\section{Dendrimers}

A dendrimer is a macromolecule made up of monomers which are assembled in a tree structure around a central core. It is a highly branched ${ }_{3} \mathrm{D}$ structure with the ability to encapsulate nanoparticles. Figure 33 shows schematically the construction of a $2 \mathrm{D}$ dendrimer by the commonly used divergent method. The dendrimer grows outward from a core molecule containing several reactive sites. The principle illustrated in figure 33 is the following. In the first step, the core molecule reacts with monomers containing one reactive group and two dormant groups. This generates a first-generation dendrimer. The dormant groups are activated and react with monomer molecules to give the second-step dendrimer. The synthesis is repeated until the proper size of the dendrimer is obtained.

The other way to synthesize dendrimers is the convergent method. The end groups are the starting point and the synthesis is inward. A comparison between the two ways of synthesizing dendrimer is shown in figure 34 .

Dendrimer chemistry was introduced in 1978 by F.Vögte and collaborators and the first family of dendrimers was synthesized in 1985 by D.A.Tomalia. Today it is possible to synthesize complex dendrimer structures and control the size, the shape, the surface and interior chemical functionalization. An example of dendrimer is shown in figure 35 . This molecule has 54 ferrocenes attached at the surface. 


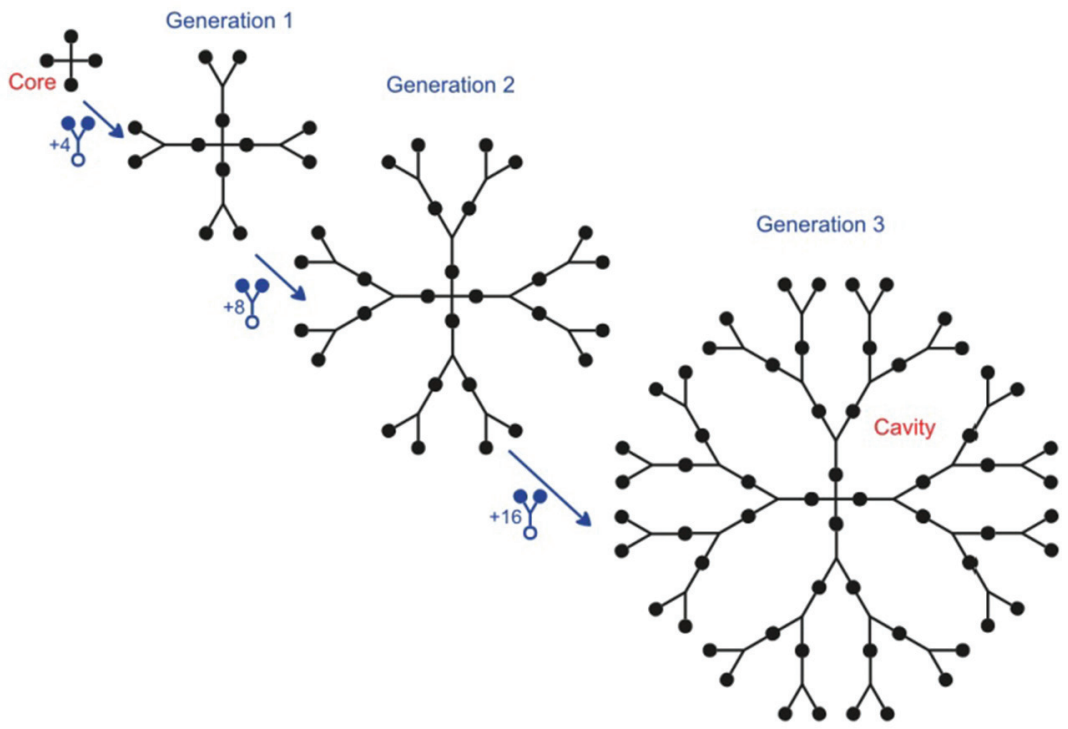

Figure 33. Schematic synthesis of a dendrimer by the divergent method.

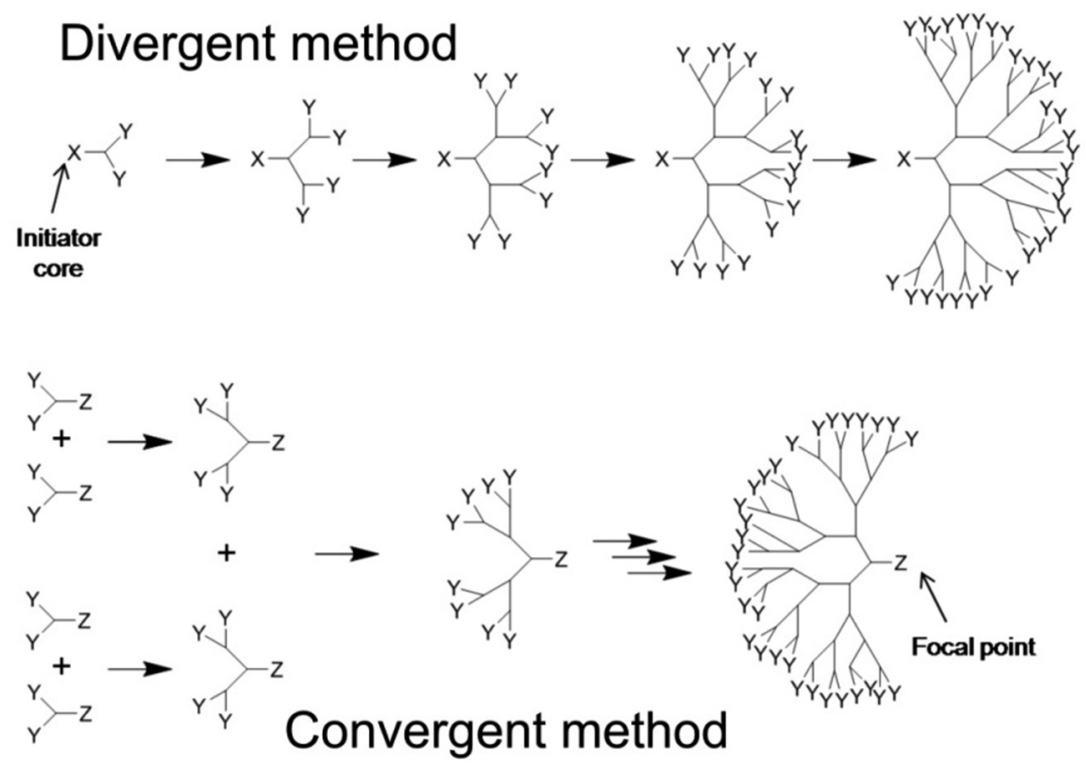

Figure 34. Principle of divergent and convergent method of synthesizing dendrimers. Image from Wikimedia Commons (http://commons.wikimedia.org). Author Chem538w10grp10. 


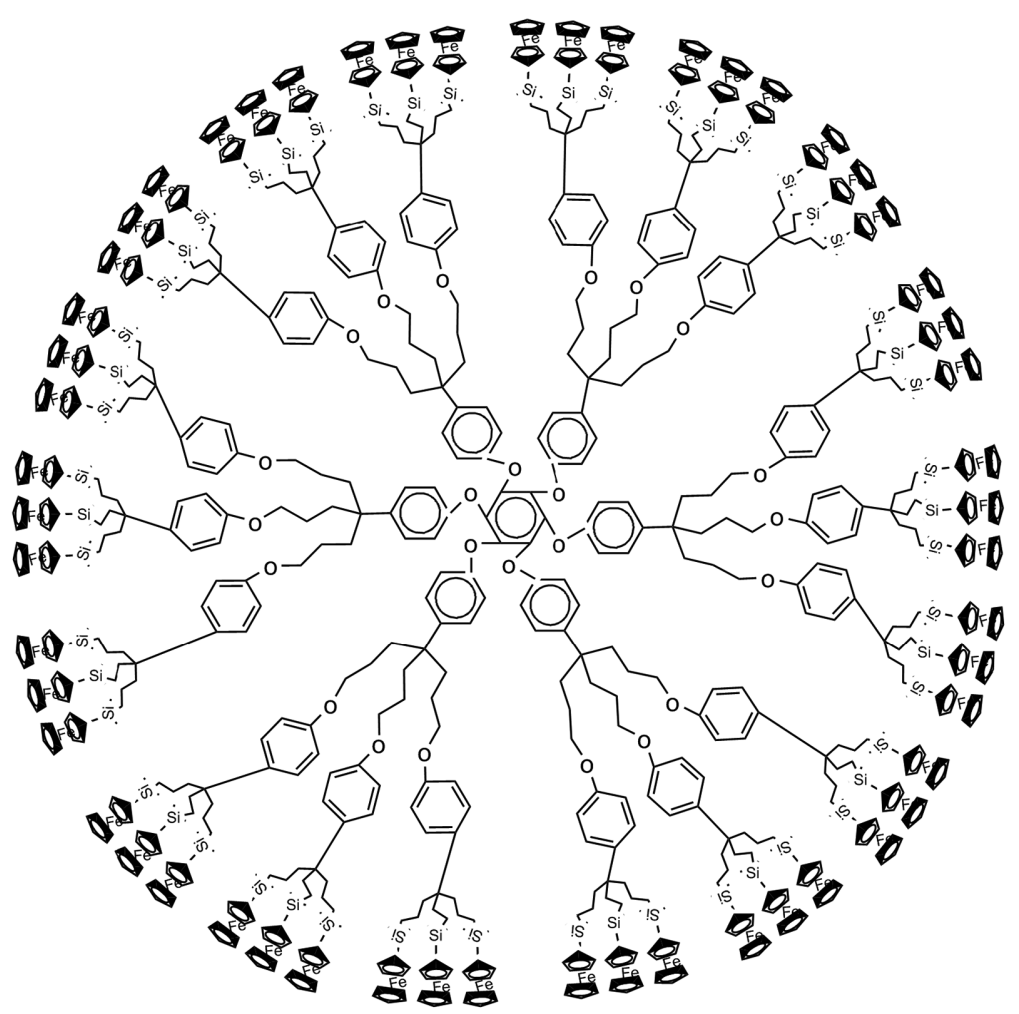

Figure 35. Dendrimer with 54 ferrocenes attached. . Image from Wikimedia Commons (http:// commons.wikimedia.org). Author Skydivermonkey.

Dendrimers can be used to carry drugs for applications such as drug delivery, diagnostics or tumor therapy. Investigations are also carried out to use dendrimers as blood substitutes.

\section{Hybrid organic-inorganic nanomaterials}

Hybrid organic-inorganic nanomaterials are interesting because they have on one side the good physical properties of ceramics and, on the other side, the convenience of organic molecules in terms of reactivity and functionalization.

Among these hybrid materials, the silsesquioxanes have interesting properties. Their stoichiometric formula reads $\mathrm{RSiO}_{3 / 2}$, where $\mathrm{R}$ is an organic radical. Their name derives from the $3 / 2$ oxygen. Sometimes they are also called T-resins ( $\mathrm{T}$ for tri-substitued) because silicon is bonded to 3 oxygens substituants. Their most common geometrical structure is either a ladder 
or a cube. The cubic structure is commonly called the $\mathrm{T}_{8}$-cube. It has the peculiarity that the $\mathrm{O}-\mathrm{Si}-\mathrm{O}$ angle is not $90^{\circ} \mathrm{C}$ although it is often drawn like that. Figure 36 shows an example of a $\mathrm{T}_{8}$ cubic structure of silsesquioxane.

The cage structure shown in figure 36 gives the ability to put small molecules inside. Silsesquioxanes can be functionalized and used as antimicrobial coatings. Using for example quaternary ammonium salts, it is possible to make molecules which can kill bacteria and fungi while being harmless to humans and animals. Silsesquioxanes are also important for heterogeneous silica-supported transition metal catalysts.

\section{Nanocomposites}

Nanocomposite materials cover a wide domain with many applications. A nanocomposite material is a material composed of several phases where at least one of the phase has a dimension in the nanometer range. Nanoparticles included into a matrix are an example of a nanocomposite material. It is also the case of multilayers structures, which are a stack of utra-thin films of different materials. Magnetic multilayers are for example used for storage media.

Inclusion of nanoparticles in a bulk material change its physical properties. It can become stronger, lighter, more ductile... The final properties of a nanocomposite depend on many factors such as the process of manufacturing, the nature of the nanostructure and the arrangement of the nanostructure within the bulk material.

A common example of nanocomposite is a tire. In this object, carbon black nanoparticles have been added in the initial rubber. Their presence improves the strength, tensile properties, the tear and abrasion resistance... However, a too high concentration of nanoparticles in the material lowers these properties.

The concentration of nanoparticles in the initial material plays a key role in the final properties. For example, a few ppm (parts-per-million) of nanoimpureties in aluminum alloys increases substantially the strength and the corrosion resistance of aluminum.

Polymer-clay nanocomposite can improve substantially the properties of a low-cost polymer. For example they are less flammable while keeping their good mechanical properties. Such nanocomposites can be synthesized using sol-gel techniques which are realized in mild conditions. Figure 37 shows the principles of a general synthesis procedure for a nanocomposite hydrogel. Hydrogels are 3-dimensional polymer networks containing a large amount of water. They are usually fragile materials. Nanocomposite hydrogels 


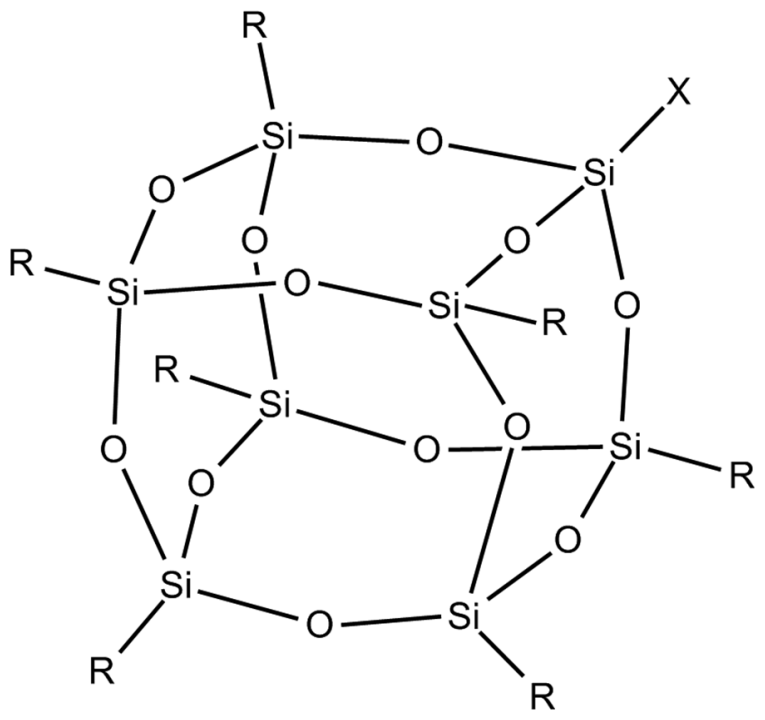

Figure 36. Example of a T8 cubic structure of Silsesquioxane. Image from Wikimedia Commons (http://commons.wikimedia.org). Author Sephirothazule.

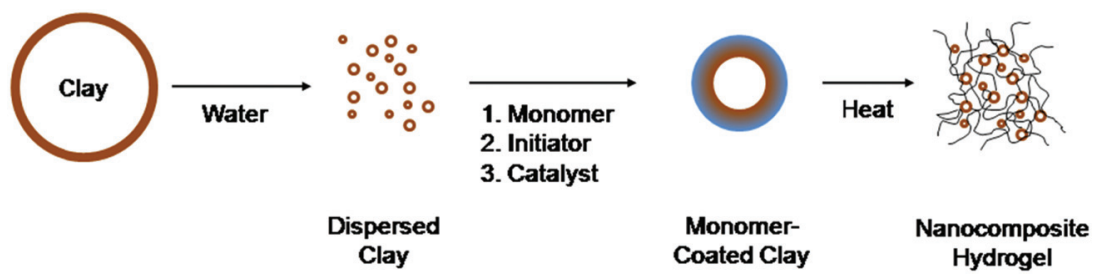

Figure 37. General synthesis procedure for a nanocomposite hydrogel. Image from Wikimedia Commons (http://commons.wikimedia.org). Author Chem538w10grp6.

synthesized with mild chemistry has made a breaktrough in this field. It is now possible to manufacture nanocomposite hydrogels with various shapes and surfaces, with high toughness, with excellent optical properties and with the ability to respond to external stimuli.

\section{Smart materials}

Smart materials are materials which can appropriately respond to stimuli provided by their environment. These materials are usually composite materials containing perception, response and control capabilities of various kinds (figure 38). External stimuli can be of various kinds: light, heat, electricity, radio waves, strain, etc. The materials are able to detect and 


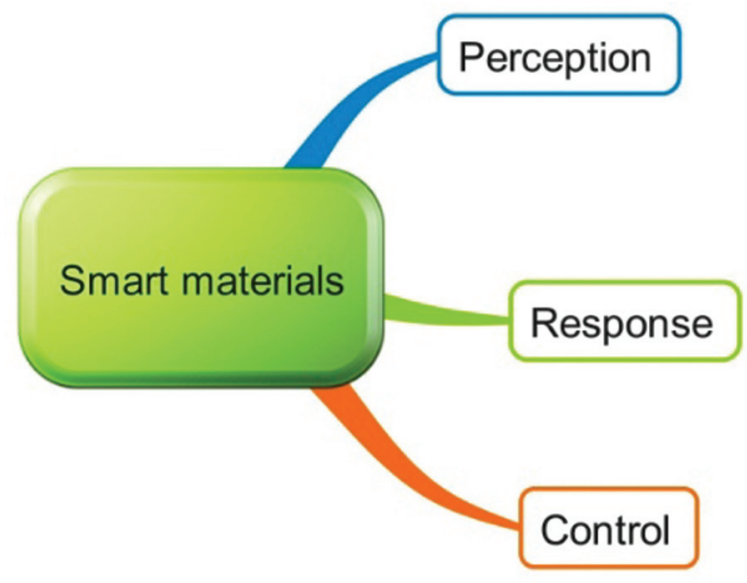

Figure 38. Main properties that a smart material should possess.

identify these stimuli above certain threshold levels and respond to them by choosing among a number of solutions decided by the designer. They should provide these responses within an appropriate time span and at the right level. The process should be reversible. After fulfilling its function, the smart material can be returned to its initial state. Some examples of smart materials are thermoelectric materials capable of converting temperature differences into electricital signals, shape-memory alloys or polymers which can return to their original shapes, and piezoelectric materials sensitive to external pressure changes. Nanotechnology provides new opportunities to fabricate smart materials.

\section{Summary}

Nanomaterials will play increasingly important roles in the future, offering new or improved properties compared to materials which are not nanostructured. Nanotechnology will be applied to build new materials incorporating a number of desired capabilities. Nanotechnology also offers the opportunity to employ less material to accomplish the same goal. This is particularly important for sustainable development because the world may soon be short of some essential raw materials if we continue to use them as we do now. A full exploitation of the potential of nanomaterials demands a dependable understanding of their properties and mechanisms on all dimensional scales. The development of multiscale models, able to predict the properties of a material on all size scales is an important goal in this field. 


\section{Natural nanomaterials}

Materials are made of atoms. They are called natural materials if they belong to the mineral or living world and if no human modification or processing of their structure has been made. Natural materials have properties at the macroscopic level which are often due to their nano or microstructures.

\section{Nanomaterials in nature}

A natural nanomaterial (figure 39) is a material exhibiting properties which reflect its structure at the nanoscale.

There are plenty of examples of natural nanomaterials; some of them are displayed in figure 40 and discussed in the following sections.

\section{Self-cleaning surfaces}

Lotus flower leaves or taro leaves have a self-cleaning property (figures 41 and 42). Water droplets do not wet the surface. This phenomenon comes from superhydrophobicity, i. e, a very high water repellence. The surface of the lotus leaf is rough on the nanometer scale and covered with a waxy material. This material is hydrophobic and the rough surface reduces the area in contact with the leaf. The combination of both effects makes the droplets of water roll right off, taking out dirt that is on the surface. As a consequence, the surface is self-cleaning.

Nanomaterials which mimic this lotus effect have been developed to create surfaces which remain clean and dry, e. g. self cleaning windows or waterproof clothes.

Contrasting materials, surfaces that capture water also exist in nature. The Namib desert beetle uses special nanostructures to capture moisture from fog. A possible application of this phenomenon could be to manufacture surfaces trapping moisture to use as a water source. 


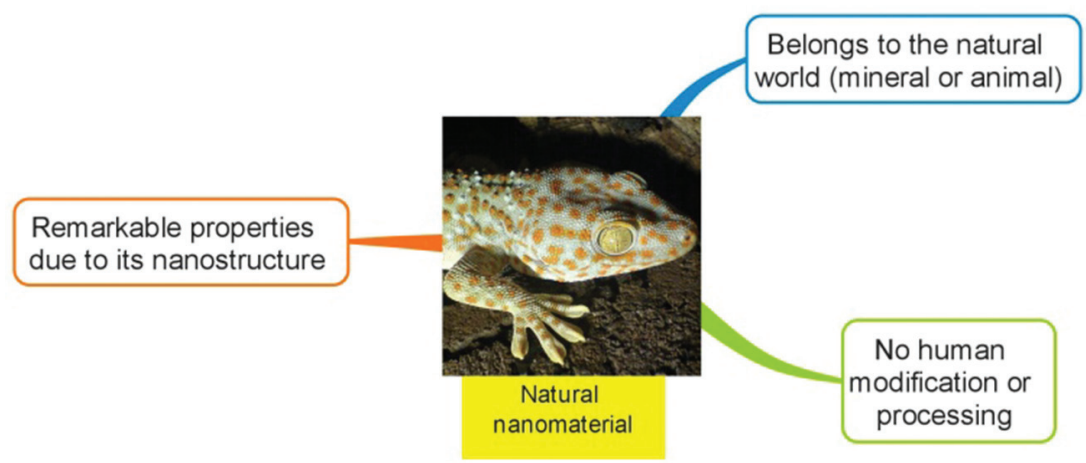

Figure 39. Definition of a natural nanometerial. The image of the Gekko comes from Wikimedia Commons (http://commons.wikimedia.org), author Robert Michniewicz.

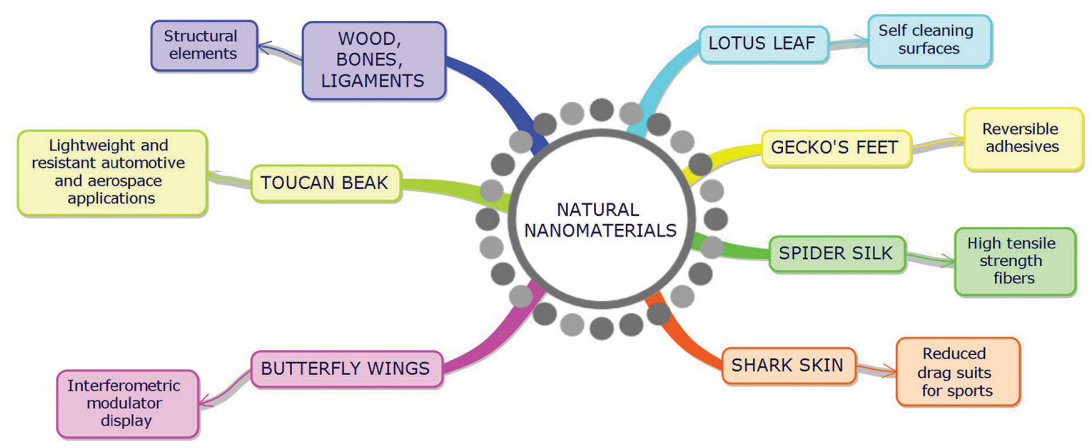

Figure 40. Examples of natural nanomaterials and some biomimetic applications.

\section{Reversible adhesives}

The gecko is a lizard which can cling to any surface at any orientation. It can for example cling and walk on the ceiling with its feet over its head. It can cling on smooth as well as on rough, wet or dirty surfaces. While flies, for example, have similar abilities the gecko is the heaviest animal which can do this.

The weight of an adult Gecko is of the order of $140 \mathrm{~g}$ and, on a vertical wall, it can tolerate about $4 \mathrm{~kg}$ of additional weight without sliding. This astonishing reversible adhesive property is due to millions of setae (tiny bristles and hairs which are typically $100 \mu \mathrm{m}$ long and $5 \mu \mathrm{m}$ in diameter) located on the soles of the gecko's feet. A Gecko foot is shown in figure 43. Each sata ends with hundreds of tiny pads (spatulae, with dimensions of the order of $200 \mathrm{~nm}$ in width and length) helping to cling to a surface (figure 44). Adhesion between the feet and the surface is due to Van der 


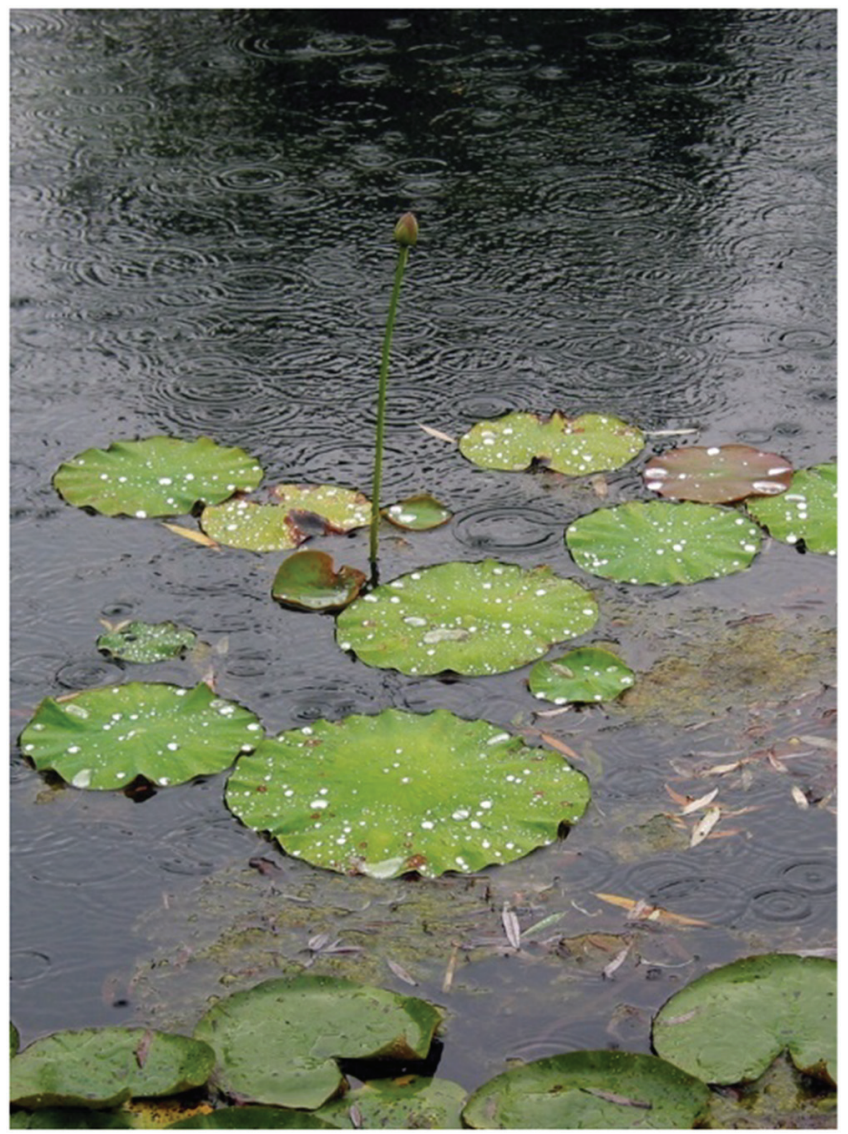

Figure 41. Lotus leaves on pond in rain, showing the so called lotus effect; picture taken in the Chinese Garden at the "Gärten der Welt" (gardens of the world) in Erholungspark Marzahn, Berlin / Germany. Picture from ArchiKat, Wikimedia Commons (http://commons.wikimedia.org).

Waals forces discussed in chapter 3. Since there are a lot of hairs, the total interaction is large and this explains why the adhesion is so strong. The strong adhesive force between the hairs and the surface can be easily relaxed. In other words we have a reversible adhesive. The presence of hairs makes the feet of the Gecko self-cleaning. If dirt-clogged hairs are put on a surface, the dirt particle is left on the surface because there is a smaller contact area between the dirt particle and the satae than between the dirt particle and the surface.

If all the setae on a gecko's feet were active, the gecko could support the weight of two-medium sized persons (a shear force of 1,300 newtons is generated by the $\sim 6.5$ million satae). Actually, the number of active satae 


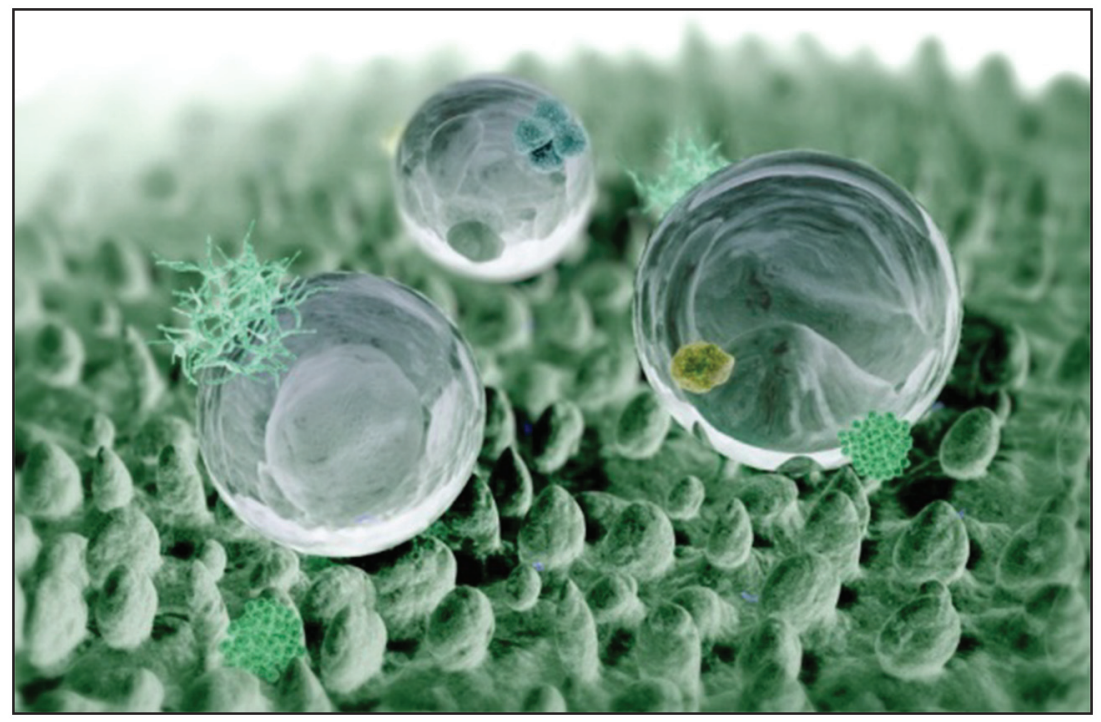

Figure 42. Computer graphics of lotus effect., Picture from Wikimedia Commons (http://commons. wikimedia.org) William Thielicke.

of a gecko is very small, of the order of $3 \%$ but this still creates a very strong force (about 20 newtons).

Several attempts have been made to mimic the Gecko and develop reversible adhesives based on nanoproperties. One goal is surgical band aids that can remain for a long time and which can be removed without pain. The basic idea is to use contact splitting on the nanoscale.This technology was first developed in the US where scientists have used machines usually employed to etch patterns on microchips to create a rubber structure analogous to the gecko's foot. As in the gecko's foot, each hair was covered with thousands of protrusions which are responsible for strong adhesive power. On this nanostructured texture, scientists have added a biodegradable glue giving the ability to the bandage to remain glued when wet. First experiments were made on pigs and rats intestines in 2008 with success. It was found to work better than stitches. For human applications, such band aids can also be impregnated with drugs.

\section{Lightweight materials}

In the aerospace or automotive industries it is important to have strong but light materials. Such materials exist in nature. The beak of a Toucan 


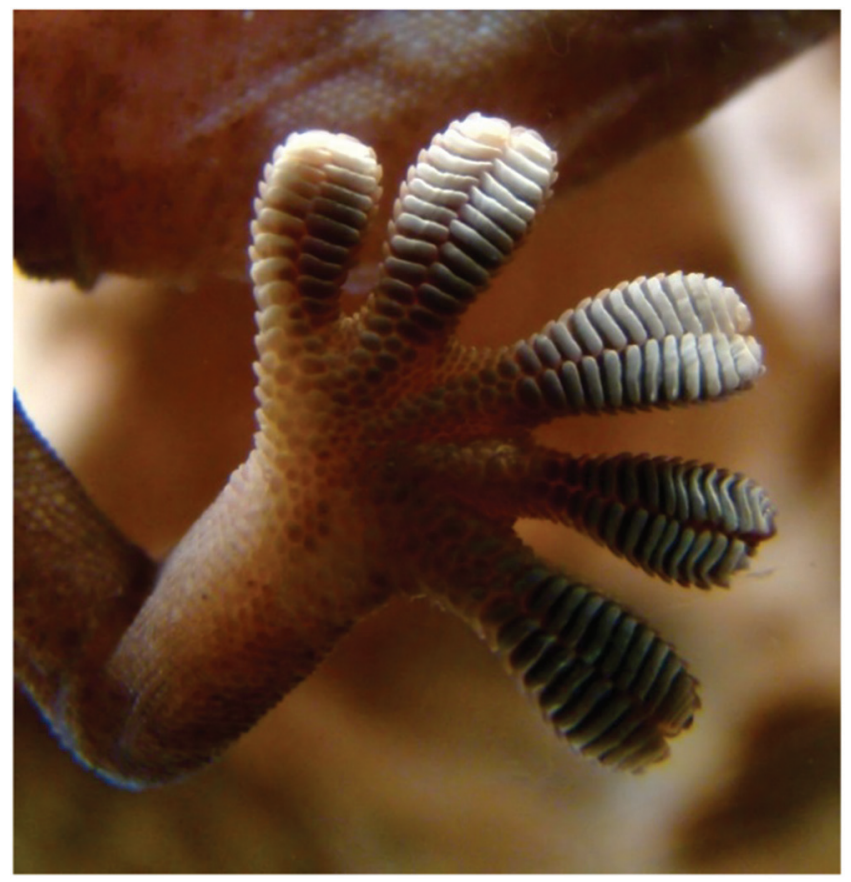

Figure 43. Close-up of the underside of a gecko's foot as it walks on a glass wall. Van der Waals force interactions between the finely divided setae (hairs on the toes) and the glass enables the gecko to stay in place and walk on the seemingly smooth glass. Image from Bjørn Christian Tørrissen, Wikimedia Commons (http://commons.wikimedia.org), and http://bjornfree.com/galleries.html.

(figure 45) is a very strong and light biocomposite. It has hard nanotiles of a protein (keratin which also makes up fingernails, hair and horn) outside and nanosize fibers of bone inside acting as a lightweight supporting foam.

The structure of the toucan beak is interesting because of its high strength to weight ratio and because it is an excellent absorber for high energy impacts. Developing analogous materials for motorist protection in crashes can have many economic and safety implications.

\section{Manipulating light at the nanoscale}

Butterflies (figure 46) manipulate light at the nanoscale thanks to nanometer size structures on their wings. Ridges with dimensions smaller than $1 \mu \mathrm{m}$ have nanostructures allowing for multiple reflection of light leading to beautiful iridescent colors. Similarly constructed artificial structures could be used to develop new fabrics, anti-counterfeit technologies for currency or dye-free paints. 


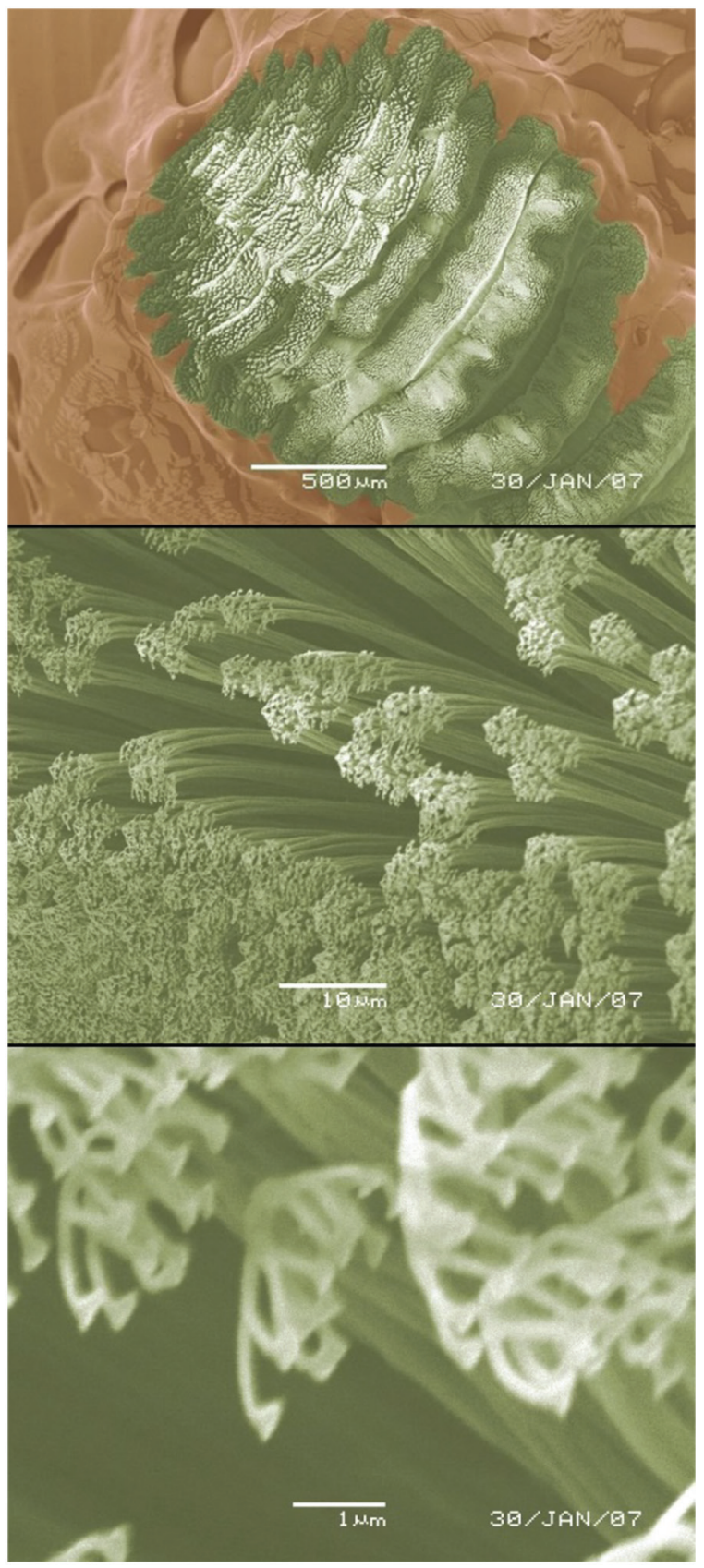

Figure 44. Foot of a Gecko viewed by a scanning electron microscope with increasing magnifications. As magnification increases, we see more and more clearly the tiny hairs. Image from Oskar Gellerbrant, Wikimedia Commons (http://commons.wikimedia.org). 


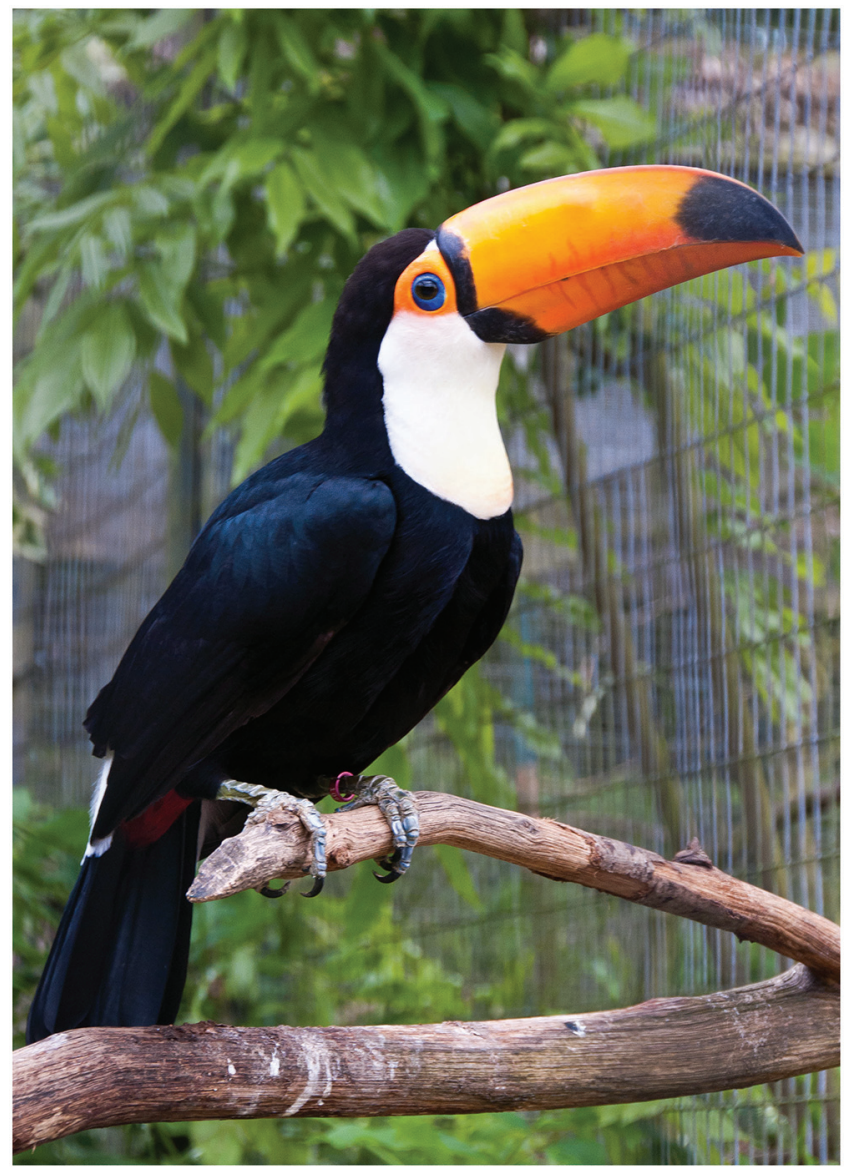

Figure 45. Toucan.

Interferometric modulator displays (IMOD) employ a bio-inspired non -emissive technology where colors are created by controlling how light interferes with itself. The manipulation of light is done with micro-electromechanical systems. One of the advantages is the extremely low energy consumption of displays based on this technology.

\section{Fibers stronger than steel}

The spider web (figure 47) is a very strong natural material. For the same weight, the silk of spider web has a strength about 5 times greater than that of steel. Furthermore, it is elastic and can be stretch up to 10 times its initial length. Spider silk consists of protein molecules produced by 


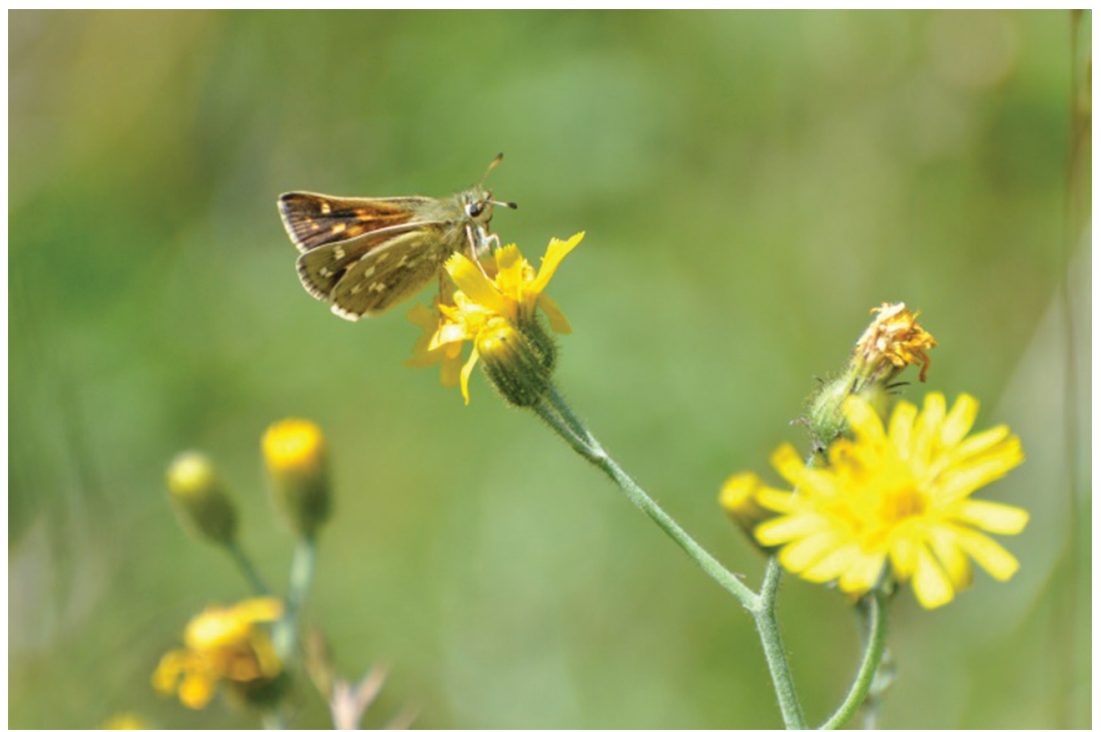

Figure 46. Wings of butterflies have a nanostructure responsible for special optical properties.

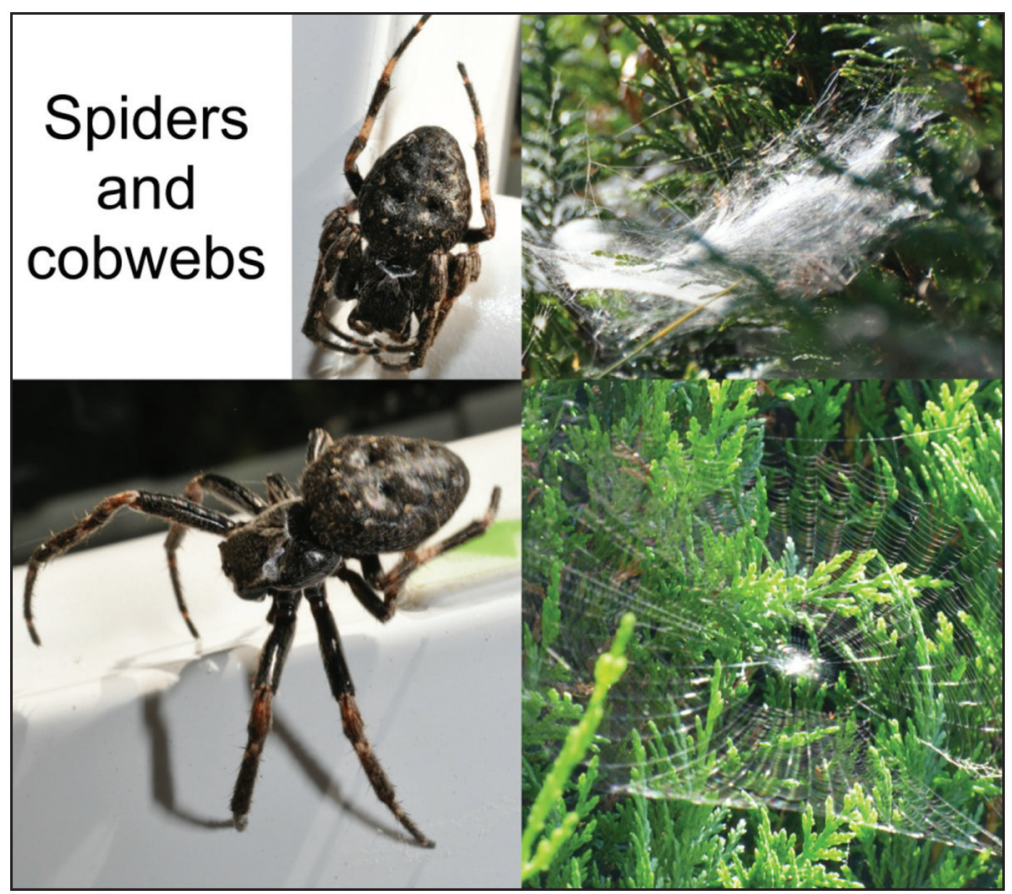

Figure 47. Spider and cobweb. Spider silk is stronger than steel, for the same weight. 
silk glands. The liquid which is produced hardens as it is squeezed outside the body to form silk fibers with outstanding properties. Nanomaterials mirroring the nature of spider silk would have many applications in sport equipment, aeronautics, automobile or household goods as well as in the defense domain, flak vests for example.

\section{Low-friction materials}

The skin of a shark exhibits very low adhesion and large drag reduction for fluid flow. The shark skin is covered with placoid scales (dermal denticles) arranged in a special geometry that reduces friction and turbulent flow during swimming. As a result a shark can reach speeds greater than $70 \mathrm{~km} / \mathrm{h}$.

Several applications have been developed mimicking the shark skin structure. Swimwear has been manufactured to minimize the water resistance. Paints based on nanoparticles have been developed to cover the external surface of boats. The paints are applied using a pattern imitating the structure of the shark skin.These paints also resist UV radiation, temperature and mechanical changes.

Shark skin has also antimicrobial properties and a new material using a a pattern alone derived from shark skin has been recently developed.

\section{Multiscale structures}

Many biological systems are made of self-assembled hierarchical structures over several size scales. This give them outstanding physical properties. This is the case, for example, for bones, wood, skin, teeth and the spider silk we discussed above. At the nanometer scale, there is always a structure which is involved to get excellent mechanical properties. In bones there are multiscale structures but also multiscale responses to deformation. Applying a multiscale philosophy to the development of advanced materials can lead to increased strength and desirable deformation characteristics.

\section{Summary}

Nature is a pool containing very beautiful examples of nanomaterials and nanostructures that lead to unique properties and capabilities. Thoroughly 
investigating these and understanding the underlying structures and the operational mechanisms which are responsible can spur important advances in the development of man-made nanomaterials providing analogous benefits. 


\section{Nanofabrication}

Nanofabrication techniques provide the ability to manufacture objects with details on the 1-100 nm lengthscale. Developing low cost manufacturing techniques with high throughputs and high yields is essential to facilitate wide-scale applications of nanotechnology.

\section{Top-down and bottom-up approaches}

There are two approaches to building nanostructures or nanomaterials. The "top-down approach" and "the bottom-up approach" (figure 48). In the top-down approach the starting point is a bulk material and nanostructures are built by removing part of that material or adding extra material to get the desired structure. In microelectronics, for example, the top-down approach is currently used to manufacture integrated circuits or memories. The starting bulk material is often a silicon wafer. This wafer can be patterned using lithographic techniques. As the required accuracy increases and the dimensions of the circuit decrease, these lithographic techniques become more and more complicated and expensive.

Top-down approaches are a natural evolution of manufacturing processes used at the macroscopic scale. Small systems, with a size at the $\mathrm{cm}$ or mm lengthscale, can be manufactured by skilled mechanics with lathes, saws and sanders. The difficulty with top-down approaches at the micron or nanometer scale is to get perfect surfaces and edges. It is essential to master processes giving a perfect control of the size of the details to be manufactured. Because some parts of the initial macroscopic structure are removed, top-down approaches require more raw materials and generate more waste than the bottom-up approach.

In the bottom-up approach, Instead of removing material from a bulk structure, one selectively organizes atoms or molecules to build the nanostructure. This is actually the way nature builds complex microscopic or macroscopic structures. The bottom-up approach has several advantages compared to the top-down approach. It requires less material than the top-down approach because there is no waste material lost, for example due to etching. It is easy to make thin films and it allows the creation of detailed structures smaller than those produced with photolithography. 


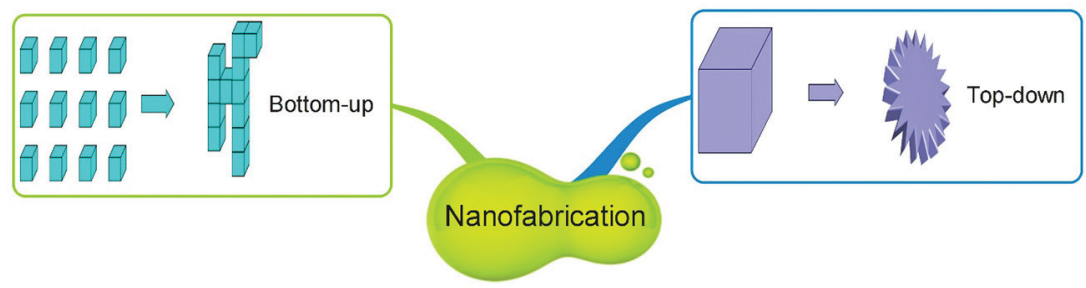

Figure 48. The two approaches to nanofabrication: the top-down and the bottom-up approaches.

The problem is that the number of atoms or molecules required to build a nano-object is very large and normally each atom or molecule cannot be handled individually. For example, suppose that we want to make a monolayer film (a film containing just one layer of molecules). If we use molecules with diameters of $0.2 \mathrm{~nm}$ and want to build a $1 \mathrm{~cm}^{2}$ monolayer film, we need to arrange $2.5 \times 10^{15}$ molecules. Placing each molecule one by one at a rate of 1 per second would require almost 80 years of work. This is clearly an important issue. In the bottom-up approach, it is necessary to have a method to build nanostructures where the molecules self-organize. Other challenges for the bottom-up approach are to be sure that the nanostructure which is built by self-assembly is assembled in the correct way and that that there is no contamination during the self-assembly process.

The most efficient way to manufacture complex nanosystems is to combine the top-down and bottom-up approaches. The use of templates, catalysts or applied force fields can allow directed self-assembly.

\section{Lithography}

Lithography is the corner stone of microelectronic fabrication and is the technique used to make microprocessors and memories. It is a technique in which a pattern is tranferred to a photosensitive material called photoresist or resist. A photoresist produces, under irradiation by light (UV or X-rays) or ions (electrons or ions) a negative or a positive image depending upon the nature of the resist (see figure 49). After exposure, there are physico-chemical changes in the exposed areas and the resist is developed, for example by immersion into a liquid. The principle of positive and negative resists is shown in figure 49. Positive and negative resists act in the same way as slides and negatives in the field of photography. A positive or negative image of the pattern on the resist is then transferred to another layer by etching or lift-off processes. 


\section{Positive resist process}
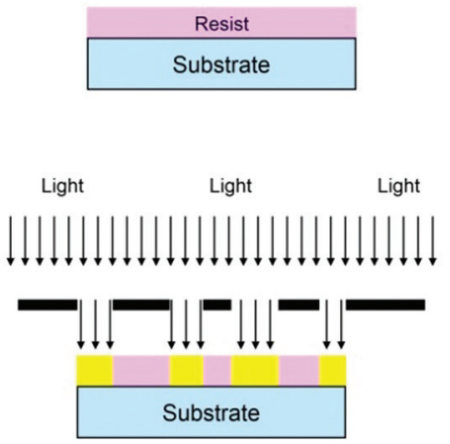

\section{Negative resist process}

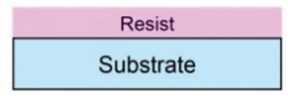

Exposure

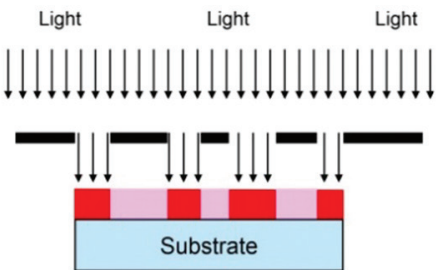

Resist development
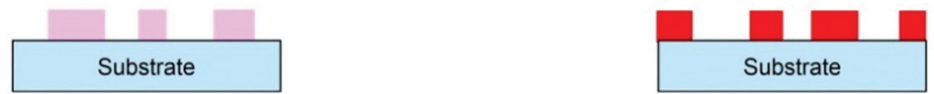

Figure 49. Principle of lithography using a positive and negative photoresist.

The semiconductor material most commonly used in microelectronics is silicon. Other materials such as $\mathrm{SiC}, \mathrm{GaAs}$ or $\mathrm{GaN}$ are also used for specific applications.The silicon is usually in the form of wafers, circular thin slices of material obtained from an ingot of a high purity (99.9999999\%) single crystal of silicon. The available size of silicon wafers has increased with time. It was 4" diameter (100 mm) around 1975 and $300 \mathrm{~mm}(11.8$ ) in 2001. The $300 \mathrm{~mm}$ technology is still used and technologists are bringing the $450 \mathrm{~mm}\left(18^{\prime \prime}\right)$ technology to the industrial stage. A larger size for the wafer allows the manufacture of more integrated circuits at the same time which decreases the cost of each piece.

Several steps, called mask levels, are necessary to manufacture an integrated circuit. The different operations in each stage are schematically illustrated in figure 50. The starting point of the lithography process is to deposit a thin layer of resist by spin coating. In order to do that the wafer rotates at high speed and the dissolved resist is poured onto the wafer. This allows deposition of a thin homogeneous layer of photoresist. In a second step, curing and baking of the layer gets it prepared for use. In the third step, the pattern of the mask of the integrated circuit is projected using a reduction optics system and this is repeated until the whole wafer is illuminated (step and repeat exposure). After exposure, the resist is developed 


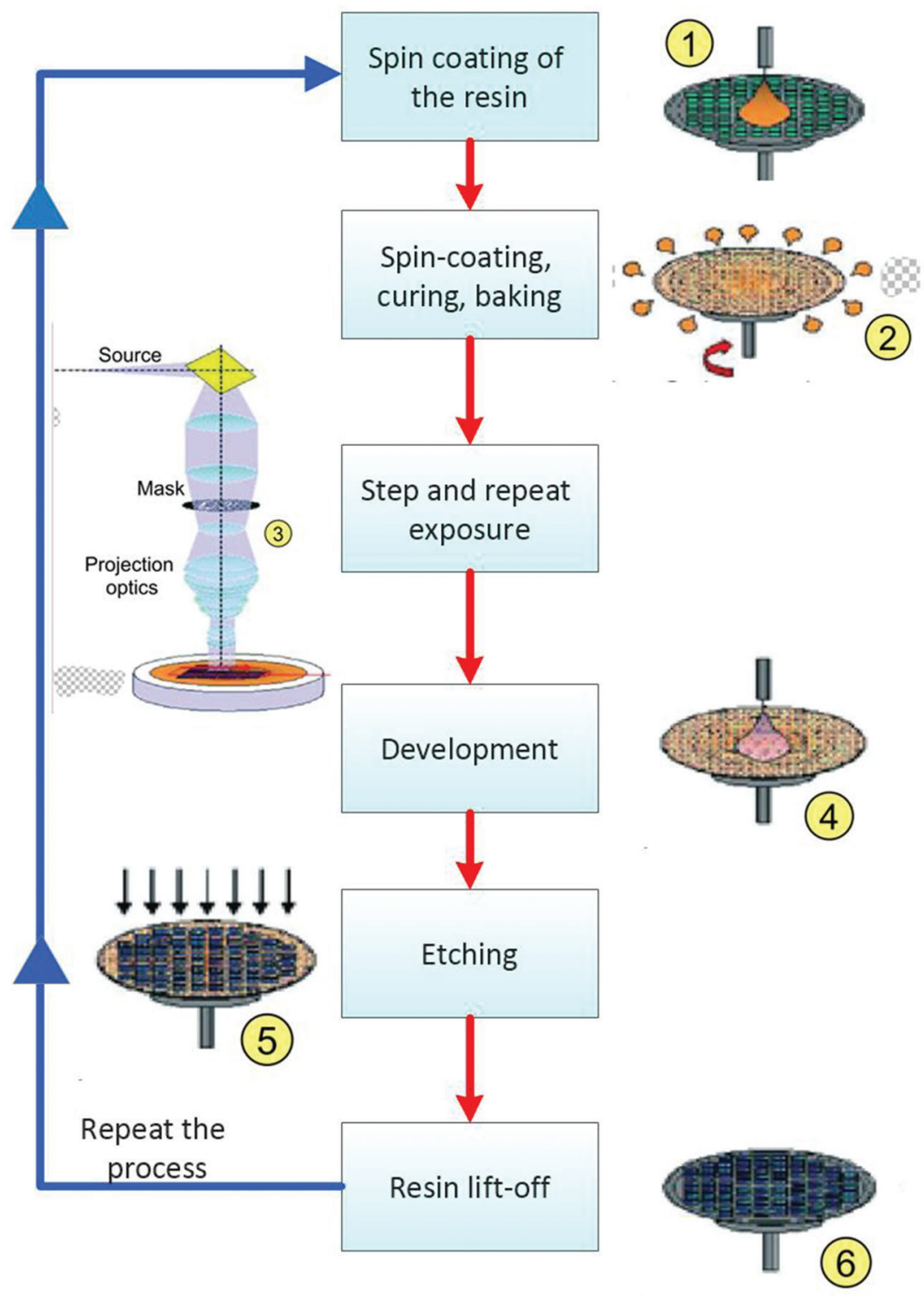

Figure 50. Different stages in a lithography process during one masking level. In the manufacturing of a microprocessor several masking levels are needed. Diagram built from a figure in Clef CEA $n^{\circ} 52$, courtesy of CEA/LETI.

and the parts of the resin which have been removed are etched. After this operation, the part of the resin that is left over is lifted-off before deposition of a next layer to start a new stage of lithography. Accurately positioning each mask level with respect to the previous ones is a critical issue. 
As the dimension of the details of the circuit decreases, the lithography techniques become more and more complicated and expensive.

All lithography operations have to be done in rooms with exceptionally clean environments. In these rooms, the number of dust particles in the air must be severely restricted. If dust deposits onto the wafer surface during manufacturing, the circuit will either malfunction or not function at all. Sources of light with short wavelengths have to be used (excimer lasers for example) and new techniques have to be developed to further increase the resolution achievable. As a consequence, the cost of lithography machines exponentially increases as the required accuracy in dimension decreases.

\section{Nanolithography}

Lithography, in the microelectronics domain, is progressing swiftly step by step. Each step is called a generation or technology node. For example, a 30 $\mathrm{nm}$ technology node, refers to the size of the details that one can design on a wafer, such as the size of the gate of a transistor or half the distance between two identical features (half-pitch) in the case of memories. In 1971, the technology node was at $10 \mu \mathrm{m}$ while in 2011 the technology node was $22 \mathrm{~nm}$, that is in the nanometer lengthscale. By the end of 2014, the $14 \mathrm{~nm}$ technology node has been used by Intel. Actually the $32 \mathrm{~nm}$ technology node was introduced 2010. In 2001, the technology node was $130 \mathrm{~nm}$ and in 2004 it was $90 \mathrm{~nm}$. It is around that period that microelectronics moved in the nanorange.

Light sources used in lithography have a given wavelength.The smallest wavelengths used are obtained from excimer lasers ( $\mathrm{KrF}$ at $248 \mathrm{~nm}$ and $\mathrm{ArF}$ at $193 \mathrm{~nm}$ ). To go down to smaller dimensions, extreme UV lithography has been developed using a very short wavelength $(13.5 \mathrm{~nm})$. However, at these dimensions, conventional optics does not work anymore and special techniques need to be used.

$X$-ray lithography is difficult and expensive to use because conventional lenses do not work and it is necessary to use mirrors to guide the beam. The mask is also an issue and usually a 1:1 image is made. Conventional $X$-ray sources can be used as well as synchrotron radiation.

Extrapolating microelectronics technology to smaller dimensions (below $100 \mathrm{~nm}$ ) also raises new problems if conventional materials are used. For example, despite the fact that a transistor consumes less energy and generates less heat when its dimensions are reduced, the number of transistors placed per unit area may increase dramatically. Therefore more and more heat must be dissipated per unit area and the question of heat dissipation becomes an important issue. 
In order to be able to etch details smaller than the wave length of the light source, special tricks have been developed such as high numerical aperture optics, immersion lenses, phase shifting masks, optical proximity correction and double patterning approaches. This is fine for microelectronics applications below the $100 \mathrm{~nm}$ range which are able to make huge investments. However, non-microelectronics applications cannot afford such high costs because they do not have a high volume production.

For non-microelectronics applications, it is necessary to use low-cost and flexible lithography techniques. Three of them are available: electron beam, ion and nanoimprint lithography.

\section{e-beam lithography}

Electron beam ( $e$-beam) lithography uses an electron beam to pattern directly the resist deposited on the substrate. As far as the process is concerned, it works the same way as light lithography that we just discussed. However no mask is required and the exposure is done by direct irradiation. A resolution of a few nanometers can be routinely obtained. $e$-beam lithography is used to design masks and templates used in light lithography. The main drawback of $e$-beam lithography is that it takes a long time to expose the substrate because the irradiation is done point by point, in raster mode, similar to that employed to direct the electron beam in a cathode ray tube. This technique can only be used in the laboratory, or for low-volume production of nanosystems.

In $e$-beam lithography, PMMA (poly-methyl-methacrylate) is a resist often used as a positive tone. Exposed areas to the electron beam become more soluble in the developer. An example of a negative resist is HSQ (hydrogen silsesquioxane) which undergoes crosslinking under exposure and becomes less soluble.

\section{Helium-ion lithography}

Lithography with ion beams is also possible. Scanning helium ion beam lithography (SHIBL) with subnanometer beams is an attractive technique for nanostructure manufacturing. This technique has been made possible by the development of the helium ion $\left(\mathrm{He}^{+}\right)$microscope for imaging. A resolution of about $0.35 \mathrm{~nm}$ can be obtained filling the gap between scanning electron and transmission electron microscopy. This capability can also be applied for 
manufacturing. The technique can be used not only for lithography but also in ion milling of nanosystems. Features with dimensions below $10 \mathrm{~nm}$ can be obtained. One advantage of an ion beam compared to an electron beam is the negligible back scattering of ions compared to that for electrons.

\section{Nanoimprint lithography}

Nanoimprint lithography is a low-cost technique with a high throughput. The resolution is moderate (for example $25 \mathrm{~nm}$ ) but it can be applied to large areas. The principle of thermal nanoimprint is shown in figure 51 .

To begin with, a mold (stamp) containing the negative image of the nanostructures we want to print is pressed into a thin film of resist cast on a substrate. The process is facilitated by operating just above the glass transition temperature of the resist, which is the temperature above which the resist becomes thermoplastic. The image of the mold is impressed onto the resist. This image can be made more precise using an anisotropic etching to remove the residual resist in the compressed areas.

Another nanoimprint technique consists in imprinting a liquid photocurable resist material at room temperature and low pressure. An external UV source is used to crosslink the resist. This technique, called ultraviolet assisted nanoimprint lithography, requires either a UV-transparent mold, or a UV-transparent substrate.

Three-dimensional nanostructures are interesting for biosensors, nanofluidic devices, photonic devices, etc. $e$-beam or $X$-ray lithography can be used but these methods are complex and expensive. Methods based on sophisticated nanoimprint are currently under development combining for example nanoimprint and $X$-ray lithography, or imprinting inorganic sol-gel materials. Nanoimprint lithography is an interesting alternative to conventional lithography to fabricate nanostructures. This technique is already in use to produce commercial microlenses. One promising application is 3 -dimensional nanopatterning.

\section{Dip-pen nanolithography}

Dip-pen nanolithography uses the tip of an atomic force microscope (AFM) coated with a chemical or biological compound to pattern the surface of the substrate. The tip of the cantilever of the AFM plays the role of the pen, the chemical or biological product that of the ink, and the substrate the role of the paper. 

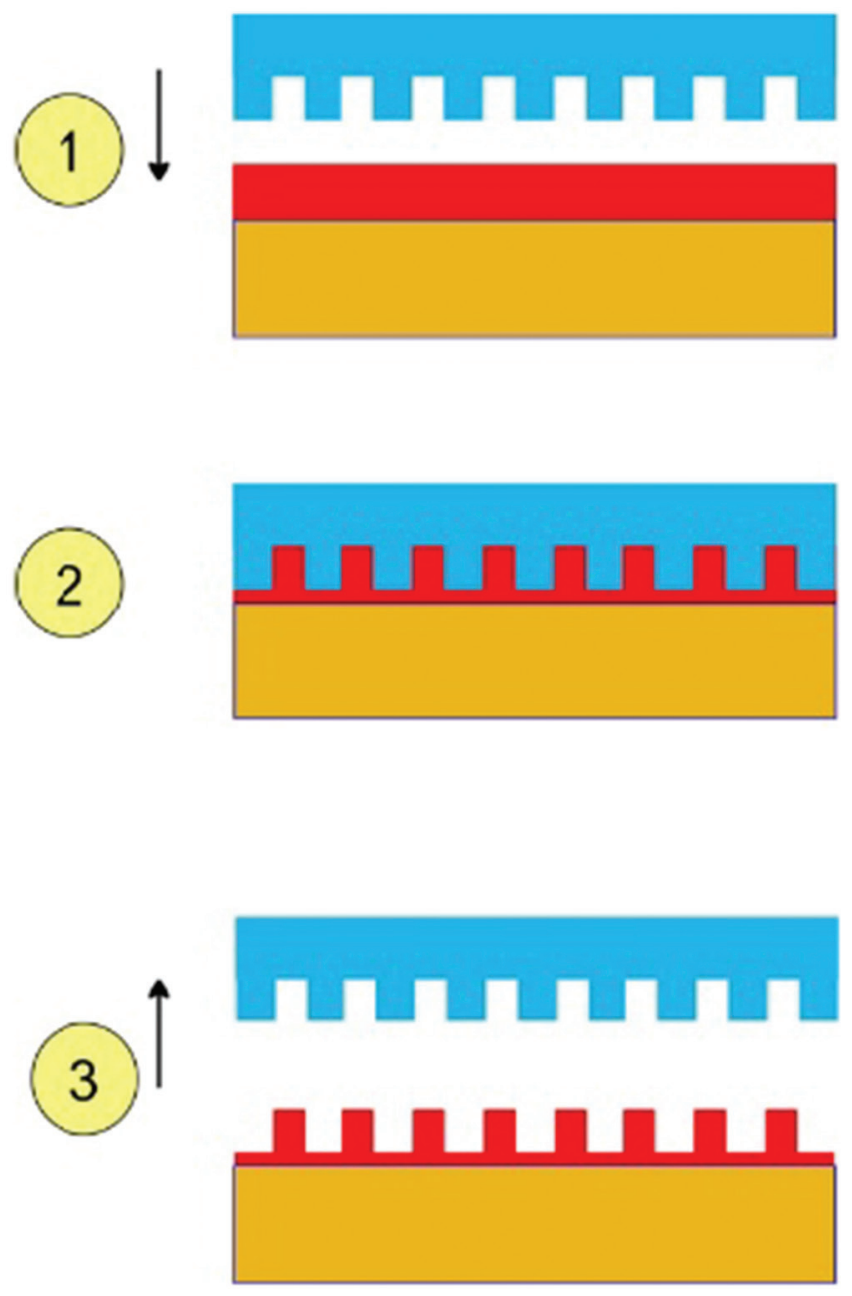

Figure 51. Principle of thermal nanoimprint lithography.

One-dimensional and two dimensional arrays of cantilever allow to have parallel processing which increases the throughput. Dip-pen arrays with more than a million tips have been made. Pen lithography can be improved with different method and other techniques exist such as polymer pen lithography or beam-pen lithography.

\section{Deposition at the nanoscale}

Several methods exist to fabricate thin films with nanoscale thicknesses. 


\section{Atomic layer deposition}
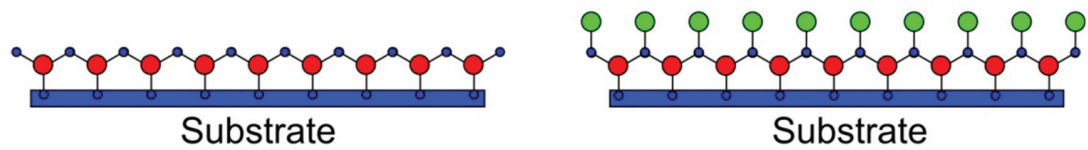

Figure 52. Principle of atomic layer deposition (see text).

\section{Atomic layer deposition}

Atomic layer deposition (ALD) is a a chemical deposition technique to fabricate pinhole free and conformal (the thickness is the same everywhere on the surface of the substrate) nanometer thick films on a substrate. The substrate is not necessarily a flat surface. It can have a complex geometry. ALD requires two reactant gases (precursors) which are sequentially introduced into the reaction chamber containing the sample. The precursors react with each other to form an atomic layer.

The principle of ALD is schematically shown in figure 52 for a single cycle corresponding to the fabrication of a one atomic layer film. A cycle consists of four different steps where the two precursors, A and B are successively involved:

1 The first precursor (A) mixed with a carrier gas (nitrogen or argon, for example) is introduced into the reaction chamber containing the sample. It is chemically absorbed on the surface of the substrate until the surface is saturated.

2 The excess of precursor A is removed by sweeping the volume of the chamber with the carrier gas.

3 Precursor B is pulsed into the reaction chamber and reacts with the absorbed molecules. The quantity is large enough for the surface to be saturated.

4 The excess of precursor B is swept away by the carrier gas and by pumping.

This cycle can be repeated several times to get a film of desired thickness.

\section{Self-assembled monolayers}

The self-assembled monolayer (SAM) technique can be used to create thin films on a surface of a metal or semiconductor, or on the surface of 


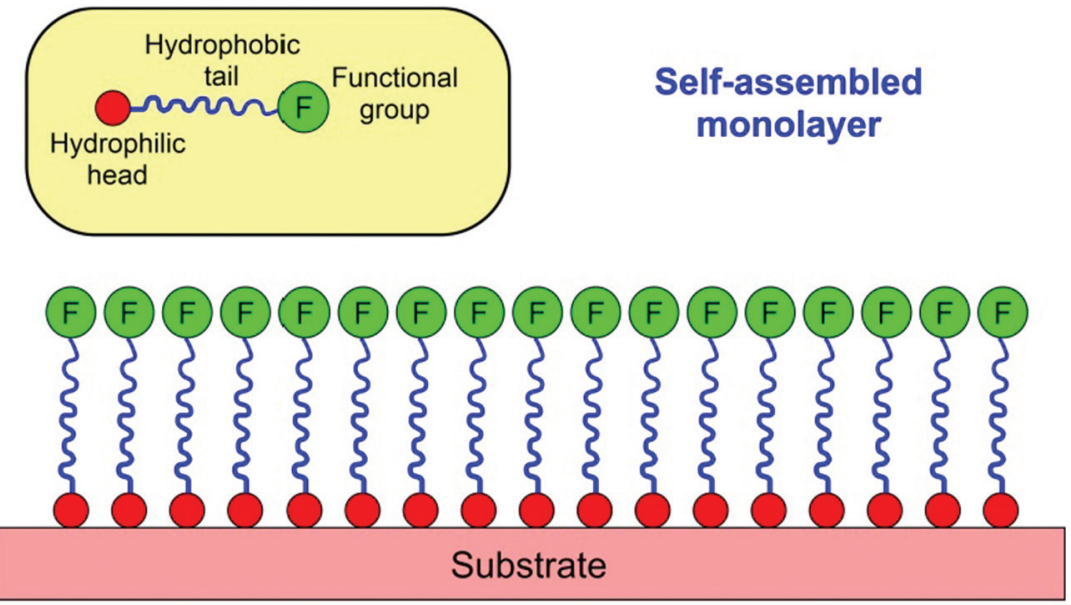

Figure 53. Illustration of a self-assembled monolayer.

nanoparticles using molecules with a hydrophilic head and a hydrophobic tail (amphiphilic molecules). A self-assembly process occurs because of the chemisorption of the hydrophilic head of the molecules on the surface of the substrate. After a time ranging from minutes to hours, a crystalline or semicrystaline structure can be formed on the surface of the substrate.

A schematic display of a SAM is shown in figure 53. As we can see in this schematic drawing, the molecules can even be functionalized.

Use of catalysts or applied stresses may allow for anisotropic or directed self-assembly. Of course the most efficient way to manufacture complex nanosystems is to combine the top-down and bottom-up approaches.

\section{Covalent bonds}

There is a great practical interest in developing covalent bonds between a molecule and a surface because covalent bonds provide a strong anchorage to the surface. If a layer of polymer is simply deposited onto a surface and is attached by weak forces such as Van der Waals or hydrogen forces, it is easy to remove part of the layer during use. On the other hand, if it is anchored to the layer by covalent bonds, it is more resistant to external physical or chemical conditions because a much larger amount of energy is required to break the covalent bonds. Covalent bonds can be used to functionalize a surface 


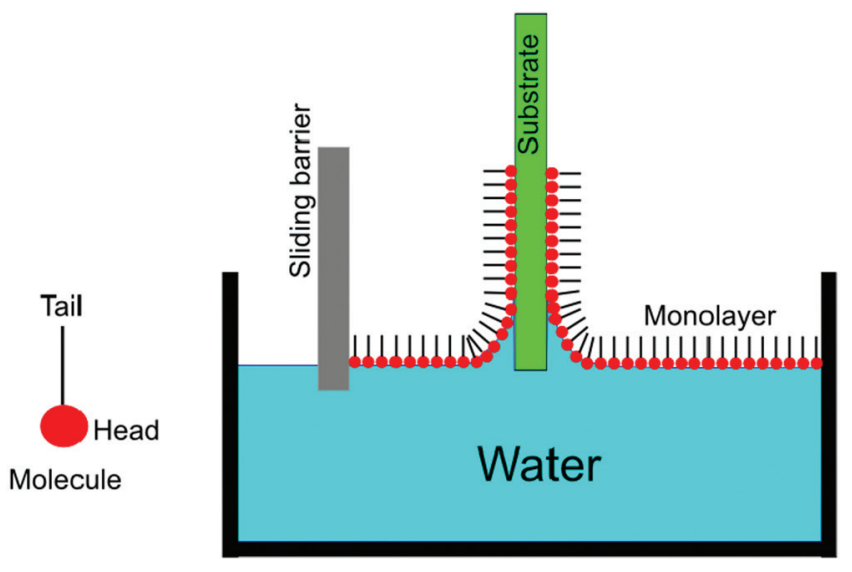

Figure 54. Principle of the preparation of a Langmuir-Blodgett film.

or protect it. Several methods have been developed to create covalent bonds on a layer. They are often based on stimulating the surface to facilitate a chemical reaction between the surface and the molecules to be anchored. This stimulation can be exposure to light, heat, electrical potentials, etc. Metals, silicon and carbon are examples of useful substrate materials.

Covalent bonds can also be formed with an already deposited layer (Langmuir-Blodgett films, SAMs, etc.). One of the advantages of anchoring covalent bonds on a nanothickness layer made by self-assembly is that the geometrical ordering of the first layer is used to build the second one.

\section{Langmuir-Blodgett films}

Langmuir-Blodgett films consist of one or more monolayers of an organic material. The organic molecules which are used usually have a hydrophilic head (polar group) and a hydrophobic tail. The principle of fabrication is shown in figure 54. Organic molecules are put onto the water surface at a concentration such that they are not closely packed. A sliding barrier is used to closely packed them onto the surface. A substrate, immersed in water, is moved up slowly and a monolayer deposits onto its surface. The process can be repeated to form multilayers either with the same molecules or with different molecules.

The Langmuir-Blodgett technique is a typical example of bottomup approach by self organization. Multilayers from two to hundreds of monolayers can be fabricated by this method. 


\section{Polishing, etching, patterning}

\section{Chemical mechanical polish}

Clean polished surfaces are usually required for thin film deposition. The aim is to get a flat, smooth surface without defects. Historically, a number of polishing techniques have been used to obtain such surfaces. Polishing materials have been used for centuries to manufacture copper and bronze mirrors and more generally glass optics. More recently, water polishing has been used in wafers used in integrated circuit fabrication.

Chemical-mechanical polishing (CMP), sometimes called planarization, combines both mechanical and chemical polishing. It was developed first by IBM in the 1980 s for microelectronics. A wafer is pressed against a rotating pad with a polishing slurry. Polishing slurries contain mechanically active abrasives and chemically active components. Abrasives are generally hard oxide particles suspended in the slurry. Depending on the polishing work to be done, abrasive particles have a diameter ranging between $10 \mathrm{~nm}$ and $10 \mu \mathrm{m}$. Active chemical components, dissolved in water, are substances that soften and oxidize the surface which is polished. After polishing, cleaning of the surface is necessary to eliminate residual chemicals and particles.

As an illustration of CMP, we shall consider the damascene process applied to copper. For a long time the electrical interconnections in integrated circuits have been made with aluminum. The interconnects were produced by substractive etching of a film of aluminum using a photoresist pattern. To improve the performances of microprocessors, it has been necessary to replace aluminum by copper. Copper has a lower resistivity than aluminum allowing better performance and a lower joule heating, thus higher current densities. It also leads to better overall reliability. The main issue with copper is the difficulty in patterning the interconnections with conventional etching techniques. Furthermore, copper quickly diffuses into oxides and silicon, and quicky oxidizes in air. In the 1990s, IBM introduced the Damascene process to form copper inetrconnections in integrated circuits. The principle of the Damascene process is shown in figure 55 .

In the Damascene process, a dielectric is first deposited on the wafer and then etched according a photoresist pattern. A barrier is then deposited to prevent a mixing of materials above and below the barrier. This gives the substrate shown on top of figure 55. Copper is then deposited, by electroplating for instance. Finally the surface is planarized by CMP which removes the unneeded copper. 


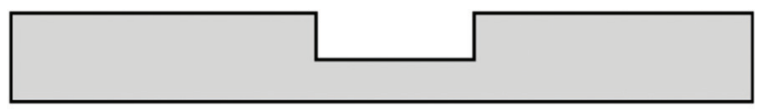

\section{Substrate}

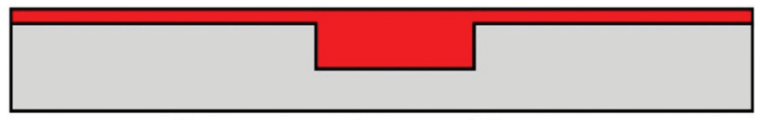

\section{Copper deposition}

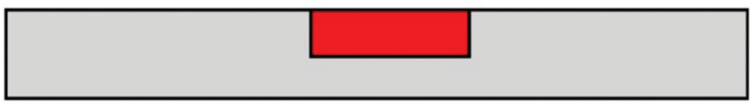

\section{After polishing}

Figure 55. Principle of the damascene process.

\section{Summary}

Developing and mastering different technologies to manufacture nanomaterials and nanosystems is essential in the development of nanotechnology. A large number of methods adopted from different fields are available and used in this area. Manufacturing can proceed in a "top-down" approach in which a nanosized system is obtained from a larger system, or in a "bottom-up" approach where it is built from elementary pieces. The two approaches can be combined for greater efficiency. The main concern is to develop economical high throughput techniques for large scale production of systems containing nanostructured parts. 


\section{King carbon}

Organic chemistry is the chemistry of carbon compounds (other than sim-

ple salts such as carbonates, oxides, and carbides). It is a domain in its own right because of the huge importance of organic compounds in the living world. Until 1985, only three forms (allotropes) of pure carbon were known. These were graphite, diamond and lonsdaleite (which is a diamond with a hexagonal lattice instead of a cubic lattice). Other allotropic forms exist but are less known. In 1985, the discovery of new allotropes of carbon opened a vast field in nanotechnology because of outstanding properties of these new forms of carbon.

\section{Fullerenes}

In 1985, R.Curl, H.Kroto and R.Smalley, inspired by the reported observation by Canadian radio astronomers of long linear chain molecules of carbon in interstellar space, carried out an experiment designed to simulate the conditions under which carbon nucleates in the atmosphere of red giant stars. By vaporizing graphite and condensing the vapor in an atmosphere containing an inert gas they discovered a new form of carbon. Several different clusters were identified. The simplest one contained 60 atoms of carbon arranged as a truncated icosahedron as shown in figure 56 . The structure of this hollow molecule is similar to that of a soccer ball with 20 hexagons and 12 pentagons. Each carbon atom is bound to 3 neighboring carbons atoms by covalent bonds. It should be noted that two pentagons have no common edge. The discoverers named the molecule buckerminsterfullerene because of the similarity of its structure to that of the geodesic domes designed by the architect Buckminster Fuller for the 1967 Montreal world exhibition. Other designations such as buckyball or simply $\mathrm{C}_{60}$ are used to identify this spherical molecule which has a diameter of about $0.7 \mathrm{~nm}$.

The $\mathrm{C}_{60}$ molecule is highly symmetric and remains invariant under 120 symmetry operations. The $\pi$-electrons are delocalized over the whole surface making this molecule particularly stable (breaking a $\mathrm{C}_{60}$ molecule requires temperatures over $\left.1,000^{\circ} \mathrm{C}\right)$. The delocalization of $\pi$-electrons over the surface of the sphere is somehow an extension of the delocalization of $\pi$-electrons over a hexagonal benzene molecule. Solid $\mathrm{C}_{60}$, a yellow powder, turns pink when dissolved in toluene. 


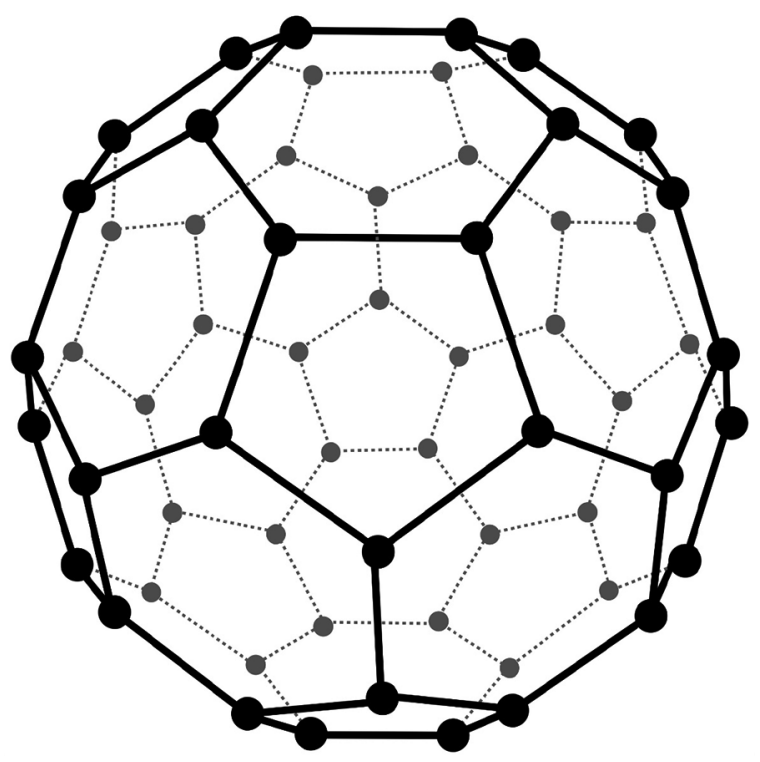

Figure 56. Schematic picture of a C60 molecule., also called a "Buckyball".

Other carbon clusters were also found In the condensed mixture, but the buckyball was the smallest stable molecule. The clusters belong to a larger family of molecules called fullerenes made only of carbon atoms and having a hollow shape (sphere, ellipsoid, tube). They have an even number of carbon atoms: $\mathrm{C}_{60}, \mathrm{C}_{70}, \mathrm{C}_{76}, \mathrm{C}_{78}, \mathrm{C}_{82}, \mathrm{C}_{84} \ldots$. The discoverers of fullerenes were awarded the 1996 Nobel Prize in chemistry for this work.

Actually, fullerenes structures had already been predicted by E.Osawa in 1970, well before their discovery in 1985. But their importance was not appreciated. Fullerenes are exceptionally interesting molecules with many potential applications. The existence of a hollow shape provides the capability to encase an atom or another molecule. Figure 57 illustrates this schematically showing a noble-gas atom caged inside the $\mathrm{C}_{60}$. The interest of putting another entity inside the $\mathrm{C}_{60}$ structure is that it is protected against external interactions. In health care applications,for example, this could allow delivery of a drug to the right place in the body before it is released.

Another possibility illustrated in figure $5^{8}$ is to functionalize the surface by adding specific chemical groups. It is possible to graft a molecule on the surface to make compound molecules that are more hydrophilic (water-loving) or lipophilic (fat-loving) than the initial fullerene. It is also possible to tailor the electronic or optical properties. Figure 58 represents such a fullerene. This molecule has potential applications in the catalyst domain or in non-linear optics. 


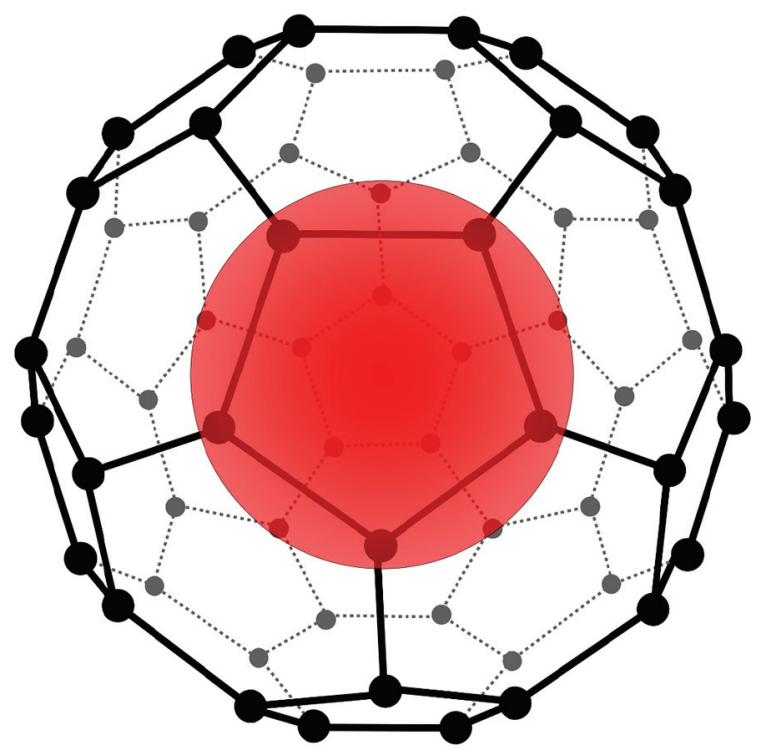

Figure 57. Schematic picture showing an atom of a noble gas caged within a C60 molecule.

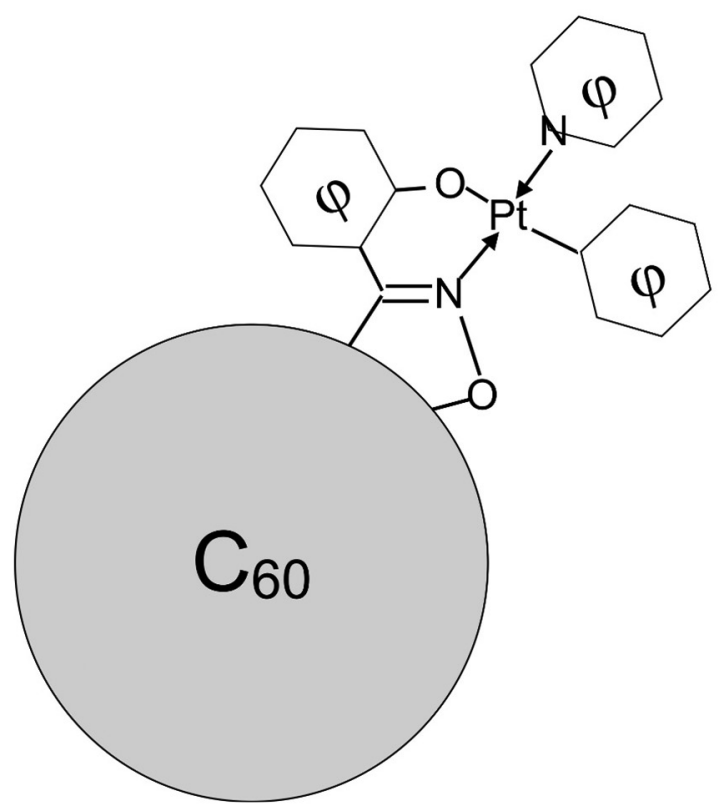

Figure 58. Isoxazoline fullerene chelating agent. 


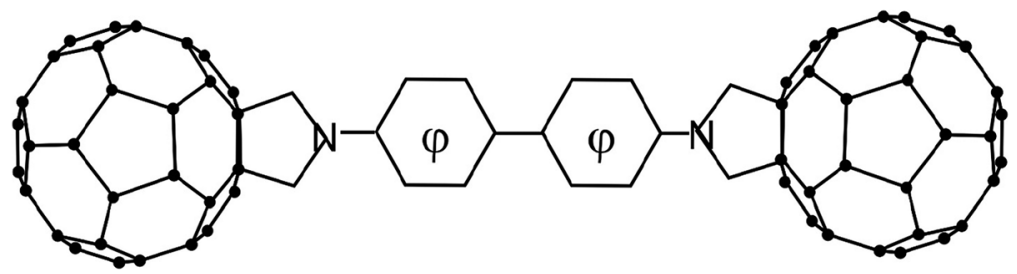

Figure 59. Schematic drawing of a dumbbell fullerene.

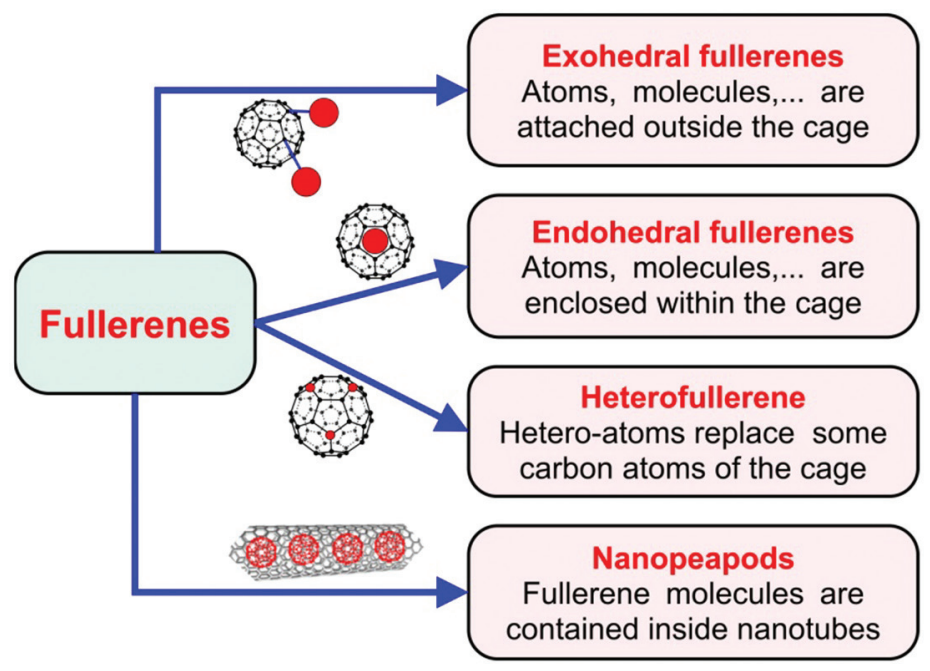

Figure 60. Different types of engineered fullerene molecules.

Many possibilities are open and complex structures with specific properties can be synthesized. For example, figure 59 shows two $\mathrm{C}_{60}$ molecules connected by a molecule with delocalized electrons.

Many types of modified fullerenes can be synthesized for potential applications (see figure 6o).

Exohedral fullerenes, such as that in Figure 6o, are synthesized in a chemical reaction between a fullerene and another molecule or atom which can be grafted to the outside of the fullerene. This is the most important family. As fullerenes are insoluble in water, adding a proper chemical group can make the resulting exohedral fullerene soluble.

It also is possible to enclose an atom in the hollow of fullerene (endohedreal fullerene). When the atom trapped inside is a metal, the molecules are called metallofullerenes. The size of the atom enclosed must be small enough to fit in the hollow. Most of the endohedral molecules synthesized are made out of large size fullerenes: $\mathrm{C}_{82}, \mathrm{C}_{84} \ldots$ Atoms such as scandium, lanthanum, yttrium and light rare gases have been caged inside fullerenes. 
In the litterature, they are usually denoted by a hybrid formula such as $\mathrm{La} @ \mathrm{C}_{82}$, for example, which means that an atom of La is caged inside $\mathrm{C}_{82}$.

In heterofullerenes, one or more carbon atoms of the cage are replaced by hetero-atoms such as nitrogen, for example.

Nanopeapods are single-walled carbon nanotubes with buckyballs inside. They are potentially interesting for molecular electronic devices.

Fullerene derivatives have many potential applications. Fullerenes are inert and are not absorbed when administered orally in a water-soluble form. Water-soluble fullerenes have a very low acute toxicity and are excreted by the kidney. They have many potential applications in biology and medicinal chemistry. For example, endohedral metallofullerenes can be used as magnetic resonance or $X$-ray imaging contrast agents. Some modified fullerene molecules are utilized as radiopharmaceuticals. They can be used as tracers or to kill cancerous tumors. Another application is the use of the cytotoxicity of $\mathrm{C}_{60}$ water-soluble derivatives (carboxylic derivatives) when exposed to light. The cytotoxicity results from the ability of the product exposed to light to cleave DNA. This also can be utilized in cancer treatment.

Thanks to the delocalized $\pi$-electrons on the surface, fullerene molecules have the ability to strongly interact with free radicals (chemical entities with unpaired electrons). It has been shown the $\mathrm{C}_{60}$ is able to interact with 34 methyl radicals, $\mathrm{CH}_{3}$. Fullerene can thus play the role of an anti-oxidant and free radical scavenger, useful in the medical domain.

\section{Graphene}

Graphene is a monolayer of carbon atoms packed in a honeycomb lattice (figure 61). It is a two-dimensional structure formed of hexagons in which all the vertices are occupied by carbon atoms. The distance between two neighboring carbon atoms is $0.142 \mathrm{~nm}$. This is a bit larger than in benzene $(0.139 \mathrm{~nm})$. It was isolated for the first time in 2004 by A.Geim and collaborators at Manchester University. In 2010, A.Geim and K.Novoselov won the Nobel price in physics for their work on graphene.

Interestingly, in the 1930s, graphene had been predicted by famous theoretical physicists to be thermodynamically unstable at finite temperature. In fact, graphene is stable and can be produced with several methods. The stability is due to $3 \mathrm{D}$-ondulations (ripples) of the $2 \mathrm{D}$ lattice.

Graphene has outstanding physical properties. Although just an atomic layer thick it is extremely strong (about 200 times stronger than steel of the 


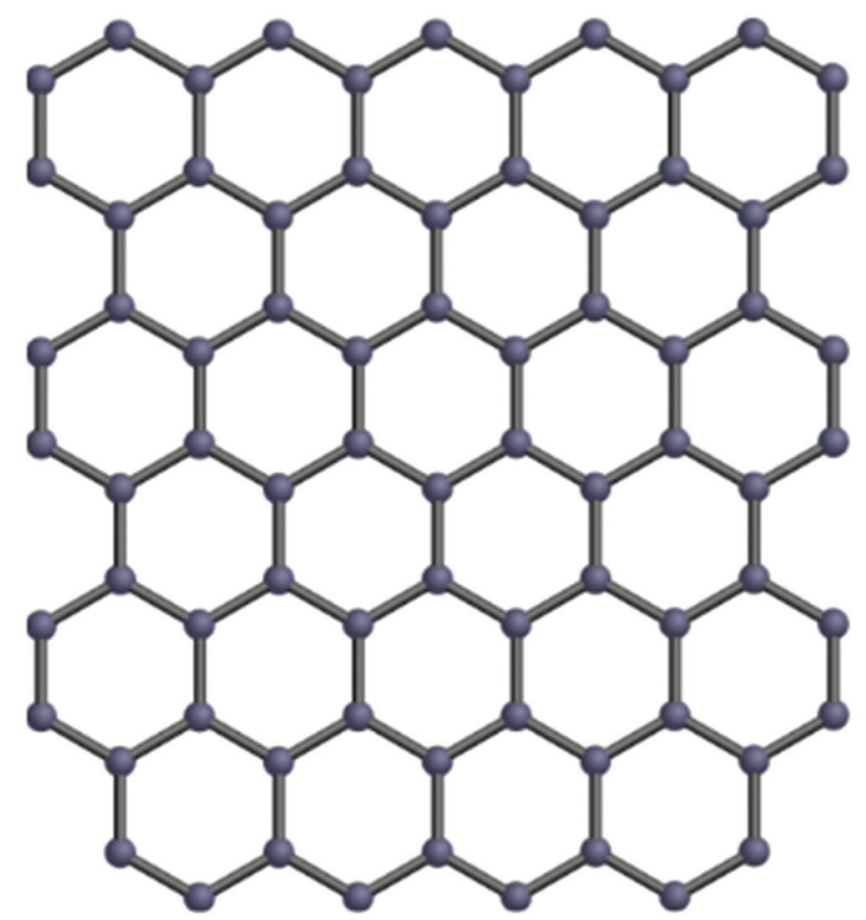

Figure 61. Schematic drawing of graphene. Image from Wikimedia Commons (http://commons. wikimedia.org) originally from Openclipart.

same dimensions, but 6 times lighter). It is an excellent conductor of electricity and heat. Compared to silicon, extensively used in microelectronics, its electrical conductivity is much higher, it is much less brittle and highly flexible.

Novoselov and collaborators first manufactured graphene by peeling a graphite crystal with an adhesive tape (scotch-tape method) and transferring it onto a colored and oxidized silicon wafer of thickness smaller than $300 \mathrm{~nm}$. This peeling method is adequate for laboratory studies but cannot be generalized to mass-production.

In top-down approaches, graphene sheets can be produced by separation, peeling, cleaving or exfoliation (mechanical or electrochemical) of graphite and derivatives. These methods usually have low yields and require great investments. Bottom-up approaches are more appealing for mass-production. CVD (chemical vapor deposition) and epitaxial growth on various substrates which allow preparation of large quantities of graphene sheets (mono or multilayers) is preferred. Other bottom-up approaches such as arc discharge, chemical conversion, carbon nanotube unzipping, self organization of surfactants, etc. are also used. 


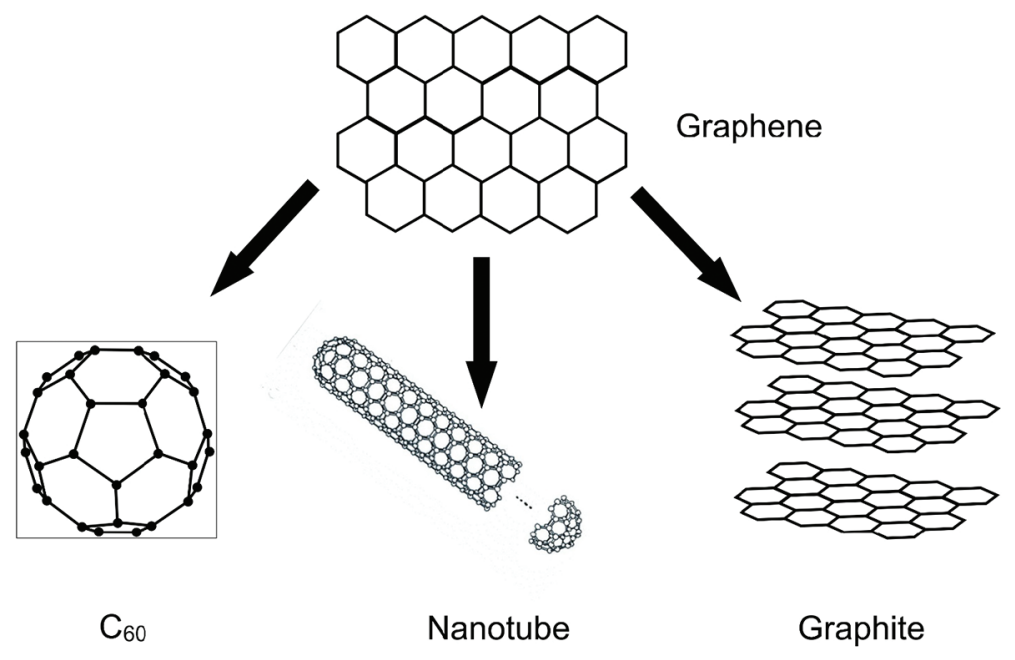

Figure 62. Carbon species that can be derived from graphene. Nanotube image courtesy of CEA/ LETI (France).

Graphene belongs to the family of two-dimensional (2D) materials which have been extensively investigated because of their unusual physical properties compared to $3 \mathrm{D}$ materials. Some of them, such as layered metal dichalcogenides or copper oxides, exhibit high-temperature superconductivity. $2 \mathrm{D}$ materials can be used to make sandwich structures constructed from several different layers of such materials. These $2 \mathrm{D}$-heterostructures can be tailored with atomic precision and the whole structure can be tailored to fulfill several applications.

\section{Carbon nanotubes}

The honeycomb lattice of carbon atoms in graphene can be formally considered as the basic structure from which other carbon species are derived (figure 62). Graphite ${ }^{8}$ is a stack of graphene layers, carbon nanotubes are rolled-up cylinders of graphene and $\mathrm{C}_{60}$ needs the introduces pentagons into the hexagonal lattice of wrapped graphene.

In 1991, S.Iijima discovered nanotubes of carbon in the soot produced in a machine dedicated to $\mathrm{C}_{60}$ production. They had been observed previously but not pursued. For example, in 1952, carbon nanofilaments with an inner cavity have been identified with a transmission electron microscope. 


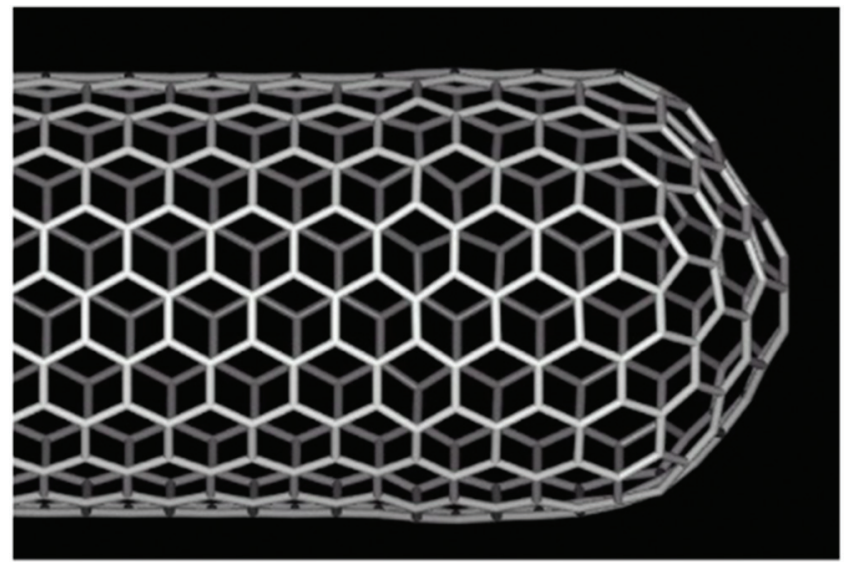

Figure 63. Schematic picture of a closed single-walled carbon nanotube. Image courtesy of CEA/ LETI (France).

Carbon nanotubes are cylindrical tubes built from one or more sheets of graphene which are a few nanometers wide with lengths ranging from below a micrometer to several millimeters. Two families can be distinguished: single-walled carbon nanotubes and multi-walled carbon nanotubes.

- A single-walled carbon nanotube is a part of a graphene sheet wound on itself in a cylindrical manner. It can be eventually closed at the end by a fullerene-like the hemisphere (figure 63). Single-walled carbon nanotubes are often produced in tight bundles.

- A multi-walled carbon nanotube is made of several concentric single-walled carbon nanotubes. The distance between two sheets of graphene is of the order of $0.34^{-0.36 ~ n m}$. This is about the distance separating two layers of carbon in graphite. The diameters of multi-walled nanotubes are between a few nanometers and tens of micrometers.

The ends of nanotubes can be open or closed (figure 63). Since empty space is present inside the nanotube, atoms or molecules can be trapped inside (the nanopeapods in figure 60 are an example).

According to the classification presented in Chapter 4, carbon nanotubes are $1 \mathrm{D}$-nanomaterials with a diameter in the nanometer range and a length reaching several micrometers. They have outstanding mechanical properties. They are as stiff as diamond and their high strength is used to make fibers. The mechanical elastic modulus is for instance 5 times that of steel. Furthermore, their thermal conductivity is eight times that of copper, similar to that of diamond. Carbon nanotubes are currently incorporated 
into commercial products such as sporting goods, automotive parts, rechargeable batteries, etc. For example, the winning Tour de France bicycle in 2005 was made of a carbon nanotubes composite.

Several methods have been developed to produce carbon nanotubes in bulk quantities. They can be produced at high temperature with a graphite rod placed in an inert atmosphere. High temperatures are produced using an electric arc, laser ablation or a solar beam. They can also be produced at medium temperatures with catalytic chemical vapor deposition. More than 100 companies around the world manufacture and sell carbon nanotubes and this number increases regularly. Hundreds of tons of carbon nanotubes are produced each year and this amount is also constantly increasing. The production capacity by 2015 is expected to be over 9,00o tons. The largest installed capacity is found in the Asia-Pacific region.

Single-walled nanotubes with a purity greater than $95 \%$. are commonly produced using an arc discharge process The nanotubes have a diameter between 0.7 and $2 \mathrm{~nm}$ and form a bundle of about $8 \mathrm{~nm}$. They have an excellent mechanical strength and very good thermal and electric conductivity. They are used in several applications: chemical sensors, nanobiomaterials, conductive heating films, conductive nano-ink, display, etc.

Multi-walled carbon nanotubes are produced with thermal chemical vapor deposition and a purity greater than $95 \%$ is reached. Their diameters range from 10 to $30 \mathrm{~nm}$. They are employed in a large number of applications such as: chemical sensors, conducting paints, polymer and metal composites, etc.

There are actually several ways to build a nanotube from a sheet of graphene. It can be made, in a "gedanken" experiment, in the same way as we curl a sheet of paper with a hexagonal lattice drawn on the paper.There are an infinite number of ways to fold a graphene sheet into a nanotube structure and the result is characterized by different helicities. This leads to three different families of nanotubes called: zigzag, armchair and chiral nanotubes (figure 64).

If the sheet is rolled along one of its the symmetry axis one can obtain either a zigzag or an armchair nanotube (figure 65). In all the other cases we get a chiral nanotube (asymmetric in such a way that the structure and its mirror image are not superimposable).

To characterize the nature of a nanotube built from a graphene sheet, it is convenient to define an origin and two unit vectors $\mathbf{a}$ and $\mathbf{b}$ (in brown in figure 64). A vertex on the lattice can be located by two numbers $m$ and $n$. The vector $m \mathbf{a}+n \mathbf{b}$ goes from the origin to the vertex. A carbon nanotube referred as $(n, m)$ means that the sheet of graphene has been rolled up in 


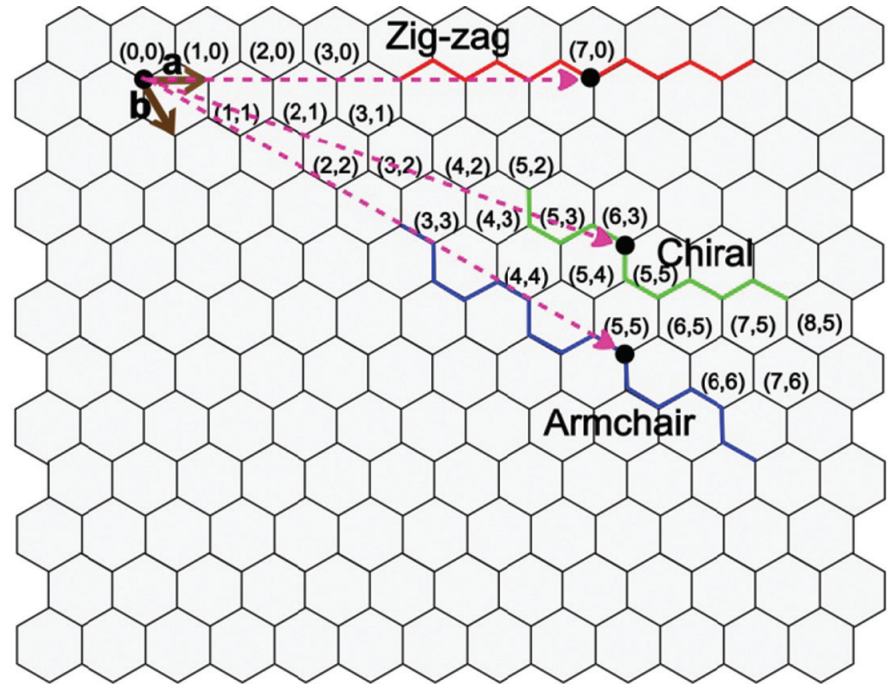

Figure 64. Graphene sheet from which a single-walled nanotube is made by rolling up the sheet (see text).

\section{Axis of symmetry of the cylinder of the nanotube}

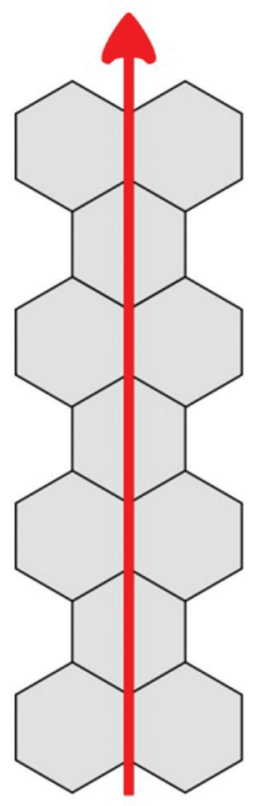

Zig-zag

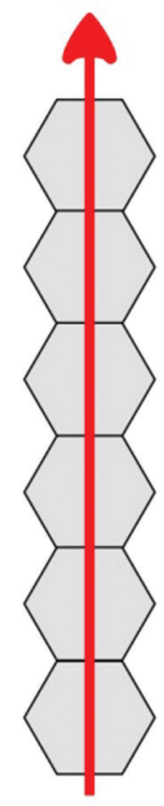

Armchair

Figure 65. Difference between a zigzag and an armchair nanotube. The sheet of graphene is rolled up around the indicated symmetry axis. 
such a way that the atom located in $(o, o)$ is superimposed on the atom located in $(n, m)$. If $n=m$, we have an armchair nanotube. The physical properties strongly depend upon the way the graphene sheet is rolled. For example, armchair nanotubes behave as metals.

A carbon nanotube has a metallic behavior if $2 n+m$ is a multiple of 3 . This is the case of armchair carbon nanotubes because $n=m$. Zigzag carbon nanotubes can behave either as a metal if the previous relationship is satisfied (for example, for $(n, m)=(12,0)$, or as a semiconductor. Metallic nanotubes are, in principle, 1,00o times better conductors than copper but this property can be reduced by impurities or defects.

Multi-walled carbon nanotubes are good electrical conductors. They can be used in STM, AFM and in other probe instruments such as the electrostatic force microscope. With carbon nanotube tips, better quality images are obtained compared to those made from etched Si or metal.

Carbon nanotubes can also be used in chemical or biological sensors. Indeed the electrical resistivity changes on exposure to ambient air containing $\mathrm{NO}_{2}, \mathrm{NH}_{3}$ or biomolecules.

Aligned nanotubes can be produced by various methods with or without catalysts. Figure 66 shows an example of a carbon nanotube forest obtained in a CEA laboratory. They can have several applications such as membranes for water filtration, high-performance electric wires, etc.

Carbon nanotubes can contain atoms or molecules inside their cylinders. They can also be functionalized with other molecules giving them new properties. For example functionalized carbon nanotubes have been developed in the USA to detect and destroy an aggressive form of breast cancer (HER2-positive breast cancer) which does not respond well to chemotherapy or hormone treatment. Attaching an anti-HER2 antibody to short carbon nanotubes provides the ability to detect and destroy malignant cells using a near infrared laser at two wave lengths (one for detection, one for destruction of the cancer cell to which the carbon nanotube is attached).

\section{Summary}

The discovery of fullerenes, carbon nanotubes and graphene has opened a new field of investigations with many real and potential applications. Fullerenes and carbon nanotubes turn out to have outstanding and useful properties in many fields. They can also be used as a basic component, grafting on their surfaces chemical groups with specific physical, biological 


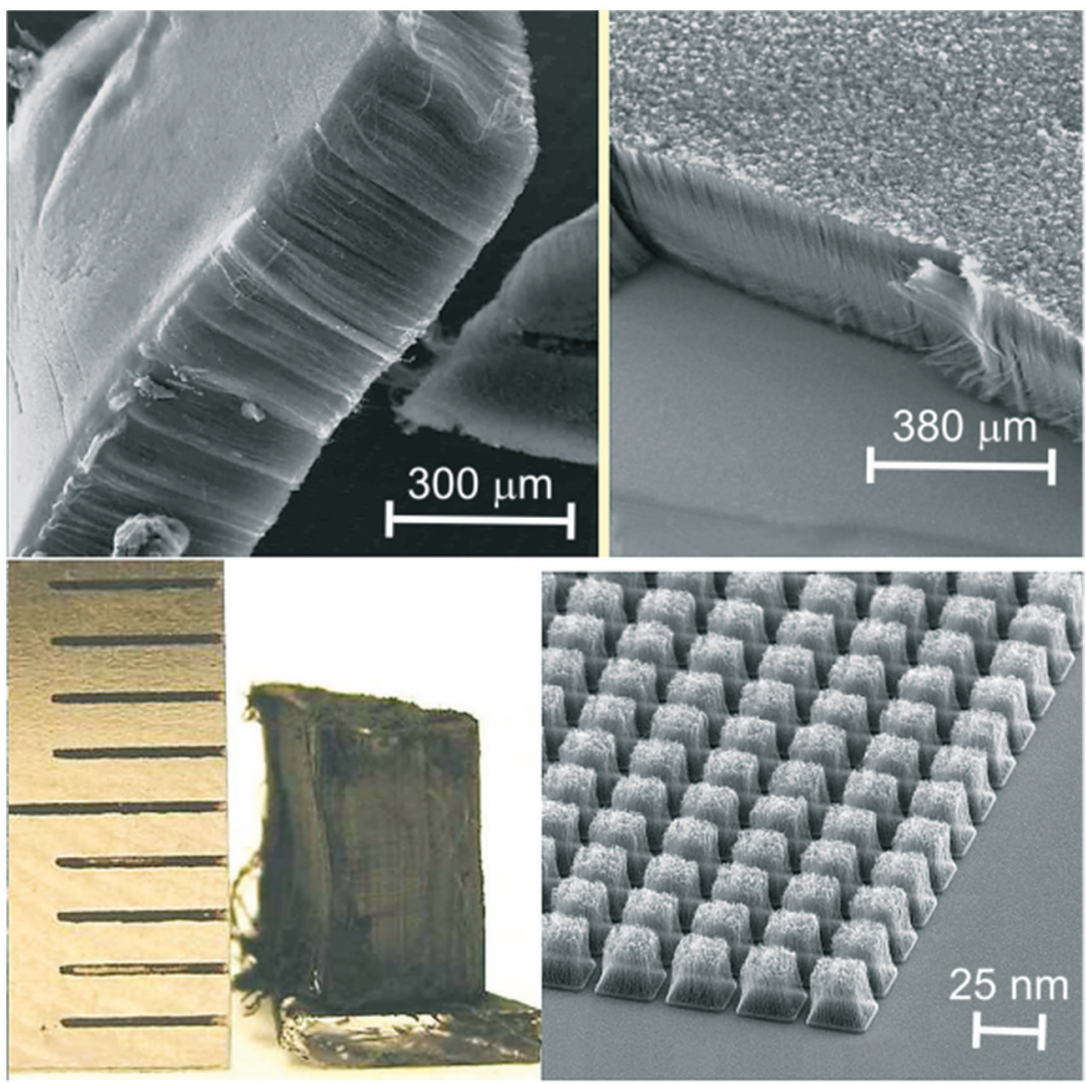

Figure 66. Manufacturing of carbon nanotube forests. In the top left part, a monolayer forest is shown. The top right part displays a multilayer forest. The bottom left part shows a forest of ultra long (>5mm) nanotubes and finally the bottom right hand part of the figure displays a forest featuring controlled localized growth. Image courtesy of CEA/LETI (France).

or chemical properties. Furthermore, they can be to used protect active molecules during their travel to a target. Applications encompass fields as different as the medical sector, the catalysis domain and composite materials, for example. The ability to produce nanotubes with specific properties: diameter, number of walls, defects, chirality, inclusions etc. allows tailoring of these tubes to the desired application. Graphene is also a promising material for micro and nanoelectronics. 

Part 2 Applications of nanotechnology 



\section{Health Diagnostics}

Improvements in life style and progress in medicine have been responsible for a large increase of life expectancy during the last two centuries. Today, in developed countries, people live much longer than they did in previous eras. In France, for example, the life expectancy before the French revolution (1789) was less than 30 years. It reached $5^{\circ}$ years in 1900 and has consistently improved since that period. Today it is above 80 years. In underdeveloped countries, the life expectancy is lower and can be of the order of 40 years in the poorest countries or in those where large segments of the populations are faced with a diseases such as AIDS or alcoholism, for example. As populations become older deaths due to chronic diseases increase. About $80 \%$ of seniors have at least one chronic disease and $50 \%$ have at least two. In the United-States $70 \%$ of all deaths are due to chronic diseases. Technological advances such as those offered by nanotechnology can have far reaching consequences in healthcare.

The main issue in the health domain is to provide an affordable and efficient medicine that all can access. For our discussion we can classify health care can be classified into three main areas: diagnostics, therapeutics and restoration or regenerative medicine (figure 67).

Proper medical diagnoses usually require some combination of analysis and imaging. The current trend is to employ non-invasive or minimally invasive techniques to look inside the human body or to make specific analyses on samples of material taken from the patient. Diagnostics techniques should be as painless as possible so that they can be performed routinely and used to identify threats of serious disease. In the case where a sample is needed to perform an analysis, it is also desirable that the volume of the sample be as small as possible.

Once a disease or health disorder is identified, it is necessary to treat the patient in the most efficient way. In the therapeutics stage it is important to deliver the right quantity of drugs to the right place and at the right time in order to minimize side effects and increase treatment efficiency. Monitoring the efficiency of the treatment using minimally invasive diagnostic methods is also required.

Accidents or diseases can damage or destroy parts of the human body. Further, as people live longer and their bodies age, repair or replacement of components such as organs, bones or teeth may become necessary. The third domain of medicine, restorative and regenerative medicine, addresses such needs. 


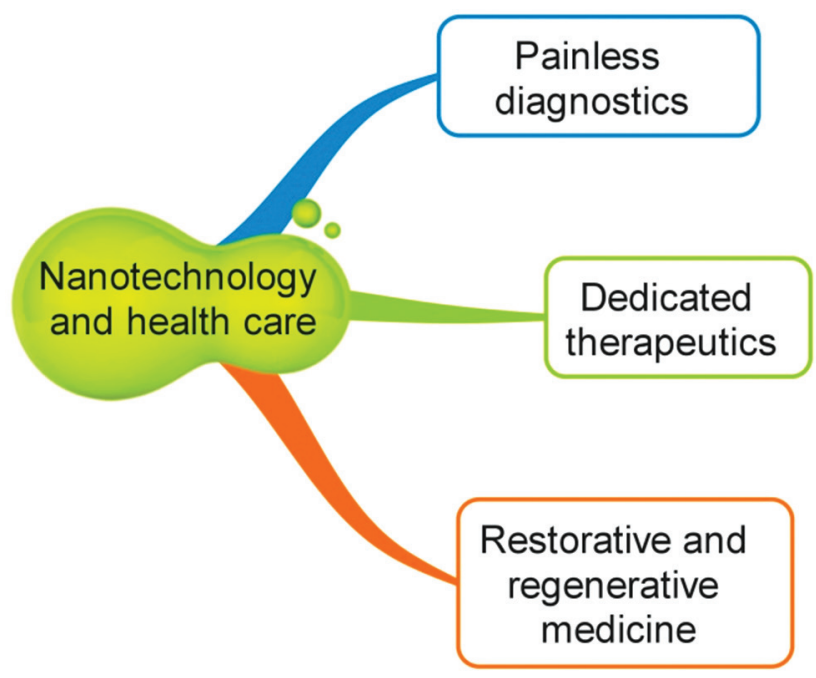

Figure 67. Nanotechnology can have impact in the three domains of medicine shown.

Nanotechnology can play a role in each of the health domains specified by providing improved diagnostics enabling early detection of or prevention of diseases and making more efficient techniques for treatment and follow-up monitoring available.

\section{Major diseases}

While there are many health risks and all must be addressed, it is possible to categorize the major diseases that affect very large proportions of the human population. All have a large social and economic impact. These are shown in figure 68.

The leading cause of death in developed regions of the earth such as the United States and The European Union is cardiovascular disease. Cancer ranks as the second leading cause of death. Neurodegenerative diseases such as Alzheimer's or Parkinson's are a serious problem. Due to dietary and lifestyle factors Type II diabetes is an increasing health problem. Inflammatory and degenerative joint diseases as well as musculoskeletal disorders lower the quality of life of many people. These diseases demand a long-term medication. Bacterial infections require new strategies to complement antibiotics because some bacteria are becoming resistant to them. For economic reasons, little research is now devoted to antibiotics. Fighting against viral infections also requires novel treatments. Both bacterial and viral infections require quick detection. 


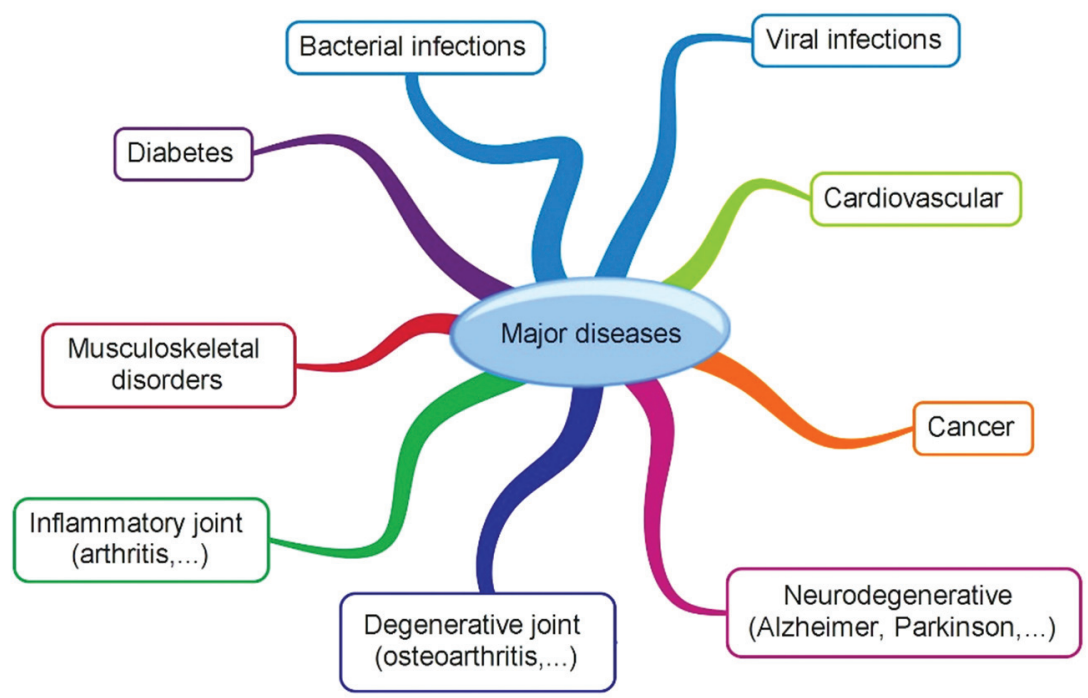

Figure 68. Major diseases touching a large part of the population.

\section{Diagnosis}

Diagnosis is the first step in identifying a disease or a health disorder. Diagnostic techniques should be specific, reliable, fast and accurate. The risks of "false positives" or "false negatives" at the borderline of the detection threshold should be minimized as much as possible.

Diagnostics include both in vitro techniques and in vivo techniques. Medical imaging is an example of an in vivo technique because it is performed directly on the patient. A blood analysis is an example of an in vitro technique since it is done on a sample taken from the patient. An important issue is to have non-invasive or minimally invasive techniques for both types of techniques.

\section{Imaging}

Imaging techniques which allow the investigation of a patient anatomy and, in some cases metabolic processes, are exceptionally powerful diagnostic tools (Figure 69). Functional imaging provides the ability to watch an organ function in real time. It allows the study the kinetics of biochemical and physiological phenomena. More recently molecular imaging allows visualizing the cellular function, genes and proteins directly or indirectly. They provide an unparalleled ability to identify dysfunctions of parts of the 

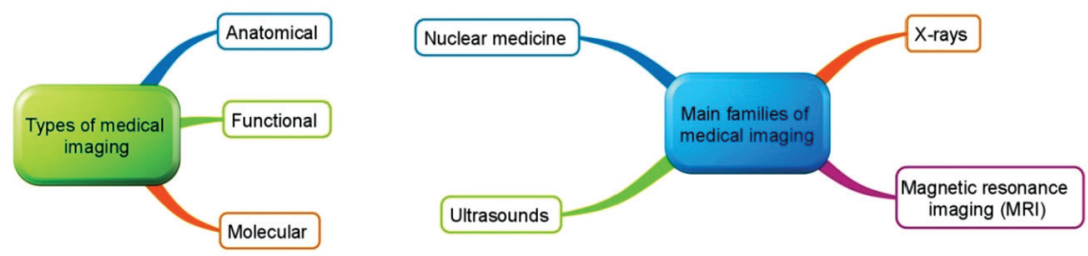

Figure 69. Types of applications of medical imaging (left hand side) and main families of medical imaging techniques (right hand part).

body and to anticipate appearance of disease. Modern Imaging techniques are highly accurate thanks to improvements in detection methods and computerized image treatment. The latter also allows most images to be digitized, leading to better storage and access to the data. While attempts are made to be as non-invasive as possible, these techniques sometimes require tracers to be injected into the patient.

The families of medical imaging which are mostly used are also indicated in figure 69 .

The use of X-rays is an old technique, but has improved greatly in our era. The aim is to decrease as much as possible the irradiation dose administrated to the patient and get the best spatial resolution.

Magnetic resonance imaging is a field in strong development with the advantage of being painless to the patient. Ultrasound techniques are increasingly being in many pathologies or to follow pregnant women. Nuclear medicine imaging techniques encompasses techniques based on radioisotope decay. The most common are scintigraphy, requiring injection or ingestion of a radioisotope, SPECT (Single-photon emission computed tomography) or PET (positron emission tomography).

It is often necessary to use several imaging techniques to make a diagnosis because they reveal different or complementary aspects of the health disorder.

The introduction of nanotechnology into imaging techniques through the development of new microprocessors, detectors and electronics is a natural evolution of imaging techniques. However, at the same time nanotechnology is playing a key role in providing new methods to enhance diagnostic imaging. The use of nanoparticles (which have large surface areas relative to their volumes) to deliver imaging agents to the desired site in the body, provides the ability to achieve a great density of interaction sites for interaction with molecular agents or particles. This can result in stronger imaging signals that can be detected outside the body of the patient. Dedicated nanoparticle structures with attached proteins or other molecules can detect indicators of disease at an early stage. It is also possible 
to create multifunctional products which can be used in different imaging techniques. Functionalized nanoparticles can have both a diagnostic and therapeutic potential. This property is often referred as theranostic.

A few examples of the use of nanoparticles in imaging:

- Gold nanoparticles are used in research to detect colorectal cancer, especially those which are difficult to see on colonoscopy. The detection is based on Raman spectroscopy. They are particularly useful because their surfaces resist oxidation and can be functionalized with different molecules.

- Iron oxide nanoparticles coated with dextran, a complex polysaccharide made of many glucose molecules, are used to target lymph nodes in diagnostic magnetic resonance imaging (MRI) and to detect cancer spread. These nanoparticles are taken up in the lymph nodes by normal cells but not by cancer cells. A MRI of the patient is performed before injection of nanoparticles and a second one is done 24 hours later. Lymph nodes that accept the nanoparticles become black while cancer cells stay bright.

- A new tumor detection technique, photo-acoustic imaging employs carbon nanotubes or gold nanoparticles which have been modified to emit sounds when appropriately stimulated by laser light. This light is used to heat the particles which produce an ultrasound signal that can be detected outside the body of the patient. The temperature reached is kept moderate in order not to hurt the tissue.

Structures in which gold nanoparticles are deposited electrostatically onto the surface of a PLA (polylactic acid ${ }^{9}$ ) microcapsule allow ultrasound contrast imaging. This structure has the potential to operate as a theranostic agent as well because it is also a photo hyperthermia agent allowing photothermal therapy in cancer treatment.

\section{In-Vitro Diagostics}

Presently diagnostic work typically has to be done in specialized laboratories and obtaining results can require hours or days. Required sample sizes can be large. Nanotechnology has an important role to play in the whole diagnostic process. The use of miniaturized laboratories, such as lab-on-chip systems which are in development and based on microelectronic techniques, can quickly provide a result using a very small amount of sample. Using smaller samples of body fluids or tissues makes the analysis 


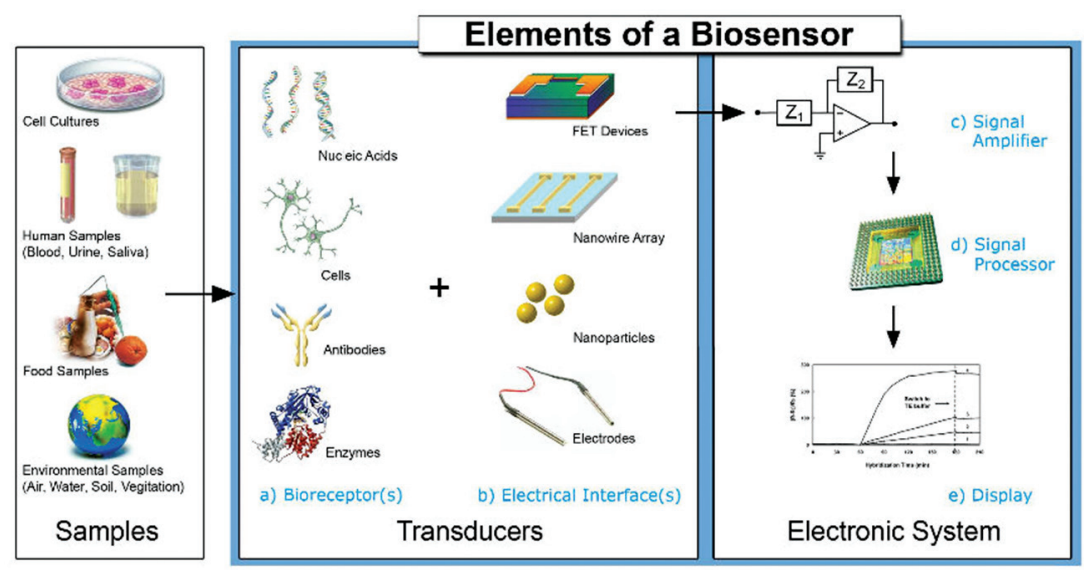

Figure 70. Biosensor system and components. Image from Wikimedia Commons (http://commons. wikimedia.org). Author Dorothee Grieshaber.

less invasive for the patient. The analysis can be done in a serial manner as it is done today, but also in parallel manner using arrays of miniaturized detectors when possible. Miniaturized analysis systems, including nanoscale components, will provide faster, cheaper results while producing less waste. Such low cost high speed analysis systems will soon become routinely available. The final goal is to make these capabilities directly available at the doctor's office.

\section{Biosensors}

Sensors are analytical devices which are able to recognize a specific chemical or biological species present in a sample, for example in a small quantity of blood from a patient. When the sensing is based on biomolecular recognition, the sensor is called a biosensor. There are several different types of biosensors based on different techniques of recognition such as antibody/antigen, enzyme based, hybridization with a strand of DNA, etc. The main elements of a biosensor are displayed in figure 70. The transducer transforms the signal resulting from the interaction between the analyte and the biological element into a signal which can be easily measured. This signal is amplified, processed and visualized by an electronic system.

A simple example of biosensor based on a cantilever is shown in figure 71. The free molecules are supposed to bind specifically to the molecules attached on 

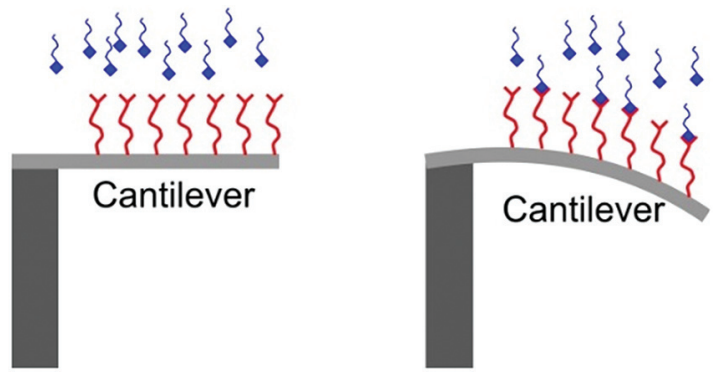

Figure 71. Principle of cantilever detection. The free molecules are supposed to bind to the molecules attached on the cantilever. When this happens the weight on the cantilever increases and it bends. The bending can be measured, by an optical method for example.

the cantilever. When this happens, the cantilever bends and information can be obtained from the measurement of the bending of the cantilever.

Nanoparticles of various types; gold or silica nanoparticles, quantum dots, fullerenes, etc. can be used in biosensors. Biological molecular species acting as sensors are attached to the surface of the nanoparticles. By the usual biological lock-and-key mechanism, they are able to detect the molecules of interest. The signal generated by the recognition mechanism can vary: a change of color, a change of mass, a change of emission properties etc.

Carbon nanotubes and nanowires can also be used in biosensors to detect a wide range of biological and chemical species. Viruses or proteins can be detected using these sensors which are often based on a change of electrical conductivity and employ semiconducting materials.

Although most of the biosensors developed from nanoscale materials are still in the laboratory, it is expected that they will enter operation at the patient scale during the next decade.

Microarrays of biosensors can be manufactured using the techniques of microelectronics. They can be used as diagnostic devices for DNA analysis, protein detection, virus detection, etc. Microarrays can consist of hundreds of biosensors acting independently and allowing specific detection of different bio-analytes within a mixture. Such microarrays can be used as screening tools, a function especially useful in new drug development.

Figure 72 introduces three families of microsystems containing nanocomponents which are under development. 


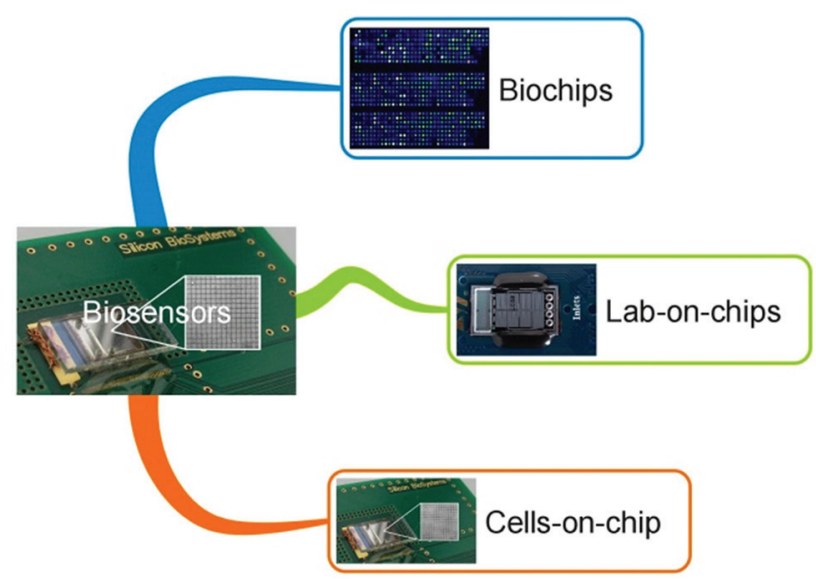

Figure 72. Different families of microsystems based on micro and nanoelectronics techniques and dedicated to diagnostics. The small images shown are courtesy of CEA/LETI (France).

\section{Biochips}

Genetic biochips (DNA-chips) are microarrays of sensors with the ability to perform a large number of analyses at the same time. Each sensor consists of a short strand of DNA. DNA-chips are able to quickly decode genes. When they are put into a solution to be analyzed, the analytes hybridize with the strands of DNA fixed on the microarray. A DNA-chip consists of thousands different nucleotide sequences arranged in a two-dimensional matrix. Biochips have been used to identify the genes of human DNA or of other living species. They can be used to detect the genes expressed by a cell or a tissue giving the ability to detect breast or prostate cancers, for example. They can also be used to detect proteins and in toxicological studies.

In some cases, the concentration of DNA in a sample is not always large enough to be detected safely or perhaps to be detected at all. There does exist a powerful technique, the Polymerase Chain Reaction technique, PCR, which can actually multiply the initial pieces of DNA to be detected: A single strand or a few strands of DNA can be multiplied by several orders of magnitude using PCR. In this in vitro technique thousands to millions of copies of a DNA sequence can be obtained. This amplification makes the DNA visible to the analysis system. 


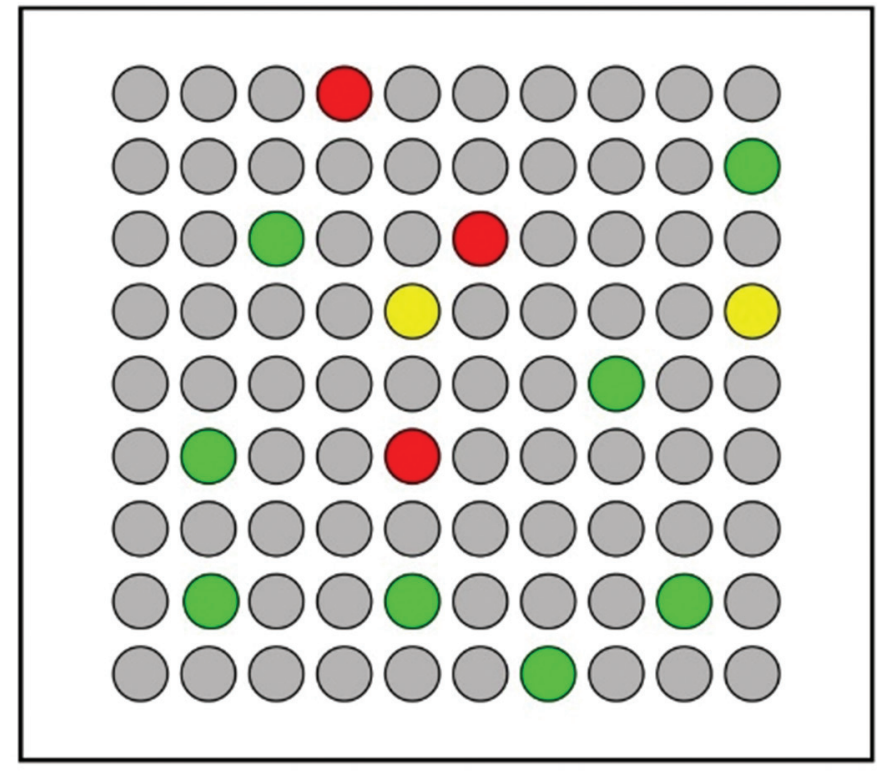

\section{No Hybridization}

\section{Hybridization from normal tissue}

\section{Hybridization from cancerous tissue}

\section{Hybridization from normal and cancerous tissue}

Figure 73. Schematic illustration of a DNA-chip.

PCR is performed in a vessel containing the DNA target to be amplified, a large concentration of olinucleotides (called primers) specific to the sequence investigated, the DNA polymerase and a mixture of the 4 bases which are the DNA building blocks. The PCR proceeds through a sequence of cycles, each of them comprises 3 steps lasting a few minutes: The cycle is repeated about 40 times, each time doubling the amount of DNA. About 100 billion copies can be obtained after 40 cycles. This process can be completely automated. Unlike some previous techniques it does not require the presence of bacteria.

Biochips such as these are used to detect oral cancers, for example. An oral cancer is usually discovered by dentists and oral surgeons and requires a biopsy of the cheek. The sample is sent to a laboratory and the result is received several days later. A nano-biochip recently developed in the US permits a painless test and the result is readily obtained in a quarter of an hour. Early detection of malignant or premalignant lesions is important 
because early treatment leads to a larger probability of survival. Early detection accords a 5 -year survival of $90 \%$. This figure drops down to $60 \%$ if the lesions are detected later.

Figure 73 explains schematically the way a DNA-chip is used in a small array. There are different spots indicating the probes hybridized on the array. Colored spots indicate that the probes have been hybridized. In this illustration it is supposed that red and green correspond to genes of the cancerous and normal tissue, respectively. Yellow spots correspond to a hybridization of both cancerous and normal tissue. This is because there are many probes located at a node of the array and not a single strand of DNA.

\section{Labs-on-chips}

Labs-on-chips, also called micro-total-analysis systems, integrate several chemical and biological analytical functions on the same chip. These devices are made of micro and nanocomponents manufactured with micro-electronic techniques. Labs-on-chip manipulate tiny volumes of biological fluids to perform analyses similar to those made in conventional laboratories. Miniaturized sensing systems and fluid control are essential to these devices.

Labs-on-chips are not simply miniaturized versions of conventional macroscopic laboratory techniques. They actually exploit the possibilities of new physical phenomena which appear at small length scales. Since the volumes are very small, for example in the nanoliter or femtoliter range, liquids behavior is different from that exhibited on the macroscopic scale as discussed in chapter 3. For example, since fluid dynamics is dominated by laminar flow, it is possible to create concentration gradients in nanoscale devices which are not achievable at the macroscopic scale. A good understanding of microfluidics and nanofluidics is essential to the development of devices performing similar analyses to those made in conventional laboratories, but much faster and at a substantially lower cost. Small samples and less reagent volume are required to perform the analysis. This produces less waste and, in the case where the sample is taken from a patient, is less intrusive. Since the volume analyzed is small and compact, the analysis is fast because the diffusion distances are much smaller than in macroscopic samples. As with biochips, it is possible to parallelize the measurements and build an array of labs-on-chips to get a high output rate. A mass production of labs-on-chips is possible using micro-electronic techniques. This lowers the cost substantially and could provide disposable chips. 


\section{Design considerations}

\section{Advantages}

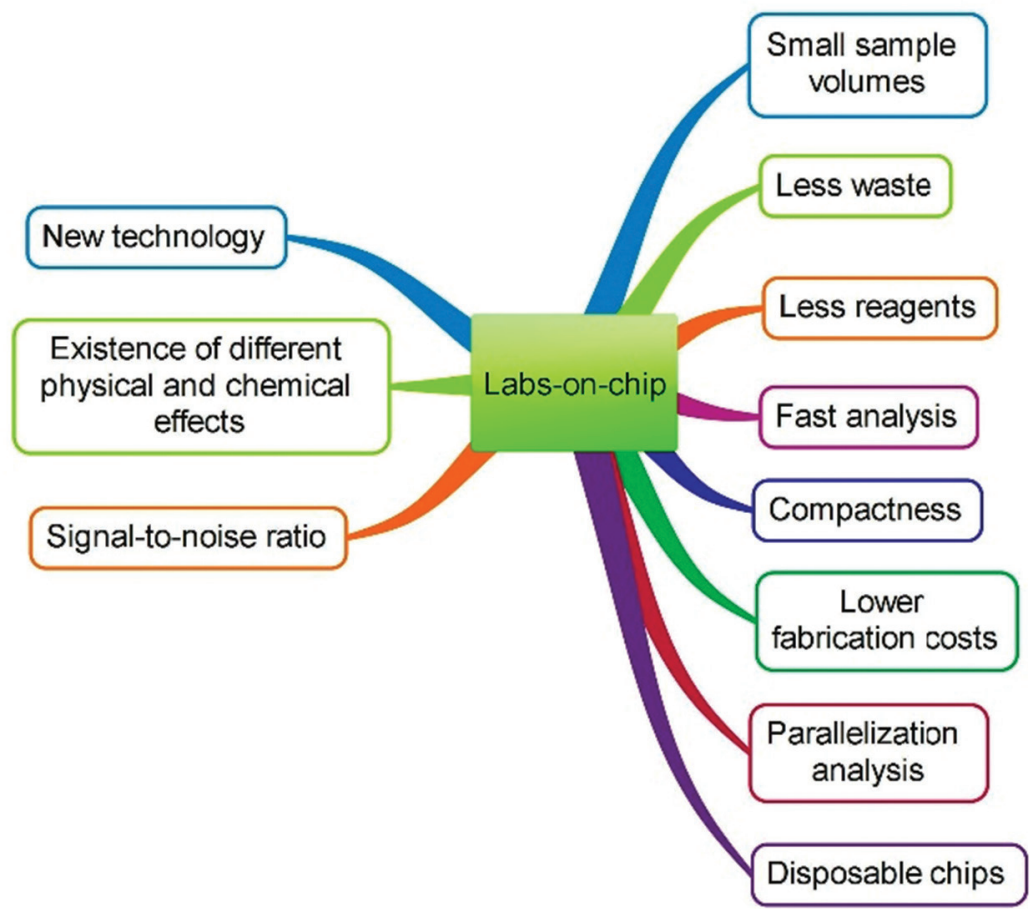

Figure 74. Design considerations and advantages of labs-on-chips compared to conventional technologies.

The first lab-on-chip system was a gas chromatographic microsystem developed in 1975. Developments in this field greatly accelerated about two decades ago. The main commercial applications up to now are in the medical and biological fields. However, applications in several other fields, environment, defense, chemical synthesis etc. are envisaged. ...

There are a lot of advantages to be realized with the development of labs-on-chip devices. Some of them are indicated in figure 74. Also indicated are some of the considerations to be addressed in such research. This is a new technology still in the state of infancy. The appearance of new physical and chemical processes at the nanoscale (see chapter 3 ) necessitates different designs then those existing in conventional laboratories. Furthermore, scaling down detection systems can reduce the signal-to-noise ratio requiring additional development to retain adequate sensitivity. 


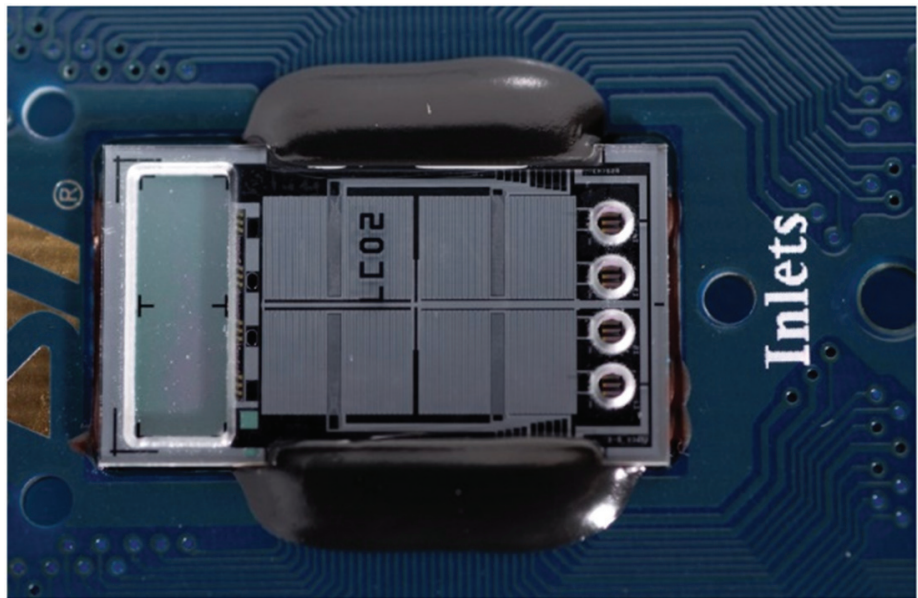

Figure 75. Lab-on-chip dedicated to genetic diagnosis developed by CEA and ST-Microelectronics. Image courtesy of CEA/LETI (France).

An example of lab-on-chip developed by the CEA and ST-Microelectronics is shown in figure 75 . This device is dedicated to genetic analysis. On a single chip it integrates sample preparation using the PCR technique and analysis using hybridization of oligonucleotide probes.

\section{Cells-on-chips}

Cells-on-chips couple living organisms such as cells with semi-conductor technology. Due to receptors on their surfaces cells are able to respond to changes in their environment with high selectivity and sensitivity. In cellson-chips, a change in the cell metabolism or morphology is transduced and analyzed using micro- and nanoelectronics. The cells are the sensing elements and can be used for pathogen detection and toxicology screening, as well as in research to better understand how cells function. Cells-on-chips are organized in arrays, allowing parallel analyses and high speed screening. The key point in cell-on-chip development is to interface living material with inert material. It is for example possible to connect neurons with electronic transistors or to grow cells in microdroplets to create an array of living sensors. These are usually $2 \mathrm{D}$ arrays.

Drug development is an important issue in the health domain. Drugs have to be effective against targeted health disorders but must have minimal negative side effects. Normally a first screening of drug candidates is 
done to find the best candidates. This is done in in vitro experiments. Conventional in vitro platforms try to identify the different signal molecules which are involved in the interaction between a drug and cells but rarely can mimic the cell-to-cell interactions in the body or the extracellular environment. There is now an effort to develop new research platforms based on more complex sets of cells to mimic the $3 \mathrm{D}$ scaffolding of cells to form tissues or organs. Organs-on-chips systems are being developed to mimic the function of livers, hearts, blood vessels, lungs, kidneys, muscles, etc. The liver is particularly interesting for studies of drug metabolism and toxicity. One of the advantages of these developments is to reduce the necessity for animal experimentation.

\section{Summary}

Treating a health problem begins with timely and accurate diagnosis. The less invasive the diagnosis technique, the more palatable it will be for the patient. Therefore, non-invasive imaging and small volume sampling are techniques which have many advantages. Presently in-vitro diagnostic work typically has to be done in specialized laboratories and obtaining results can require hours or days. Required sample sizes can be large. Nanotechnology has an important role to play in the whole diagnostic process. Micro- and nanotechnology allow a natural evolution of conventional diagnosis techniques and can provide rapid and reliable results economically. They also provide exciting new capabilities for research and development of new treatment modalities. Using miniaturized laboratories, such as lab-on-chip systems which are in development and based on microelectronics techniques, can quickly provide a result using a very small amount of sample. Using smaller samples of body fluids or tissues makes the analysis less invasive for the patient. The analysis can be done in a serial manner as it is done today, but also in parallel manner using arrays of miniaturized detectors when possible. Miniaturized analysis systems, including nanoscale components, will provide faster, cheaper results while producing less waste. The final goal is to make them available at the doctor's office. Such low cost high speed analysis systems will soon become routinely available. 


\section{Therapeutics}

Our increasing knowledge of the mechanisms of cell interactions opens the medical field to new treatments which place nanotechnology at the forefront of personalized medicine. Nanotechnology supports the development of more efficient targeted delivery of drugs and offers the possibility of decreasing the drug quantities used while obtaining the same or better results. Sometimes more elaborate therapeutic actions such as surgery and radiation exposure are needed. Nanotechnology can support minimally invasive surgical procedures and can also provide miniaturized implantable devices for long term monitoring and treatment of a patient.

\section{Drug delivery}

Medication constitutes the major portion of medical treatment. Modern pharmaceutics is evolving toward targeted drug delivery, a smart release of drug at the right place, at the right time, and in the right concentration. This can decrease the amount of drug delivered to a patient and provide a greater effectiveness of the treatment. A key point in targeted drug delivery is to minimize drug degradation and loss before it reaches the disease site. In current conventional methods of drug delivery, oral delivery for example, an excess amount of drug is given to the patient in order to get the right dose at the disease site. This excess can have negative side-effect on the patient. This is especially true in cancer chemotherapy where severe side-effects are often observed and need to be counteracted by other medications.

The perfect drug delivery system needs to have the following properties:

- It should protect the drug, allowing it to retain its biological and chemical effectiveness during its transit through the body to the target

- It should release the drug at the proper time and proper rate when the target is reached.

- It should minimize the toxicity and side-effects to healthy parts of the body as it performs its function.

One way to fulfill these conditions is to encapsulate the drug to protect it on its journey and to equip the vehicle carrying the drug with homing characteristics that carry it to the right place and a release mechanism allowing 


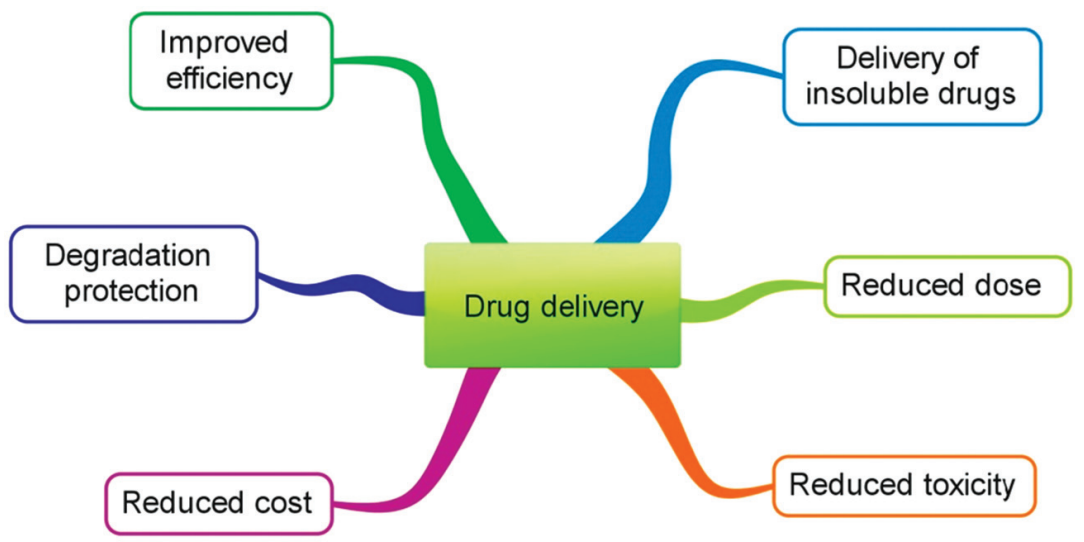

Figure 76. Advantages of nanotechnology in delivery of drugs.

optimal release of the drug on demand. Tailored nanoparticles have the ability to do that and represent a new way to control therapeutic treatments.

The expected benefits of introducing nanotechnology in drug delivery are summarized in figure 76 . Note that encapsulation offers the possibility of delivering insoluble drugs to the appropriate site, an important issue in some cancer chemotherapy treatments.

Nanotechnology also allows detailed control over the rate at which drugs are delivered. The delivery can be passive or active, continuous or pulsed. A passive delivery corresponds to the case where there is a self-degradation of the encapsulating material. In an active delivery the drug is released thanks to an external stimulus. Continuous delivery can be achieved by diffusion of the drug out of a biodegradable polymer, for example. Using an external stimulus such as light, $\mathrm{pH}$, temperature, electric field, etc. to trigger release of the drug allows a pulsed delivery.

\section{Delivery routes}

There are several routes for administration of medication. These are indicated in figure 77. The selection of the delivery method will ordinarily be based on efficiency and effectiveness for the particular disease to be treated.

The most common technique to deliver drugs is oral delivery. Oral medication is convenient in administration, well accepted by the patient and 


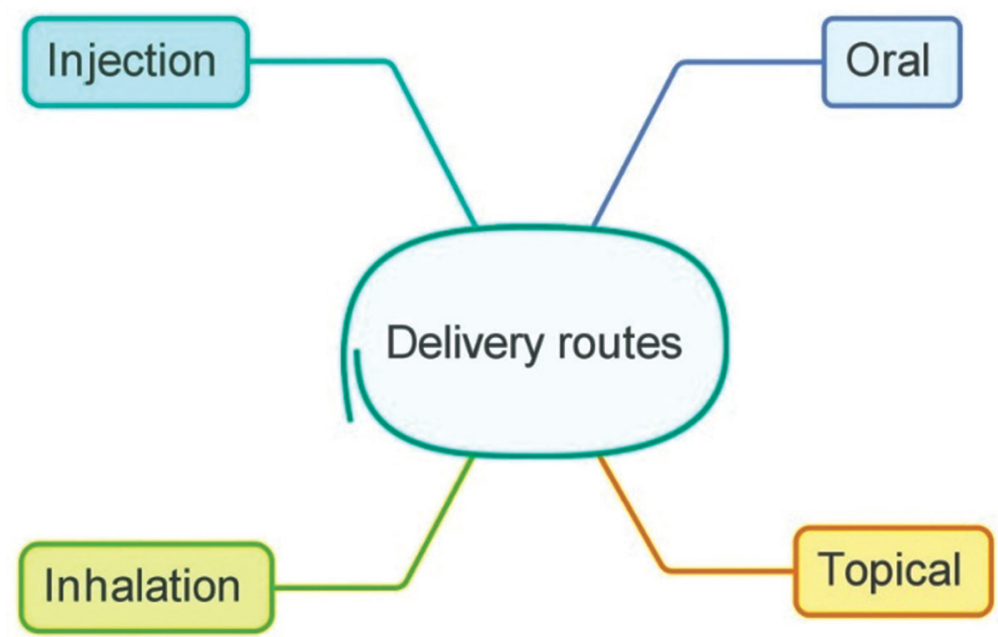

Figure 77. Different routes of administration of medication.

the manufacture of oral medicines is cost-effective. However, part of the drug may not reach the target leading to possible adverse side-effects.

Intravenous, intramuscular or subcutaneous injections are efficient ways to deliver drugs, but administration may be painful and require aseptic conditions for the patient.

Topical delivery consists in delivering the drug on or near the site to be cured, skin or mucous membranes for example.

Inhaled drugs are absorbed quickly and act locally. An inhaler device can be employed to deliver the right dose. Inhalation allows rapid drug delivery to the brain.

\section{Drug carriers}

Drug carriers, typically soluble entities or particulate matter of size less than one micrometer, offer the ability to deliver a drug to its intended target and to release it at that location. Many delivery strategies employ colloidal carriers. Colloidal systems encompass structures such as micelles, liposomes nanoparticles, etc. Micelles are formed with molecules having a hydrophilic (water attracting) head and a hydrophobic (water repelling) tail. Liposomes are vesicles formed with a lipid bilayer (figure 78). The hydrophilic head is directed toward the surrounding solvent (water) and the hydrophobic tail is directed inwards. A micelle can be formed with a variety 


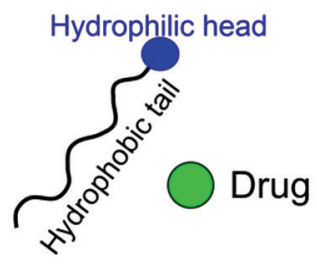

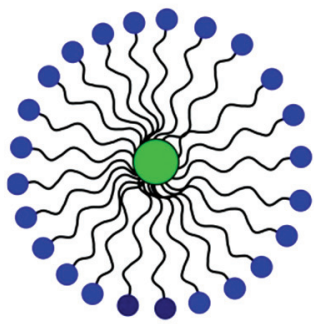

Micelle

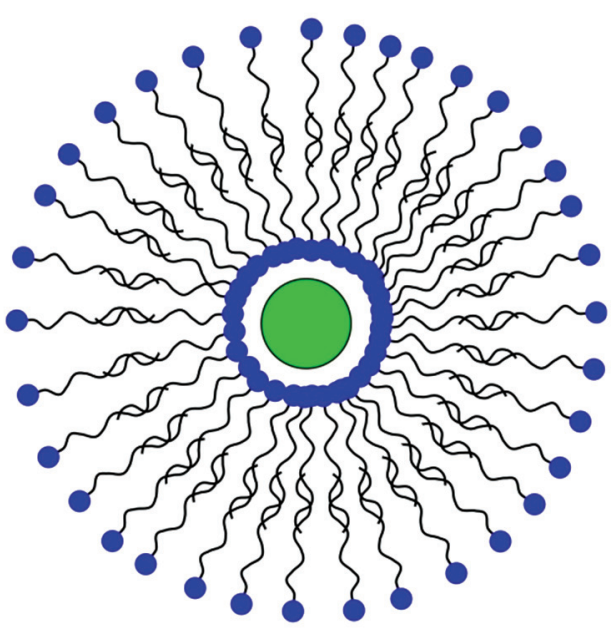

Liposome

Figure 78. Schematic representation of a micelle and a liposome.

of molecules such as fatty acids (soap), phospholipids, detergents, etc. In a micelle, the molecules form a hydrophilic shell and it is possible to put a molecule (drug) inside that shell. Especially interesting is the case in which the drug molecule itself is insoluble in water. The carrier makes it feasible to administer a larger concentration to the patient than is possible in the case of administering the free drug molecule. Furthermore, the shell protects the drug molecule until it is delivered to the right place.

The high selectivity of biological molecules, such as hormones or neurotransmitters, with respect to other molecules, is due to a "key-and-lock" mechanism (figure 79) in which a molecule can bind perfectly to another one. For example, a hormone (the key) binds to a receptor molecule. When this take place at the surface of a target cell, the properties of the cell are modified. Several drugs behave similarly to hormones and produce the same responses when they bind to the receptors. They are called agonists. Examples of agonists are morphine and nicotine. Other drugs (antagonists) behave in exactly the opposite way. When they bind to the receptor they do not produce the same response. Rather they inhibit responses. Examples of antagonists are caffeine, atropine and endocrine disruptors.

A molecular imprinted polymer has properties similar to those of active biological molecules. It behaves as a key to a receptor site molecule. This is schematically illustrated in figure 80 . Because of their similarities 


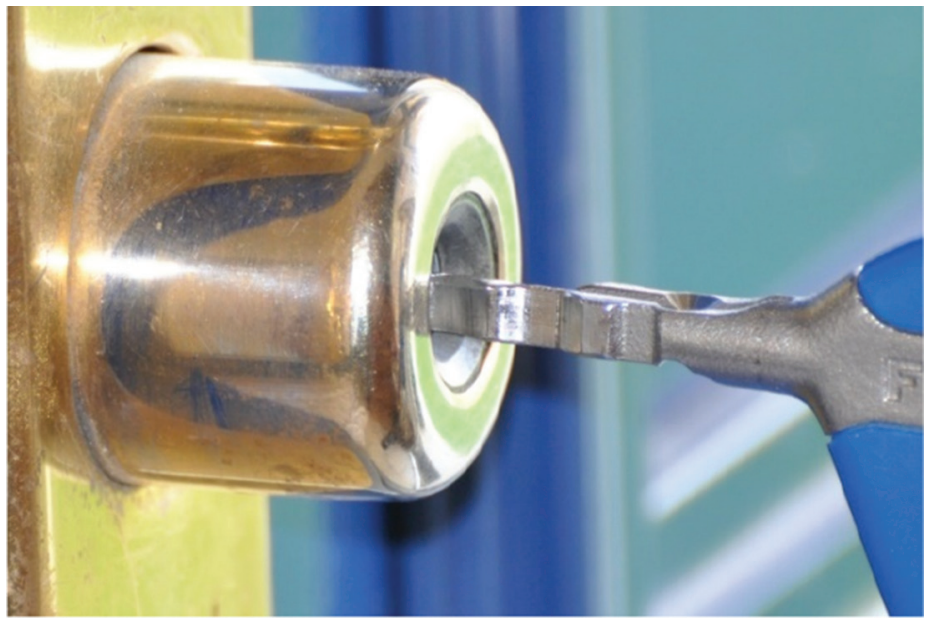

Figure 79. The key-and-lock scheme.
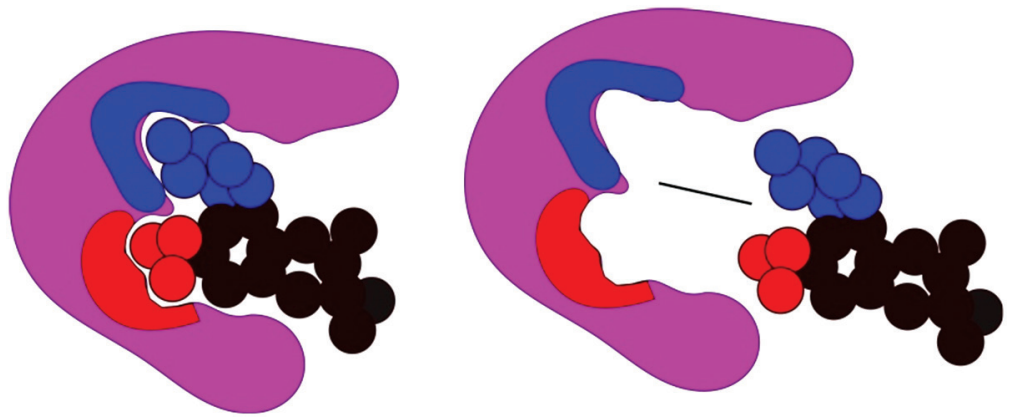

Figure 80 . Interaction of an imprinted polymer with a receptor.

to biological molecules, imprinted polymers have great potential in the field of drug delivery.

\section{Nanoparticles and drug delivery}

Nanoparticles provide several advantages for drug delivery compared to conventional methods. They are stable and can be designed to be highly specific. They can also carry a high dosage of drug while protecting it from outside interactions.

It is possible to graft specific molecules at the nanoparticle surface. These molecules can have different functions. Some molecules can make 


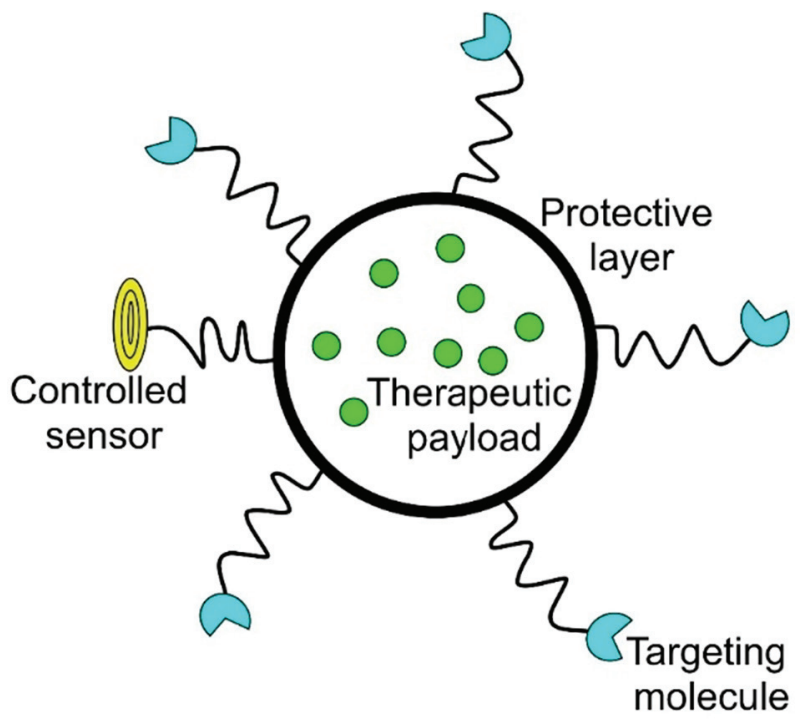

Figure 81. Illustration of a drug delivery nanoparticle. The nanoparticle, which can be a biodegradable polymer for example, encapsulates the drug (green circles) which constitutes the therapeutic payload. The nanoparticle can also contain an imaging contrast agent. The surface of the nanoparticle plays also the role of shield to make the nanoparticle invisible to the immune system. Targeting molecules grafted to the nanoparticle surface guide it and attach it to the cells of interest.

the nanoparticle invisible to the immune system so that they can travel through to the target without being attacked by the immune system. A typical illustration of this concept is shown in figure 81. Since the drug is protected until it reaches the target region, the side effects of the drug are minimized. Other molecules, with the ability to target specific cells, cancer cells, for example, can be attached. After reaching the target, the drug can be released using either passive or active techniques (see above). A schematic illustration of a functionalized nanoparticle is shown in figure 81 .

There are several families of nanoparticles that can be used to improve therapeutic treatments. This is a fast developing area and here we quote a few examples which illustrate the possibilities.

Drugs are often insoluble into water. Higher quantities than necessary are administrated to the patient to be sure that the right dose reaches the target region. This can lead to side effects because part of the drug is lost during the journey in the body of the patient. Encapsulating the drug into nanoparticles soluble in water can overcome this drawback. They are several such nanoparticle based techniques. A colloidal dispersion of nanoparticles is used for intravenous delivery of insoluble molecules. This allows more efficient delivery of the drug payload. 
Colloidal gold has been known since ancient times. Gold nanoparticles are easily manufactured and functionalized and have good biocompatibility. They are used in the treatment of rheumatoid arthritis and sclerosis. Small size gold nanoparticles, typically with diameters smaller than 5onm, have the ability to cross the brain-blood barrier. This is an interesting property which allows transfer of drugs from the blood to the brain.

Protein nanoparticles are often used for drug delivery. Albumin nanoparticles are used to deliver paclitaxel, a cancer drug, isolated from the bark of the Pacific yew tree. Paclitaxel is a highly toxic hydrophobic product. It is used in chemotherapy to treat lung, breast and ovarian cancers. Paclitaxel has many possible side effects such as a decrease in white blood cells, red cells and platelets; hair loss, inflammation of the mucous membranes, damage of the nerves, water retention, skin reactions, nail alteration...The cancer drug Abraxane is a solvent-free formulation of the paclitaxel. Binding Paclitaxel to albumin nanoparticles that will, in turn, bind to the tumor tissue reduces the toxic side effects and $50 \%$ more drug can be delivered at the right place.

Chitosan is a polysaccharide obtained from crustaceans such as shrimps. It has several applications but an interesting one is that it has the ability to clot blood. Nanoparticles of chitosan can be included in bandage and hemostatic agents. Chitosan has also antibacterial properties.

Another helpful application of nanoparticles is thermotherapy. By time-resolved infrared imaging it is possible to know the exact location of a tumor in the body by locating nanoparticles, attached to cancer cells. After this is done, it is possible to heat this specific region by laser irradiation and kill the cancer cells. Another advantage of time-resolved infrared imaging is that it can find metastasis cells located far from the initial tumor allowing the ability of an early treatment.

As a last example, consider carbon nanotubes. Either open or closed at one end (nanohorns). they can be used to encapsulate molecules, proteins and nucleotides. Their surfaces can also be functionalized. Carbon nanotubes have the ability to penetrate into cells without harming them, thanks to their very small size. Carbon nanotubes are particularly useful to deliver insoluble and toxic drugs because they are encapsulated and protected.

\section{Summary}

Drug delivery is an essential tool in health treatment. The goal is to deliver the right quantity at the right place and at the right time in order to 
maximize efficiency and minimize any side-effects. Nanoparticles have many advantages for drug delivery compared to conventional techniques. Drugs can be attached to nanoparticles that are eventually functionalized to go at the right place in the patient's body. Drug molecules can be also encapsulated within a functionalized nanoparticle that guides and releases them at the right place while protecting them during the travel in the body of the patient. With these new techniques, the trend towards dedicated treatments and personalized medicine is greatly accelerated. 


\section{Regenerative medicine}

In Figure 67 we noted the three main areas to be addressed in health care: diagnostics, therapeutics and restorative or regenerative medicine The two first steps have been addressed in the two preceding chapters. The goal of regenerative medicine, the third main area of health care, is to repair diseased parts of the body. Some people are born with serious deficiencies. Accidents can lead to serious damage of the body: Some parts are destroyed or seriously injured. The aging of populations in many countries also leads to an increasing demand for regenerative medicine.

One opportunity offered by nanotechnology is that it is possible to manufacture objects with properties similar to those of naturally occurring nanoscale structures. This opens the possibility to repair damage to cells or tissues in a way similar to the way biological systems do it, or to manufacture devices with the ability to correct functional deficiencies in the human body.

\section{Biomaterials}

An important requirement for biomaterials is that they be accepted by the surrounding tissues of the patient and remain biologically inert. Biomaterials were already used almost a half a century ago to replace some limited parts of the body. In the late 1980s, bioactive components with a controlled action on the body of the patient were used in the orthopedic and dental domains. Absorbable materials were also developed at that time. A more contemporary goal in this area is the development of biomaterials with regenerative properties providing self-healing capabilities to skin, bones, cartilage and tissue.

Presently, damaged tissues or organs are often replaced by artificial substitutes implanted in the body (hip replacements, coronary artery bypass grafting, coronary stents...). Nanocoatings can improve the surface properties, durability and biocompatibility of such devices. Nanopolymers are used to coat devices in contact with blood, such as catheters to prevent clot formation. Nanofiber membranes can be covered with antibiotics, painkillers or other specific drugs before being applied to diseased or damaged tissues. 
Regenerative engineering is based on development of absorbable scaffolds with appropriate cells capable of regenerating the damaged or diseased tissue. it is possible to produce regeneration messengers for monitoring the tissue reconstruction. Nanostructures could be used to manufacture temporary, biodegradable materials which do not need to be surgically removed after they have done their job. Within the current decade it is expected that the ability to control the growth of ${ }_{3} \mathrm{D}$ scaffolds will be developed.

Living systems are extremely efficient in many respects. Understanding their function can be of great help in regenerative medicine. Biomimetics is the study of the formation, structure, or function of biologically produced substances for the purpose of creating artificial mechanisms which mimic natural ones. We have already discussed some important natural mechanisms in chapter 5 . The physics and chemistry on which they are based can be adapted to development of artificial devices implanted in the body. An important point is to improve as much as possible the longevity, the biocompatibility and the life time of implanted devices. Bionanomaterials can play a key role in this respect. Bioactive coatings can produce better interfaces between artificial implants and the neighboring tissues. Nanocoating can also help to prevent immune rejection mechanics by building a barrier at the interface between tissues. Smart materials which respond to external stimuli such as temperature, $\mathrm{pH}$, electrical signals... can be manufactured in such a way that they respond to the environment in which they are located.

\section{Cell therapy}

Injured tissues heal themselves more or less rapidly depending on their nature. Healing can be rather fast for blood or epidermal injuries but is much slower for bones or cartilage. Cell therapy offers large opportunities in regenerative medicine.

The communication between cells is based on direct cell-to-cell signaling and on the interaction with diffusible biomolecules. This communication process is essential in healing mechanisms. Indeed, a single cell must know what happens in its environment and behave accordingly. Investigations of natural healing mechanisms and identification of the different cell signals present in the communication between cells can be made using different methods. 
Nanoscience can help to understand the complex communication process between cells. In the future it is expected that nanotechnology can replace part of this communication process with smart nanodevices delivering biomolecules (proteins, genes...) at controlled rates to activate cells to produce the growth factors necessary to initiate the healing mechanism of the tissue. Stem cells have an unparalleled ability to regenerate different kind of tissues and nanotechnology can be used to provide delivery vehicles to bring them to the right location.

\section{Implants}

Implants are often needed to replace important parts of the human body. There are two main issues with implants. The first one is that they can be rejected by the body. The second is that they can be worn out and require replacement. Artificial hips, for example, typically need to be replaced after 10-15 years. Nanocoatings can be designed to improve biocompatibility and increase the mechanical resistance to wear. They can also be employed to prevent adherence of biological material; an important property for synthetic vascular grafts and other artificial materials. Vascular implants and stents are extensively used to treat some cardiovascular diseases. Additional capabilities such as protection from bacterial or fungal infections can be incorporated. Nanomaterials can provide lighter weight implants with longer lifetimes than those of conventional implants.

Another important issue for implants requiring electricity to function is provision of an appropriate energy source. Lithium-ion batteries can have lifetimes of the order of 7-10 years. Induction charging is an interesting possibility to recharge the battery without any surgical interaction in the body of the patient.

For repair of bones and teeth, it is necessary to have a good osseointegration of the implant. This depends very much on the interface between the implant and the surrounding bone. It turns out that the larger the surface of the interface, the faster is the healing process. Titanium, a metal widely used in bone implants, has an improved osseointegration in bone repairs when the surface of the implant is nanostructured. Nanotechnology will play a greater role in the future.

Methods to repair damaged cartilage, a problem for a large number of the aging, are also being developed.,. In vitro cartilage tissue regeneration techniques are being explored. New cells generated in this fashion can be 
transplanted into the body. Other areas such as tracheal, esophageal and bladder regeneration can also benefit from such in vitro techniques.

Neural implants present a particularly difficult problem. Silicon nanowires have the ability to detect a larger number of signals coming from the neurons than do conventional electrodes. Because of their good electrical conductivity, carbon nanotubes are being investigated as replacements for axons. Nanodevices are also being developed to electrically stimulate some specific part of the brain for the treatment of neurodegenerative diseases such as Parkinson's disease.

Vision and hearing are two critical senses and progress is currently made to develop retinal and cochlear implants. Nanotechnology allows development of smaller and more powerful devices to restore vision and hearing.

According to the United Nations (UN) The share of elderly people,defined for this purpose to be people older than 60 years, among the global population has increased from $9.2 \%$ in 1990 to $11.7 \%$ in 2013 . The UN foresees this proportion of the world population to reach $21 \%$ by 2050 . This increase results from a decreasing mortality and a decline of fertility. There are presently about 45 million people in the world suffering from blindness, some since birth, others as a result of accidents or disease. This number is increasing as the population ages.

Some of the diseases leading to blindness or severe visual loss cannot currently be prevented or cured. Retinis pigmentosa, an inherited disorder affecting about 1 million of people in the world is one of these. Artificial vision using prosthetic devices is then the unique current solution of the problem. Artificial vision is based on an electric device implanted in the eye to simulate nerve tissues. Electrical signals containing the visual information are transmitted to the brain creating virtual points of light which can create an image for the patient. The technologies under development can be classified according to the coupling between the electrodes of the device and the tissues (http://www.nidek-intl.com/ visual_prosthesis).

A retinal implant (called also artificial retina) stimulates the retina with electrodes. The retina can be stimulated by three major methods: from the top side (epiretinal implant) by implanting an electrode array on the retinal tissue, by implanting an electrode array under the retina (subretinal implant) or the suprachoroidal transretinal stimulation where a stimulation electrode is placed inside the sclera which is the tissue covering the outermost part of the eye and where the return electrode is located in the intravitreous part of the eye.

This is summarized in figure 82 . 


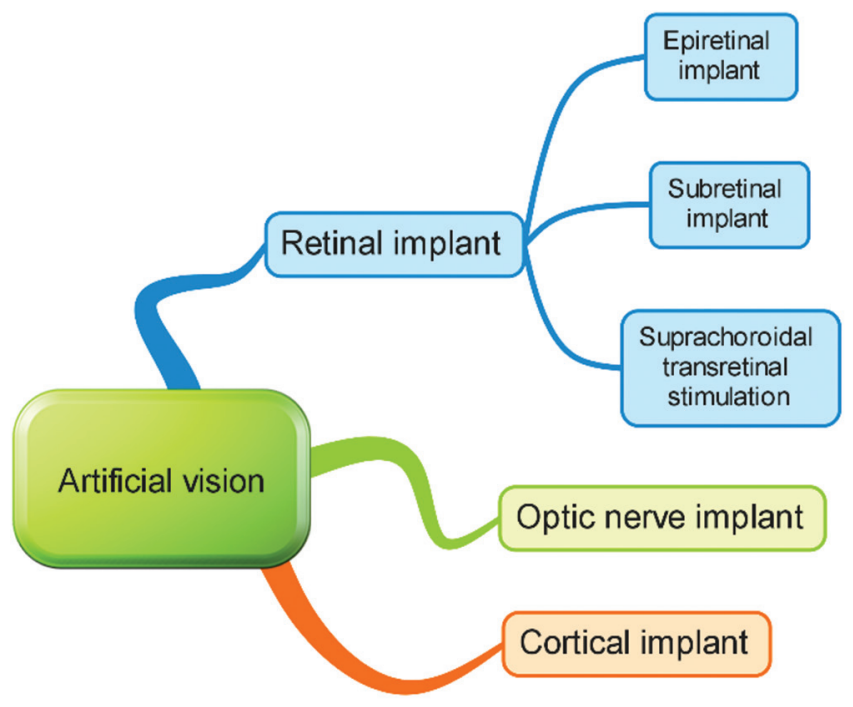

Figure 82. Main routes for enabling artificial vision.

A less advanced technology is a nerve implant where a cuff electrode array is wrapped around the optical nerve. A camera provides an image which is transformed into electric signals and sent to the nerve electrode array to stimulate the optical nerve. Training is necessary and this system requires a lot of software.

Cortical implants are located inside the brain to stimulate directly the visual cortex after transforming the image of a camera into electrical signals. A $5 \times 5$ signal array (25 pixels) provides the ability to read some letters and a $10 \times 10$ array (100 pixels) provides the ability to distinguish some shapes. A minimum resolution of a $32 \times 32$ array (1024 pixels) is necessary to provide a person an image allowing that person to function with some freedom. A retinal prosthesis project with 1500 electrodes has been developed recently in Tübingen, Germany. This is much larger than most of the projects which have less than 100 electrodes.

\section{Dentistry}

The main goal of dentistry is to maintain the health of oral tissues. Nanotechnology can be applied in all areas of dental care, prevention, diagnosis and treatment. 
Periodontal diseases have heavy consequences on the oral cavity: demineralization of the enamel and the dentin, degeneration of the soft tissue and the periodontal ligament, etc. The regeneration of lost tissues is therefore an important issue. Three main strategies are carried out in this respect: bone grafting, bone substitutes and guided tissue regeneration. Nanotechnology can be useful in the last two strategies. Nanohydroxyapatite particles with a size of $18 \mathrm{~nm}$ can be used as bone substitute or in the remineralization of teeth following exposure to carbonated beverages. Nanotubes and carbon nanofibers can also speed up the process of bone regeneration.

Titanium is a metal often used in orthopedics and dentistry thanks to its high fracture resistance, ductility and lightweight. Its disadvantage is that does not enhance cell adhesion and osteoblasts development. Coating the implant with nanofibers or nanostructures can mitigate this problem.

Using nanoparticles with antimicrobial properties allows a decrease in the number of long-term antibiotic treatments with the advantage of reducing the extension of antibiotic resistance. For example, metal nanoparticles such as silver nanoparticles are efficient against Gram negative and anaerobic bacteria. The efficiency depends on several factors such as the concentration of nanoparticles (a too low concentration is inefficient and may induce bacterial resistance), on their sizes (smaller nanoparticles are more efficient than larger sizes particles), on their geometries. Figure 83 summarizes the kind of nanoparticles with antimicrobial activity which can be used in dentistry ${ }^{10}$.

Nanofiber scaffolds can be used to deliver hormones and cells without triggering an immune response in the body when they dissolve. In dentistry, these scaffolds are used for regeneration of oral and dental tissues. (alveolar bone, periodontal ligament, dental pulp, enamel).

Nanotechnology can also be used in drugs delivery systems. The drugs which are encapsulated into nanoparticles are protected during delivery preventing toxic side-effects Nanocoatings, such as those made of silica nanoparticles can be used for the delivery of antibacterial drugs. The delivery can also be triggered by an external signal, such as laser irradiation or using magnetic interactions. Delivery of various agents (antibodies, proteins, cell-targeting drugs) can be made with loaded nanoshells. Nanoshells are nanoparticles with a dielectric core (silica for example) and a thin metal coating (gold for example). Nanoshells can also be used alone because they are sensitive to infrared radiation. Infrared radiation is absorbed by the nanoshell and its temperature increase gives the ability to destroy bacteria, cancer cells etc. 


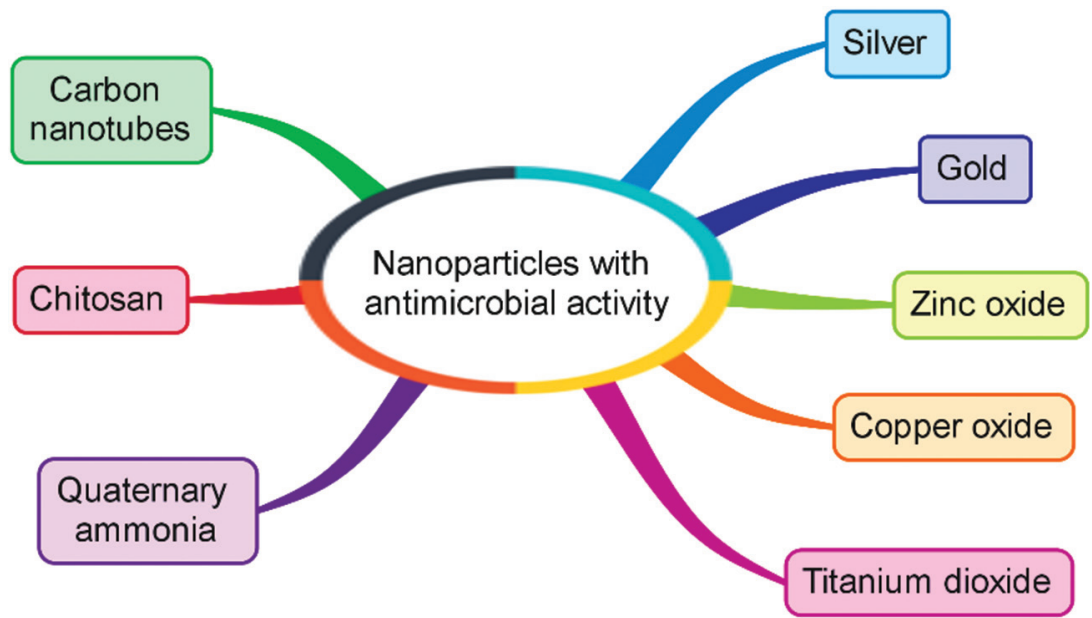

Figure 83. Nanoparticles with antimicrobial activity. Chitosan is a polysaccharide with high biocompatibility and coagulative properties obtained from shrimp and crustacean shells.

Quantum dots nanoparticles of lead sulfide, zinc sulfide, indium sulfide are semiconductors. Quantum dots are also useful in dentistry because they can absorb and reemit light at a definite wavelength which depends on the size of the quantum dot. They can be coated with molecules which have a tendency to attach to cancer cells. The size of the quantum dot can be adjusted so that they absorb and emit in the UV region and can be used in the detection of oral cancers.

\section{Nanosurgery}

The modern trend in surgery is to be minimally invasive lessening patient trauma and promoting prompter patient recovery.

Crystalline or polycrystalline silicon can be used to make surgical blades with cutting edges between 5 and $500 \mathrm{~nm}$. This is useful in eye surgery.

Nanoneedles with a diameter between 200 and $300 \mathrm{~nm}$ can penetrate into living cells without harming them. They allow performance of cell surgery but can also be used to deliver specific drugs to the cell.

Biological molecules inside a cell can be manipulated with nanotweezers with a thickness below $50 \mathrm{~nm}$. This opens the way to perform DNA manipulations and perform physical measurements.

Femtosecond laser surgery is a technique with a great potential in this regard. It uses a high intensity light beam in the near infrared region (740 and 
$800 \mathrm{~nm}$ wavelength). It has the ability to operate on a volume smaller than a femtoliter $\left(10^{-15}\right.$ liters). With this technique, a cut size of $110 \mathrm{~nm}$ has been achieved allowing chromosome dissection within a single living cell. Future applications of this technique in eye surgery, neurosurgery, gene therapy, etc. will have great impact.

\section{Summary}

Some people are born with severe medical problems. Others contract debilitating diseases later in life. Some people get seriously injured in accidents. The population is ageing. For all these reasons there is an increasing demand for regenerative medicine. Biomaterials and implants are one route to regenerative medicine. These areas have been developed for a long time but nanotechnology can support the maintenance of healthy tissue and progress in regenerative medicine, opening possibilities that have not previously existed. Cell therapy offers a powerful technique for self-healing of biological materials such as bones, tissues. Nanosurgery is developing techniques to work at the cell level with the possibility of modifying genes. 


\section{The Food Chain}

The availability, quality and safety of food is a paramount concern for any society. The main concern, at the global level, is an adequate food supply for the planet's inhabitants. The rapid growth of the world's population, an increase from 1 billion in the 1800 s to more than 7 billion today, spurred important changes in the food domain. The introduction of fertilizers, pesticides and insecticides played a major role in allowing the world to respond to this change. Although the role of these products and their use is not without controversy, without them a large part of the world population would starve to death.

\section{Feeding people}

While the human domestication of plants and animals occurred independently in many places at different times, the first extensive development of agriculture appears to have occurred at the end of the Ice Age (about 12,000 years ago). With this development came a necessity to manage the food supply. Although the cooking of raw food, introduced, about 300,000 years ago killed dangerous bacteria, it was necessary to have techniques to preserve and store food to be consumed at later times. New methods such as salting, drying and smoking were developed for that purpose. In the $19^{\text {th }}$ century, new technologies of food processing and storage were introduced when the industrial processing of food began. Canning, freezing, pasteurization, sterilization were introduced. While these processes increased the safety of the food supply, people have often been resistant to changes in food processing. For example, the mandatory pasteurization of milk in the United Kingdom in the 1930s faced a decade of strong resistance despite the fact that this method prevented thousands of deaths from bovine tuberculosis. The question of risk $v s$ reward is often paramount when any new technology appears.

Figure 84 shows different parts of the food domain that can be impacted by nanotechnology. All these parts can benefit significantly from nanotechnology. However the introduction of such techniques must be based on sound research and carried out with appropriate scrutiny and controls.

The different steps of the food supply chain, from "farm to fork", are indicated in figure 85. For each of these steps nanotechnology can provide improvements to existing techniques or bring new solutions. 


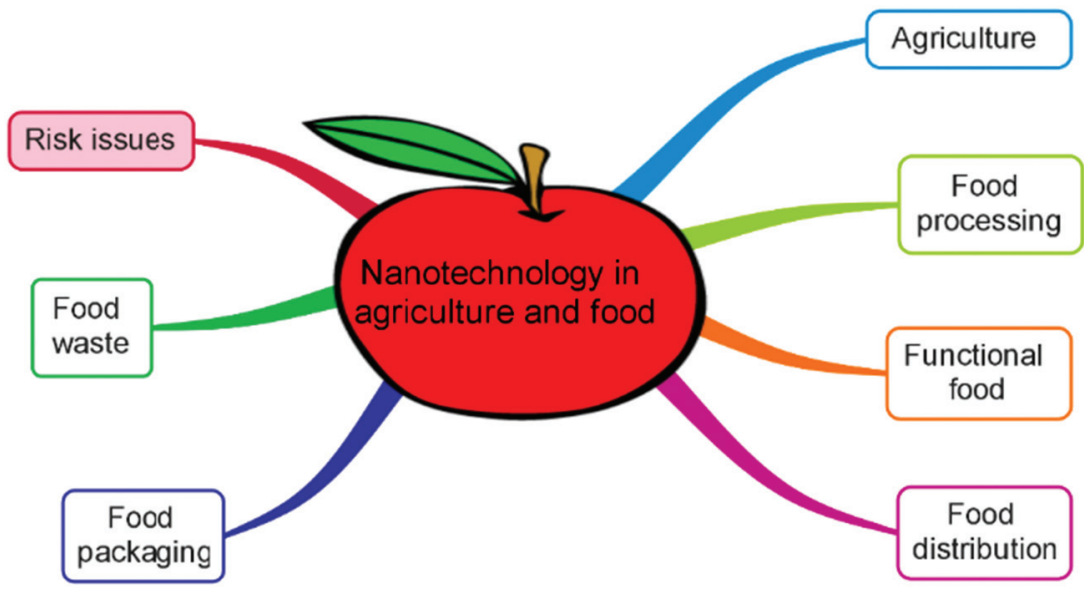

Figure 84 . Some parts of the agriculture and food domain which can be impacted by nanotechnology.

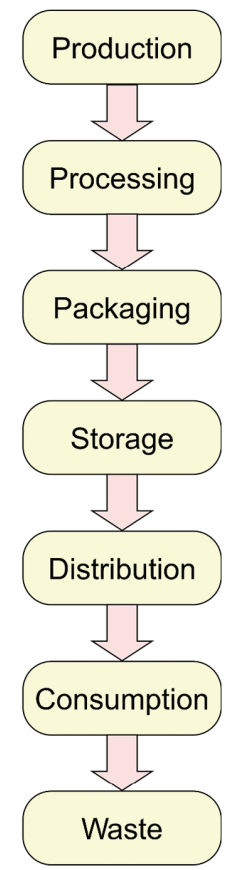

Figure 85. Different steps from «farm to fork» . 


\section{Agricultural Production}

Most of the food consumed in the world is produced by planned agriculture. Agricultural techniques are continuously improving thanks to new technologies appearing on the market. The challenge of modern agriculture is to meet global food requirements while using available agricultural land as efficiently as possible, to reduce the need for resources such as fresh water and to minimize the use of agricultural chemicals (pesticides and fertilizers) that have adverse effects on the environment. It is not surprising that nanotechnology has also applications in agriculture.

Nanoparticles offer the potential to deliver nutrients, pesticides and fertilizers in a more controlled and efficient manner. A controlled release of fertilizers or pesticides decreases the amount of chemical product required because of increased efficiency. One consequence of that is a reduction of ground water pollution. Active nanoparticles with nanosized additives can also be used to remove microbial and chemical soil contaminants present because of past treatments. In developed countries, there is a trend towards such "precision agriculture".

A better delivery technique requires a close monitoring of the external conditions. To be as efficient as possible, the chemicals have to be delivered to the right place at the right time, and in the right amount. Nanosensors, and especially biosensors, and sensors including nanoelectronics are able to do that. In addition to monitoring the external environmental variables such as the ambient temperature or the level of water, they are also useful to perform analytical measurements to get the physical, chemical and biological properties of the soil and evaluate its fertility. Sensors can also be used to monitor plant and animal health and growth. Disease and pest control in crop plant and domesticated animals are important issues. The decrease of the cost of sensors, thanks to new nanoelectronic and microelectronic techniques will accelerate the use of such methods.

Agriculture can also be used to produce nanomaterials, both natural nanomaterials that can be grown and harvested, or manufactured nanomaterials which use plant material as raw starting material.

As with any new technology, care must be taken to avoid possible negative impacts. Each new technique has to be carefully investigated to evaluate effects on human health. For example, while it is known that carbon nanotubes accelerate the absorption of water into the soil the possibility that significant amounts might enter the food chain and the consequences of that must be carefully evaluated. 


\section{Food processing}

Chemical and biological interactions at the nanoscale level or below are present in all food products. They are responsible for the taste, the texture, the viscosity, etc. of the product. In the past, the production and processing of food was mainly done empirically. Our modern understanding of the microscopic interactions allows us to elaborate the properties of a food product on demand.

A large number of natural foods contain nanoscale components. They have been eaten safely for centuries. Dairy technology has existed for a long time and it is well known that microsized or nanosized structures are present in milk. Homogenized milk has a nanostructure of droplets with a size of about $100 \mathrm{~nm}$. Microsized and nanosized structures (casein micelles, fat globules and whey proteins) are the basic ingredients in dairy product emulsions (butter), foams (ice cream or whipped cream), liquids (milk), plastic solids (cheese) and gel networks (yogurt).

In modern times proteins, starches and fats are often modified and transformed during food processing, introducing nanostructures to improve the properties quality and safety. At the global level, there are many that incorporate a nanoproduct in one form or another during manufacturing. Nanomaterials have been used for a long time in the form of additives to make cooking processes easier. This is the case of nanoparticles of silica that are used as anti-caking agent in powders such as flour. Nanoparticles are also used sometimes to enhance taste. This is the case of some milkshakes that use nanosized silica-base compounds.

Nanostructures present in food may have four origins (figure 86). They can be natural substances or substances included while processing it or preparing it for consumption. Examples of such nanostructures that can be included are sugar molecules, micelles, fat, nanoscale proteins. The processing can also generate nanoscale particles, either during a conventional process or deliberately. Engineered nanosubstances may be introduced to provide new properties.

Engineered nanomaterials found in food products can be inorganic, surface functionalized and organic nanomaterials. Surface functionalized nanomaterials bring antimicrobial activity. Inorganic nanomaterials have applications in food, food additives, packaging and storage. They include silver, iron, calcium, magnesium, selenium, silicates, titanium dioxide, etc. Titanium dioxide nanoparticles are for example often used as a color or flow agent in foods. Nanocatalysts based on nanostructured metal nanoparticles are very efficient to accelerate reactions and control the paths of 


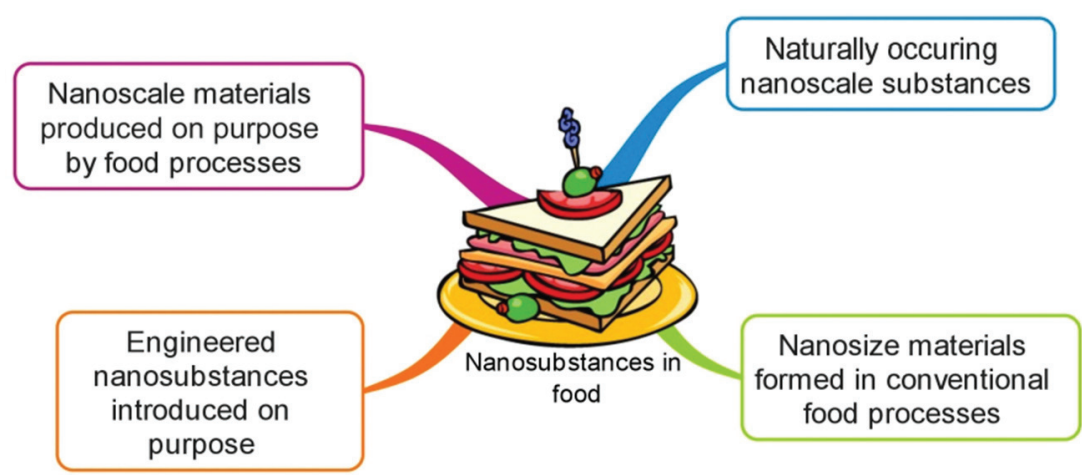

Figure 86. Different kinds of nanosubstances present in food.

some reactions used in the food domain. An example is the reduction of trans-fatty acids during the hydration of fats.

Modern life favors a sedentary lifestyle and several health problems such as cardiovascular diseases, obesity, diabetes, etc. are on the rise. Engineered nanoparticles can be used for their preventive healthcare properties. For example, it is possible to use nanotechnology to manufacture a foodstuff with a low fat content but with additives that mitigate any degradation of taste and the texture. Nano-ingredients are often better absorbed by the body than macroscopic ingredients. Many vitamins and other substances that are insoluble in water are more easily absorbed when they are encapsulated inside nanoparticles. Nanoencapsulation can utilize nanoemulsions or nanoparticles. This technique protects the nutrients from environmental conditions and improves the uptake of nutrients in the human body. Compared to a century ago, the food available to the consumer is now rather sophisticated.

The ability to tailor new food products with nano-ingredients such as vitamins, anticarcinogens, etc. provides the opportunity to prevent or mitigate a predisposition to allergies, diseases or disorders in the human body. Figure 87 indicates examples of nanomaterials for nutrient delivery.

There is a great deal of current research addressing the possibility of using nanotechnology to enhance food. By introducing specific nanoparticles, it is possible to give to the food new properties. The advantages of positive additional features obtained by adding artificial nanostructures should always be balanced against the potential risk of doing so. 


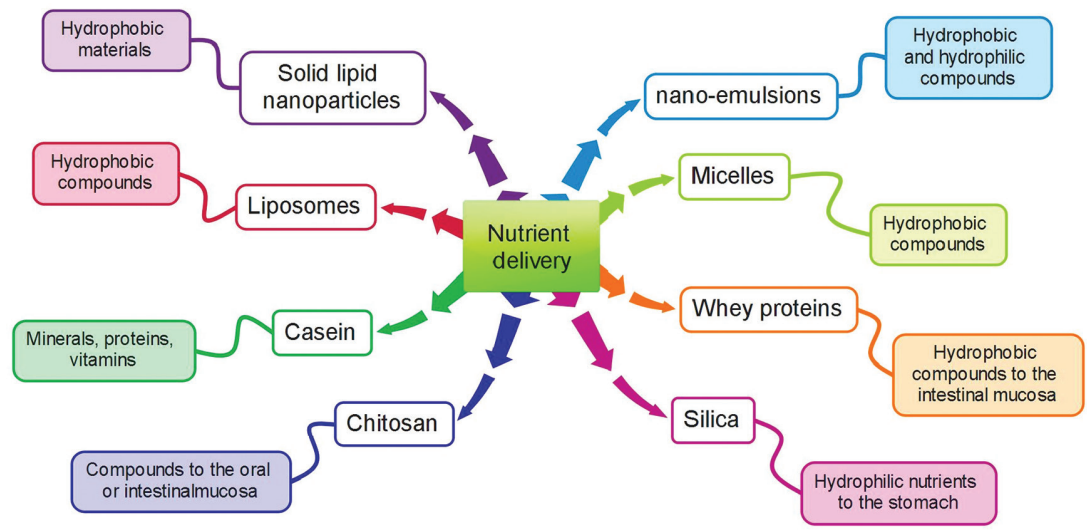

Figure 87. Nanomaterials for nutrient delivery. Information taken from the European ObservatoryNano project.

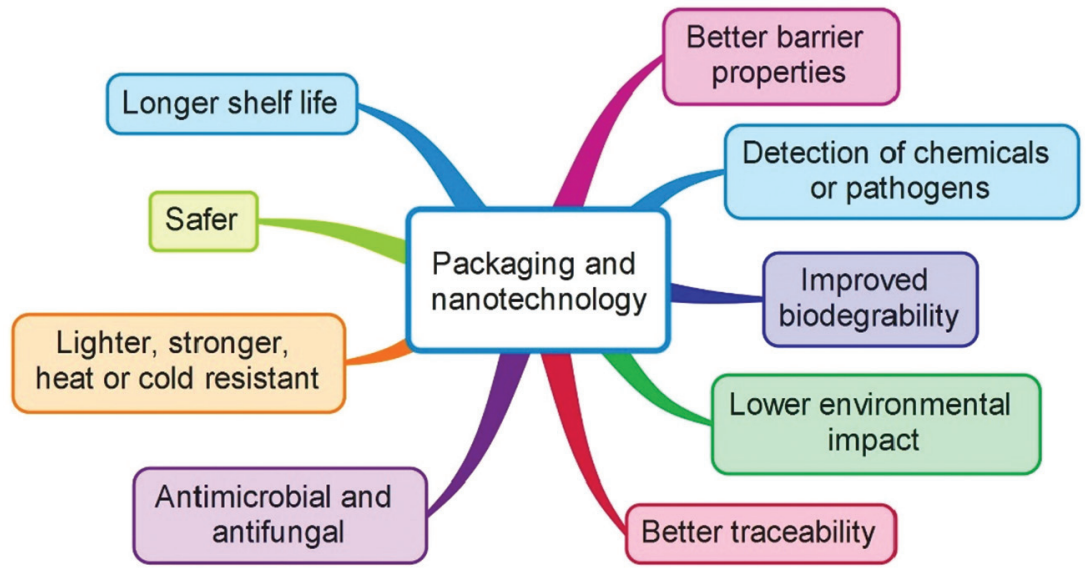

Figure 88. Potential improvements offered by nanotechnology in packaging.

\section{Packaging}

A main current area of application of nanotechnology in the food sector is packaging (figure 88). Preserving and protecting food is an important issue. Packaging is a key step in handling and commercializing food products. Maintaining a high level of quality and safety of the product as it is transported from the source to seller to customer is essential.

Metal and metal oxide engineered nanoparticles have major applications in food packaging. Nanosilver, for example, has antimicrobial and anti-odorant properties. Nanoselenium is used as an additive to green tea 


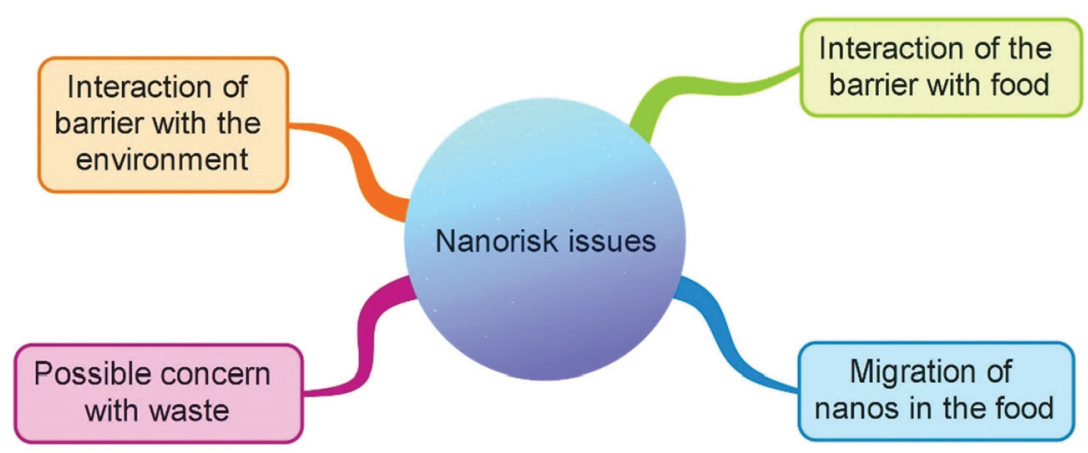

Figure 89. Use of nanotechnology in packaging may have some risks, as indicated.

products because of the health benefits of selenium and allows an enhanced uptake in this form. Nanocalcium and nanomagnesium salts are also available as health supplements, as is nano-iron which, in addition of being a health supplement, can also be used to decontaminate water.

Surface functionalized nanomaterials can add new functionalities such as absorbing oxygen to prevent oxidation or providing antimicrobial activity. Functionalized nano-clays are used to develop materials that have enhanced gas-barrier properties. Nanoencapsulation of bioactive components that are delivered at the right place with high efficiency are emerging applications in the food industry domain.

Biosensors are important tools for identification of bacteria and monitoring food quality. Smart packaging is a packaging containing nanosensors and antimicrobial activators.

There are also potential risks in using nanoscale materials in packaging. These risks have to be carefully evaluated to prevent any health problems to the consumer. The main risk issues are shown in figure 89 . There is always an interaction between the barrier and the food that is contained. There are also interactions between the packaging and the environment.

\section{Transportation}

Food is not always produced close to the place where it is consumed. Transportation is required to bring food to the people. Transportation generates pollutants and emits $\mathrm{CO}_{2}$. Reducing transportation volumes and costs has a number of advantages. Some foods can be dried before they are transported and distributed. The development of dry particulates of food that 
can be rapidly, easily and safely rehydrated while keeping the initial taste and nutritional properties is one method which offers this advantage. Nanotechnology may provide enhancement of industrial scale processes for drying and rehydration of food.

\section{Summary}

Nanotechnology is already widely used in the food industry with various applications from "farm to fork". Nanostructured food ingredients can tailor the taste and texture of food products. Nanosized materials can increase the uptake and absorption of several products such as vitamins or antioxidants compared to conventional uptake of macroscopic equivalents. Nanotechnology in packaging can provide new level of protection to our food supply. Nanotechnology can also be present in sensors monitoring food processes and packaging. Micro and nanosensors are welcome innovations to monitor food production. They can detect bacteria and pathogens in a much shorter time than conventional methods and at a reduced cost. For that reason, they can be used on line during food production.

Thanks to their very small size, nanoparticles and nanosized objects can penetrate easily into all parts of the human body. Any serious health incident in this field could severely affect the evolution of nanotechnology in the food domain. Except for sensors, any broader development of the nanotechnology in the food domain depends very much on the desirability of the enhancement envisaged and the confidence and trust that society attaches to these innovations. 


\section{From microelectronics to nanoelectronics}

By controlling the flow of electrons, electronic devices allow us to process, store and transmit information. The technological advances which created the possibility to manufacture cheap integrated circuits containing very large numbers of components produced a microelectronics revolution. The first such integrated circuits were developed by Jack Kilby at Texas instruments, and Robert Noyce and Jean Hoemi at Fairchild in 1958-59. The integrated circuit of J.Kilby was very simple: one transistor, one resistor and one capacitor, but all on the same germanium crystal with gold wire interconnections. The integrated circuit manufactured by R.Noyce and J.Home had interconnections deposited on the surface and was manufactured using a planar process.

The first commercial integrated circuit became available in 1961. Today more than a billion transistors can be found in some microprocessors. For example, the Ivy Bridge microprocessor manufactured by INTEL contains 1.4 billion transistors on a surface of $160 \mathrm{~mm}^{2}$. This is already in the domain of nanoelectronics since these transistors are manufactured with a lithographic technology having a resolution of $22 \mathrm{~nm}$. To realize how small such a detail of each transistor is, remember that a red blood cell measures about 7,000 $\mathrm{nm}$ and the AIDs virus has a size of $100 \mathrm{~nm}$. This is, however, larger than the size of a buckyball $(1 \mathrm{~nm})$. Thanks to the semiconducting properties of silicon and to the exceptional physical properties of silicon dioxide, silicon technology is currently the dominant technology in microelectronics. Integrated circuits are made on slices of silicon called wafers. One example of a silicon wafer containing integrated circuits is shown in figure 90.

\section{Transistors}

A transistor is a 3-terminal device which has the ability to amplify or regulate electronic signals. The first transistor ever made was a bipolar transistor. It was called bipolar because it employed two kinds of charge carriers: electrons and holes. A hole is a place in the crystal where an electron is missing. A hole behaves as a positive charge. If a semiconductor such as silicon is doped with impurities possessing more electrons than silicon (phosphorus, for example), it is said to be of $n$-type. If it is doped 


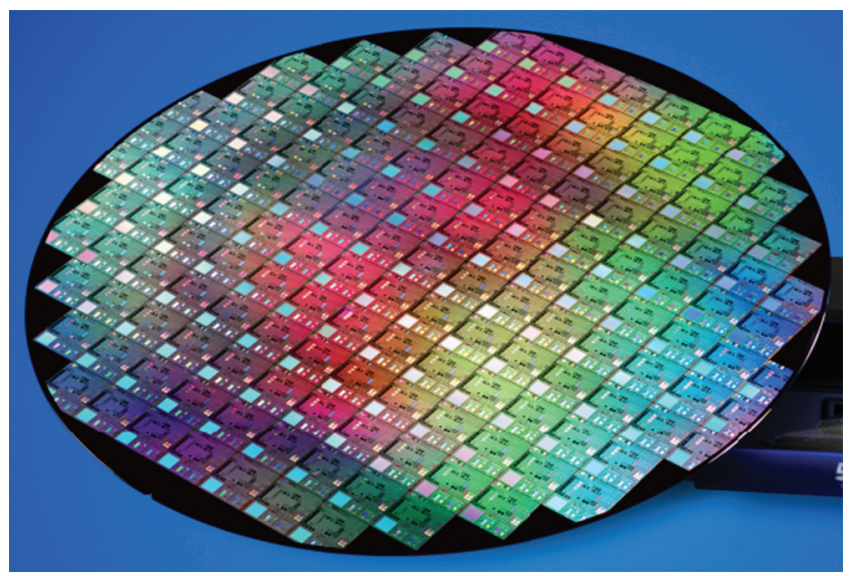

Figure 90 . A $300 \mathrm{~mm}$ silicon wafer with integrated circuits manufactured onto it of is shown. Image courtesy of CEA/LETI, France.

with impurities possessing less electrons than silicon (such as boron), it is said of $p$-type. In a $n$-type semiconductor, electric conduction is done mainly by electron while it is done mainly by holes in the case of a $p$-type semiconductor.

There are several families of transistors. Most transistors manufactured in microelectronics are field-effect transistors (FET) and more precisely MOS-FET (Metal Oxide Semiconductor Field Effect Transistor). The CMOS (Complementary Metal Oxide Semiconductor) technology is widely used in microelectronics. A schematic diagram of an FET transistor is shown in figure 91.

About $10^{20}$ transistors are manufactured every year (about 15 billion transistors per inhabitant). This is larger than the number of grains of rice harvested on the earth each year (There are about 18,00o rice grains per lb. and the global production in 2013-2014 was around 480 million tons).

\section{Moore's law}

Gordon Moore, a co-founder of the Intel Company, noticed, in 1965, that the number of components in integrated circuits had doubled every year since 1958. He anticipated this trend to continue over the next decade. As it turned out, this trend, known as Moore's law, has worked until the present time. Figure 92 shows the evolution of the number of transistors in an Intel processor as a function of the year of introduction on the market. The ordinate being a logarithmic scale, the straight line fitting the data represents 


\section{Principle of a field-effect transistor}

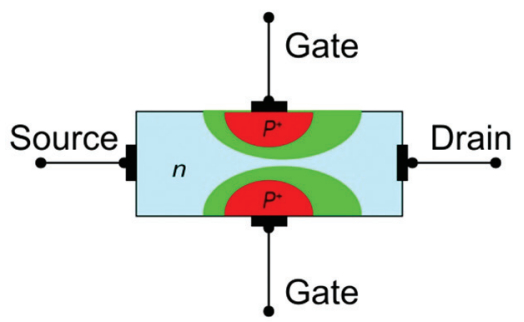

n-channel field-effect transistor

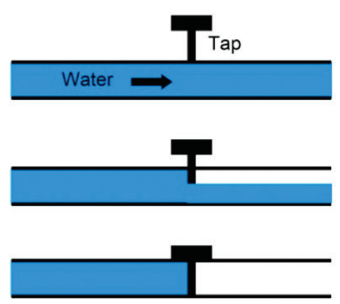

Hydraulic analogy

Figure 91. Left, the principle of an n-channel junction field effect transistor (FET). The red areas are the highly $\mathrm{p}$-doped regions, noted as $\mathrm{p}+$. The light blue region is $\mathrm{n}$-doped. The green region is the depletion region. An increasing value of the gate-to-source voltage increases the depletion region and the channel through which charge carriers flow from the source to the drain is reduced in size. In the right hand side of the figure, a hydraulic analogy to the FET is shown. The tap plays the role of the gate. Depending on the "bias" applied on the gate the flow can be reduced or suppressed.

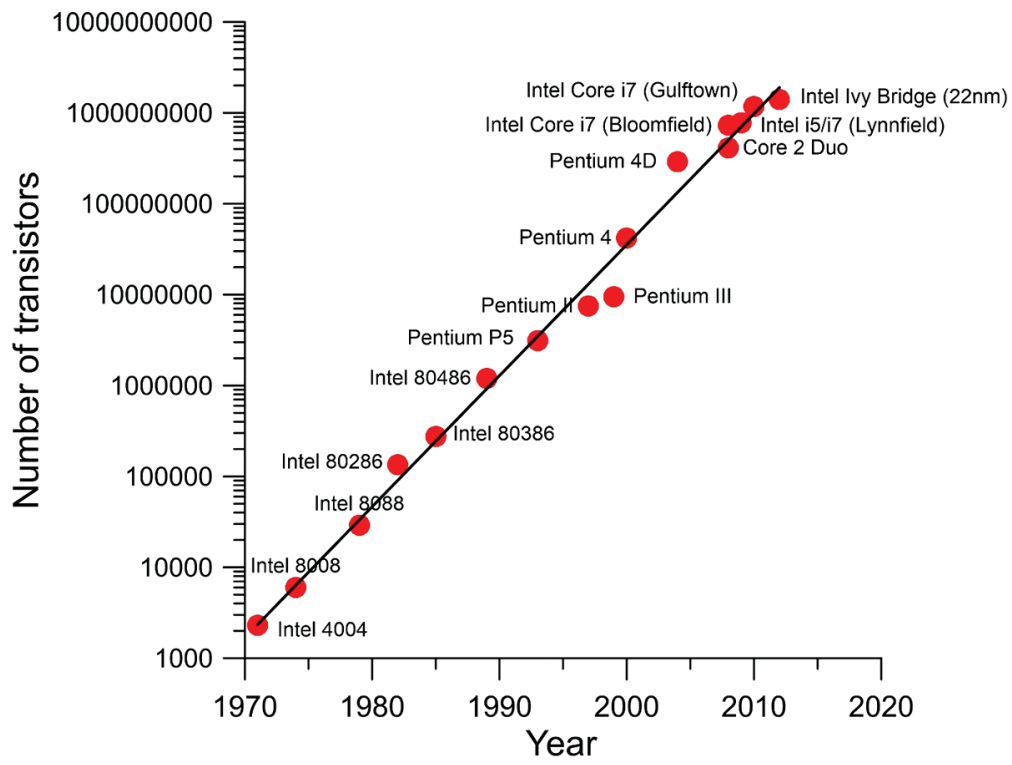

Figure 92. Number of transistors in different Intel processors as a function of their date of introduction on the market. The line shows Moore's law. Since the ordinate is logarithmic, the line corresponds to an exponential growth.

an exponential increase. Overall this agrees with Moore's law. This exponential increase in the processing power per unit of surface on a chip has resulted in an exponential decrease of the cost of a transistor unit. While a 
million transistors on a chip cost about the price of a house in 1973, it cost less than the price of a sheet of post-it notes in 2005 and is even less today. As the cost of transistors has become negligibly small, they have been introduced into more and more objects. Life would be different today without microelectronics.

However, in Figure 92, the change of slope in recent years suggests that the era of Moore's law has come to an end. Further, manufacturing integrated circuits requires higher and higher investments and the cost of a manufacturing plant doubles every 3 years or so. Today the cost of a fab (semiconductor fabrication plant) is commonly around 3-4 billion US dollars. TSMC, a large Taiwan semiconductor company, has recently invested close to $\$ 10$ billion in a wafer manufacturing facility in Taiwan.

\section{Technology nodes}

It has been possible to make smaller and smaller transistors because of the continuous progress in patterning wafers. This has happened in steps called generations, or technology nodes. A technology node refers to the size of the transistors on a chip or half the distance (half-pitch) between two identical features in the case of memories. In 1971, the technology node was at $10 \mu \mathrm{m}$ and in 2011 the technology node was at $22 \mathrm{~nm}$. Figure 93 shows the evolution of the technology nodes on a log-scale. It is now possible to buy commercial microprocessors manufactured with the $14 \mathrm{~nm}$ technology.

We see that the $100 \mathrm{~nm}$ technology node was reached around the 2000's. In a broad sense, it is at this period that one can consider that microelectronics moved into the nanoscale domain.

\section{Memories}

Storage of information is as important as the treatment of information. Most of the memory devices used today to store information are based on magnetic materials. They allow recording of binary information by locally orienting the magnetization of the material in one of two opposite directions. More generally, any material which can be put in two different states can be used to store information.

It is important that memory devices have a high storage density for information, allow the writing and reading of information as quickly as possible, have a low power consumption and have an affordable cost. The 


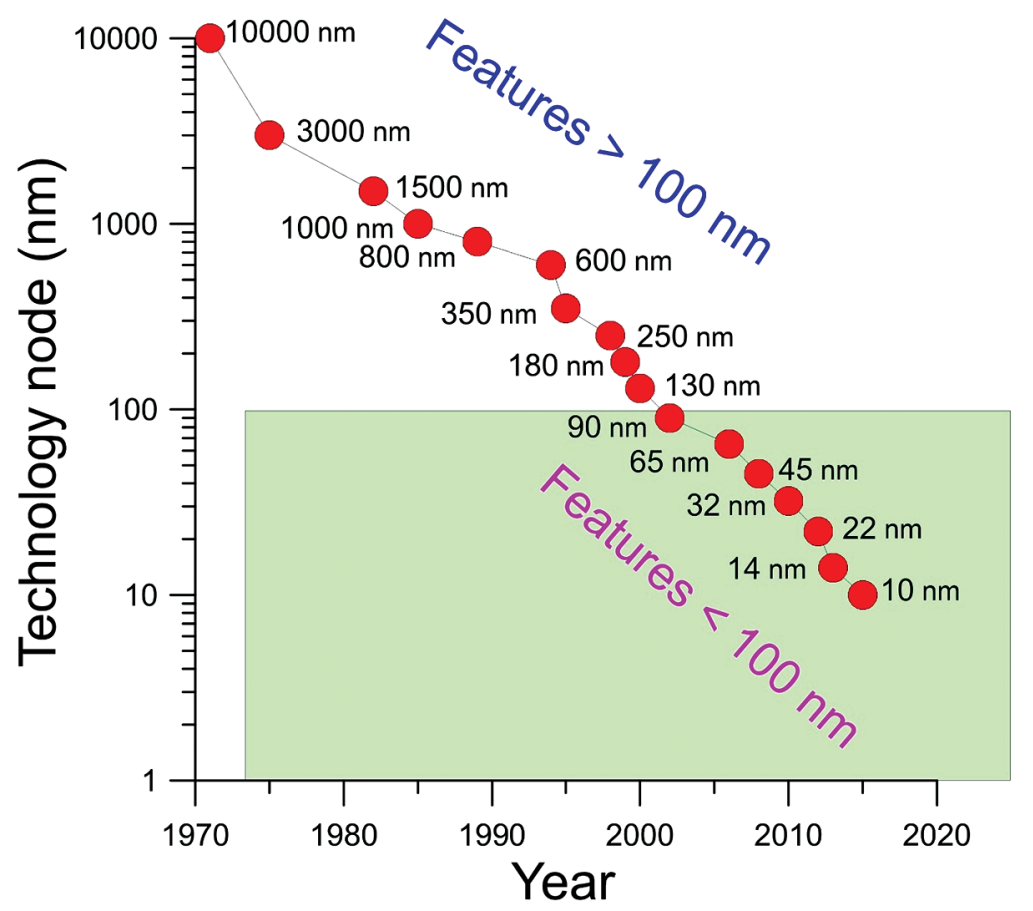

Figure 93. Evolution of the technology nodes in semiconductor manufacturing processes. The ordinate in log-scale.

technical evolution of memories has been similar to that of microprocessors with an increase of about $45 \%$ per year in information density per unit surface.

Three main types of memories are used in computers (figure 94). ROMs (Read Only Memory) are non-volatile memories keeping information when the power is off. They can be written to, but very slowly. In the RAM (Random Access Memory) family there are both DRAMs (Dynamic Random Access Memory) and SRAMs (Static Random Access Memory). DRAMs are extensively used in computers because they are cheap but slow. They require power to keep information and need to be refreshed regularly, SRAMs are fast memories compared to DRAM (about an order of magnitude faster) but expensive. They are called « static » because they keep information as long as the power is on and they do not need refreshing. They require more power in read and write operations.

HDDs (Hard Disk Drives) are well known and used extensively for large storage needs. A hard disk contains rotating disks with magnetic surfaces. Just as for microprocessors, hard disk capacity has increased exponentially 


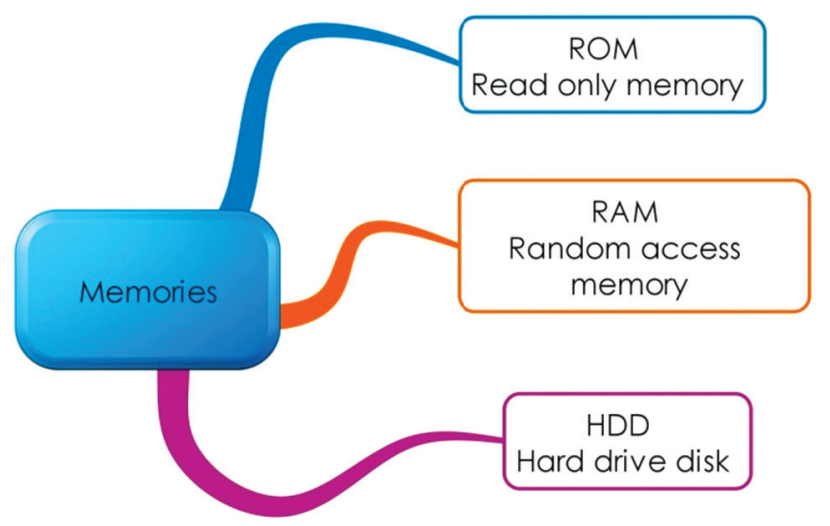

Figure 94. Families of memories used in computers.

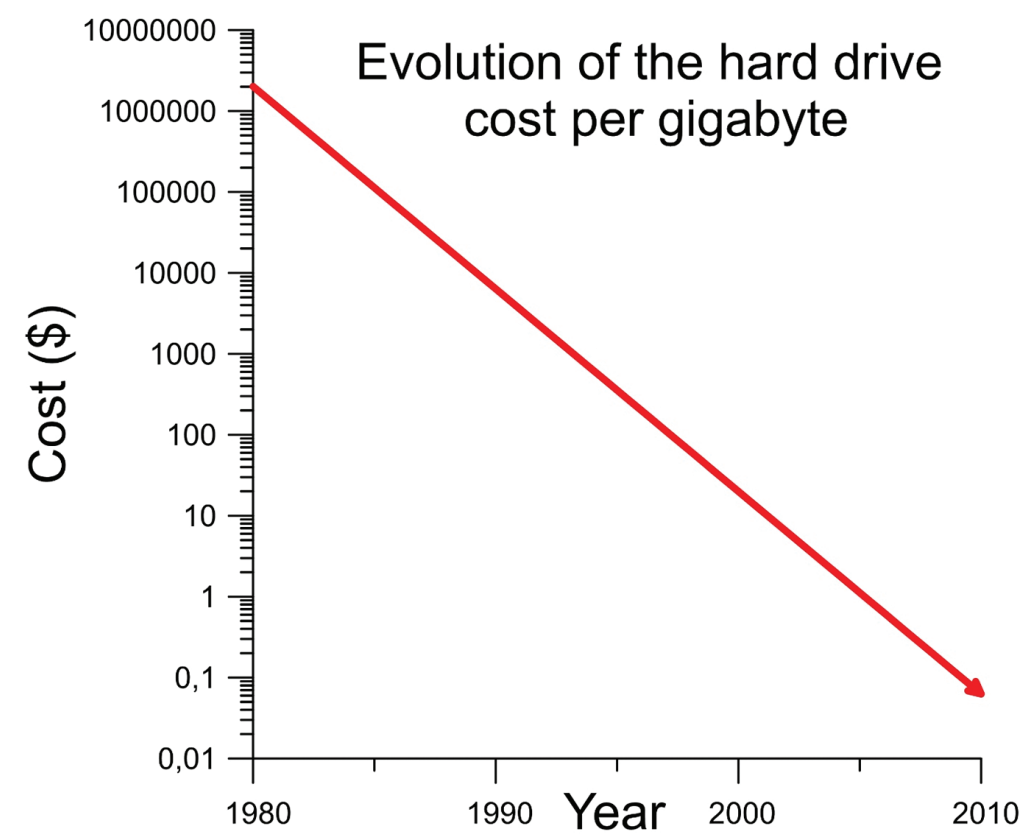

Figure 95. Evolution, on a log-scale, of the average hard drive cost per gigabyte. The curve shown is a best fit of the data performed by Matt Komorowski (www.mkomo.com).

and the cost has dropped exponentially. The evolution of the average cost of a gigabyte of hard disk storage is illustrated in figure 95 .

Decreasing the size of the magnetized domain representing a bit of information increases the storage density. For large domains the magnetization 


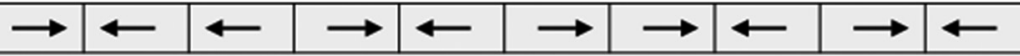

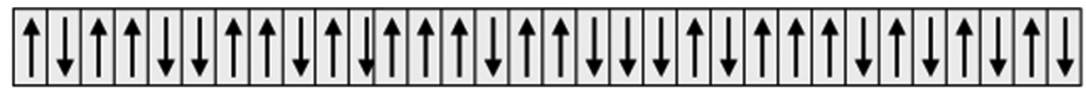

Figure 96. schematic difference between transverse magnetization (top) and perpendicular magnetization (bottom).

is transverse as schematically shown in figure 96. Decreasing the size of the domain leads to a perpendicular magnetization, also shown in figure 96. Perpendicular magnetic recording was introduced around 2005 and has increased the storage density by a factor of 3 . With this technology the storage reliability is also improved.

Today magnetic storage is done using manufactured arrays of nanosized dots. Magnetoresistive materials are used to read and write data on magnetic materials. Spintronics, or spin electronics, is also an emerging area for data storage requiring less energy than conventional magnetic storage. It exploits the spin properties of the electron of which the projection can be either up or down. We shall come back to this subject in the next chapter.

Besides conventional storage technologies where nano-size domains are used, new memory technologies, based on nanotechnology, are also emerging. Most of them are still at the laboratory stage. Some of the most promising are:

- MRAMs (Magnetic Random Access Memories) are non-volatile memories where each cell consists of a fixed magnetic layer separated by a thin dielectric tunnel barrier from a free magnetic layer. Its operation relies on the fact that the resistance of an electric current is lower if the magnetic orientation of the layers is parallel, compared to a situation where they are antiparallel.

- PMCs (Programmable Metallization Cells) consist of a thin film electrolyte sandwiched between layers of inert metal.

- RRAMs (Resistive Random Access Memories) are based on the change of resistivity of certain dielectrics and very fast.

- PRAMs (Phase-change RAMs) use the change of resistivity of a chalcogenide glass as it changes from a crystalline to an amorphous state.

- In Racetrack memories, developed by IBM, information is stored in magnetic domain walls. 
- NRAMs (Nanotube RAMs) use suspended nanotube junctions as memory bits. Applying a voltage moves the nanotube towards the electrode corresponding to a bit state.

\section{Smaller, faster and cheaper}

The driving force of microelectronics can be summarized in three words: smaller, faster and cheaper. This has been true for half a century and will probably continue. The evolution may slow down because the physics changes and new quantum effects which appear at very small dimensions making the components to behave differently compared to what is expected.

Modern microelectronics allows people to employ electronics devices containing on-board intelligence to perform a wide variety of tasks. Smartphones and tablets provide the ability to be connected to the rest of the world at any time and any place, to reach other people, find the latest news, listen to music, watch movies and so on. For these purposes, people seek systems which are increasingly easy to use and have rapid rates of data transfer. This requires ever more powerful electronic devices able to treat more complex information and to store ever more information. Several hundreds of gigabytes of storage will soon be required as an average per person. The requirements for individuals to handle large amounts of information are of course mirrored by those of commercial enterprises. For example, the amount of data generated by Twitter is $7 \mathrm{~Tb} /$ day $(7$ trillion bytes) and that of Facebook of $10 \mathrm{~Tb} /$ day. There is a rapid continuing evolution towards meeting these requirements, thanks to the ability of scientists to produce nanosized components.

Integrated circuits are made on a silicon wafer using lithographic techniques that have continuously improved. The size of the silicon wafers used has increased regularly from 1 " $(25 \mathrm{~mm})$ at the beginning of the microelectronics story, to 12 " (actually 11.8 ") or $300 \mathrm{~mm}$ introduced around 2001 and currently used today. The next generation under development is 18 " or $450 \mathrm{~mm}$ wafers. The thickness of a wafer increases with the size but not as much. For example, it is $775 \mu \mathrm{m}$ for $300 \mathrm{~mm}$ wafers and was $725 \mu \mathrm{m}$ for 200 mm wafers.

As time goes on, semiconductor manufacturers are able to design smaller transistors on larger wafer. If we can make an analogy, it is equivalent to being able to write on a sheet of paper which increases in size with characters that are ever smaller. This is illustrated in figure 97. 


\begin{tabular}{|l|l|}
\hline A & $\begin{array}{l}\text { A wafer is a } \\
\text { thin slice of } \\
\text { semiconduct } \\
\text { or material. A } \\
\text { wafer is a thin } \\
\text { slice of } \\
\text { semiconduct } \\
\text { or material. A }\end{array}$ \\
\hline
\end{tabular}

A wafer is a thin slice of semiconductor material. A wafer is a thin slice of semiconductor material. A wafer is a thin slice of semiconductor material. A

wafer is a thin slice of semiconductor material. A wafer is a thin slice of semiconductor material. A wafer is a thin slice of semiconductor material. A wafer is a thin slice of semiconductor material. A wafer is a thin slice of semiconductor material $A$ water is a thin slce of semiconductor materal. A wafer is a thin slice of semiconductor material. A wafer is a thin slice of semiconductor material. A wafer is a thin slice of semiconductor material. A wafer is a thin slice of semiconductor material. A wafer is a thin slice of semiconductor material. A wafer is a thin slice of semiconductor material. $A$

water is a thin slice of semiconductor material. A

wafer is a thin slice of semiconductor material. A wafer is a thin slice of semiconductor material. A wafer is a thin slice of semiconductor material. A wafer is a thin slice of semiconductor material. A wafer is a thin slice of semiconductor material. A wafer is a thin slice of semiconductor material. A wafer is a thin slice of semiconductor material. A wafer is a thin slice of semiconductor material. A wafer is a thin slice of semiconductor material. A wafer is a thin slice of semiconductor material. A wafer is a thin slice of semiconductor material. A

wafer is a thin slice of semiconductor material. A wafer is a thin slice of semiconductor material. A wafer is a thin slice of semiconductor material. A

Figure 97. Printing analogy to illustrate the evolution of the size of wafers and the decrease of features drawn on them with improving lithography techniques. In the analogy shown here a larger and larger sheet of paper is used to write with smaller and smaller characters.

Portable energy sources, which today rely essentially on batteries that are relatively heavy and expensive remain the weak point in the microelectronics industry. The smaller the transistor, the lower is its energy consumption but when a larger amount of information has to be treated more transistors are usually needed and must be powered.

\section{Summary}

Microlelectronics has become extremely important in daily life. The exponential increase in power of the microprocessors and in the storage capability of memories coupled with an exponential decrease in cost has resulted in the deployment of electronics devices in a large number of areas. Microcomputers can be found everywhere and the internet is pervasive in modern life. This has been possible thanks to a progressive evolution toward nanoscale elements. Details etched with dimensions smaller than $20 \mathrm{~nm}$ are currently available in several commercial products. However, the dimension of the elementary components cannot be reduced forever because new phenomena emerge that will change completely the operation of these small structures. 


\section{Quantum nanoelectronics}

The microprocessors that can be found on the market today are manufactured by etching silicon wafers with a precision as small as $14 \mathrm{~nm}$. Although this precision has dramatically improved over the years today's microprocessors are built with basically the same type of architecture developed for previous microprocessor generations. Reducing the size of elementary components even more could change the nature of the objects involved and they might no longer provide the same functionalities for which they were initially designed. In this size domain, classical physics evolves to quantum physics in many situations and, within this new physical environment, new devices have to be imagined. In this chapter we shall address some of the new phenomena and devices relying on quantum phenomena.

\section{Towards few-electron electronics}

A transistor works as a switch based on electron transport. Electron movement generates signals which are used to carry, store and manipulate information. For example, a bit can be switched from one to zero or vice versa. Several transistors properly associated together can be used to construct logical gates or memory elements. A large number of associated transistors are used to manufacture microprocessors. Shrinking transistors or memories, as it is done in the successive generations of microelectronics technology, reduces energy consumption because fewer electrons are needed to generate digital signals. This is the reason why microprocessors manufactured with smaller components are commonly used in portable computers while those with a less accurate engraving are found in desktop computers.

Electron transport requires energy. The energy is usually provided by the electrical grid or by batteries. In solid state devices such as metal oxide semiconductor field-effect transistors (MOSFET), the number of electrons required to switch or amplify a signal is of the order of 1 to 10 thousand. The efficiency of a single transistor increases as its size decreases. Reducing the distance between the source and the drain (channel) increases the operating speed. Decreasing the thickness of the oxide gate also increases the efficiency. However, below $\sim .2 \mathrm{~nm}$, tunneling effects can occur producing a leak of electrons from the gate electrode to the body and increasing the energy consumption of the MOSFET. To mitigate that effect, the silicon 
dioxide insulating material can be replaced by a material with a higher dielectric constant.

The ultimate goal would be to work with a single electron device. However, as the number of electrons and the size of the device decrease, a change in properties takes place because phenomena that were negligible at large size become dominant at tiny dimensions. Furthermore, using fewer electrons means also that the device is more sensitive to random noise from thermal background, an issue often demanding new operating conditions.

\section{Coulomb blockade}

With the possibility of working at the nanoscale, scientists and engineers have developed one-electron devices which can precisely control the flow of each electron. A fundamental principle underlying one-electron devices was discovered in 1985 by D.V. Averin and A.A. Likharev, two Russian physicists. It is the phenomenon of Coulomb blockade which means that tunneling of an electron through a metallic junction with small capacitance requires an electrostatic charging energy and thus may be inhibited at low temperatures and small applied voltages. The electrostatic potential barrier is a classical concept. Tunneling through the barrier is a quantum effect.

To elucidate this further, let's consider two metal plates separated by a thin layer of insulator such as is schematically shown in figure 98 .

1. Each metal plate is electrically neutral and has an equal number of positive charges (ions) and negative charges (electrons). The net charge of any objectisaninteger multiple of the elementary charge $e$.In other words, charge is quantized. The charge of an electron is minus the elementary charge $-e$ $\left(-1.60217662 \times 10^{-19}\right.$ coulomb $)$ and the charge of a proton is $+e(+1.60217662 \times$ $10^{-19}$ coulomb). The transfer of electrons between two objects always involves a change which is a multiple of the elementary charge $-e$.

2. The system displayed in figure 98 is a capacitor. If a positive charge is applied to one of the metal plates, an equal negative charge appears on the second metal plate. Furthermore, in a metal, the motion of electrons and the vibrations of the ions induces on the metal plates a charge which fluctuates about the mean value as a function of time allowing non-integral values. Thus although the mean charge, $Q$, on a plate is an integer multiple of the elementary charge, when $\mathrm{Q}$ is large it can be treated as a continuous variable. 


\section{Metal Insulator Metal}

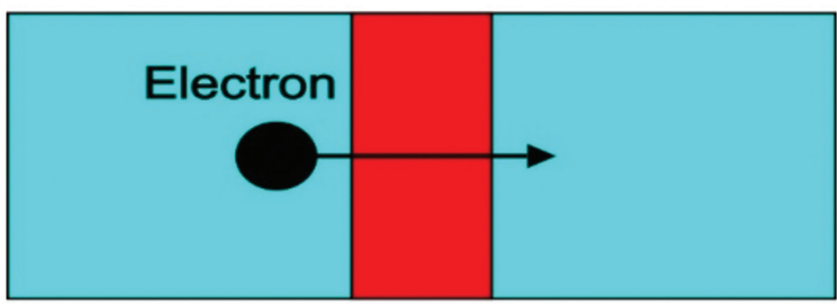

\section{Charge 0}

Metal Insulator Metal

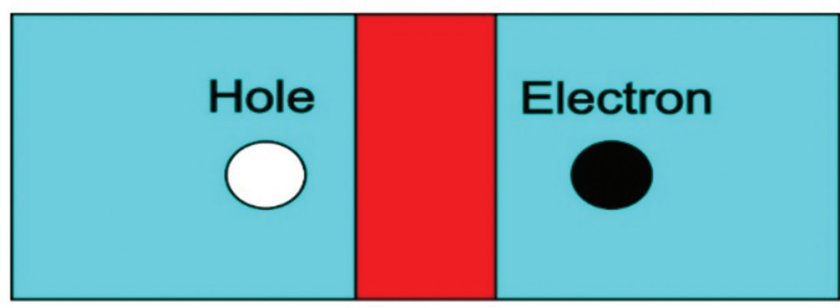

\section{$+e$ Charge $-e$}

Figure 98. Tunnel junction when the two metal electrodes are not charged. An electron cannot spontaneously go across the insulator because it costs energy. Inspired from R.Turton, The quantum dot, W.H.Freeman and Company Limited, 1995.

Consider again the arrangement of two conductors with a very thin insulating layer as displayed in figure 98, but now assume that the device is maintained at very low temperature, typically $1 \mathrm{~K}$ (one absolute degree). The low temperature ensures that the system is always in the lowest energy state and not in an excited configuration due to thermal excitation. If the insulator is thick enough, no current can pass from one conductor to the other. Such a system behaves as a capacitor. As the thickness of the insulator decreases, electron tunneling becomes possible. If we then apply a bias voltage between the two conducting electrodes an electron current can flow. Since the insulator is between two conducting electrodes, the device has both a resistance and a capacitance.

In the top of figure 98, it is assumed that there is no net charge on the electrodes. If an electron tunnels from the left side to the right side there will be one hole of charge $+e$ on the left (an electron missing) and 
one additional electron of charge $-e$ on the right. This is the configuration shown in the bottom of figure 98. Between the initial and final state there is a charge difference of $2 e$. Movement of an electron across the junction increases the energy of the system. The voltage difference $U$ induced by the tunneling is equal to $U=e / C$, where $C$ is the capacitance of the junction. If the capacitance is very small, which is the case for these nanosized systems, $U$ can be large enough to prevent further electron tunneling. A similar situation exists if tunneling occurs from the right to the left. Consequently, if no external energy is provided to the system, no additional electrons can tunnel through the junction. This is the basic idea underlying Coulomb blockade.

Due to the charge fluctuations, it is possible to momentarily have $-e / 2$ and $+e / 2$ on the left and right electrodes, respectively. In such a case, as shown in the bottom of Figure 99, an electron can move from right to left because the charge on the electrodes will just change sign but the energy of the system will remain the same. Interestingly, one electron can tunnel but not two at the same time otherwise an extra energy would be required.

Armed with this insight, we consider the more sophisticated device shown in figure 100. A small metallic dot with a size typically of the order of $100 \mathrm{~nm}$ or below, playing the role of a central electrode, is separated from external electrodes by insulator junctions. If there is no charge in the dot, the electrons cannot go across the junctions because of the Coulomb blockade mechanism. A gate, represented as a rectangle, is used to apply an external voltage. Energy can be injected into the device and the dot can be externally controlled. Applying an external voltage changes the charge on the dot. If this voltage has a value such that it alters the charge to $+e / 2$, the probability that an electron tunnels to the dot increases dramatically. Only a single electron can tunnel and the charge of the dot becomes -e/2. Now the electron in the dot can tunnel out of the dot to the second electrode. This process is known as correlated tunneling. This device allows electrons to go across the dot one by one. The flow of current is determined by the voltage applied to the gate. This works similarly to the gate of a MOSFET.

Figure 100 (1) shows the potential energy of the system as a function of the charge in each of the electrode of a tunnel junction (figure $100(3)$ ). Figure $100(2)$ shows the current as a function of the voltage applied to the junction. Coulomb blockade occurs between $-e / 2 C$ and $+e / 2 C$. The relationship between the current and the voltage does not follow Ohms law since this law would correspond to a straight line in this drawing. 


\section{Metal Insulator Metal}

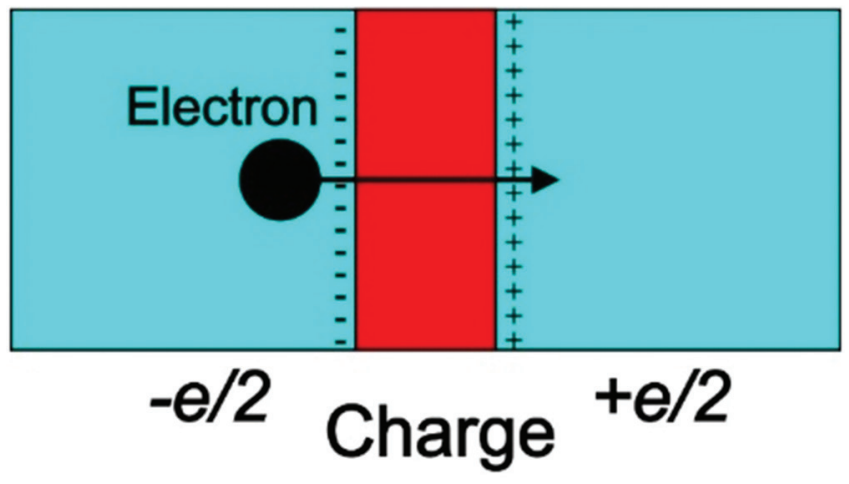

\section{Metal Insulator Metal}

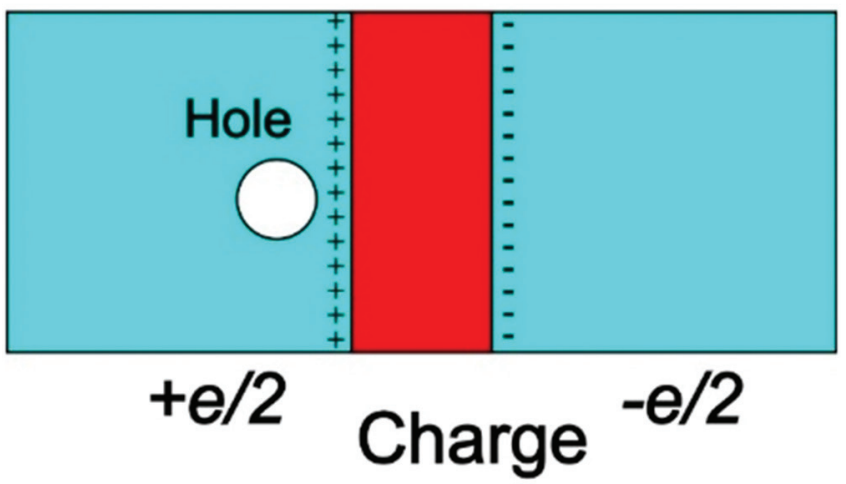

Figure 99. Tunnel junction when the two metal electrodes are charged electrically with a mean charge e/2(reflecting fluctuations). An electron can go across the insulator because it costs no energy. Inspired from R.Turton, The quantum dot, W.H.Freeman and Company Limited, 1995.

\section{The single electron transistor}

In the previous chapter we noted that transistors are three terminal devices used for signal modulation, amplification, voltage stabilization, etc. (See Figure 91). Depending on the type of transistor, an input current or voltage controls the supplied current flowing through the device. We also introduced the MOSFET (Metal Oxide Semiconductor Field Effect Transistor), a widely used unipolar transistor used in digital and analog circuits to amplify and switch signals. The CMOS (Complementary Metal-Oxide Semiconductor) technology is widely used in microelectronics for microprocessors, random access memories, digital logic circuits, etc. The word complementary means 

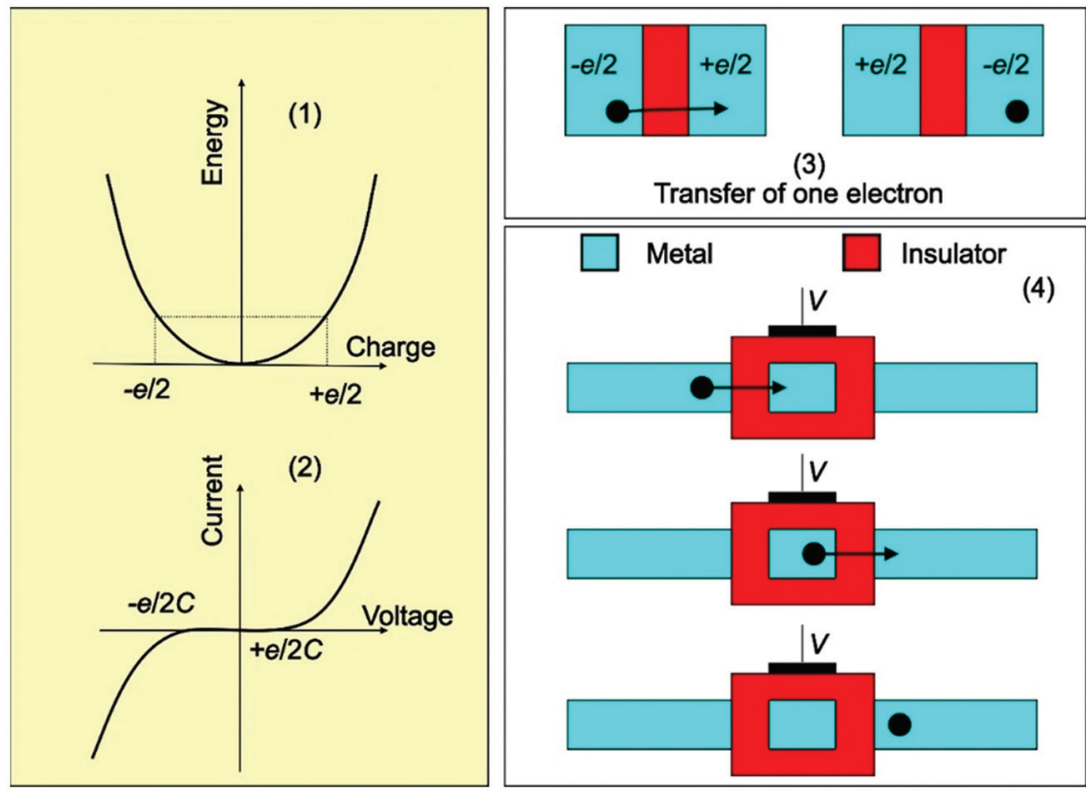

Figure 100. For the system represented in panel (3). Panel (1) shows the energy as a function of the charge of the system. Panel (2) shows the current as a function of the voltage of the system. Panel (4) shows schematically a device allowing the electrons to pass one by one (correlated tunneling). Courtesy of C.Ngô and H.Ngô, Physique des semiconducteurs, Dunod, 4th edition, 2012.

that complementary $p$-type and $n$-type MOSFET's are disposed symmetrically to provide digital functions. The advantages of the CMOS technology are that it is less sensitive to electronic noise than other technologies, and that it has a low power consumption when it is not active.

A single electron transistor (figure 101) is a switching device that has an architecture similar to that of a MOSFET but functions differently. It is a 3 -terminal device consisting of a source, a drain and a very small island of a few nanometers or less built on a semiconductor substrate. The island is electrically isolated from the drain and the source by a thin layer (typically $1 \mathrm{~nm}$ thick) of silicon dioxide playing the role of a tunnel junction. The gate is separated from the island by an insulating layer that is thick enough to prevent tunneling. The gate provides the ability to control the flow of electrons through the island by electrostatic influence. The number of electrons in the island can be precisely fixed and their flow can be completely controlled.

A single electron transistor has to be operated at very low temperature to prevent thermal background that induces thermal excitation because that would make the device useless. The operating temperature increases as 


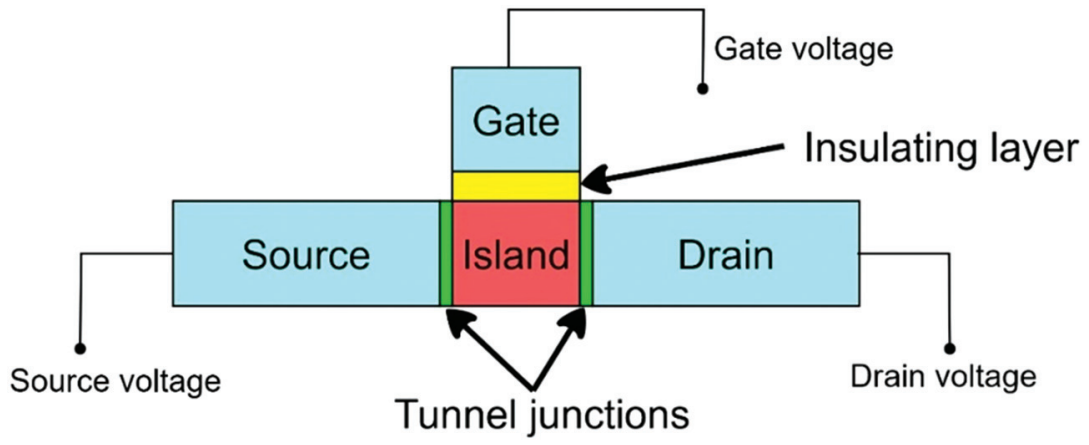

Figure 101. Principle of a single electron transistor. An island, which can be a quantum dot, is separated from the source and the drain by a very thin layer playing the role of a tunnel junction. The gate is separated from the island by a thick layer preventing electrons from tunneling through. The gate electrostatically influences the island.

the size of the island decreases because the available energy levels become more separated from each other (a quantum effect). Working at typical ambient room temperatures requires an island with a size typically of the order of one nanometer or less. The single electron transistor is an emerging technology being developed and studied in laboratories but is not yet at an industrial stage.

The function performed by a single electron transistor is different from that of a MOSFET. Consequently, different architectures must be built to treat the signal information. A wide range of research on single electron transistors built with metal, semiconductors, carbon nanotubes and molecules is underway. An example of a single electron field effect transistor manufactured by the CEA/LETI is shown in figure 102.

Applications for which single electron transistors can be used are: programmable single electron logic, single-electron spectroscopy, DCcurrent standards, electrometers, temperature standards, infrared radiation detection, etc. As an example of achievable sensitivities, a single electron transistor electrometer can detect extremely small DC currents (about $\left.10^{-20} \mathrm{~A}\right)$.

\section{Quantum dots}

A quantum dot is a nanocrystal usually made of semiconductor material in which electrons are confined in a nanoscale volume. Quantum dots were discovered by chance in the late seventies by Alexei Ekinov, a Russian physicist. He was studying the use of different kinds of alloys for microelectronics 


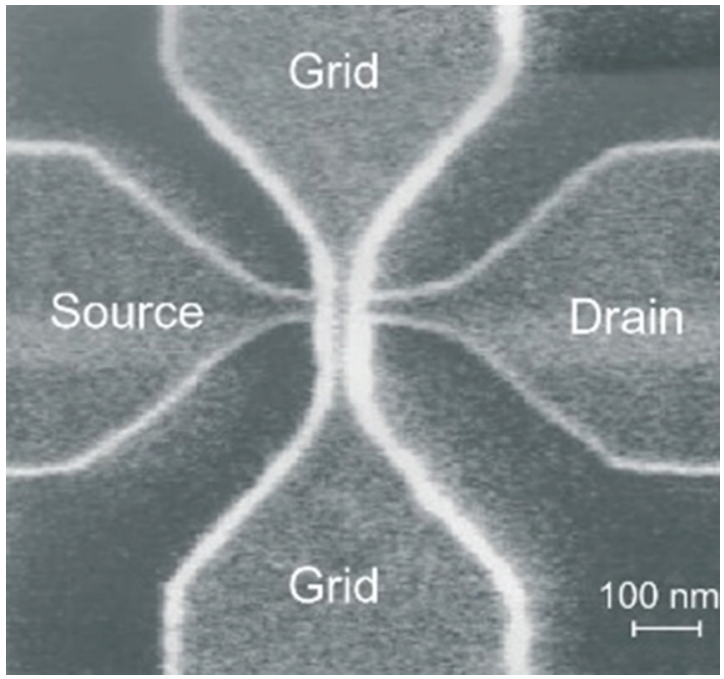

Figure 102. View of a single electron field-effect transistor manufactured in CEA/LETI. Image courtesy of CEA/LETI (France), Clefs CEA n ${ }^{\circ} 52$.

when he noticed some outstanding optical properties of nanoscale crystals of cadmium selenide, CdSe. With this discovery, the standard problem of a free particle enclosed in a nanoscale cubic box, treated in almost all texts in quantum mechanics, became reality.

In a quantum dot, the energy level separation is inversely proportional to the squares of the dimensions of the confining space of the dot. The energy difference between two energy levels is directly connected to the wavelength of electromagnetic radiation which a quantum dot can absorb and the wavelength of emission of electromagnetic radiation from a quantum dot in an excited state. Since it is possible to manufacture quantum dots with a specified size, it is possible to tailor that size to absorb or emit a particular wavelength. For electromagnetic radiation in the visible region, this means that as the size of the dot increases the color of light absorbed or emitted changes.

Figure 103 shows a schematic representation of the color of visible light emitted by excitation of quantum dots of different sizes. The color of emitted light shifts from blue to red as the size of the quantum dot increases. For a spherical quantum dot of diameter $2 \mathrm{~nm}$, light emission is in the blue region while it is the red region if the diameter is about $6 \mathrm{~nm}$.

Figure 104 shows semiconductor nanocrystals of different sizes illuminated with ultraviolet light. The absorbed color is different according to the size of the nanocrystal. One sees the wavelengths and the colors observed during illumination. 

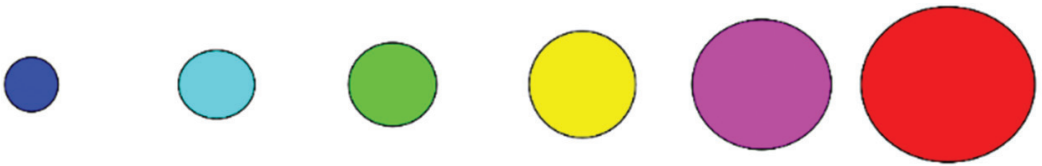

\section{Increasing wavelength}

Figure 103. Schematic illustration of the influence of the size of a quantum dot on light emission. As the size of the quantum dot increases, the color moves from blue to red.

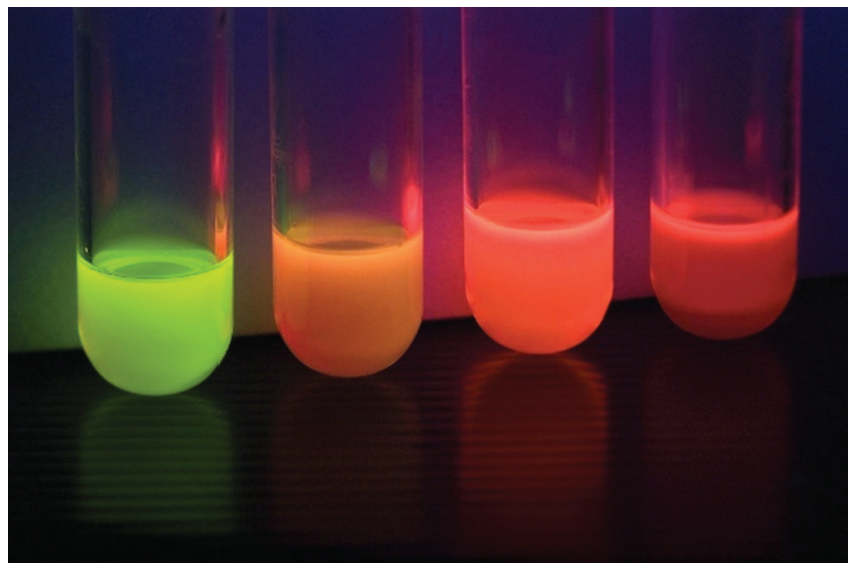

Figure 104. Semiconductor nanocrystals of different sizes illuminated with ultraviolet light. Image courtesy of CEA/LETI (France), Clef CEA n ${ }^{\circ} 52$.

Although quantum dots are usually fabricated from semiconductor materials, it is possible to manufacture a $3 \mathrm{D}$-nanoscale domain out of different materials: metals, insulators, organic substances, etc.

Figure 105 shows, in the left hand part, a quantum dot separated from metallic contacts by a tunnel junction. The top right picture in figure 105 depicts the corresponding energy levels in the potential well. The energy levels in that well can be changed continuously by changing the voltage applied to the gate. An electron located on the left and having an energy that matches that of an energy level of the quantum dot can tunnel through both barriers and go to the right. Because of Coulomb blockage, such electrons will go one by one through the quantum dot and peaks in the conductance will be observed as a function of the gate voltage (bottom right hand side in figure 105).

Because quantum dots are easily tunable they can be adapted to a wide range of applications. 

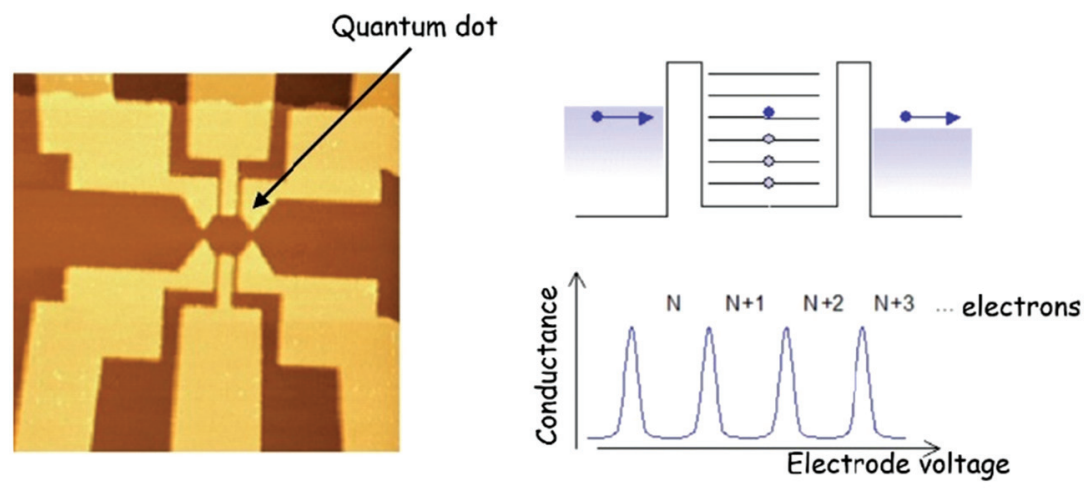

Figure 105. Quantum dot separated from the contacts by a tunnel barrier. The conductance as a function of the gate voltage exhibits peaks corresponding to the tunneling of an electron. Image courtesy of CEA/LETI (France). Clefs CEA n ${ }^{\circ} 5$.

\section{Spintronics}

An electric current in a metal or semiconductor reflects transport of charges that can be electrons (negative charges) or holes (positive charges which are actually arising from missing electrons). The propagation of a hole, an electron vacancy, is schematically illustrated in figure 106 in one dimension.

Spintronics (spin transport electronics) or spin electronics uses the intrinsic spin angular momentum of the electron together with its charge to develop new kinds of electronic devices. Associated with this spin is a magnetic moment. As a result the spin of the electron can be oriented with a magnetic or electric field.

The spin, $\mathrm{s}$, of the electron is $1 / 2 \hbar$ and two projections on the $z$ axis are possible: $s_{z}=+1 / 2 \hbar$ and $s_{z}=-1 / 2 \hbar$ often referred to as up (spin up) and down (spin down). Exploiting the spin of an electron to carry and store information is interesting because it can be done rapidly with a low energy consumption.

Spins can be spatially arranged differently depending upon the external conditions. If the electrons are free to move at ordinary temperatures, they are randomly distributed as illustrated in the left hand part of figure 107. Applying a strong magnetic field in the proper direction can align the spins as illustrated in the right hand part in figure 107.

The spins in a crystal made of non-magnetic material can be oriented at random. In magnetic materials they are aligned (see figure 108). 


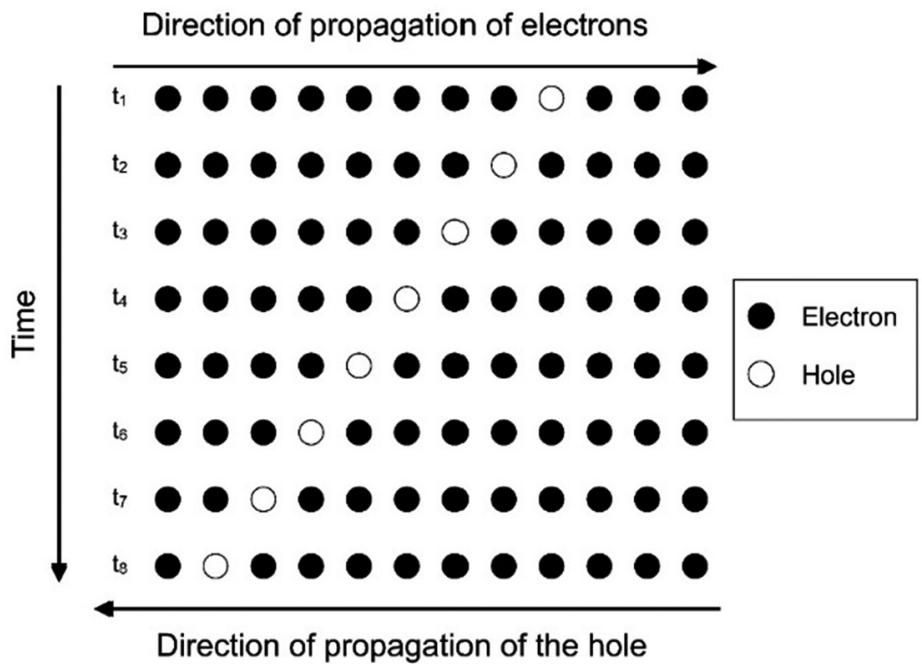

Figure 106. Propagation of a hole (site where an electron is missing) for a 1-dimensional lattice of electrons. Electrons are represented by solid black circles and the hole by a white circle. Snapshots of the lattice are shown at increasing times $\mathrm{t} 1<\mathrm{t} 2<\ldots<\mathrm{t} 8$. As electrons move to the right the hole moves to the left.

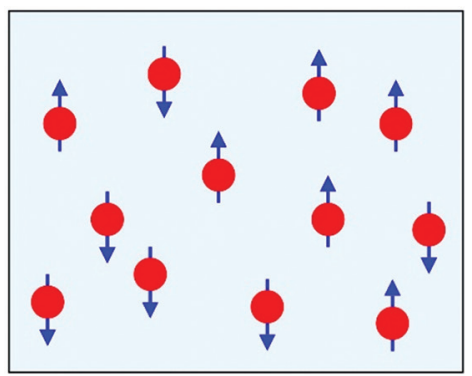

Random spins

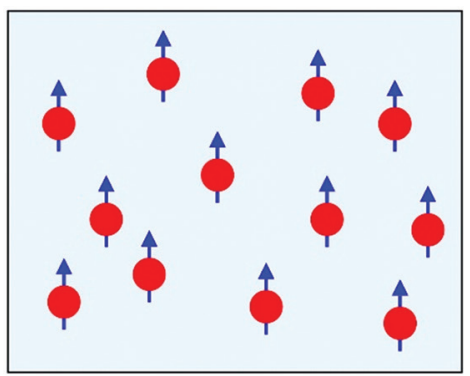

Spins aligned

Figure 107. In the left-hand part the spins are oriented at random along the same direction while, in the right-hand part, they are aligned along a strong external magnetic field. Inspired from S.D.Sarma, Spintronics, American Scientist, Vol 89, 516, 2001.

Magnetoresistance, discovered in 1856 by Lord Kelvin, is a physical effect in which a change in electrical resistance of a material occurs in response to an applied external magnetic field. The possibility to fabricate layered materials in the nanometer range led to the discovery of "giant magnetoresistance" (GMR). It is a quantum effect independently discovered in 1988 by A.Fert and his team in France, and by P.Grünberg and his team in Germany. They were awarded the 2007 Nobel prize for this discovery. 


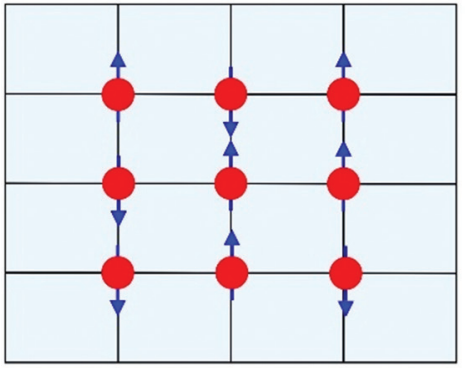

Unmagnetized

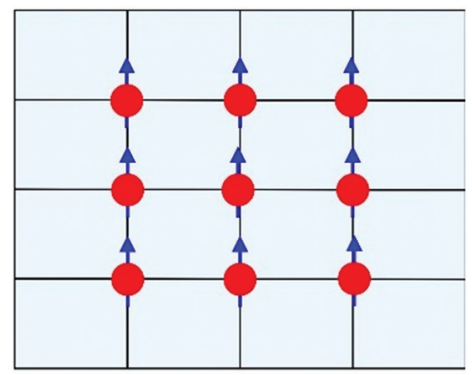

Magnetized

Figure 108. In a crystal atoms are located at specific sites. The spins will be oriented randomly in the case of an unmagnetized material or will be aligned in a magnetized material. Inspired by S.D.Sarma, Spintronics, American Scientist, Vol 89, 516, 2001.

Ferromagnetic material (Co)

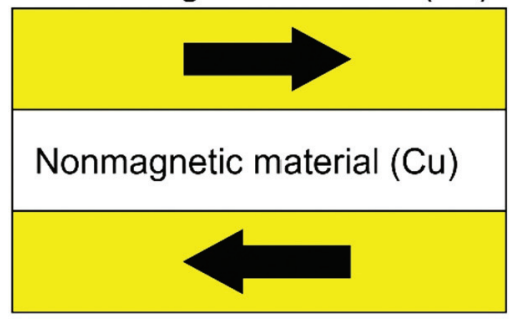

Ferromagnetic material (Co)
Ferromagnetic material (Co)

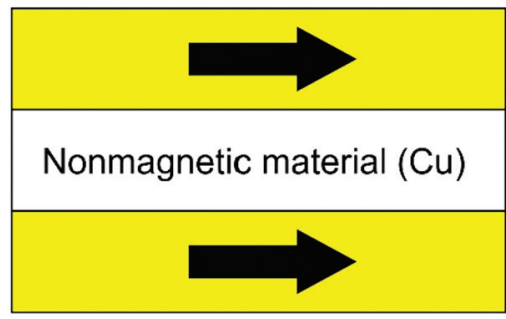

Ferromagnetic material (Co)

\section{High resistance}

\section{Low resistance}

Figure 109. Principle of giant magnetoresistance. The electrical resistance is high when the magnetizations of the ferromagnetic layers is antiparallel, while it is small when the magnetizations of these two layers are parallel.

The effect is illustrated in figure 109 using a stack of only three layers. In real experiments 10 layers or more may be involved. In the figure, a nonmagnetic layer with a thickness of the order of a nanometer is sandwiched between two ferromagnetic layers. The electrical resistance varies strongly with the relative orientation of the magnetization of these ferromagnetic layers. The electrical resistance is very high if the two magnetized layers are antiparallel (or in the case of a greater number of layers, when the ferromagnetic layers have alternating orientations of the magnetization) while is becomes very small for parallel magnetization. 
Basically, electrons with parallel spin have small scattering probabilities giving a small electrical resistance while electrons with antiparallel spins have a high probability of scattering leading to a high electric resistance. The electron mean free path, i.e., the average distance between two collisions, is of the order of 10-100 $\mathrm{nm}$. In order that the GMR mechanism appears, it must be much larger than the interlayer separation which is the case when layers with thicknesses of the order of $1 \mathrm{~nm}$ or so are involved.

Applications of the GMR include memory storage (hard disks) and magnetic field sensors. In the case of hard drives, the spin up and spin down states provide the natural basis for binary coding of information ( 0 and 1 ). Since 2000, spin-valve sensors, exploiting the GMR effect have been used in $100 \%$ of the commercial hardrives.

With spintronics, there are other possibilities than the magnetic field to control magnetization. It is possible to control magnetization with spinpolarized electric currents, electric fields or photonic fields. Spintronics devices are very small and very fast, operating on time scales of picoseconds or femtoseconds.

\section{Nanophotonics}

The US academy of sciences defines nanophotonics as "the science and engineering of light-matter interactions that take place on wavelength and sub-wavelength scales where physical, chemical or structural nature of natural or artificial nanostructured matter controls the interactions". The wavelength of visible light is in the range of about $380-780 \mathrm{~nm}$. This is far above the nanoscale domain which has been defined as the range 1-100 $\mathrm{nm}$. Nanophotonics is concerned with interactions controlled by physical or chemical properties of nanostructures or by their structure.

Nanophotonics can be divided in three main areas depending on the nanoscale confinement involved. The first area deals with the confinement of light. This includes photon localization in photonic crystals, the control of light propagation and light-matter interactions. Near field optical microscopy or photon scanning tunneling microscopy belong to this area. The second area deals with the confinement of matter into $1 \mathrm{D}, 2 \mathrm{D}$ and ${ }_{3} \mathrm{D}$ nanostructures. Nanophotonics provides the ability to control the optical behavior and the functionalities of these devices. The third area is the confinement of nanoprocesses. This deals with the nanofabrication and synthesis of devices such as nanoscale sensors, detectors and actuators or nanoscale lasers, etc. 


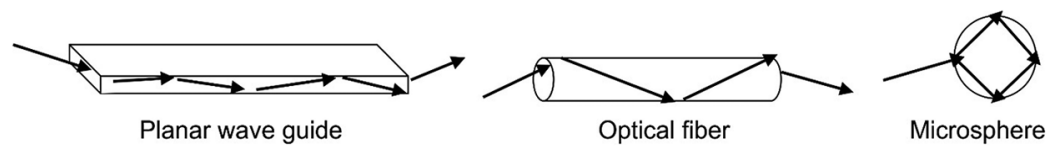

Figure 110. Schematic representation of different light confinement geometries. A planar wave guide corresponds to 1D confinement, an optical fiber to a 2D confinement and a microsphere to a 3D confinement. Inspired by A.Sharma, Nanophotonics, an overview, NSF-RISE workshop (2007).

\section{Confining and Controlling light}

Waveguides such as those illustrated in figure 110 have long been used to confine and direct light. This is with confinements operating in different space dimensions.

A waveguide usually has a structure in which a material with a high index of refraction is surrounded by a material with a low index of refraction that constitutes the cladding. Waveguides can be used in optical connections. Optical connections are interesting because light propagation in a waveguide is faster than electron transport in a conducting wire. Furthermore, the energy dissipation in the light propagation is negligible compared to energy dissipation in the electron transport (the Joule effect). However, it should be kept in mind that if light has to travel over much larger distances than electrons, the global device can be slower. In addition, the coupling between optical interconnections and conventional electrical circuits require conversion of a light signal into an electronic signal and vice versa. This requires new coupling devices that may dissipate energy and introduce time delays.

On the nanoscale, antireflection coatings and Bragg mirrors which consist of alternating layers of two different optical materials, have been used for a long time. In 1978, the first quantum well laser working at room temperature was built. The active area was only $20 \mathrm{~nm}$ thick. Quantum well lasers are now common.

\section{Photonic crystals}

Photonic crystals are artificial periodic nanostructures with spacing of the order of the optical wavelength. Before we go further it is useful to recall a few things about electrons in crystals.

A single atom has discrete levels of energy as shown in the left hand part of figure 111. These levels define the only possible values of energy that 

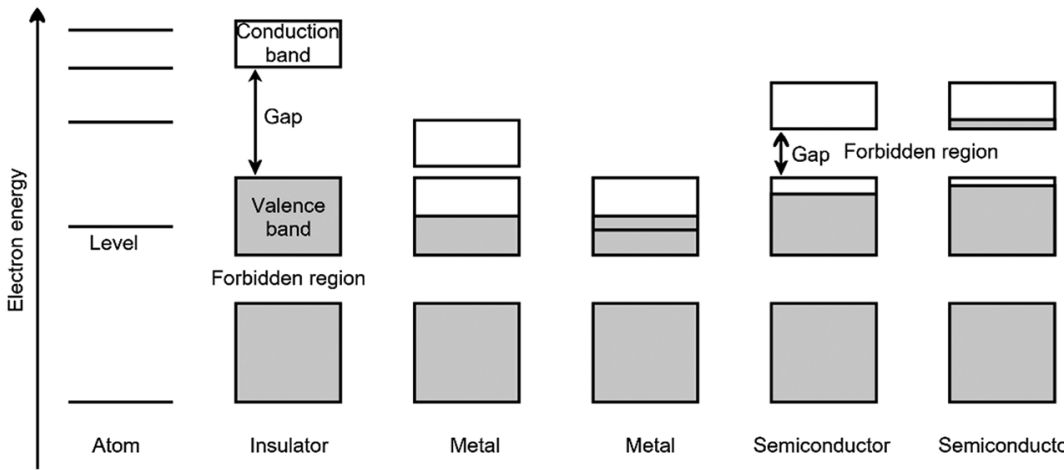

Metal

Metal

Semiconductor

Semiconductor

Figure 111. Schematic representation of the band structure of metals, insulators and semiconductors. In the left-hand side of the figure, the level structure of a single atom is indicated.

an electron in the atom can have. Other energies are forbidden. This is a direct result of the quantum mechanics that we discussed in chapter 2 . In a perfect crystal with atoms located at each site of the lattice, the energy levels transform in bands as shown in figure 111. Electrons of the crystal can only have energies belonging to a band. The regions between bands are forbidden. In the same way as the energy levels of an atom are filled from the bottom when satisfying the Pauli principle, bands are filled with the available electrons starting from the bottom. The highest energy band containing electrons is called the valence band. The lowest unoccupied band is called the conduction band. The energy difference between the top of the valence band and the bottom of the conduction band is called the band gap or energy gap.

In insulators, the valence band is completely filled with electrons and the gap is large, preventing valence electrons from being promoted into the conduction band under normal conditions. In a metal, either the valence band is incompletely filled or there is an overlap between the valence and the conduction band. In the intermediate situation, the valence band full, the conduction band empty and the energy gap small, the material is a semiconductor.

Most modern microelectronic devices use semiconductors, mostly silicon, to carry out switching and logic functions. The availability of electrons and holes in the valence and conduction bands is controlled by using dopant atoms in very small quantities. These dopant atoms possess either more or less valence electrons than silicon.

Photonic crystals, proposed almost simultaneously in 1987 by E.Yablonovitch and J.Sajeev, have some structural analogies with semiconductors or insulators. In a semiconductor, there is a periodic potential that is at the 


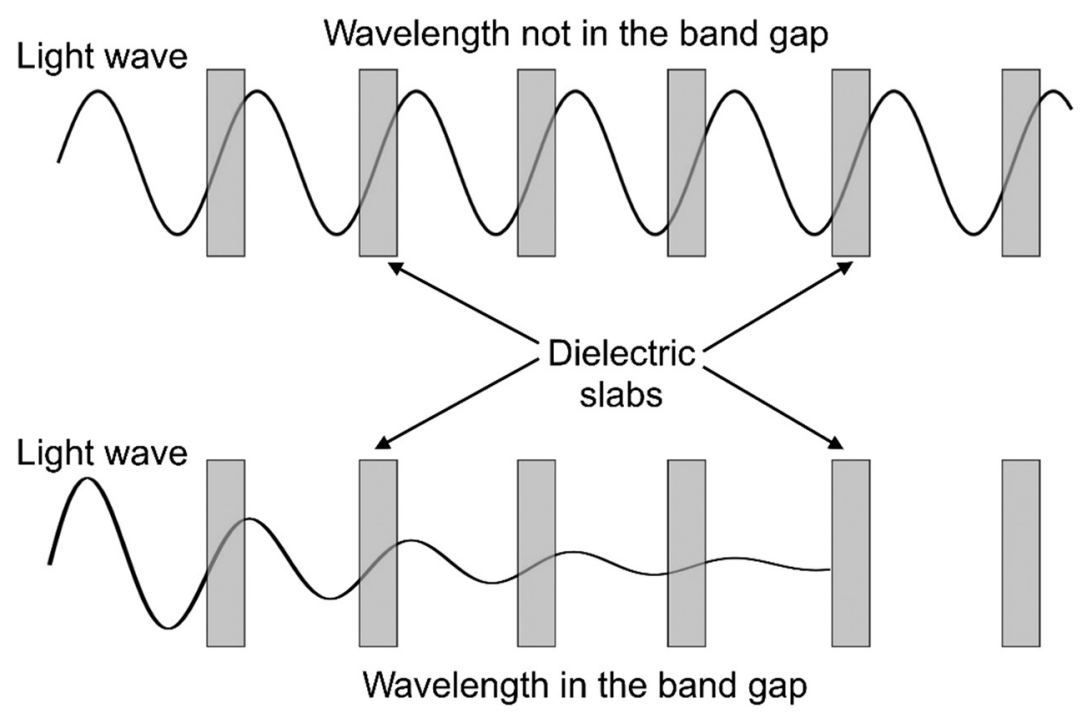

Figure 112. Basic principle of a 1D-photonic band gap material. When the wavelength is not in the band gap the incident light wave can go through the material with little attenuation. When the wavelength is in the band gap of the material, the incident light cannot go through. This comes from the fact that the reflected waves on each dielectric slab are in phase and reinforce each other.

origin of forbidden band gap for electrons. In a photonic crystal there is periodic dielectric function created by pieces of material with a different dielectric constant. This produces a periodic index of refraction that can give rise to a photonic band gap. By properly choosing the dielectric material and arrangement, it is possible to tailor the photonic bandgap. A photonic band gap serves to light in a certain wavelength range of.

Figure 112 shows the principle of a photonic band gap in a $1 \mathrm{D}$ periodic arrangement. In the $1 \mathrm{D}$-crystal, slabs of transparent dielectric material are separated from each other by air, for example. If the wavelength of the incident light is not in the band gap, light propagates through the material with very little attenuation. The incident wave is partly reflected by each slab but these waves are out of phase and cancel one another. If the wavelength is in the band gap, the reflected waves are in phase and reinforce each other. Light is blocked by the crystal.

The nice thing is that photonic crystals can be engineered on demand giving the ability to tailor the properties of the photonic band gap. In creating a photonic crystal, it is possible to play with the dimensionality of the crystal, the symmetry and topology of the lattice, the lattice parameter, the filling fraction (relative amount of refractive material), the refractive index contrast (ratio between the high dielectric constant of the material located 


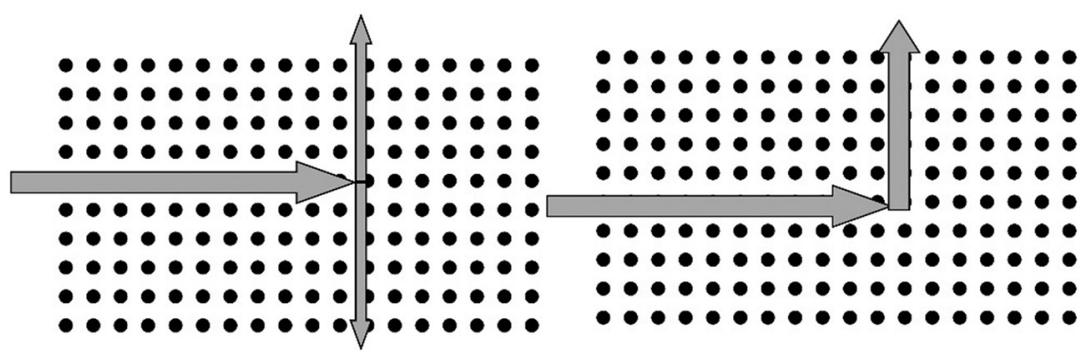

Figure 113. Schematic examples of waveguides using photonic crystals. In the left hand part, a wide-angle splitter is shown and in the right hand part a lossless sharp bend wave guide. The lattice is represented by small black dots which correspond to high dielectric material. The light wave is represented by grey arrows.

at the sites and the low dielectric constant of the remaining material) and the scalability that allows similar properties at different wavelength.

Applications of photonic crystals include supercollimators or superlenses, light confinement of (near-field and far-field microscopies), photonic integrated circuits in which photons, not electrons, carry information etc. Figure 113 shows two examples of wave guides that can be obtained with photonic band gap crystals.

Optical fibers based on photonic crystals are already commercially available. Bragg gratings are simple examples of a $1 \mathrm{D}$ photonic crystal. They have many applications, among them measurements of the structural stability in dams in order to prevent accidents.

\section{Plasmonics}

In metals, light can couple to electrons to create a wave bound to the surface of the metal that is called a surface plasmon. This provides a way to manipulate light at length scales smaller than the wavelength of the incident photons. It occurs at the interface between a dielectric such as silica glass or air, and a metal such as silver or gold. The dielectric has a positive dielectric constant while a metal has a negative dielectric constant.

A surface plasmon is a coherent electron oscillation propagating at the interface between the metal and the surrounding environment together with the electromagnetic wave associated to the incident light. In the same way as photons are the quanta associated to light, plasmons are the quanta associated to plasmon oscillations. Actually, these quanta are quasiparticles in the same way as phonons, the quanta associated with lattice vibration, 
are quasiparticles. A quantum dot, or a light emitting diode, located close to a metal surface can excite surface plasmon oscillations.

The wavelength of surface plasmons turns out to be much smaller than the wavelength of the exciting photons (typically an order of magnitude less). For example, a He-Ne laser with a wavelength of $633 \mathrm{~nm}$ can excite a surface plasmon oscillation at $73 \mathrm{~nm}$ at a silica-silver interface. By tuning the laser wavelength around the plasmon resonance, it is possible to decrease the plasmon wavelength down to the nanometer range. With such a capability it is possible to make plasmonic nanocircuits which treat light on dimensions smaller than its wavelength. Because of this, nanowires can guide light well below the diffraction limit, metal films with nanoscale holes have high optical transmissions and very thin films can be used as optical lenses.

\section{Metamaterials}

Metamaterials are engineered materials that have properties not found in nature. They are usually a complex association of components forming a composite material that can be metal-like, plastic-like or ceramic-like. The new properties are obtained from the geometrical arrangement, size, orientation, etc. of the individual elements rather than by the properties of these individual elements. In particular, these properties allow the metamaterials to block, absorb, enhance or bend electromagnetic or sound waves in a way that is not possible with conventional materials. In the domain of metamaterial, nanotechnology plays a preeminent role in constructing nanostructures that exhibit these smart properties.

In photonic crystals which have a lattice size of the order of the wavelength of light, diffraction limits the resolution. It is possible to make lattices of metamaterials with lattice constants much smaller than the wavelength of light and avoid the diffraction limit. A very active domain of research in the area of metamaterials explores negative-index of refraction materials. With such materials, negative refraction, in which light is refracted at the interface in the opposite sense to that normally expected, can be observed (figure 114).

Applications of metamaterials are possible in several areas: telecommunication, sub-diffraction imaging, sensing or invisibility cloaks. This last domain is very exciting to the general public. To be invisible, it is at least required that the refractive index of the invisible body is equal to the refractive index of air. In some wavelength regions, this can only be achieved using plasmonic metamaterials. 


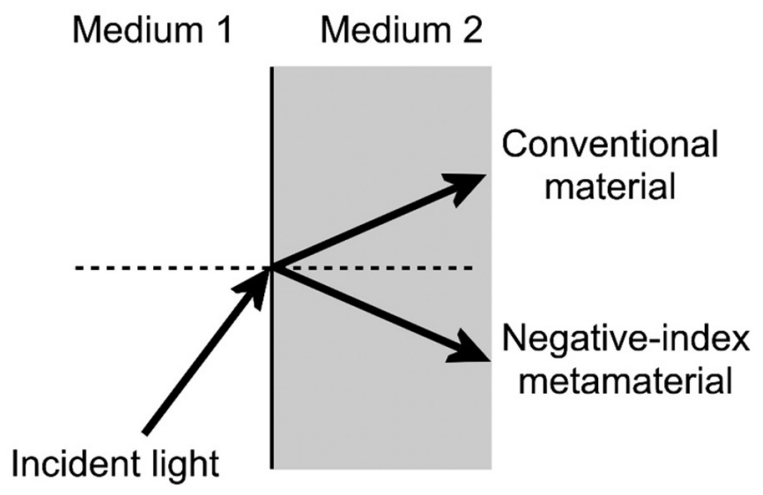

Figure 114. Diagram illustrating the difference of refraction in a medium (medium 2) having a positive-index (conventional material) or a negative-index (some metamaterials).

\section{Summary}

The appearance of new phenomena at the nanoscale paves the way to new applications. Some applications, e. g. devices based on giant magnetoresistance are already commercialized. Others such as the single electron transistor are still in the laboratory stage. Quantum nanoelectronics, which does not correspond to a simple extrapolation of conventional microelectronics, offers new opportunities to treat or store information. When the function that is possible is new or more efficient than a similar one in conventional electronics, it can be incorporated into a microdevice (nano-inside). The integration of some quantum nanoelectronic functions into conventional devices will probably be the future evolution of microelectronics:

In the more distant future, the further evolution of quantum nanoelectronics may make quantum computing available at large scale. Quantum computers are for the moment at the research stage. They use Qbits (quantum bits) which are not limited to two states as it is the case with bits used in conventional computers. Quantum cryptography, which is an application of quantum entanglement, a non-local property of quantum objects, could be used to make unbreakable cryptosystems, also an interesting application. 


\section{Molecular electronics}

Microelectronics and nanoelectronics are made by top-down approaches. Another way to manufacture nanoscale devices is to use atoms, single or group of molecules, carbon nanotubes, metallic or semiconductor nanowires as the elementary components and find methods to assemble them in the right way to perform electronic functions. This is the essence of molecular electronics called also moletronics. Sometimes, the term molecular electronics is restricted to the use of single molecules only but, for applications, it is better to use an extended definition involving nanoscale components. It is not an easy task to develop bottom-up approaches because nanoscale objects already contain a large number of molecules and atoms and the main issue is to assemble them correctly and quickly in the right way.

Organic molecules are often used in molecular electronics because they offer an enormous number of possibilities. One advantage of bottom-up approaches is the ability to choose starting material from large number of organic molecules that are already characterized. Another is that their properties can be tailored using chemical synthesis techniques. Since specific molecules can be precisely produced by chemical synthesis, the bottom-up process can be initiated with identical molecules. However, assembling them to get a nanodevice is not straightforward. Self-assembly is often used because it provides the ability to manufacture complex systems at a high speed.

The fundamental question of molecular electronics science is to understand how electrons move through a molecule. Historically, laboratory studies have long addressed that question. However, the field of molecular electronics really started in 1974, when A.Aviram and B.Ratner first proposed the idea of a molecular rectifier.

\section{Electronic conduction}

From the fundamental point of view, the first aspect to be understood is how a charge moves from one side of a molecule to the other. Two things are needed for electron conduction in inorganic materials. First, it is necessary to have a large number of orbitals interacting strongly with each other so that a band structure can be obtained (see figure 111). This is obtained in lattice structures. Second, there should be enough electrons to fill these bands. These two conditions are fulfilled by metals and semiconductors. 


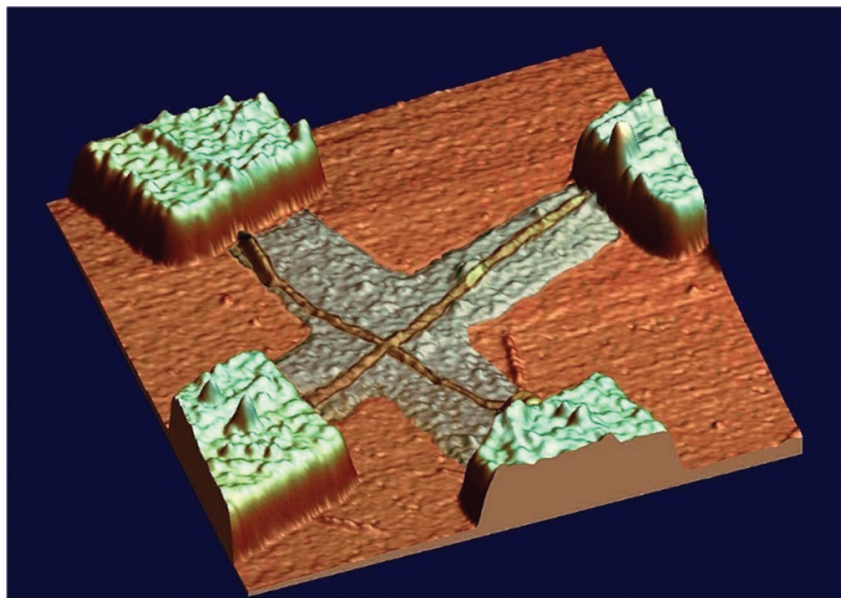

Figure 115. Crossover of two carbon nanotubes deposited on silica. The aim of this experiment, a joint work by CEA and MOTOROLA, was to illustrate the production of nanotube circuits by selfassembly. The gray strips are molecular glue used to position the nanotubes. Image courtesy of CEA/LETI (France), Clef CEA n52.

In the case of organic molecules, delocalized electronic states are necessary. In addition, other conditions may be required. For example, conjugated polymers are conducting only if they are doped.

A second aspect is to study and understand how a current passes through a molecule that is connected at each end to an electrode. An important issue is how to electrically connect a molecule to the external world. It is necessary to do that to measure electrical properties and to build complex electronic devices. Gold or platinum are normally used to manufacture electrodes because they are resistant to oxidation and degradation. Sulfur or amine molecules can be used to attach the molecules to gold. Molecules with unbonded pairs of electrons are often used with both gold and platinum electrodes. Figure 115 shows an example of a laboratory experiment to investigate the crossover of two nanotubes.

Different mechanisms are involved in the electric conduction of molecules. Conductivity can arise from quantum tunneling or by hopping conduction in which a charge carrier has the ability to move between localized states.

\section{Difficulties}

Even if performing a logical function is possible with a molecule, further conditions are necessary for this function to be realized or included 
in a more complex electronic system. Consider, for example, one of the simplest functions that can be imagined: a molecular switch. This could be a molecule that can exist in two different conformational states. One state would correspond to the switch "on", the other to the switch "off". In order to be useful, the molecular switch should fulfill the following conditions:

- The switch should change state on demand and not spontaneously.

- The transition from "on" to "off" has to be reversible otherwise that would be a one shot switch

- The switch has to be addressable, which means that, if there are a large number of switches available, we have to be sure to activate this switch and not another one. Since the switch molecule is generally positioned among other molecules, one has to be sure that it stays in place and does not move to another place due to thermal motion.

- The switch should be inert with respect to chemical reaction with other molecules and remain stable under the ambient external conditions.

All these conditions put heavy constraints on the molecular properties and on the architecture of the device that makes it difficult for this field to reach large scale development.

\section{Molecular wires}

Electronic conduction in molecules is possible if there are overlapping orbitals allowing a delocalization of electrons over the whole molecule. Electrons moving in delocalized electron orbitals do not belong to a single bond or atom, but rather to a group of atoms. Such molecules can be used as molecular wires. The simplest structure that can be designed consists of an organic molecule that is a chain of alternating single (two electrons, sigma) and double (four electrons, pi) bonds (Figure 116, top). Such a molecule is said to be a conjugated system. In this case, the $\pi$ electrons are delocalized over the whole chain. More complicated structures involving aromatic components, i.e., rings of conjugated bonds, can also be made.

In Figure 117 the six carbon atom structures $\mathrm{C}_{6} \mathrm{H}_{6}$, benzene and $\mathrm{C}_{6} \mathrm{H}_{12}$, cyclohexane are depicted. The benzene molecule is a conjugated aromatic molecule. Although a benzene ring cannot be used alone in molecular electronics, one or several benzene rings can be associated to other functional groups to make complex molecules that can be used for 


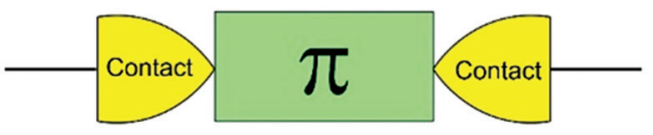

Molecular wire

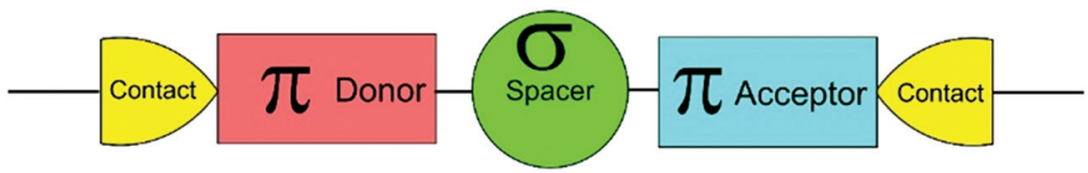

\section{Molecular diode}

Figure 116. Top of the figure: principle of a molecular wire. The rectangle named $\pi$ corresponds to the molecular wire. It is a molecule with delocalized $\pi$-electrons. Bottom of the figure: Basic principle of a molecular diode. The circle named $\sigma$ is a part of the molecule with only $\sigma$ electrons (electrons localized in bonds).

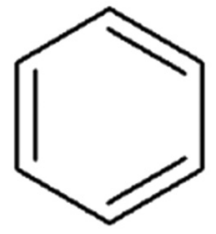

Benzene

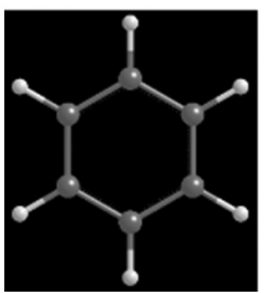

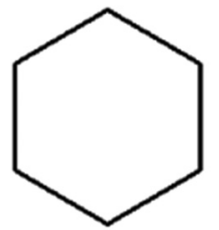

Cyclohexane

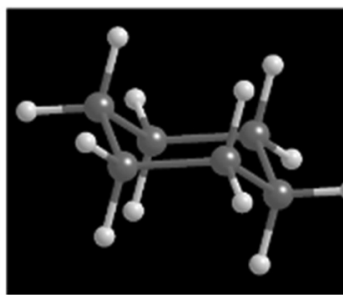

Figure 117. Benzene $\left(\mathrm{C}_{6} \mathrm{H}_{6}\right)$ and cyclohexane $\left(\mathrm{C}_{6} \mathrm{H}_{12}\right)$ molecules. Benzene is a conjugated molecule in which $\pi$ electrons are delocalized over the whole ring (above and below). The benzene molecule is planar. Cyclohexane has only $\sigma$ bonds and is non-planar for this reason.

electronic conduction. It has also been observed that the conductivity of some organic molecules can change with the intensity of an applied electric field.

In the field of inorganic semiconductors, the valence and conduction bands play a preeminent role. The energy difference between the bottom 
of the conduction band and the top of the valence band is the energy gap of the semiconductor. In the field of organic conductors, the important bands are the HOMO (highest occupied molecular band) and the LUMO (lowest unoccupied molecular band). Loosely speaking, the HOMO is similar to the valence band maximum of the inorganic semiconductors and the LUMO is similar to the conduction band minimum.

Carbon nanotubes are macromolecules with high mechanical strength and lengths which are much larger than their transverse dimensions. They have conductivities ranging from metallic to semiconducting depending upon their structure (see chapter 7). Their properties make them a good choice for connections to external electrodes.

Wiring in molecular resistors is easier than molecular wiring to external electrodes. An isolating molecule can be put between two conducting molecules, for instance. Electrically isolating molecules are more common than conducting molecules. Alkanes, which are made of $\sigma$-bonds only are good examples of isolating molecules.

\section{Molecular diodes and transistors}

A molecular diode is a two-terminal device used as a rectifier in electronics. It conducts electricity in one direction only. It is possible to design a molecular diode as indicated in the bottom part of figure 116. It can be a molecule with the donor part separated from an acceptor part by a non-conducting part (spacer) playing the role of a junction barrier. The donor and acceptor parts have delocalized $\pi$-electrons while the spacer has $\sigma$-electrons only. Electrons can go through the spacer by tunneling.

Transistors are 3-terminal devices. They have the ability of amplifying and switching electronic signals. They are needed in molecular electronics to develop the same functionalities as those developed in micro and nanoelectronics. There are basically two ways to develop 3-teminal devices. The first one is to synthesize a molecule with three different ports that can be each connected to three different electrodes.

The second one is to attach a third terminal close to, but not in contact, with a 2-terminal molecule. This third terminal plays the role of a gate and applying a potential to it changes the electrostatic state of the 2-terminal molecule allowing transition from a Coulomb blockade regime to a conducting regime. Such a device has to be operated at very low temperature to avoid any thermal excitation. 


\section{Conductive polymers}

Polymers have been a revolution in the field of materials. They are usually cheap and easy to manufacture. Conventional polymers are good insulators for heat and electricity. Conjugated polymers are a class of polymers that can become conductive when partially oxidized or reduced by doping. They are often named intrinsically conducting polymers. Their conductivity can be that of a metal or that of a semiconductor depending on the nature of the polymer, and its subsequent treatment. For example, the conductivity of polyacetylene can be increased by a factor of $10^{8}$ by oxidative doping with iodine vapor. Doping by chlorine or bromine is also possible. Polyacetylene is a very interesting intrinsically conducting polymer for fundamental studies but is unstable in ambient conditions. It was the first known example of a synthesized conducting polymer. A.J.Heeger, A.MacDiarmid and H.Shirakawa were awarded the Nobel Prize in Chemistry, in 2000, for their discovery of conducting polymers, published in 1977.

The addition of a donor or an acceptor molecule to the polymer is called doping in analogy to techniques used with inorganic semiconductors. Actually, in the case of conjugated polymers, doping induces an oxidation-reduction reaction. In a first step, there is the formation of a cation or anion radical called a soliton or polaron. In a second step, there is either a second electron transfer with the formation of a di-cation or di-anion called a bipolaron, or charged transfer complexes can be formed. Dopants can be electron acceptors such as $\mathrm{Br}_{2}, \mathrm{I}_{2}, \mathrm{H}_{2} \mathrm{SO}_{4}$, $\mathrm{AsF}_{5}$, etc., or electron donors such as alkali metals. Compared to inorganic semiconductors where the concentration of dopants is small (smaller than $1 \%$ ), the concentration of dopants in conductive polymers is large, typically in the range of $20-40 \%$.

The electronic properties of conductive polymers depend to a large part on the value of their bandgap. It is therefore important to be able to adjust this quantity on demand. Several parameters influence the bandgap:

- Increasing the degree of polymerization decreases the value of the bandgap

- The bigger the alternating structure of single and double bonds, the larger is the bandgap

- The presence of aromatic parts in the structures increases the bandgap and stabilizes the polymer.

- It is also possible to modulate the bandgap by playing with the conformation of the monomer molecules or by introducing substituent groups. 


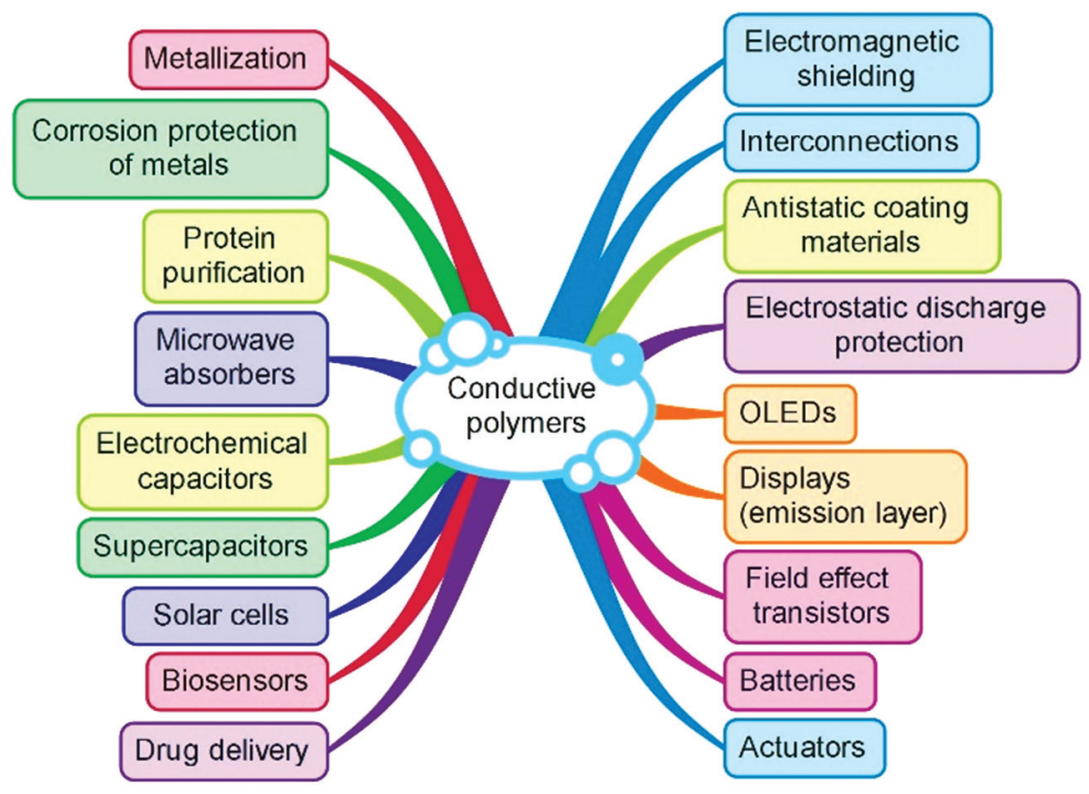

Figure 118. Some applications of conductive polymers.

Several conductive polymers are known: polyacetylene, polyaniline, polypyrrole, polythiophene, etc. Compared to usual polymers, conductive polymers do not possess the same mechanical properties and are not thermoformable. Conductive polymers have several applications that already exist but also a large number of potential applications. Both are shown in figure 118.

As an illustration of some applications:

- It is possible to develop polymer-based photovoltaic devices in which incident photons generate excitons or bound electron-hole pairs in a small molecule layer and in a polymer layer. These excitons dissociate at the small molecule-polymer interface to create free electrons and holes that will generate a current in the device.

- It is possible to manufacture touch sensors, electrochromic pixel displays, biochemical sensors, LEDs or electrical supercapacitors based on conductive polymers.

- Polypyrrole can be used to create electrochemical capacitors. Conductive polymers can also be used in microwave absorbers for radar discretion.

Intermediate between single molecules (monomers) and polymers, oligomers are also often used in molecular electronics. While polymers 


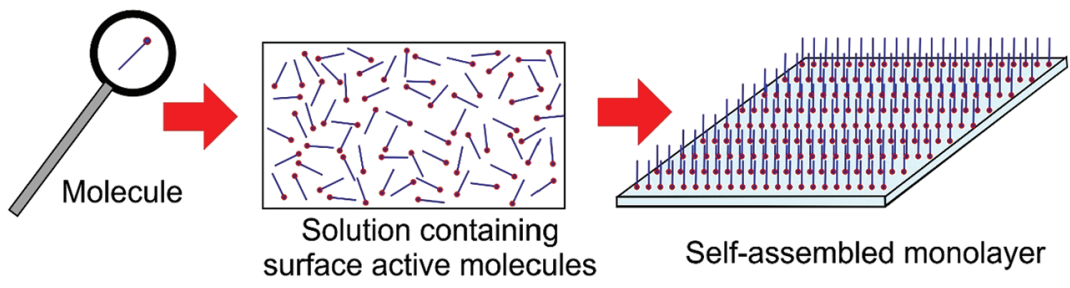

Figure 119. Illustration of self-assembled monolayer deposition via surface active molecules.

consist of a large number of monomer units, oligomers are made of a few monomer units only. They are interesting because they can be synthesized step by step with a complete control of the structure and of their architecture.

\section{Self-assembled monolayers}

Monolayers are important structures useful in many applications. We have already presented the Langmuir-Blodgett method to build monolayers in chapter 6 . There are other techniques to make self-assembled monolayers (called often SAMs). SAMs can be formed by adsorption of a solution or vapor of surface active molecules on a solid surface (figure 119). The molecules spontaneously orientate towards the substrate surface and arrange in an ordered layer corresponding to thermodynamic equilibrium. It turns out that the functional end groups of the initial molecules chemically react with the substrate.

Compared to Langmuir-Blodgett films, where the monolayers correspond to physisorbed bonds, SAMs are more stable because they are based on a strong chemisorption of the head group of the molecules. Self-assembled monolayers open the possibility of making dense functional systems with possible potential applications as switches or memories.

\section{Summary}

Molecular electronics and nanoelectronics are complementary. The former rely on a bottom-up approach while the latter employs a top-down approach. Since nanoscale devices still contain a very large number of single molecules or atoms, it is necessary to use self-organized methods to build objects from single elements at a decent rate. 
Except for special cases such as conductive polymers, for example, molecular electronics is still in its infancy. Even though elementary devices have been made in the laboratory, we are still far from being able to replace the complex micro or nanoelectronic systems that modern society relies upon.

In molecular electronics is that it is possible to have complete control on the molecules forming the building blocks. Furthermore, it is also possible to graft specific functions on a given basic molecule to tailor specific properties of the molecules. These modified molecules are useful as building blocks for the construction of nanoscale devices. Another interest in developing molecular electronics is to better understand the fundamental mechanisms involved in electronic transport processes.

The near future for molecular electronics, and also for nanoelectronics, is that some of the functions developed will become part of larger microdevices. Complex systems will in fact become hybrid systems containing micro, nano and molecular components. 


\section{Nanocatalysis}

Catalysis is a phenomenon in which a small quantity of a substance, called the catalyst, increases substantially the rate of a chemical or a biological reaction without being consumed. Many chemical and biological processes rely on catalysis. The Swedish chemist J.J.Berzelius was the first, in 1835 , to notice that certain chemicals accelerate chemical reactions while remaining unchanged after the reaction. Today, $90 \%$ of all commercial chemical processes rely on catalysis.

\section{Catalysts make life easier}

The principle of catalysis is schematically shown in figure 120 . The initial and final states of a reaction are separated by a potential barrier. The higher and thicker the barrier, the slower is the speed of reaction. The role of a catalyst is to reduce this barrier and make the reaction easier to process.

A catalyst changes the way the reaction is performed, but not a reactant or final product. It modifies the speed of a reaction, its pathway and can guide the reactants towards the production of a specific product.

The lower the activation energy (the height of the barrier in figure 120) the lower is the temperature required for a reaction to occur at a reasonable rate. For example, suppose that a reaction takes place at a temperature of about $1000 \mathrm{~K}$ without a catalyst and that the initial activation energy is $250 \mathrm{~kJ} / \mathrm{mole}$. If a catalyst decreases the activation to $100 \mathrm{~kJ} / \mathrm{mole}$, then the reaction can be carried out around $400 \mathrm{~K}$.

In organic reactions, there are four main types of catalysis used to accelerate chemical reactions: acid catalysis, base catalysis, nucleophilic catalysis and metal-ion catalysis. To illustrate the efficiency of a catalyst in a chemical reaction, we can consider the reaction $\mathrm{C}_{2} \mathrm{H}_{4}+\mathrm{H}_{2} \rightarrow \mathrm{C}_{2} \mathrm{H}_{6}$ in which ethylene is hydrogenated into ethane. Performing this reaction at $300 \mathrm{~K}$ with Platinum as a catalyst enhances the rate of the reaction by a factor $10^{42}$ !

Catalysis can be either homogeneous or heterogeneous (figure 121). In homogeneous catalysis, the reactants and the catalyst are in the same phase. This is not the case in heterogeneous catalysis. The main advantage of heterogeneous catalysis is that the catalyst can be easily used again. Compared to homogeneous catalysis, heterogeneous catalysis is generally cheaper and more selective. However, on a per catalyst atom basis, homogeneous 


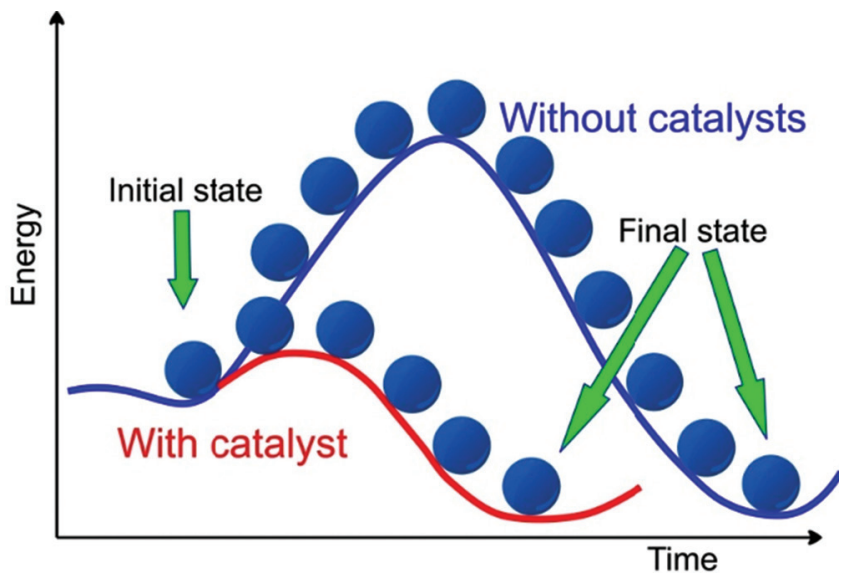

Figure 120. Principle of catalysis.

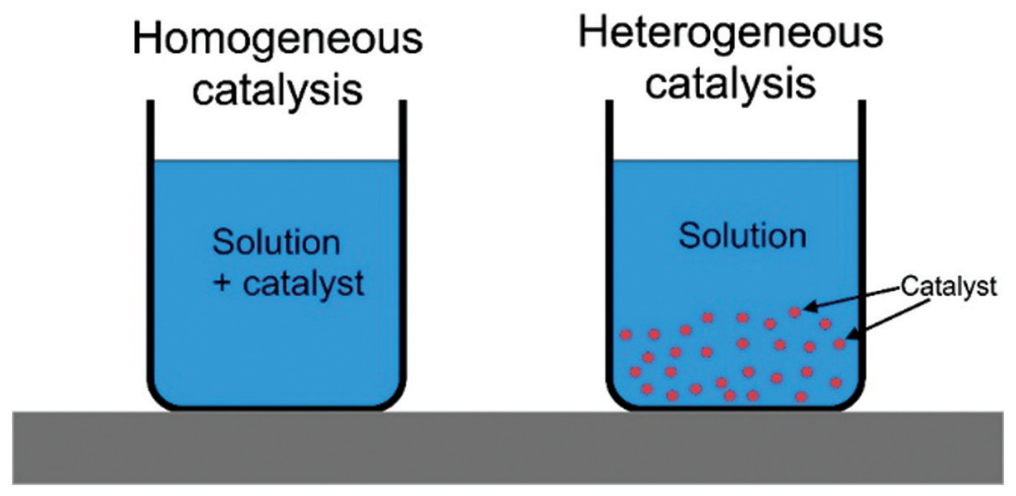

Figure 121. Difference between homogeneous and heterogeneous catalysis.

catalysis is usually more efficient than heterogeneous catalysis. Examples of families of homogeneous catalysts are: acids, bases, metal salts, radical initiators, enzymes. Examples of families of heterogeneous catalysts are: supported metals, solid acids and bases, transition metal oxides and sulfides, immobilized enzymes, polymer bound species.

A good catalyst needs to have several properties which are shown in figure 122 .

It should be highly stable to resist the physical conditions of the reaction and have the possibility to be re-used several times. When several reactions are possible with the reactants, the catalyst should have a high selectivity to favor the desired reaction with respect to the others. While a catalyst cannot change the equilibrium yield of a reaction it can increase the practically achievable yield. An additional desirable feature for a catalyst is that 


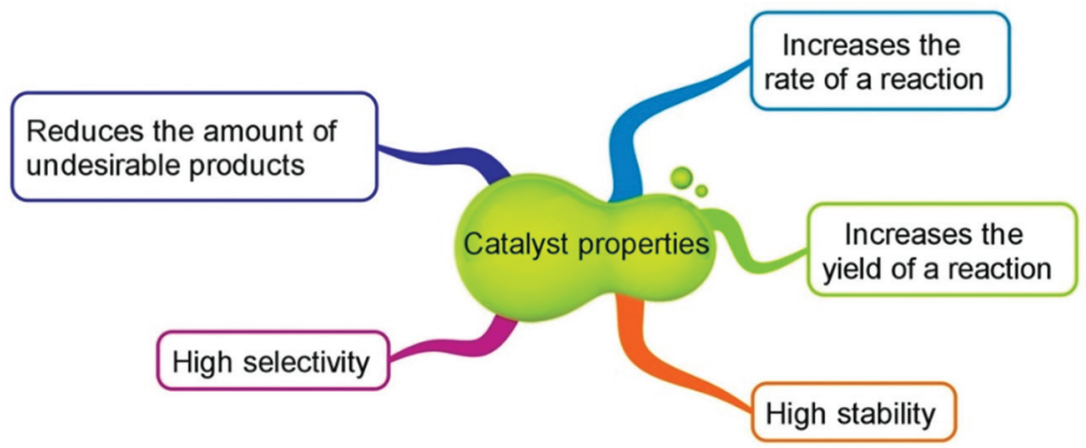

Figure 122. Properties that a good catalyst must have.

it reduces the number of undesirable products formed in the reaction, or helps to produce useful byproducts.

In heterogeneous catalysis, the catalyst is often deposited on a support, especially in the case of metals. The reason is that some metals, such as platinum, are expensive and only the surface is active. Therefore, platinum is deposited on a cheap support which gives also the strength to the whole catalyst system.

The efficiency of a given catalyst depends on several parameters: size, shape, support on which it is deposited... Since the interface between the reactants and the catalyst is one of the most relevant issues, it means that a good catalyst should have a large area of contact. This is precisely what nanoparticles offer, with a high surface area-to-volume ratio, and explains why the nanocatalysis domain is important.

\section{Nanocatalysts for chemical reactions}

Several nanomaterials are now used as catalysts. Some of them are indicated in figure 123 .

In homogeneous catalysis, transition metal nanoparticles are frequently used in the form of colloidal solutions. The solvent used can be water, an organic solvent or a mixture. These solutions must be stabilized in order to prevent aggregation of the nanoparticles otherwise the catalytic activity would drop. The choice of the stabilizer is therefore important to maintain a high reactivity for the catalyst.

The colloidal solution should be made in such a way that the catalyst can be easily recycled. The most frequently used method to synthesize colloidal 


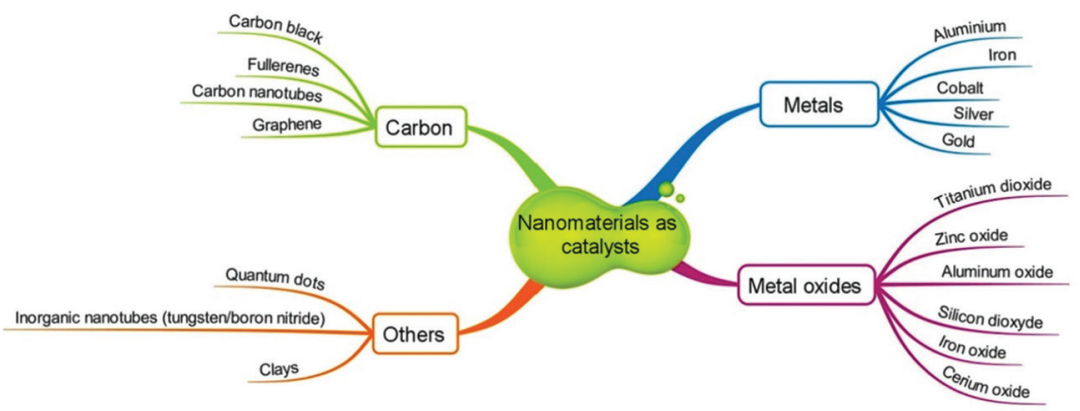

Figure 123. Some example of nanomaterials used as nanocatalysts.

solutions of transition metal nanoparticles is by chemical reduction of a metal salt. There are other synthetic methods that are used less frequently, i. e., thermal, photochemical or sonochemical reduction, ligand reduction, metal vapor synthesis or electrochemical reduction (details can be found at www.nanowerk.com).

In heterogeneous catalysis, the metal nanoparticles are linked onto a support. This requires either a functionalization of the support to adsorb nanoparticles or that the support be manufactured with lithographic techniques.

In addition, nanoparticles can be chemically more active than larger particles. For example, gold is a rather chemically inert metal. It is not corroded under normal external conditions. Surprisingly, it turns out that gold nanoparticles are chemically active and can be used as catalysts. The catalytic properties depend on the gold particle size $(<5 \mathrm{~nm})$, on the nature of the support, and on the contact structure. This last item is important because it governs the availability and order of the gold atoms. Gold nanoparticles are so active that they are able to catalyze carbon monoxide (CO) oxidation at temperatures far below $0^{\circ} \mathrm{C}$. They are also able to decompose gases such as sulfur dioxide which is an air-pollutant.

The Claisen-Schmidt condensation reaction is an aldol condensation of ketones and arylaldehydes to form unsaturated molecules. It is a route to produce molecules such as flavanone and its derivatives which are important intermediates in the synthesis of anticancer, anti-AIDS, anti-inflammatory and antibacterial drugs. This reaction can be catalyzed by $\mathrm{MgO}$. Nanocrystalline magnesium oxide (MgO) is often used as a catalyst ${ }^{11}$. Several crystalline forms are available with different catalytic activity. The less efficient form is commercial Mgo, denoted CM-MgO. It has a specific area ranging 
between 10 and $30 \mathrm{~m}^{2} / \mathrm{g}$. Other crystalline forms can be prepared such as the so-called conventionally prepared $\mathrm{MgO}(\mathrm{NA}-\mathrm{MgO})$ or the aerogel prepared $\mathrm{MgO}$ (NAP-MgO). They have a specific areas of $130-250 \mathrm{~m}^{2} / \mathrm{g}$ and $300-590 \mathrm{~m}^{2} / \mathrm{g}$, respectively. The percentage of ions on the edges, which is related to the reactivity of the catalyst is equal to $0.4 \%$ (CM-MgO), $0.5 \%$ (NA-MgO) and $21 \%$ (NAP-MgO).

It turns out that the yield and speed of the reaction depends very much of the crystalline form of MgO. With CM-MgO, a yield of $85 \%$ is obtained in $5^{0}$ hours. NA-Mgo is more efficient with $97 \%$ in 18 hours. However, the best results re obtained with NAP-MgO: $97 \%$ in 12 hours. Clearly, nanocatalysts are very efficient and their nanostructure plays an important role.

\section{Enzymes speed up biological reactions}

Most biological catalysts are enzymes. The names of enzymes usually end with the suffix "ase" indicating a bond breaking. For example, a peptidase breaks a peptide bond in a protein. An esterase breaks an ester bond, transforming the ester into an acid and an alcohol. The word synthase, on the contrary, means synthesizing instead of breaking. The ATP (adenosine triphosphate, considered as the "energy currency of life") synthase, for example, catalyzes the synthesis of ATP.

There are several models explaining enzyme-substrate binding. Two of them are displayed schematically in figure 124 :

- The lock-and-key model, proposed at the end of the nineteen century by E.Fischer, supposes that there is a perfect fit between the shape of the substrate and that of the enzyme. This is similar to what happens with a key and a lock.

- In the induced-fit model the configuration of the active site changes to fit that of the substrate.

Compared to man-made catalysts used to speed up chemical reactions, enzymes are highly complex molecules. This is illustrated by trypsin, a medium size globular protein that breaks peptide bonds present in other proteins. The mechanism by which the bond breaking occurs is described in the following web site: http://www.bb.iastate.edu/ thorn/BBMB201/ Trypsin_enzymatic_activity.html. The catalytic mechanism takes place at the nanoscale and this is really a nanocatalyst. 

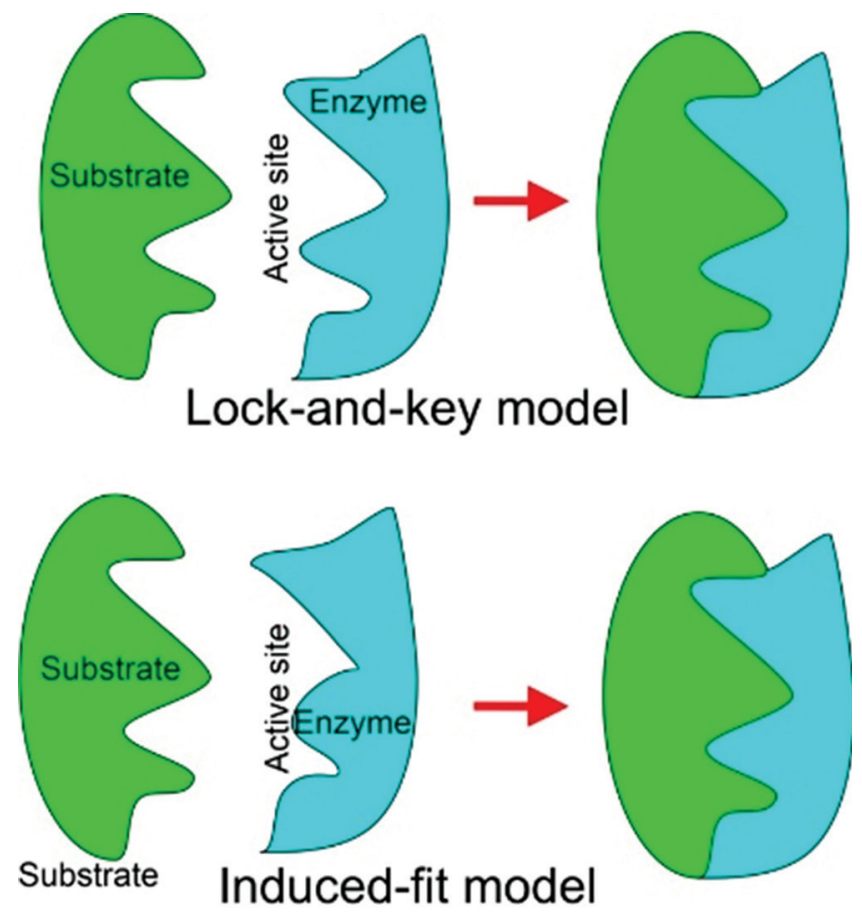

Figure 124. The principle of the lock-and-key and induced-fit models are schematically shown.

\section{Designing nanocatalysts}

Catalytic activity is based not only on chemical formula, but also on structure and the way the catalyst is used. Often, in the past, catalysts have been found experimentally by trial and error methods. Today there is a better understanding of the catalytic mechanisms. Coupling theory and experiment and taking advantage of modern computational capabilities allows today's scientists to develop catalysts faster and more efficiently.

The final goal of catalysis design is to be able to theoretically design new catalysts for chemical or biological applications with catalytic properties better than those already existing. This requires a good understanding of the basic interface mechanism between reactants and the catalyst.

For the simplest catalytic cycle needed to get a chemical or biological reaction to occur one can write:

Reactants + Catalyst $\rightarrow$ Activated Complex

Activated Complex $\rightarrow$ Products + Catalyst 


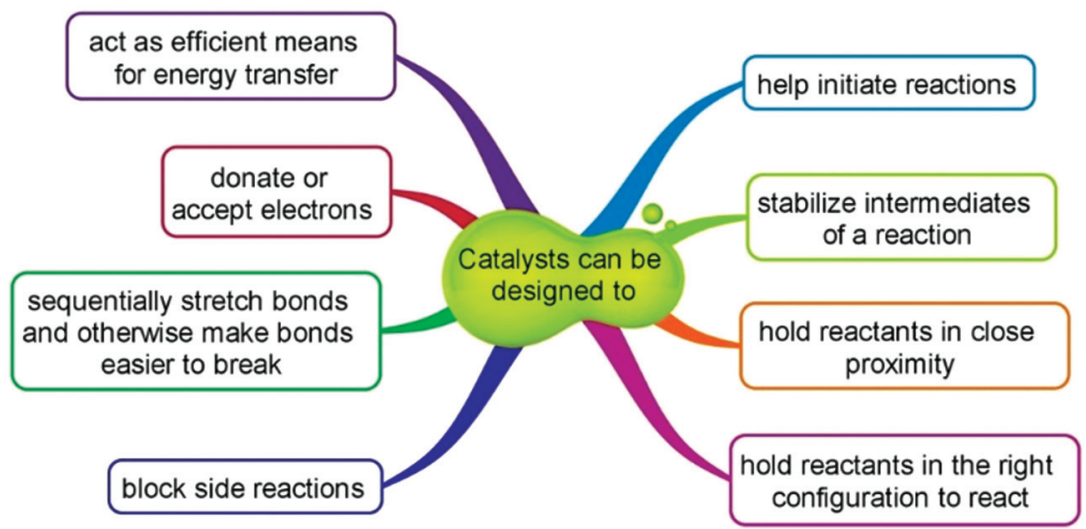

Figure 125. Characteristics of a useful catalyst. Figure based on the presentation of R.I.Mazel, Supplemental Material For Chemical Kinetics and Catalysis, ftp://ftp.wiley.com/sci_tech_med/ chemical_kinetics/.

This shows that the catalyst is not consumed. A good catalyst for a given chemical or biological reaction must have several characteristics. The most important are displayed in figure 125 .

Nanotechnology is at the heart of modern catalysis development. There are several driving forces for developing new nanocatalysts:

- To produce high-value products at low cost with inexpensive raw materials.

- To reduce, as much as possible, the energy needed to produce these products

- To develop environmentally-friendly processes

- To meet more and more stringent environmental regulations such as REACH (Registration, Evaluation, Authorization and Restriction of Chemicals) in the European Union.

- To develop low-cost catalysts replacing catalysts using precious metal

- To favor more efficiently one reaction with respect to unwanted side reactions.

- To produce valuable byproducts rather than waste.

\section{Summary}

Catalysis is an essential domain in the synthesis and transformation industry. A catalyst decreases the activation energy necessary to perform the 
reaction and increases the rate of a reaction. It provides the ability to carry out chemical and biological reactions that would be either impossible or difficult to realize in normal experimental circumstances. Biological mechanisms governing life processes are heavily based on the use of enzymes, natural catalysts. Catalytic activity depends very much on the contact surface between the catalyst and the reactants. For that reason, nanostructuration of a catalyst can be very important. The design and creation of new nanocatalysts offers revolutionary new possibilities. Not only are nanocatalysts more efficient than microcatalysts but they will also open possibilities to facilitate a much wider range of chemical and biological reactions. 


\section{Energy production}

Humans need energy to live. This is provided by food. The basal metabolism of a human being requires about $2.7 \mathrm{kWh} /$ day. Human beings need also energy to produce work, to manufacture objects, to move from one place to the other with vehicles, to produce light when it is dark, to cook food, etc. This energy is provided by various sources such as oil, natural gas, coal, biomass, hydroelectric power, solar power, wind power etc.

The availability of abundant and affordable energy is fundamental to the increasing quality of life enjoyed by the inhabitants of our planet. Since the industrial revolution, among the benefits of this has been a dramatic increase in the life expectancy of people. For example, in France, the life expectancy was less than 30 years in 1880 . It is more than 80 years today. Unfortunately, poor countries still have much smaller life expectancies than rich countries.

The first step in the energy chain is energy production from primary energy sources. At the global level, the primary energy supply amounted to 13.5 $\mathrm{Gtoe}^{12}$ in 2013. Most of the energy that we currently use comes from fossil fuels (oil, coal and natural gas) which are in finite quantities in the earth. Figure 126 shows the percentage of the different energy sources contributing to global primary (prior to any transformation) energy consumption in 2013. Fossil fuels support more than $80 \%$ of the global primary energy consumption.

While Humankind relies heavily on fossil fuels, they are in finite quantity in the earth and will be depleted on a timescale of the order of, at most, a few centuries. This is a serious constraint. Decreasing fossil fuel consumption and replacing it with sustainable energy sources is a high priority at the global level. The relative contribution of fossil fuels to the global primary energy supply has decreased from $86.7 \%$ in 1973 to $81.4 \%$ in 2013 . However, in absolute value the fossil fuel consumption has increased (from about 5,3 Gtoe in 1973 to 13.5 Gtoe in 2013).

A second major constraint facing the world is the emission of carbon dioxide $\left(\mathrm{CO}_{2}\right)$ and more generally of greenhouse gases such as methane, nitrogen oxides, etc. The $\mathrm{CO}_{2}$ emissions increase the greenhouse effect and can induce a climate change with a negative impact on the living conditions on the planet. We are basically emitting about twice as much $\mathrm{CO}_{2}$ as nature can absorb with natural processes.

Final energy corresponds to the energy available to the consumer after transformation of the primary energy (electricity, gasoline, diesel oil, 


\section{Total primary energy supply}

(2013)

\section{Other}

Hydro

Nuclear

\section{Biofuels and waste}

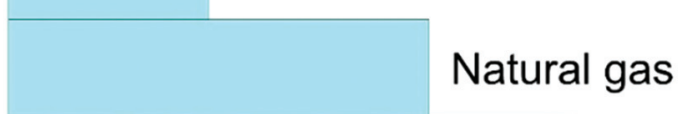

Coal/peat

Oil

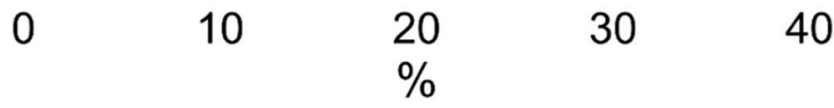

Figure 126. Sources of primary energy consumed, 2013. Other includes wind, solar, geothermal, etc. Data from www.iea.org.

purified natural gas or purified coal, etc.). The global final energy consumption in 2013 was equal to 9,3 Gtoe representing $68.7 \%$ of the total primary energy supply. This inefficiency in the energy conversion is partly due to physical laws, such as the Carnot principle, but also to yields in the different operations. The latter can be increased using innovative technology such as nanotechnology.

\section{Fossil fuels}

Crude oil has to be refined before it can be used by consumers. Gasoline, diesel oil etc. are obtained in this way. The efficiency of the refining processes and the yield are important economic issues. Nanocatalysis plays a key role in this respect since nanotechnology provides the ability to fabricate more efficient catalysts with less active material. In petroleum refining, nanocatalysis is used in the four major processes indicated in figure 127. 


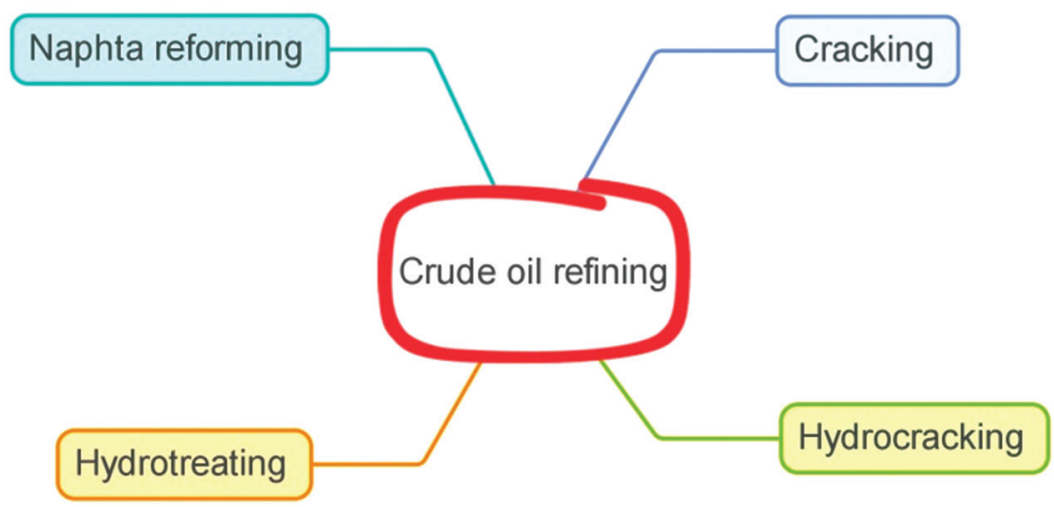

Figure 127. Nanocatalysts are used in four major processes in crude oil refining.

Cracking is the process in which the heavy molecules present in crude oil are broken down into smaller molecules. Hydrocracking is a cracking process in the presence of hydrogen. Hydrocracking is used when catalytic cracking is difficult. In the case of hydrotreating, extra hydrogen is used to remove contaminants. such as sulfur, nitrogen or metals. Naphta reforming is an important application of nanocatalysts because using nanosized platinum particles rather than larger particles greatly increases the efficiency of that process.

Nanocatalysis is also important in the fabrication of synthetic gas (syngas) which is a mixture of carbon monoxide and hydrogen. Syngas can be produced by several methods such as the gasification of coal, or steam reforming of natural gas. Transformations of the syngas are made using four main reactions: water-gas shift, methanation, methanol synthesis and Fischer-Tropsch synthesis.

Using nanocatalysts the water-shift reaction in which the carbon monoxide reacts with water vapor to produce carbon dioxide and hydrogen can be carried out at lower temperatures than with conventional catalysts. This is also true for methanol synthesis. Nanocatalysts are also employed to synthesize methane from the syngas (the methanation process). but the yield decreases as the nanoparticles of the catalysts grow and become larger.

Hydrocarbons can also be synthesized from syngas by means of the Fischer-Tropsch reaction. This process has been used at an industrial scale to synthesize fuel in situations where oil is not available (Germany during World War II, South Africa). Nanocatalysts can provide large increases in efficiency relative to conventional catalyst systems. 


\section{Renewable energies}

Renewable energies are strongly developing globally but their contribution to the global energy consumption remains small. The renewable energy consumption per inhabitant per year has remained almost constant for centuries. It is about 0.2 Gtoe. Biomass is the renewable energy source which is mostly used and hydro power to produce electricity is second. Wind and solar energies are currently swiftly developing because of incentives, but their intermittency is a serious issue. They need either large scale storage facilities or associated gas or coal fired plants to provide electricity when there is not enough wind or sun. Smart grids can help to some extent. So far there is no easy and inexpensive solution to wind or solar energy intermittency.

\section{Solar energy}

Solar energy can be transformed into electricity using photovoltaic cells. They are made with different semiconducting materials. Most of them are made out of silicon either in the crystalline or polycrystalline form. A second generation of cells has been developed based on thin films (cadmium telluride, copper indium selenium diselenide, amorphous silicon). Their efficiencies are smaller but their cost and the energy needed to manufacture them is also smaller. Nanotechnology is already involved in the development of photovoltaic cells and a research effort is underway to develop a third generation of cells with a high-efficiency conversion based on nanostructured materials. In particular, nanocrystal (quantum dots) technologies improve the efficiency of solar cells because they can be tailored to harvest a greater part of the solar energy spectrum. It has been found, in the USA, that PbSe nanoparticles with a diameter smaller than $10 \mathrm{~nm}$ have the ability to generate up to 3 electrons for each absorbed photon. In the solar cells used today, only one electron is produced by an absorbed photon. Multiple electron production can boost the efficiency of photovoltaic solar cells.

Organic photovoltaic cells are also an interesting way to harness solar energy. They are based on dyes. They can be either completely organic or contain mineral components like dye-sensitized photoelectrochemical cells (Grätzel cells). Molecular engineering is essential to find efficient dyes and self-assembly techniques are needed to build the device. Today their 
yields are small and the life times short. Component aging is a great issue in this field but progress is being made.

In all new developments of solar photovoltaic cells, nanotechnology is widely used, either in the form of nanosized objects (nanocrystals, nanoparticles, quantum dots, etc.), or in the nanostructuration of the material. It is possible to manufacture a photoactive nanoscale material which can be printed or painted on to various surfaces. If that is a flexible plastic foil solar cells can be manufactured in rolls. The goal is to provide flexible, low-cost photovoltaic cells that can be manufactured by such simple techniques.

Harvesting solar heat is also a way to harness the energy coming from the sun. Nanostructured mirrors and lenses allow a better solar thermal collection. Aerogels, with nanopores which are both transparent to solar light and good thermal insulators can be used to improve solar collectors. Nanoadditives, nanoparticles or nanopowders can enhance the heat transfer from the collectors to the heat reservoir tank

\section{Wind energy}

Wind energy is harnessed with large wind turbines. The power delivered by a wind turbine is proportional to the cube of the wind velocity. Therefore, the delivered power strongly increases with the wind velocity and the blades are subject to severe mechanical constraints. Wind turbines must even be stopped if the speed of the wind becomes too large (typically larger than $90 \mathrm{~km} / \mathrm{h}$ ). Carbon nanotubes bound in epoxy can be used to make stronger and lighter blades. They can be $50 \%$ lighter than glass fiber blades and the yield of the wind turbine can be increased by $30 \%$.

\section{Energy storage}

Energy storage, and, in particular electricity storage, is the weak point of the energy sector. It is necessary to store widely varying amounts of electrical energy ranging from milliwatt hours for small portable devices, to terawatt hours, for the grid. Smaller energy amounts are also needed for microdevices. The development of intermittent renewable energies requires the development of efficient storage capacities otherwise other sources are needed to produce electricity when there is not enough, wind or sun. 


\section{Batteries}

Among electrical storage devices, rechargeable batteries are particularly important. In a battery, electrical energy is stored in the form of chemical energy. When the battery runs, the chemical energy is transformed into electrical energy through a redox reaction taking place at the electrodes of the battery (except in the case of li-ion batteries where insertion processes take place). The positive electrode captures electrons and the negative electrode releases them. Positive and negative electrodes face each other and are separated by an ionic electrolyte allowing the motion of ions from one electrode to the other.

A battery is usually made of several individual cells associated in series or in parallel to provide the desired voltage and power. The electrodes are porous complex composite systems where nanomaterials and nanostructuration are essential. Carbon black or carbon nanotubes can be used. Nanotechnology is also applied to the development of the electrolyte separating the two electrodes.

Nanotechnology is used in batteries, especially in lithium-ion batteries, to provide increased energy densities with decent recharge times. In Li-ion batteries, the lithium ion $\mathrm{Li}^{+}$is used and at least one electrode is an open crystal structure with empty spaces to intercalate $\mathrm{Li}^{+}$ions. There are several Li-Ion technologies. For example, in $\mathrm{LiCoO}_{2}$ batteries, the oxidation of Co expels the $\mathrm{Li}^{+}$ion which is intercalated in the graphite electrode. During charging, the $\mathrm{Li}^{+}$ion moves from the positive electrode to the negative one in the electrolyte. During the discharge, the opposite mechanism takes place. The main issue is to have a good reversibility in the process taking place at the nanoscale.

Fast charging and discharging is an important quality of a battery which can be obtained if the active materials of the electrodes are thin films. However, this limits the energy capacity of the battery because of the limited amount of active material. Increasing the energy capacity necessitates thicker films and results in slower charging properties. The question of power of the battery is also an issue. Power is related to the ion removal capability which in turns depends on the electrochemical properties of the battery.

\section{Supercapacitors}

Supercapacitors provide another way to store electricity. Their energy densities are intermediate between those of rechargeable batteries and 
electrochemical capacitors. One of their advantages is their high power densities (about 10 times that of a battery) and the fact that they can make a very large number of charge-discharge cycles compared to batteries.

Nanotechnology can be applied to increase the performance of supercapacitors. The power density of a supercapacitor depends especially on the nature and structure of the electrodes. Carbon in different forms is the most frequently used electrode material. Single-walled and multi-walled nanotubes of carbon have the ability to increase the power density of supercapacitors. In the laboratory, an increase of a factor of seven has been measured compared to conventional electrodes.

Construction of supercapacitors with a $3 \mathrm{D}$ nano-architecture in which positive and negative electrodes interpenetrate while being electrically separated from each other is a feasible goal. Paper batteries, combining nanotubes and cellulose layers, have recently been developed in a US laboratory. This opens the way to manufacturing techniques based on rolling and folding.

\section{Electricity}

Electricity is an essential energy vector. The need for electricity is ever increasing. Electricity has to be transported from the producer to the consumer and there are losses. Due to the joule effect: part of the initial energy carried by the grid is lost as heat. The amount of electricity lost in the grid is about $7-10 \%$. The use of high critical temperature superconducting materials at is a way to reduce the losses in part of the grid. Nanotechnology can be used to tailor interfaces and multilayers of the superconducting materials. Presently superconducting cables maintained at the temperature of liquid nitrogen, $77 \mathrm{~K}$, exist and a few commercial installations have been completed worldwide.

Another challenge is to develop a light, low energy loss material to replace the copper cables used today. Carbon nanotubes can meet this demand because they have a better electrical conduction than copper and their weight is about one fifth that of this metal. Furthermore, their mechanical strength is much larger. Positive results for this material have been obtained in the laboratory.

\section{Hydrogen}

Hydrogen is also an energy vector which can play an important role in the future. It can provide an alternative way to store electricity from intermittent 
renewable energies sources such as wind or solar energies. In this case electricity produced by these sources is used to electrolyze water and produce hydrogen. Electrolysis of water is not economically competitive compared to hydrogen production from natural gas by vaporeforming. However, in the case of intermittent energy sources it is a possible solution.

Photoelectrochemical water splitting is another route to produce hydrogen from water. Nanosized semiconductor particles provide the best efficiency to perform this operation.

Hydrogen storage is an important issue. A characteristic of hydrogen is that its energy density per unit of mass is about 3 times that of gasoline. However, in the area of transportation, the key parameter is the energy density per unit of volume and the figure is not as good. Hydrogen stored at 800 bars ( 800 times the atmospheric pressure) has about 5 times less energy density per unit of volume than gasoline. Liquid hydrogen has a similar energy density per unit of volume to that of compressed hydrogen at 800 bars but requires energy to be liquefied from the gas (theoretically, about $4 \mathrm{kWh} / \mathrm{kg}$ are required).

Solid-state storage of hydrogen is an interesting possibility. This can be done using metal hydrides. For example, by forming metal hydrides certain alloys of magnesium have the ability to absorb large quantities of hydrogen. Hydrogen can also be stored by chemisorption or physisorption in carbon nanotubes. Theoretically, single-walled nanotubes can absorb $14 \%$ by weight of hydrogen and multi-walled nanotubes about $7.7 \%$ by weight.

The main issues for hydrogen stored in solid metallic supports are the material loading capacity and the speed at which it is possible to extract hydrogen from the containing matrix. Nanotechnology is of interest in this respect because of the high surface-to-volume ratios existing in nanomaterials. Nanostructured materials, such as activated carbon, can be made extremely porous with tiny cavities which are able to store hydrogen. In all solid-storage solutions, a cycle for charge and discharge of hydrogen is needed.

\section{Fuel cells}

Most fuel cell employ hydrogen as an energy vector. Such cells are used to convert the chemical energy of hydrogen into electricity. The chemical reaction in the fuel cell is basically the inverse of what happens in water electrolysis. Hydrogen reacts with oxygen contained in the air to form water. There are several fuel cell technologies as indicated in figure 128 . 


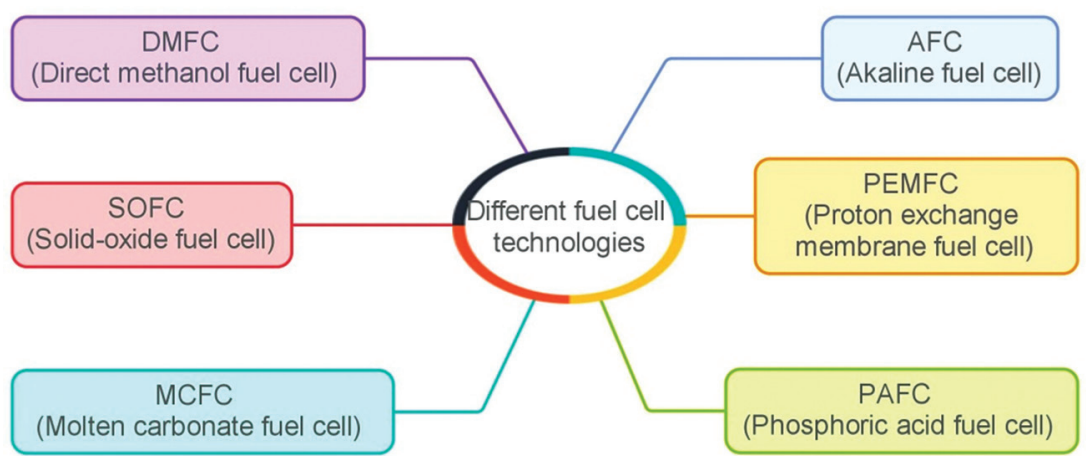

Figure 128. Different fuel cell technologies used to generate electricity.

Except for the DMFC technology using methanol as fuel, other technologies use hydrogen. The most promising ones for large scale applications are the PEMFC for vehicles, and the SOFC for heat and electricity production in buildings. The DMFC technology is interesting for portable devices such as laptop computers or mobile phones. Nanotechnology is essential to progress in the development of fuel cells. Nanostructured materials improve the characteristics of the membranes and electrodes which are crucial parts of fuel cells. Some fuel cell technologies need a catalyst to function. As already noted, nanoparticles provide very large areas in contact with the reactants, increasing catalytic activity. Platinum is widely used but scientists are also trying to find other catalysts that are not noble metals because a large scale deployment of fuel cells could be hampered by a shortage of noble metals. For example, suppose that all cars in world were to be equipped with a PEMFC. With the present quantity of platinum required for the catalyst, a quantity corresponding to almost 300 years of the yearly world production of platinum would be needed.

\section{Thermoelectricity}

Using thermoelectric techniques, temperature differences between two appropriate materials can be exploited to produce electricity and vice versa. There are three kinds of thermoelectric effects:

- The Seebeck effect, discovered in the early 180os, occurs when two different materials in contact are at a different temperature. A voltage difference proportional to the temperature difference is observed. The coefficient of proportionality is called the Seebeck coefficient. The thermocouple is a current application of the Seebeck effect. 
- The Peltier effect is just the contrary. An electrical current flowing between a junction of two appropriate conductors can generate heating or cooling. Cooling is usually the most desired effect for applications.

- The Thomson effect occurs in one material where a thermal gradient is present. If an electrical current is applied, heat can be produced. If a heat flux exists, an electrical current is produced.

Currently, part of sun's radiation can be converted to electricity using photovoltaic cells. This corresponds to about $58 \%$ of the radiated energy (the UV and visible part of the light spectrum). The remaining part, $42 \%$, is in the infrared region (heat). Harvesting this infrared part is also desirable. This is possible employing the Seebeck effect. The efficiency of conversion depends on the nature of the materials used to make the junction. Semiconducting materials are particularly useful because it is possible to tune their electrical properties. Developing hybrid systems containing photovoltaic and thermoelectric cells delivers the ability to harness more energy from the sun.

Apart from solar thermoelectric conversion, thermoelectric devices are used in several niche applications such as producing electricity in deep -space missions, in regions where they do not receive much energy from the sun. The radioactive isotope, ${ }^{238} \mathrm{Pu}$, is used as a thermal energy source and SiGe as the thermoelectric junction. There are also applications such as those used to warm or cool seats in luxury cars.

If small amounts of energy are needed (wrist watches, pacemakers...) the small differences of temperature within the body, or between the body and the ambient temperature, is enough to operate a thermoelectric device.

The efficiency of thermoelectric devices is around $7 \%-8 \%$. A much better efficiency, in the range of $15-20 \%$ would make them useful in large scale applications. Using nanomaterials and nanostructuration, new thermoelectric devices can be developed. For example, energy could be recovered from the exhaust pipe of a car. In research employing nanostructured materials an efficiency increase of the order of $50 \%$ has been obtained.

\section{Nuclear energy}

Both the fission of heavy elements and the fusion of light elements can release large amounts of energy. The technology of the former is well in 
hand and nuclear fission reactors provide about $5 \%$ of our global primary energy. Fusion power is still in the research stage.

In current nuclear plants a large variety of materials are involved. But it is in the reactor vessel that materials are submitted to the most extreme conditions of temperature, pressure, radiation, chemical and mechanical stresses. Outside the reactor vessel there is, at a much lower level, also a degrading influence of radiation on concrete, cables, the corrosion of heat exchangers, etc.

Consider the materials used inside the reactor vessel, for example the cladding of the nuclear fuel. That fuel is enclosed in a leak tight housing. Basically, as the nuclear fuel is burned, the nature of the chemical composition changes and a few gaseous elements are produced. All the products must remain confined inside the housing in order not to contaminate the coolant flowing around the housing. The housing is submitted to high temperatures, high neutron radiation, and the physical transformation in the burned fuel induces strong mechanical constraints on the housing materials. This is amplified when a high burn up (i.e. when a lot of energy is extracted from the nuclear fuel) is demanded.

Mechanical and plastic properties of materials, such as metals or alloys, depend on the mobility of the linear defects (dislocations) in those materials. These dislocations impact the elasticity limit of the material and its overall mechanical behavior. In nanostructured materials, the increased number of interfaces can anchors dislocation and increase the strength of the material. Furthermore, the interfaces facilitate recombination of point defects created by particle irradiation. There are several ways to amplify the number of interfaces: added or precipitated nanoparticles, nanostructuration, thin films, superlattices, etc.

Nanostructured materials can be used to push the usage limits of conventional materials. This is useful to prepare the next generation of nuclear reactors based on fast neutrons. Some of them use sodium as a coolant and operate at temperatures in the range of $400-650^{\circ} \mathrm{C}$ under a pressure of a few bars. In a fast sodium nuclear reactor, the fissile fuel is in the form of pellets of mixed uranium dioxide and plutonium dioxide piled up in leaktight pins placed in sub-assemblies of stainless steel housing which are cooled by a sodium flow. New steels reinforced with oxides have been developed to suit these extreme operating conditions. They are called ODS-steels (oxide dispersion strengthened). ODS are metallic materials reinforced by nanosized oxide nanoparticles manufactured by powder metallurgy. This nanostructuration substantially improves the mechanical properties (breaking strength under tension, creeping, thermal stability). However, 
the anisotropy of the mechanical properties of ODS might prevent their industrial development. On the other hand, interesting results have been obtained with nanolayer systems possessing selfhealing properties. This technology is useful for the manufacture of "accident tolerant nuclear fuels" because they exhibit better radiation resistance during normal operation.

The development of high temperature gas reactors is another line of research. These are fast neutron reactors cooled by a gas (helium). They are operated at temperatures in the range of $500-85^{\circ} \mathrm{C}$, providing a good yield to produce electricity because of the Carnot principle. The fuel is the form of carbide and the cladding of nuclear fuel requires materials resistant at these very high temperatures. It is necessary to develop new ceramic materials to fulfill this requirement but this is still at an early research stage. At any rate, nanostructuration is essential. Silicon carbide is a possibility and, in particular, long fibers of $\mathrm{SiC}$ can be used to give good ductile properties.

Nanomaterials and nanostructured materials can also be expected to make important contributions in future nuclear fusion devices.

\section{Summary}

Nanomaterials, nanoparticles and nanostructuration play a role in many areas of energy production. They can substantially improve technologies based on conventional materials and processes. Nanotechnology will be essential in the development of future of solar cells. Nanocatalysts will continue to play a key role in the transformation processes converting primary energy sources to final energy sources available to the consumer. Energy storage capabilities, the weak link of the energy domain, will be improved using nanotechnology in the area of batteries, supercapacitors, etc. Fuel cells that use hydrogen to produce electricity will use nanotechnology to develop better membranes and catalysts. Finally, harnessing additional energy production techniques such as thermoelectricity will rely on nanotechnology to reach an industrial stage. Nanotechnology is expected to transform the energy domain over the next few decades. 


\section{Housing}

The provision and maintenance of adequate housing is a major part of our modern economy. In the United States, for example, housing and housing services account for about 18 percent of the Gross Domestic Product. About 30 percent of the annual income of a typical household in the US is spent on housing. For lower income households the percentage can be significantly higher. In the European Community, an average of about 21 percent of household income is spent on housing. Construction costs, upkeep and repairs, and energy consumption account for the bulk of housing expenses. Nanotechnology can be employed to improve or replace conventional materials and technologies and reduce long term housing expenditures.

\section{Outside the building}

The primary materials used in construction are wood, cement, concrete, steel and glass. The construction sector is typically rather conservative and is strongly oriented towards low-technology, cost-efficient solutions. Current uses of nanotechnology are mostly targeted to improve surface durability but nanostructuration of bulk materials offers a potential of improved structures and reduced costs.

For example, current chemical treatments for preserving wood could be enhanced or replaced by employing nanosized particles as preservatives or carriers of preservatives. Such nanoparticles could also be used to improve dimensional stability and durability of wood or wood laminates and to improve bonding with paints and adhesives. An array of nanosensors built into wood could serve to monitor environmental stresses or biodegradation.

Portland cement, often used in construction, consists of a mixture of limestone, clay and gypsum. Chemical reactions take place when water is added and mixed with the cement and other possible additives. After a slow drying, a hard and durable material is obtained. Concrete is a mixture of cement, sand, rock, water and small amounts of other additives Concrete is the most commonly used man-made material on earth. About 14 billion tons are used each year. This corresponds in the average to about 2 tons per inhabitant of the earth. Considerable effort has been devoted to find chemical additives and admixtures that improve the physical and mechanical characteristics of concrete. Fly ash or silica fume (amorphous 
$\mathrm{SiO}_{2}$ ) are often used to strengthen concrete. In ancient times volcanic ash was used. What produces the high strength of today's concrete is its crystal structure in the nanometer range. This strongly depends on the chemical reactions occurring during the setting and hardening process.

Concrete is a nanostructured multiphase composite material containing amorphous phases, tiny crystals and bound water. There are three main regions at the nanoscale which are important and where nanoengineering can play a role: solid phases, liquid phases and the interfaces between liquid-liquid and solid-solid. In addition, extra nanosized material can be included in the concrete to give it specific properties. Addition of nanosized particles can improve the mechanical properties of concrete. Nanoparticles of silica and of titanium dioxide are the most used additives in nanomodified concrete. Other tailored nanomodifications can alter the cement paste, reducing the permeability and increasing the durability of concrete. $\mathrm{SiO}_{2}$ nanoparticles in small quantities uniformly dispersed in the cement paste promote and accelerate cement hydration. Compressive and flexural strengths of cement mortars are notably increased if $\mathrm{SiO}_{2}$ and $\mathrm{Fe}_{2} \mathrm{O}_{3}$ nanoparticles are included compared to conventional mortars. Furthermore, better characteristics are obtained compared to the case in which microparticles of silica fume are used. The goal is to understand and control what happens at these scales to optimize the properties of concrete in terms of performance, volume change and durability.

Traditionally steel reinforcement is used in concrete but it is also possible to use other textiles or fibers. Carbon nanotubes or nanofibers are also interesting because they improve the strength and other mechanical properties of concrete. Including carbon nanotubes leads to very high tensile strength and tougher concrete. The added concentration has to be very low (typically $0.025 \%$ by weight of cement. Because of the potential toxicity of these nanotubes, acceptable safety standards for their use are essential.

Steel plays a major role in the construction industry. The structural failure of steel is due to cyclic loading. Frequently fatigue occurs at discontinuities such as bends, grooves, holes or defects such as scratches. These are often referred to as stress risers. Nanotechnology can improve the physical properties of steel. The addition of copper nanoparticles can limit the number of stress risers. The use of vanadium and molybdenum nanoparticles can improve the delayed fracture problems associated with high strength bolts. Carbon nanotubes can be used to strengthen steel cables.

Another important material in construction is glass. Nanocoatings are used to make self cleaning glass. Titanium dioxide $\left(\mathrm{TiO}_{2}\right)$ nanoparticles 
used for this purpose catalyze reactions that break down organic dirt. $\mathrm{TiO}_{2}$ is hydrophilic. Rainwater which hits the glass spreads evenly instead of forming droplets. This facilitates the removal of the dirt. Nanocoatings can be used also to reduce light and heat transmission through exterior windows. Light-or heat responsive smart coatings, or electrically conductive window coatings with transmission properties that are altered on demand when a voltage is applied, may be used to control the inner environments of buildings and reduce energy costs. Multifunctional smart windows that incorporate various features are under development.

Nanotechnology can also be used to improve functionality of the exteriors of buildings. Technical textiles embedding nanomaterials can provide better thermal insulation. Smart textiles embedding nano- and micro-sensors can monitor the energy consumption of the house and detect early damages to the structure. Inorganic nanoparticles can be homogeneously incorporated in polymers for façade coating. Nanocomposite coatings provide paints which are resistant to dirt, free of cracks and the color remains the same over time. Nanoceramics or ceramics coated with nanofilms can provide new capabilities such as self-cleaning.

\section{Inside the buildings}

A primary goal of sustainable development in housing is to reduce the amount of energy needed to live in the house. We have already noted above the role of smart windows incorporating nanotechnology in reducing heat transmission. Other areas in which nanomaterials can be used to reduce energy demand are described in the following subsections.

\section{Insulation}

Maintaining a year around comfortable temperature level in our buildings usually requires a combination of heating and cooling capabilities and appropriate insulation. Insulation is normally done passively using materials that reduce heat transfer or leakage. The transfer of heat from one point of space to another occurs by three different phenomena: conduction in which there is a flow of thermal energy but not of matter, convection where there is a flow of matter during the heat transfer, and radiation in which electromagnetic waves transfer the thermal energy. Radiation is the way we get energy from the sun. 
A large part of the transfer of thermal energy in buildings takes place by conduction or convection. In this case, the best insulator is vacuum. This is the principle of the double walled vacuum vessels we call thermos bottles. Maintaining large volume vacuum containers for a long period of time is an expensive technology and is not generally practical for buildings.

Air is a good insulator provided it stays motionless. Insulators based on air are manufactured in such a way that the air trapped inside tiny volumes remains confined. In the domain of nanostructured materials, nanocellular foams are good thermal insulators because each of the large number of nanopores trap air molecules. Nanoporous materials are very good thermal insulators and can be used in smaller thicknesses than traditional insulators. For example, compared to expanded polystyrene, nanoporous materials can offer the same thermal insulation in a volume 20 times smaller.

Aerogels are nanoporous materials in which the liquid has been replaced with gas. Sometimes called "frozen smoke", they can contain $95 \%$ air. Silica aerogel can be made with a density of $1 \mathrm{~kg} / \mathrm{m}^{3}$. This density is the density of the aerogel calculated without the air inside. Without contained air it would float in the air since the density of air is greater, $1.2 \mathrm{~kg} / \mathrm{m}^{3}$. Nanogels are extremely light, have a good light transparency (up to $75 \%$ of transmittance) and can support 2,00o times their own weight. The most common aerogels are brittle, have a poor humidity resistance and are expensive. Organic aerogels are less common but have better insulating properties than inorganic aerogels. Attempts are being made to manufacture hybrid organic-inorganic aerogels.

Radiation insulation is used to block infrared radiation coming from the sun. With nanofilms, it is possible to almost completely block infrared and UV radiation while letting visible light pass through. This reduces air conditioning energy consumption. Furthermore, it is possible to have active films, whose reflectivity can be controlled on demand, either manually or automatically using sensors. A ceramic-aluminum nanocoating, applied to cars reduces the thermal energy passing into the car by about $40 \%$ and has the additional property of being corrosion-resistant.

\section{Lighting}

Lighting accounts for about $19 \%$ of the world's electricity consumption. In 2005, the annual electricity consumption for lighting was equal to 3,400 TWh. The distribution among different sectors is shown in figure 129. 


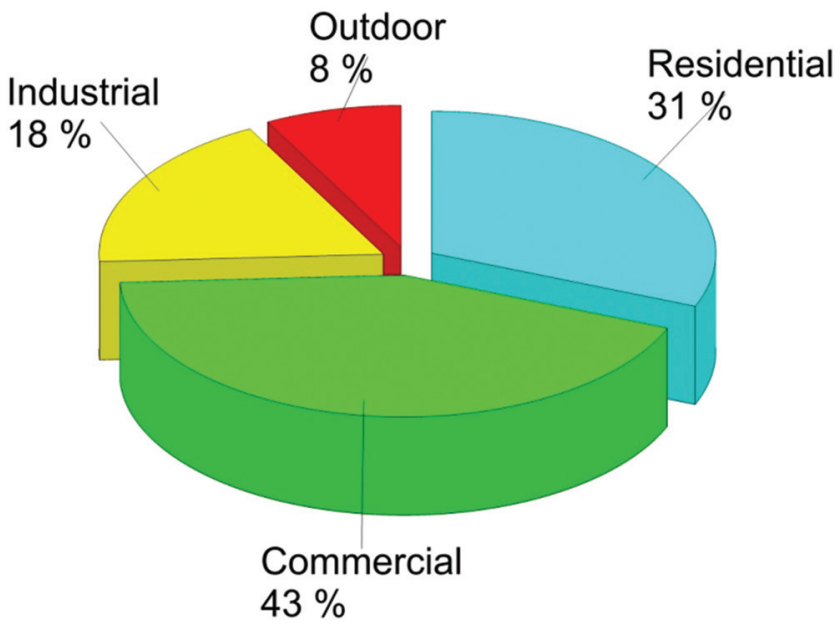

Figure 129. World electricity consumption for lighting by sector in 2005. Data from www.iea.org.

The amount of energy transformed into light is very small compared to the initial amount of energy consumed. However, the efficiency has increased constantly over the ages. In terms of luminous flux, a candle gives 0.2-0.4 lumens per watt and an incandescent light bulb about 7-15 lumens per watt. The latter corresponds to an efficiency of about $5 \%$. Incandescent bulbs have a lifetime of the order 1,00o hours. Halogen lamps have longer lifetimes, around 2,000-3,000 hours, and better efficiencies, about 15-27 lumens. Compact fluorescent lamps have efficiencies around $20 \%$ (of the order of 45-75 lumens per watt) and fluorescent tubes are even more efficient. Their lifetimes are of the order of 6,000-7,00o hours. However, compact fluorescent lamps have a serious environmental drawback because they each contain about $5 \mathrm{mg}$ of mercury. Mercury is a highly toxic poison and these lamps should not be released into the environment. They demand special treatment when they are discarded.

The future of lighting is very likely light emitting diodes (LED). Their efficiencies increase continuously, thanks in particular to nanotechnology. They are still expensive, typically 2 to 5 times greater than other lighting solutions with similar performances. However, prices are now going down and they will soon be highly competitive. Their performances vary according to the technology and manufacturing processes. LEDs can have a lifetime of the order of 20,000-40,000 hours and a luminous flux of 90100 lumens per watt. They can be switched on and off or dimmed without affecting their lifetimes. The electricity consumption of the most recent LEDs is an order of magnitude less than incandescent lamps. Thus it is possible to significantly reduce the consumption of electricity devoted to 
lighting. In countries in which a large part of the electricity is generated from fossil fuels, $\mathrm{CO}_{2}$ emission could be greatly reduced.

Inorganic LEDs, which are the most common today, are basically III- $V^{13}$ semiconductor devices. They have a high constantly improving efficiency and a high brightness. Inorganic LEDs often use quantum wells in the active region. These quantum wells consist of few nanometer layers of a specific material, such as InGaN, sandwiched between thicker layers of doped GaN. It is possible to design LEDs emitting different colors.

Organic LEDs is a field in development. They are based on organic semiconductors. Both OLEDs, made of small organic molecules, and PLEDs (polymer light emitting diodes), which use polymers in the emissive layer, can be made. The color of the light can be tuned by the structure of the emitting molecules present in the OLED. The advantage of OLEDs is that they can be thin and flexible. Large areas can be manufactured. They are currently used in smartphones. But organic LEDs still lag behind inorganic LEDs. Inorganic LEDs provide an intense tightly focused light source whereas OLEDs generate a more diffuse light.

Figure 130 shows a schematic drawing of an OLED and figure 131 of a double heterostruture OLED. OLED displays contain a matrix with a large number of single OLEDs. The addressing of individual OLEDs can be either passive-matrix (POLEDs) addressing or active-matrix addressing (AMOLEDs). AMOLEDs are present in several portable devices such as smartphones and tablets.

The use of OLEDs has seen a swift development. They were introduced into the small display market (e. g. cell phones) in 2006-2007. They appeared in medium and large size displays such as TVs or laptops around 2008. OLEDs were first used in lighting applications in 2010 and in the flexible display market in 2012.

Nanotechnology contributes to the LED domain by increasing performance while decreasing costs.

\section{Heating, ventilating and air conditioning}

Aluminum components are very common in heating systems. The combustion of oil or natural gas produces small amounts of corrosive residues, in particular because of traces of sulphur in the fuel, which attack aluminum surfaces and decrease the efficiency of a heating system. Nanoparticle coatings for the aluminum prevent corrosion and the heating system becomes more efficient: it requires less fuel and $\mathrm{CO}_{2}$ emissions decrease. 


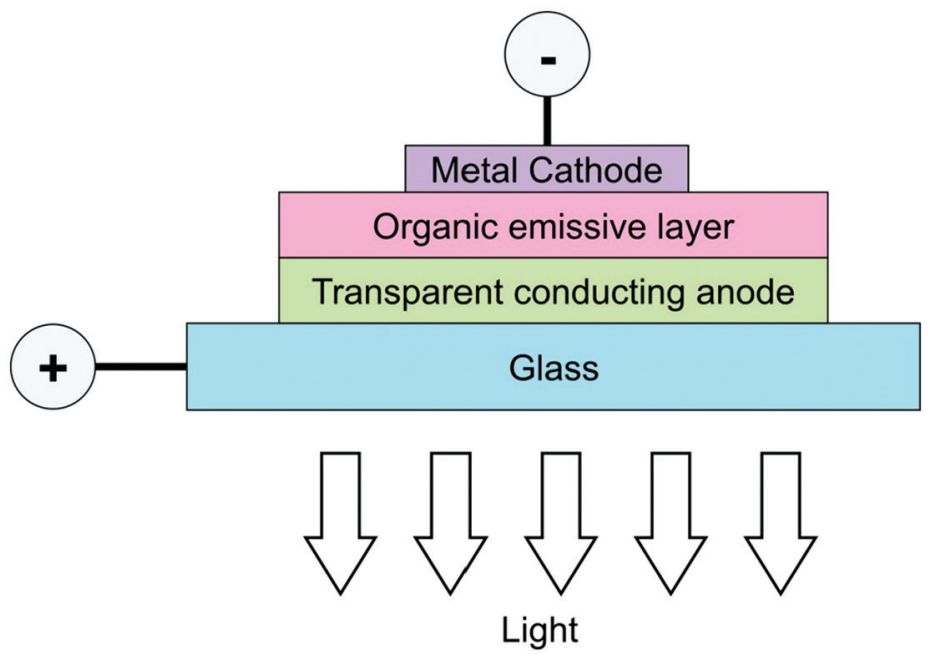

Figure 130. Schematic drawing of an OLED. The organic emissive layer is sandwiched between two conductive layers.

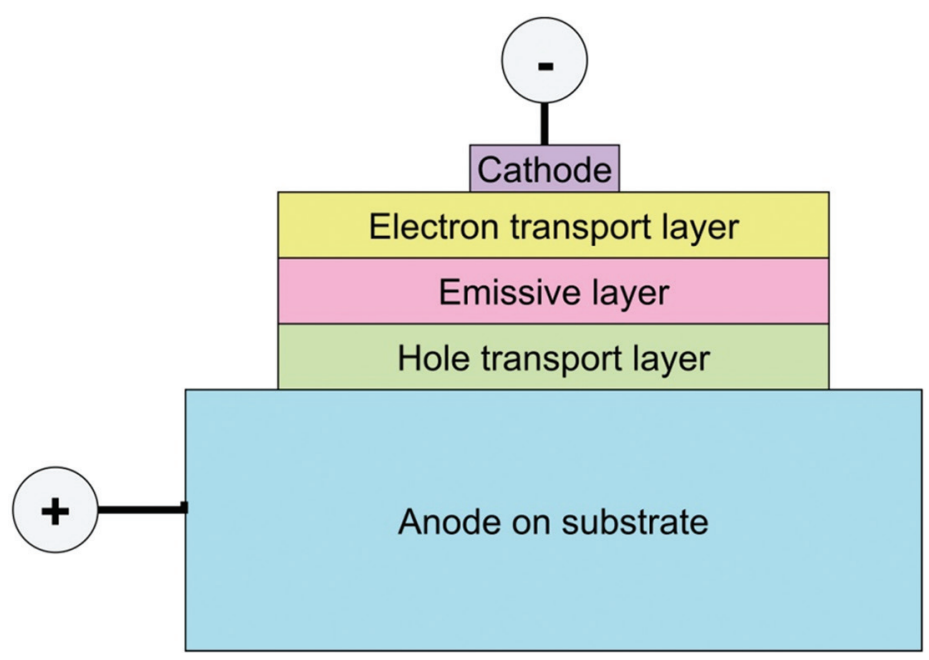

Figure 131. Schematic structure of a double heterostructure OLED. It consists of an organic emissive layer sandwiched by an electron transport layer and a hole transport layer.

With corrosion protection the aluminum can be used in heat exchangers. Nanocoated heat exchangers can reduce the amount of oil required during the heating period by about $10-15 \%$. 
Nanotechnology has the ability to make breakthroughs in the heating, ventilating and air conditioning (HVAC) area. For example, NanoAir units, developed by the Dais Analytic Corporation (www.daisanalytic.com) can be used to heat, cool, dehumidify or humidify air. They rely on proprietary nanomaterials and do not need CFC/HCFC refrigerants which contribute to the greenhouse effect and destruction of the ozone layer. In this application, nanoparticles are used to harness energy in such a way that there is a separate control of moisture and temperature.

\section{Summary}

Nanotechnology has already spawned a number of applications in housing. Nanoadditives notably improve the properties of concrete. Textiles with embedded nanomaterials can be used for outer covering of buildings. Selfcleaning external surfaces and glasses can be produced. Inside the buildings, nanoporous materials can insulate buildings with better efficiencies than conventional materials. LEDS and OLEDs have created a revolution in lighting technologies. Improved HVAC systems have higher efficiencies. 


\section{Nanotechnology in automobiles}

Modern civilizations rely heavily upon motorized vehicles to transport both people and goods. The transport of people from one point to the other all over the world is a major part of the global economy and of the modern lifestyle. The rapid growth in the number of automobiles, first observed in developed countries, and now in developing countries reflects both the desire and the necessity for convenient and flexible on-demand transportation.

In the automotive area, as in others, nanotechnology is applied to produce materials with novel structural and functional properties tailored to a particular need. These materials are used to improve safety and reliability, to reduce weight without sacrificing structural integrity, to reduce energy consumption, to reduce cost, etc. Improvements in comfort and driving pleasure are also important aspects to which drivers and passengers are responsive.

Some of the areas in which nanotechnology offers the possibility of improvement are shown in figure 132. Safety, environmental protection and comfort are the main areas where nanotechnology can have a large impact.

Safety is of course the most important point. Each year, about 1.3 million people in the world die in car or truck accidents. About 50 million are injured. This figure is similar to the population of South Korea.

It is also important to note that road transportation relies heavily on fuels to propel our vehicles. Half of the oil extracted in the world is used to propel vehicles and more than $95 \%$ of the fuel used in road vehicles is derived from oil. Burning oil, in the form of gasoline or diesel, leads to the emission of carbon dioxide $\left(\mathrm{CO}_{2}\right)$, particles, unburned hydrocarbons and carbon monoxide (CO). These emissions can have serious negative effect on the environment and human health. Decreasing fuel consumption by filtering or treating the gases emitted from the exhaust pipe can reduce this pollution. Nanostructured filters and nanocatalysts can help in this respect.

Nanotechnology is already important in the automotive industry. Examples in Figure 133 identify current applications of nanotechnology in many parts of a car.

\section{Bodywork}

Fuel consumption and safety can be improved using nanomaterials. For that it is necessary to reduce the weight of the vehicle while maintaining or improving the performance of the affected components. 


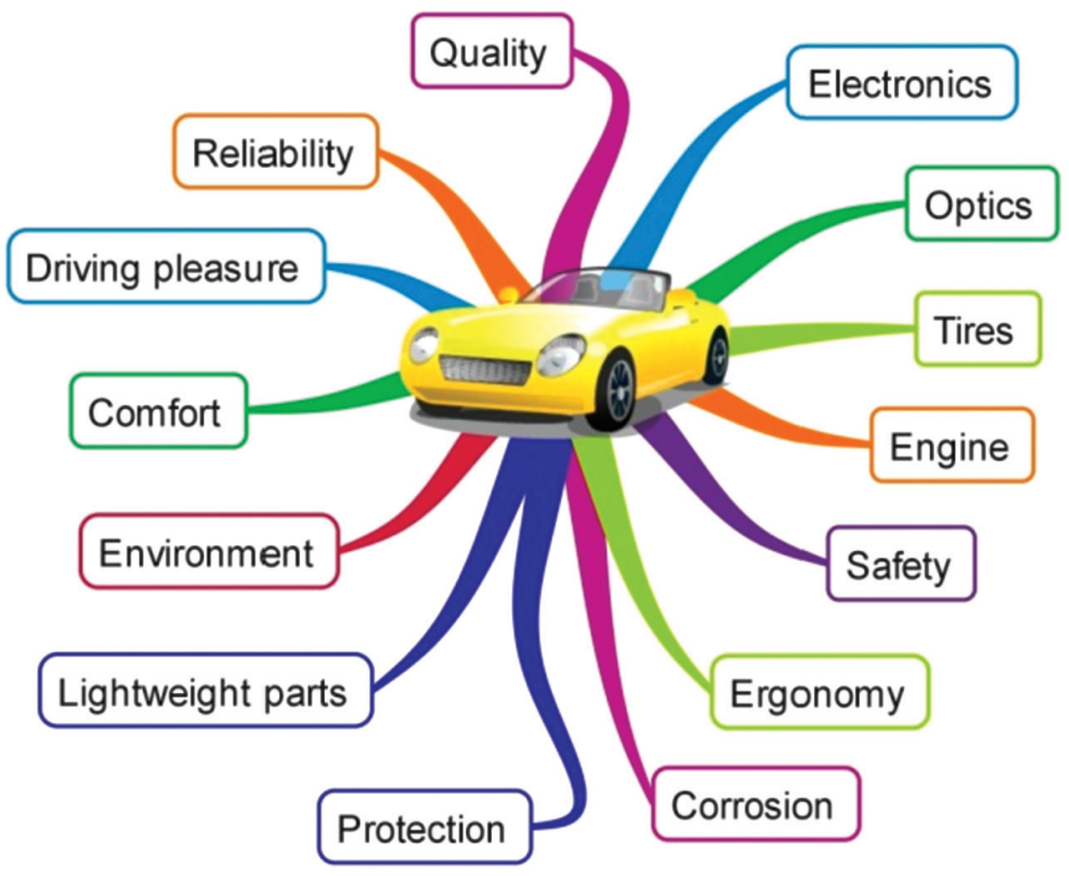

Figure 132. Different areas of the automotive domain that nanotechnology can improve

High-strength flexible parts could be used as crash-absorbers reducing the impact of a collision. Nanotechnology is already employed to manufacture high-strength steel using 5-10 $\mathrm{nm}$ embedded metallic carbon nitride particles. This steel is fatigue-resistant and permits the manufacturing of lighter parts. The development of other fatigue-free nanosteels is envisaged in the near future.

Another important issue is corrosion protection. Galvanic coating processes using chromium VI are now forbidden because they are dangerous for human health and the environment. Chromium III protection processes are far less efficient. Using a three-layer protection: zinc, chrome III and a surface coating of silica nanoparticles of, provides outstanding protection against corrosion and, in addition, provides self-healing properties against small damages to the surface.

In the future, part of the body of the car will be covered with enhanced self-healing materials based on nanotechnologies. Micro-encapsulated healing molecules and a polymerization catalyst put into a common matrix, but separated from each other will be used. Any damage which cracks the surface will break the microcapsules. The healing agent they contain 


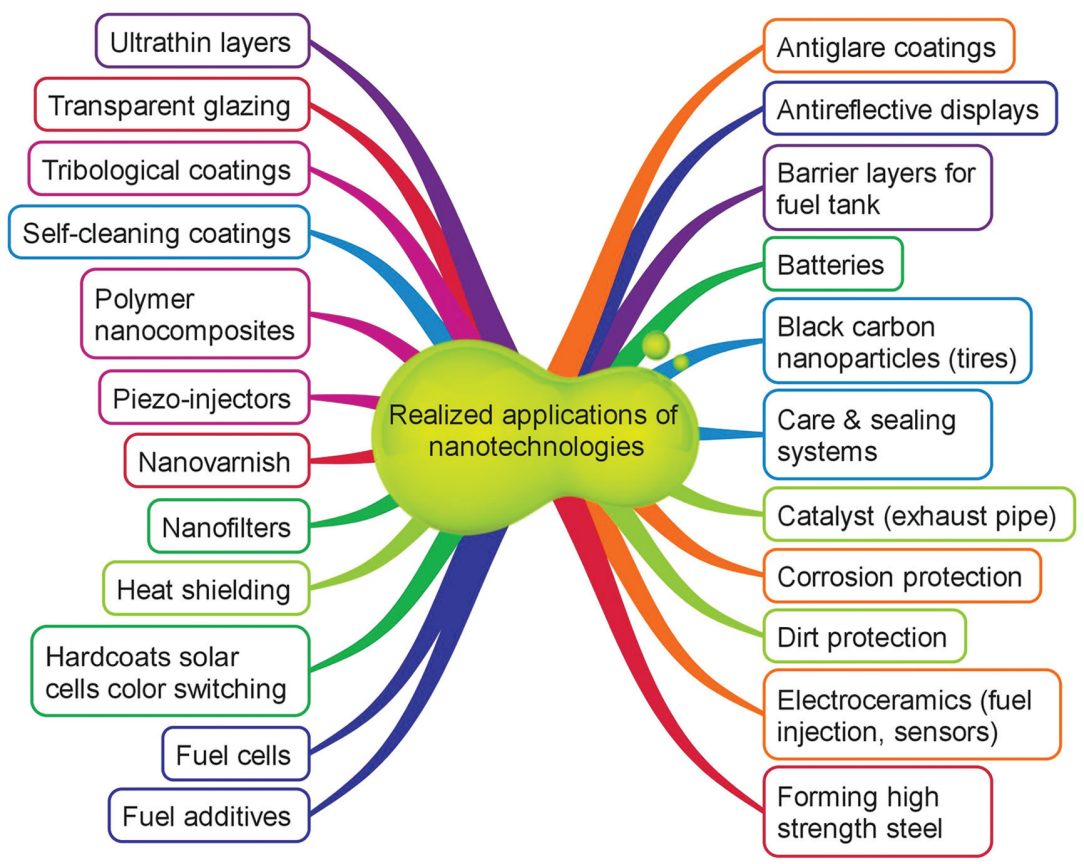

Figure 133. Examples of current applications of nanotechnology in the automotive domain.

will be released and fill the crack. This technique is exemplified by the work of White and al. schematically illustrated in figure 134 .

In the past soldering or bolting was extensively used to build a car. Gluing, increasingly being used in the automotive industry, provides the advantages of low cost and high-processing speeds. Gluing often requires warming the two components to temperatures of the order of $180^{\circ} \mathrm{C}$. This can induce adverse thermal stresses in the components. Using glues containing nanoferrites, it is possible to trigger the gluing process by microwave radiation at wave lengths in which the interaction occurs with the glue only. This requires less energy than conventional gluing and is faster. Consequently the thermal stress to the components is substantially decreased. This technique is called "gluing on demand". Another useful technology for repair is to use a glue which has the ability to detach (unglue) on command. Biologically inspired materials based on the Gecko effect discussed in chapter 5 can provide the "stick and detach on demand" capability. These materials are nanostructured.

The body of a car represents a large surface exposed to external conditions. Using nanotechnology, it is possible to improve several properties or characteristics of the body. By functionalizing the surface with specific 


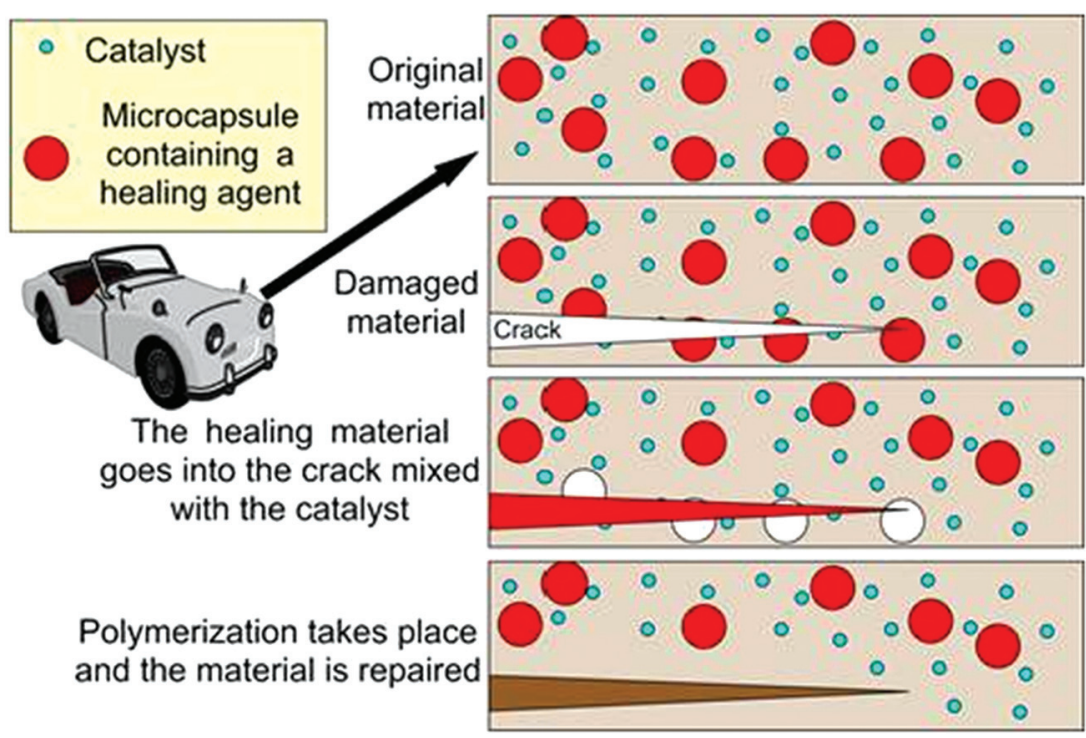

Figure 134. The use of a microencapsulated healing agent embedded in a structural composite matrix containing a catalyst capable of polymerizing that healing agent makes it possible to repair a small crack occurring in the body of a car. During crack formation, the microcapsules are ruptured, releasing healing agent into the crack by capillary action. In contact with the catalyst, the healing agent polymerizes and the crack is repaired.

molecules it is possible to adjust the wetting properties or to protect the surface against external stresses. Silica nanoparticles with a diameter ranging from 7 to $40 \mathrm{~nm}$ used in car paints can make the paint very hard as the paint dries because of a crosslinking mechanism between the nanoparticles. It is 3 times more resistant to scratches. The color of the paint becomes more brilliant. A nanovarnish containing ceramic nanoparticles deposited on the paint can further increase the scratch resistance and maintain the brilliance for a longer time.

Cars have a large area of glass and plastics, windows, mirrors and headlights. Nanostructuration of their surfaces at the nanoscale provides the ability to create surfaces with a gradient in the refractive index. This technology produces optical surfaces with reduced reflection and improved transmission. A good quality reflecting coating is obtained with a nanometer-thickness aluminum oxide layer. Lenses for headlight covers are usually made of polycarbonate which is an extremely hard material and is lightweight. However, it has poor scratch resistance. Coating polycarbonate with paints containing aluminum oxide nanoparticles produces a high scratch resistance, while retaining high optical transparency. It is also possible to have a hydrophobic and oleophobic treatments, based on 
nanometer-thickness layers, to make it easier to clean dirt, fingerprints, sweat deposited on the surface. Self-cleaning surfaces are interesting for glazing, wing mirrors, headlights, alloy wheels, bumpers, etc.

Nanotechnology can be used to produce electrochromic windows whose optical properties change with an applied voltage. This is useful to maintain interior temperatures or for reducing glare from the rear-view mirror of a car at night. This increases safety and comfort of the vehicle for the driver.

It is difficult to keep a car clean. The body and interior have to be cleaned regularly. Anti-dirt products based on nanoparticles are now available. Water and fat repellent nanoparticles deposited on metal, fabrics, glass or plastics can prevent dirt, water or fats from sticking to their surfaces.

\section{Interior of the car}

Using nanomaterials or nanostructured materials mimicking the Gecko effect, it is possible to manufacture fabrics which are dirt-resistant because of their hydrophobic and oleophobic surfaces. These surfaces are self-cleaning, a property interesting for car seats. Photocatalytic materials, such as $\mathrm{TiO}_{2}$ nanoparticles, can decompose dirt. Nanotextile materials have an important future in cars by notably improving the quality of conventional fabrics made of natural or synthetic fibers. Nanocapsules containing a fragrance can be embedded in the fabrics of seats. The fragrance is progressively liberated at each mechanical stress.

Nanofiltration can be used in two applications. The first is control allergenic particles, pollutants or odors. For that nanofilters made of nanofibers are more efficient than conventional filters. Nanofilters are also useful to reduce pollutants emitted by the exhaust pipe. Nanocatalysts, providing much larger areas in contact with the chemical products to be treated, transform the pollutant emitted in the exhaust pipe into harmless molecules.

\section{Chassis and tires}

Tires must reconcile opposite properties: they need to have a good road grip, but a low-rolling resistance; they should be resistant against abrasion, but prevent the car from sliding. Tires based on natural rubber have architectures and additives that give them the good properties currently manifested. They are much different than the tires made in the past. Additives 
such as carbon black, silica, or organosilane nanoparticles are essential to produce tires of excellent quality. The sizes and geometries of the nanoparticles used have a great influence on the final properties of the tire. For example, the introduction of carbon black nanoparticles has improved abrasion resistance and increased the lifetime of a tire by a factor of five. Typically, the thickness of a tire decreases about $5 \mathrm{~mm}$ after $50,000 \mathrm{~km}$ of driving. In the nineties, silica nanoparticles were introduced into the tire manufacturing process. They have significantly increased wear resistance.

The viscosity of a dilatant material increases with the rate of the shear strain. This kind of material, called a shear thickening fluid, is an example of a fluid in which conventional laws of hydrodynamics fail to apply. For that reason, they belong to a wider class of fluids called non-Newtonian fluids. Yoghurt is a common example of non-Newtonian fluid: its viscosity depends on the way you have mixed it with a spoon. A dilatant material can be a colloid containing nanoparticles, which are organized in ordering layers thanks to the Van der Waals interactions. If the shear forces happen to be more important than the Van der Waals forces, flocculation occurs and the fluid behaves more like a solid and is highly viscous. The viscosity of a dilatant fluid changes because the shear stress induces a phase transition from an ordered state to a disordered state so the material looks more like a solid. Dilatant fluids made of a dispersion of nanoparticles can have several applications in the automotive area. They can be used as damping systems or for shock absorption. They can be used in all-wheel-drive coupling, in pedestrian protection, etc.

\section{Power train}

Nanotechnology is also present in many parts of the power train. The aim is to produce more energy-efficient, more environmental-friendly, safer and more comfortable vehicles.

Nanocrystalline piezo-injectors can be used in diesel vehicles, allowing control of the injection valve with an accuracy in the nanometer range. Friction of the pistons of cylinders of the engine waste energy and reducing this friction is an important goal. A nanocrystalline material is able to do that providing the additional advantage of decreasing abrasion and increasing the lifetime.

More generally, nanocoatings are of interest because they are wear-resistant and lead to hardened surfaces. They are particularly useful for highly stressed components such as bearings, cylinders or valves of the powertrain. 
Removing waste heat of the vehicle powertrain is still an important issue because of a regular increase of thermal power density. Increasing the heat exchange surface of a vehicle radiator is not desirable because it increases the weight and the size of the device. Therefore, it is necessary to look at new coolants and thermal coupling materials such as nanofluids. A nanofluid is a liquid containing dispersed and suspended particles with sizes in the nanometer range. It has been shown that an inclusion of these nanoparticles increases the thermal conductivity. Studies are also underway to improve thermal and rheological properties of lubricants by adding nanoparticles.

\section{Electronics}

Electronics has become of ever increasing importance in our vehicles. Electronics accounted for about $7 \%$ of a car's value in 1970, $20 \%$ in 2005 and $40 \%$ in 2010. Most of electronics used today are based on microprocessors etched with dimensions smaller than $100 \mathrm{~nm}$. Electronic sensors are important to maintain a proper functioning of the internal combustion engine. Electronic components made of nanolayers of semiconducting materials are used for engine control, but also in other parts of the car such as airbags, anti-lock brakes and stability control of the vehicle.

European Community legislation requires the electronic industries to eliminate lead from solder, which was traditionally an alloy of tin and lead $(\mathrm{Sn}-\mathrm{Pb})$. New alloys have been developed. One new alloy is an eutectic made of tin, silver and copper. It requires temperatures around $235^{\circ} \mathrm{C}-245^{\circ} \mathrm{C}$, that is about $35^{\circ} \mathrm{C}$ higher than for $\mathrm{Sn}-\mathrm{Pb}\left(210^{\circ} \mathrm{C}\right)$. The higher temperature of operation induces more stress on the electronic components being assembled. Therefore, efforts are being made to reduce the operating temperature. A promising research track appears to be the addition of nanoparticles. The melting points of metals decrease as the sizes of the nanoparticles decreases. Drops of tens of degrees can be obtained using nanoparticles with sizes below $10 \mathrm{~nm}$.

\section{Batteries and supercapacitors}

Batteries can store energy. The development of plug-in hybrid vehicles and electric vehicles requires having reliable batteries with high energy densities per unit of mass. Improvement of batteries is obtained using 
Capacitor discharged

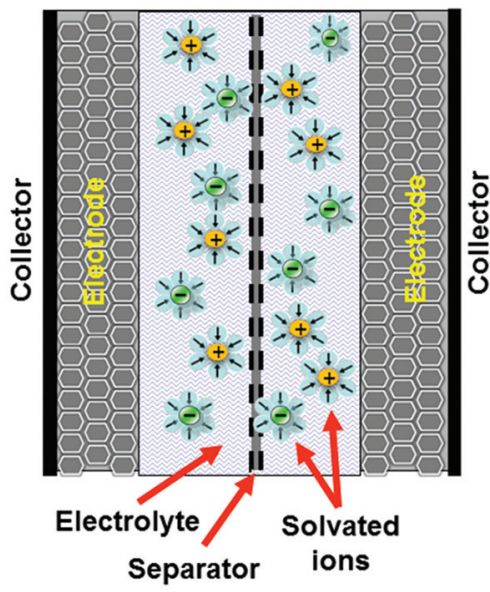

Random distribution of ions

\section{Capacitor charged}

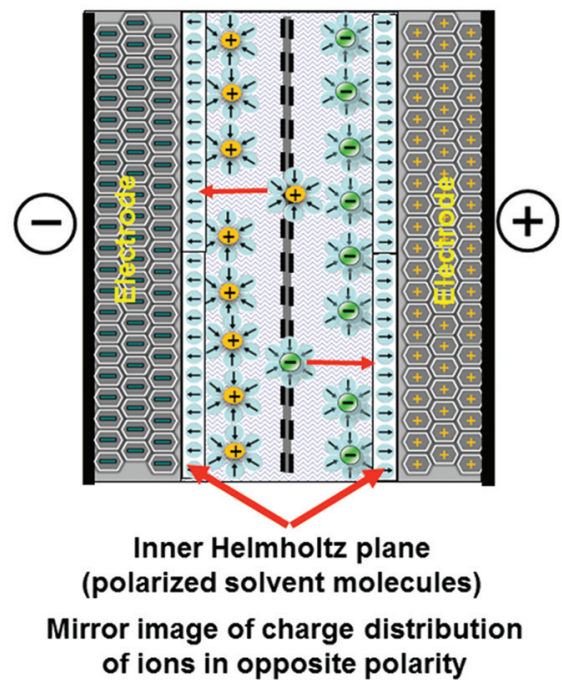

Figure 135. Distribution of ions in a discharged/charged supercapacitor. Image from Wikimedia Commons (http://commons.wikimedia.org). Author Elcap.

nanocomposite plastic to produce solid polymer electrolytes, but also by nanostructuring the electrodes. Typically, a car needs about $15 \mathrm{kWh}$ to travel $100 \mathrm{~km}$ and lithium-ion batteries used in electric vehicles have an energy density of about $120 \mathrm{Wh} / \mathrm{kg}$. The smaller energy density of Li-Ion batteries dedicated to vehicles compared to those that are manufactured for portable devices such as cell phones, laptops, etc. which have around 200 $\mathrm{Wh} / \mathrm{kg}$, is for safety reasons. Indeed, in the case of overloading, the Li-Ion technology used for portable devices can often lead to accidents or damage to the battery. Considering the large amount of energy stored in the battery of a car, similar accidents would have serious consequences.

Supercapacitors can also be used in electric and plug-in hybrid vehicles. They store a small amount of energy but are able to deliver a large amount of power. For that reason, they are interesting to provide fast acceleration to a vehicle. Compared to batteries, they can make a very large number of charge-discharge cycles (more than 100,000). The principle of a supercapacitor is shown in figure 135 .

Nanomaterials and nanostructuration are needed in the development of supercapacitors. Indeed, a very large effective surface is required to get a high capacity and this can be provided by highly porous layers. 


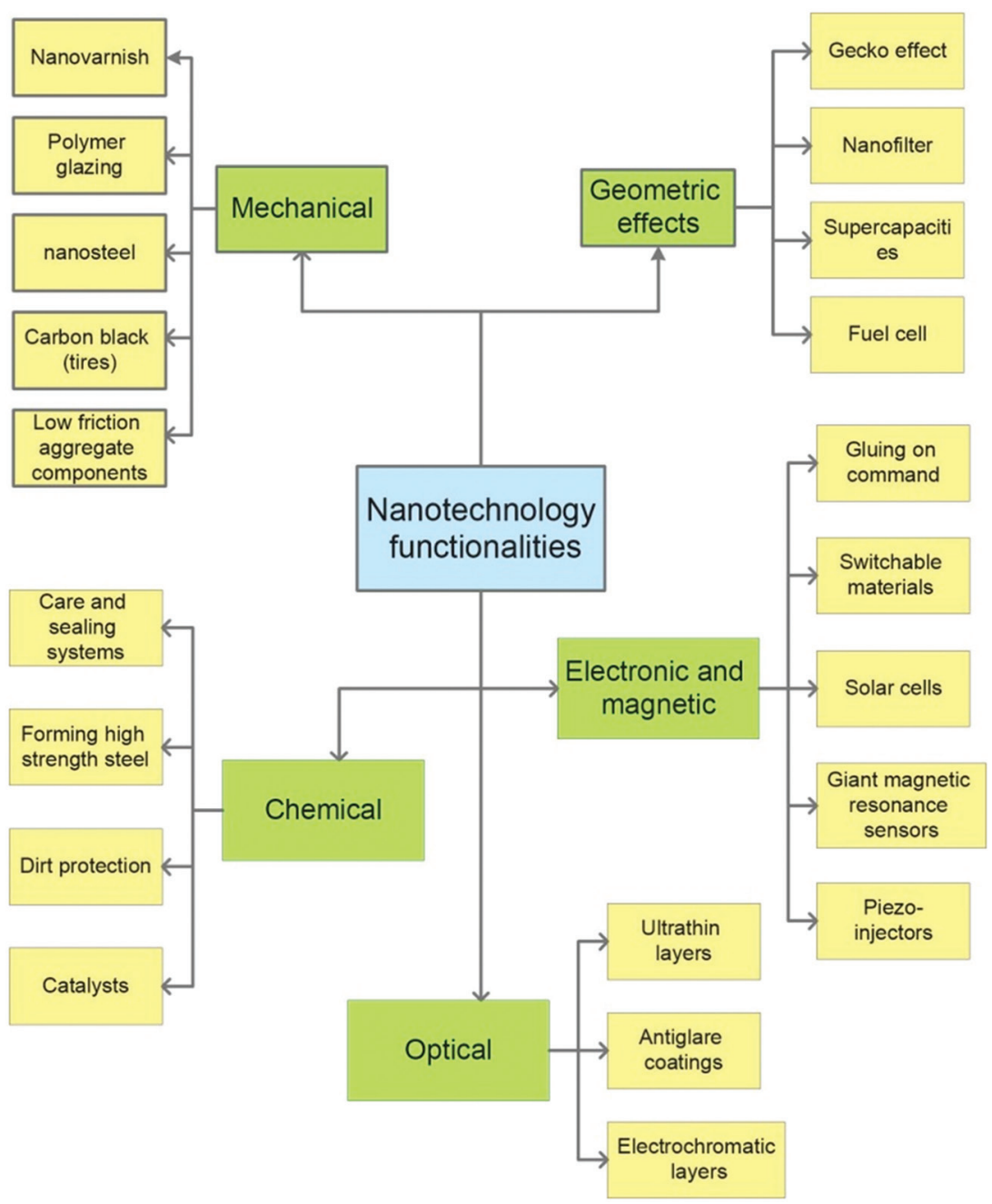

Figure 136. Applications of nanotechnology in the automotive sector. Built from the report "Nanotechnologies in automobiles". Innovation potential in Hessen for the automotive industry and its subcontractors, http://www.hessen-nanotech.de.

Nanotechnology will play an even more important role in the next generation batteries or supercapacitors and in fuel cells of the future vehicles to be fueled by hydrogen, etc.

\section{Summary}

Each second, about 13 cars are manufactured in the world. This corresponded, in 2011, to the manufacture of 76 million cars. There are now more 
than one billion vehicles in the world and the automotive industry represents a large part of the world economy.

Figure 136 summarizes areas of nanotechnology either already existing or under investigation for use in automobiles. 


\section{Defense and security}

Defending and maintaining the security of a country and its population are critically important endeavors. These activities, pursued by both the military and civilian authorities of a country encompass a broad range of activities including anticipation, detection and response to threats to the safety of a country and its citizens. As in other domains, nanotechnology can make valuable contributions, either by improving existing technologies or by introducing new ones. Apart from its direct contributions to defense and security activities, the development of nanotechnology contributes to maintaining a country's technological parity with respect to other countries.

\section{Defense}

For the past several decades, sophisticated technological developments in the areas of defense and security have become increasingly reliant on dual-use technologies that can be used in both military and civilian applications. This evolution reflects the fact that research on and development of, these technologies are extremely expensive. The vast and diverse capabilities in the civilian domain insure rather rapid progress and economy of scale for civilian associated technologies. Research and maintenance costs can be reduced by using commercially developed components in defense and security applications, where possible. Information et communication technologies already rely heavily on nanotechnology since the integrated circuits are designed with an accuracy that is now in the range 10-30 $\mathrm{nm}$

Defense activities benefit strongly from applications of four main areas of nanotechnology. (figure 137).

A few results of applying nanotechnology to defense needs are:

- Helmet sensors and wireless networks providing information and communication applications for soldiers.

- Hybrid power and nanocatalysts for energy applications in military vehicles

- Self-healing structures and biomimic lightweight materials for aeronautics and targeted drug delivery techniques for soldiers derived from life science research. 


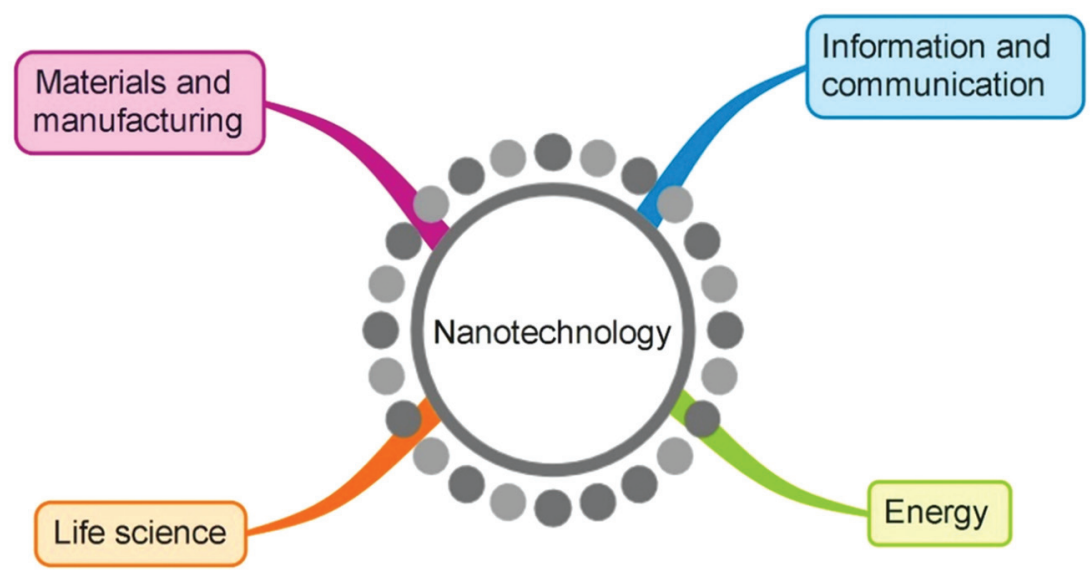

Figure 137. Four main areas of nanotechnology utilized for defense.

- High strength nanomaterials for satellites and adaptive structures for planes or missiles.

- Microelectronics systems which are now designed at the nanoscale and present in all information and communication systems.

- Lower weight portable weapons

- Guns that detect, target and fire automatically with self-guided bullets.

\section{The modern warrior}

Military equipment is evolving rapidly. The equipment of today's warrior is very different from that used during World War II or the Vietnam war. That of future warriors will be even more advanced. These warriors will be connected in real time with other soldiers and with their logistical support units. This will give a group of soldiers a shared intelligence making their action more efficient. Furthermore, this will allow continuous monitoring of the health and mental state of each soldier.

Figure 138 shows areas where nanotechnology can contribute to the equipment of the future soldier.

The evolution of battle gear is towards a lightweight, comfortable battle suit with temperature and moisture control that embeds high-technology capabilities and provides better protection against various projectiles. By high technology we mean sensors and electronic devices that allow communication, that monitor health and that can react immediately to chemical or biological agents. The battle suit can be made ultrahydrophobic by depositing nanolayers of teflon. 


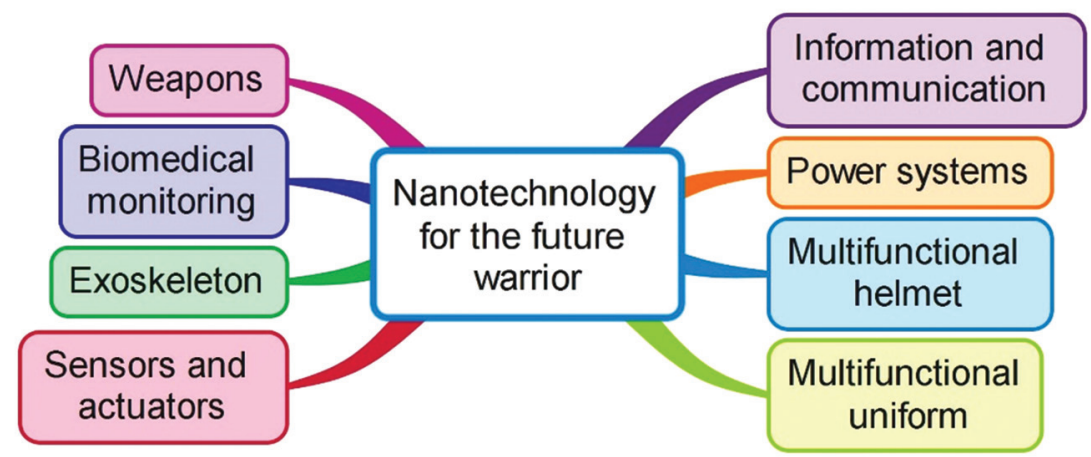

Figure 138. Nanotechnology is involved in providing many components of the equipment of the future soldier.

Protection against bullet damage or stabbing can be provided by "liquid body armor" consisting of a Kevlar vest containing a shear thickening fluid made of nanoparticles suspended in a liquid. Under normal conditions the fluid is easily deformable. But, upon impact, the fluid stiffens and undergoes a transition to a rigid material that prevents the projectile from penetrating into the body of the soldier. The force of impact of the bullet is distributed over a large area reducing the injury due to the kinetic energy of the projectile.

Nanofabrics can be designed for active camouflage in analogy to that existing in nature such as that of the cuttlefish for example. Active camouflage is based on panels and coatings that change appearance depending on the surroundings. The ultimate goal is to create an invisibility cloak. Research in this direction is based on negative-index metamaterials that we have already discussed.

As far as life sciences is concerned, nanotechnology will facilitate heath treatment and body repair. Smart biocompatible implants will sense the health status of the soldier and actuate targeted drug delivery by functionalized nanoparticles in case of health problems. Techniques for regenerative medicine for wound healing and, to some extent, to repair or rebuild organs and other parts of the body are also under development.

Since the equipment of the soldier will become more and more sophisticated and the soldier will also carry sophisticated weapons, minimization of weight is also an important issue. In addition, to power high tech portable equipment requires appropriate energy sources. Several solutions using nanotechnology continue to be investigated: lightweight energy sources such as batteries, flexible solar cells, devices to harvest vibrational energy, or thermal energy for low power applications. Micro fuel cells and micro nuclear batteries are also among the possibilities. 


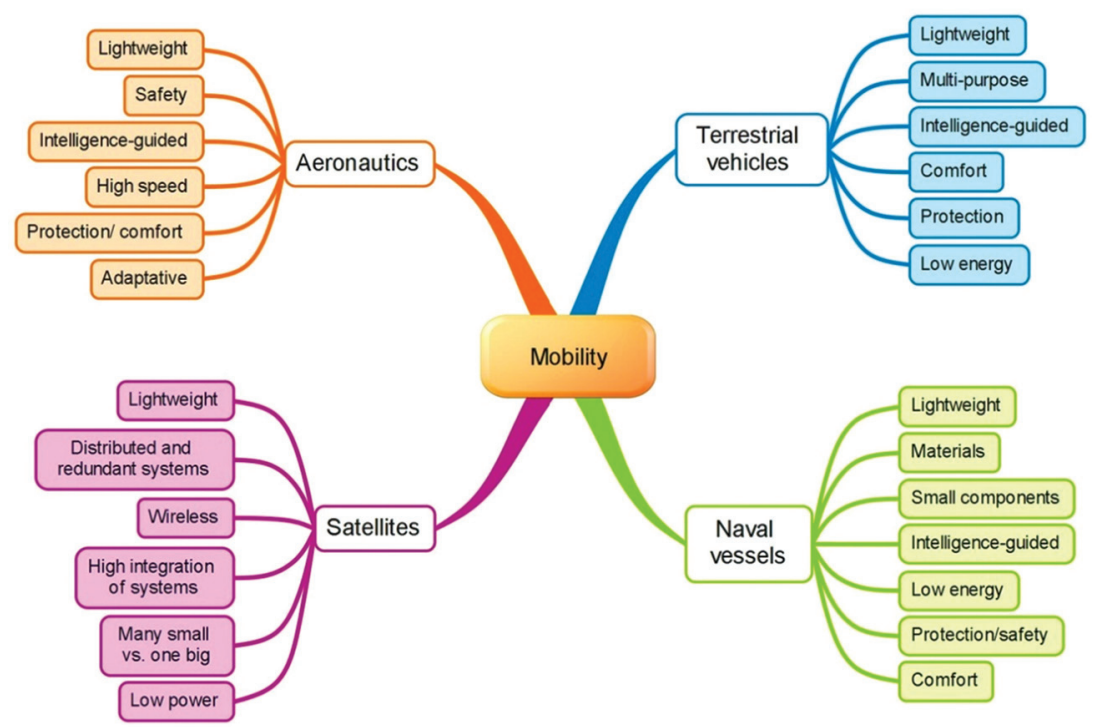

Figure 139. Aspects of military mobility to which nanotechnology can contribute. Created using the data of Simonis and Schilthuizen, Nanotechnology, innovation opportunities for tomorrow's defense, TNO Science and Industry, 2006.

\section{Mobility}

Mobility is an essential element of military tactics. This includes the use of terrestrial vehicles, naval vessels, aeronautics and satellites. Figure 139 indicates the areas in which nanotechnology can be employed to improve military mobility.

Lightweight vehicles can be constructed using nanocomposite plastics to replace metal. Another advantage derived from this is reduction of radar signature.

Providing adequate energy to the vehicle is always an issue. Energy requirements can be reduced by development of low energy consumption vehicles and low power consumption electronic equipment. As discussed in chapter 17, nanotechnology is already contributing to design of energy sources such as batteries, fuel cells, etc.. Nanocoatings are used to reduce wear in engines. The aerodynamic drag of fast vehicles such as planes or missiles can be reduced using nanocoatings and nanocomposites.

In aeronautics, there is a demand for vehicles with small radar and thermal signatures. Nanotechnology can provide radar absorptive coatings and high energy propellants based on nanodispersed aluminum. 


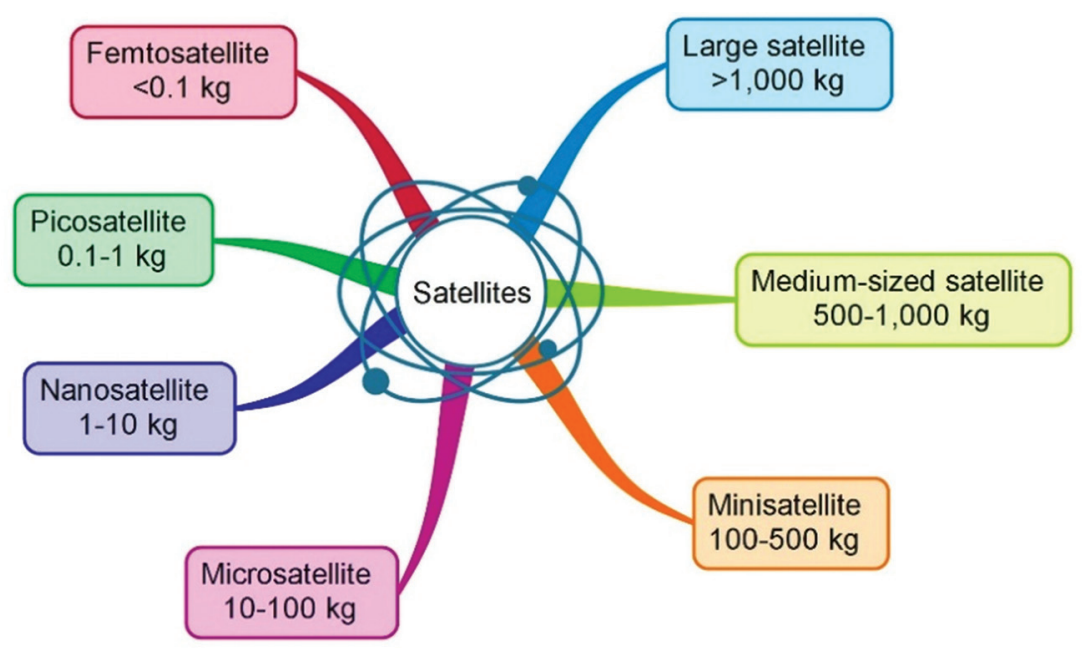

Figure 140. Classification of satellites according to their deployed weights (data from www.daviddarling.info).

Satellites are extensively used for observation and telecommunication. The use of nanocomposites can greatly reduce weight, a critical issue for the equipment carried by satellites. There is an increasing trend to rely on orbiting networks of small satellites rather than a single large satellite. A classification of satellites according to their deployed weight is shown in figure 140.

The nanotechnology based technique of quantum cryptography has a great potential for secure communications. It is based on quantum physics and the entanglement effect. With this technique one knows for sure that no one has read or modified the information before it is received.

\section{Weapons}

Modern weapons can be classified into two main categories: nonlethal and lethal. The goal of nonlethal weapons is to temporarily neutralize an enemy. They are mostly used by the police and security services. Lethal weapons are those capable of producing great bodily harm or death. The goals of weapon development are to make them even more effective while increasing precision, targeting and intelligence.

Nanotechnology enters in several areas of weapon development as is illustrated in figure 141. 


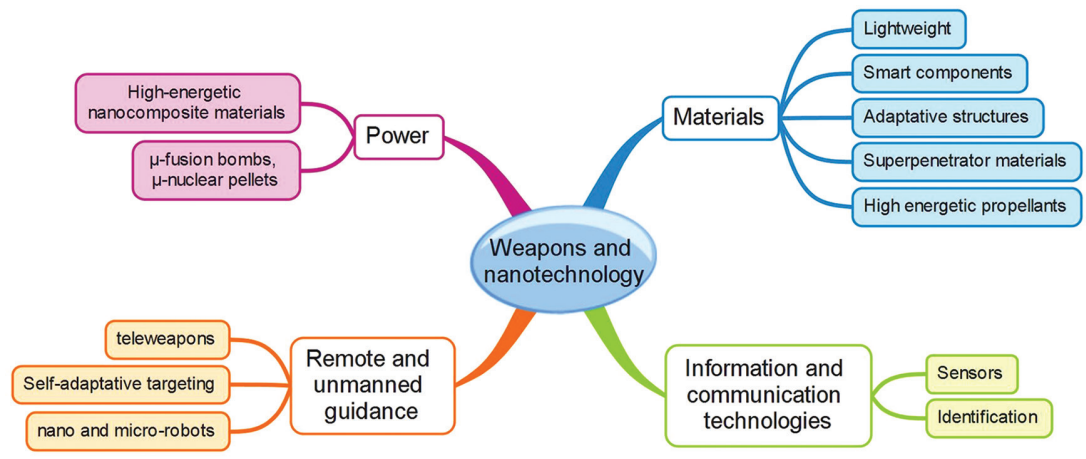

Figure 141. Areas where nanotechnology can improve weapons.

\section{Homeland security}

We live in a dangerous world. Natural disasters such as fires, floods, cyclones, typhoons, tornados or earthquakes can lead to great damage and disorganize societies. Terrorist attacks, executed by fanatics, are difficult to defend against (who, where, how and when?). They create a great sense of insecurity in the population. Anticipating and dealing with these threats and dangers is difficult. Technology can help. Within the technology sector, nanotechnology plays an increasing role.

Mitigating threats requires timely actions. To protect people and equipment threats must be detected, appropriate responses must be made and in the event that damages occur repairs must be made. The different aspects of threat mitigation are illustrated in figure 142.

\section{Detection}

Detection of the threats is essential to prevention. Appropriate sensors and analytical devices can provide early warnings of possible threats and allow intervention in a timely manner. In the event of a successful attack, similar analytical techniques may be employed in forensics investigations of the incident. This can be important in preventing recurrences and in pursuing perpetrators of these incidents as well as the detection of the nature of the products that have been used when it is the case. The main sectors in which analytical detection techniques are applicable are indicated in figure 143.

Releases of toxic chemicals can present serious threats to a community. This is true whether they are accidental or deliberate releases. 


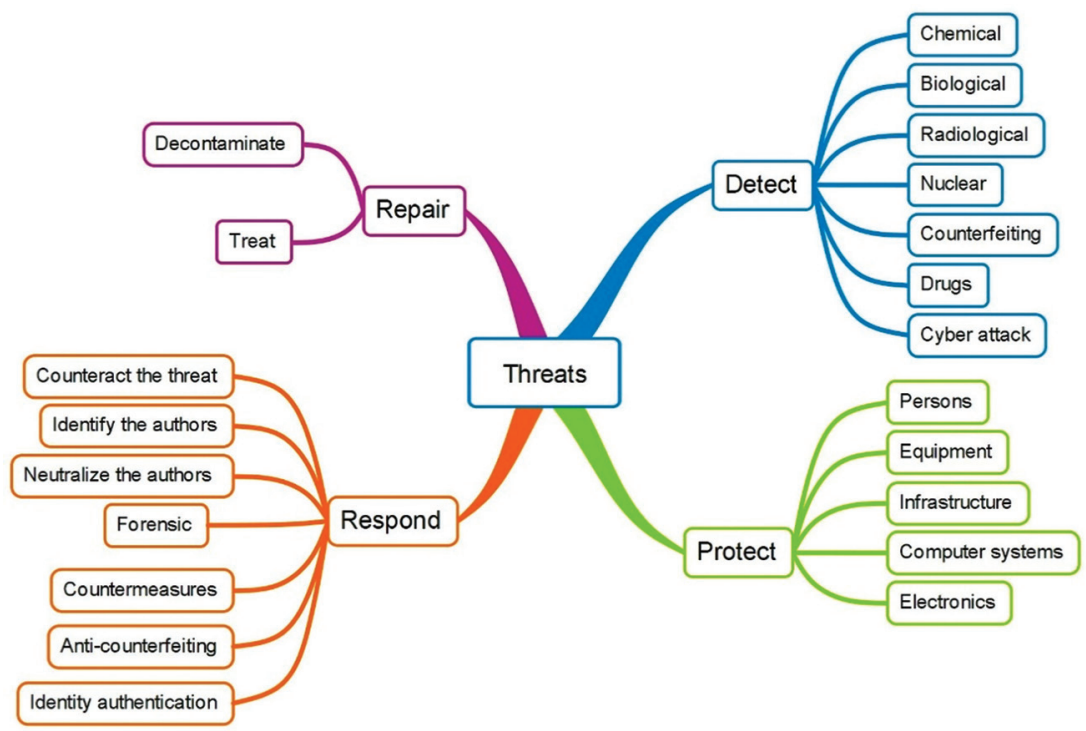

Figure 142. Threat mitigation.

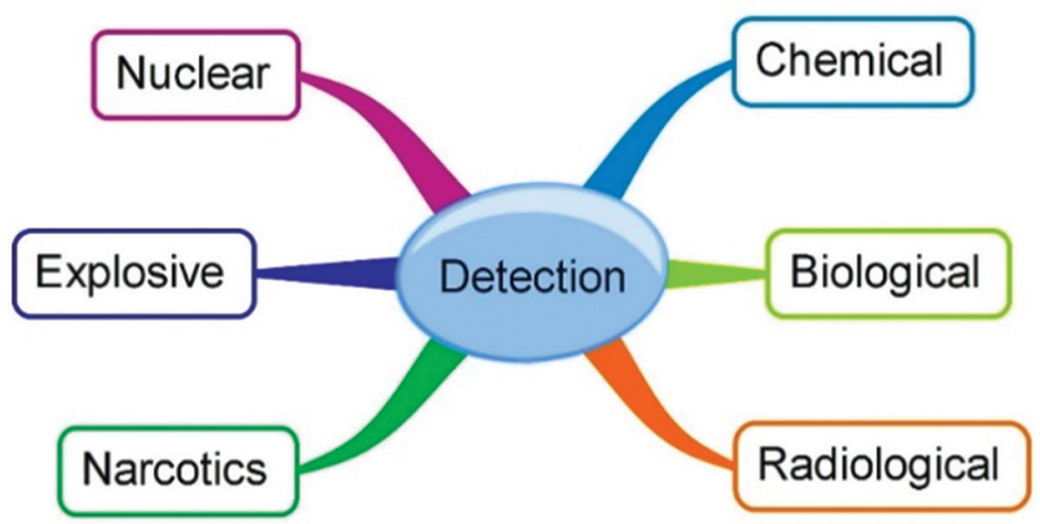

Figure 143. Sectors where detection is required.

The Observatory nanoproject has developed the classification of dangerous toxins presented in Figure 144.

A nerve agent can be sarin, tabun, cyclosarin, etc. Phosgene and chlorine are examples of choking agents. Blister agents include mustard gas or phosgene oxime, commonly known as CX. Cytoxis agents such as ricin or abrin are extremely toxic. In the case of accidents in the chemical industry, a number of harmful agents such as hydrogen cyanide, mercury or phosgene 


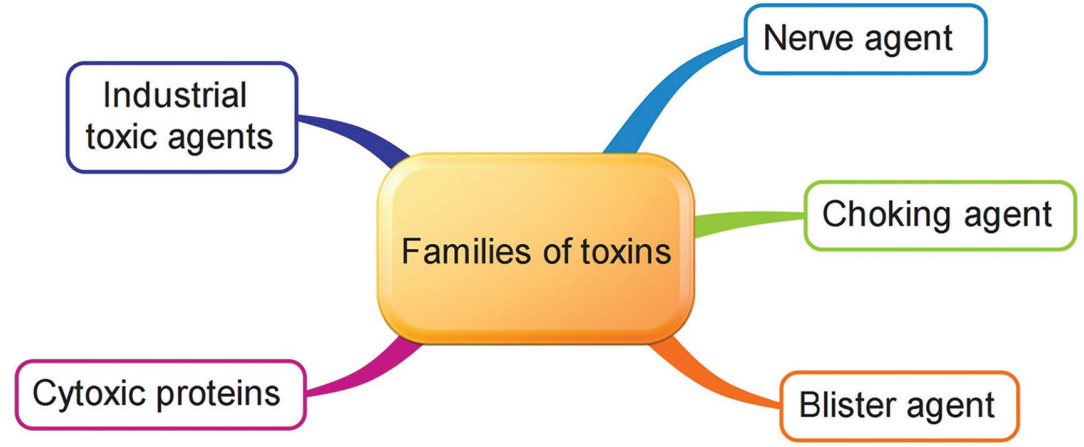

Figure 144. Different families of toxins. Classification from the ObervatoryNano project.

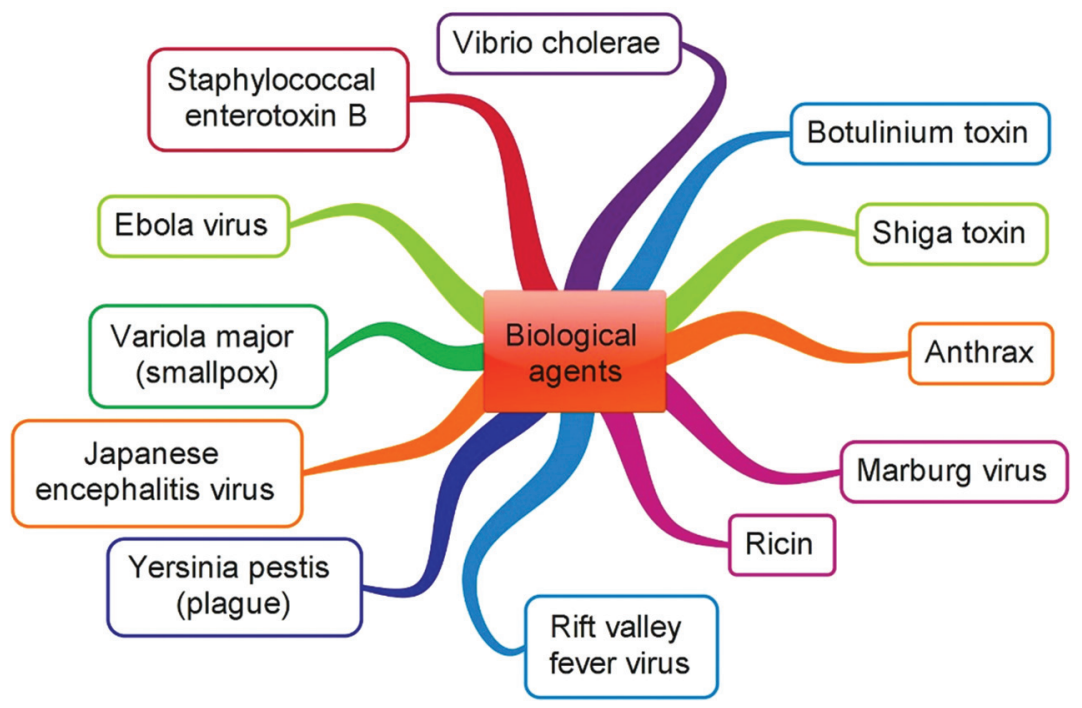

Figure 145. Examples of biological agents that could be used in biological weapons.

might be emitted. High sensitivity, material specific, and cheap sensors are required for these different toxins.

Viruses, bacteria and toxins are biological threats that can be spread among the human population, livestock, crops, food or water supplies in a terrorist attack. Some of the biological agents which can be potentially used as biological weapons are shown in figure 145. Recognition of these agents is based primarily upon nucleic acid and immunological detection techniques but physical and chemical techniques can also be useful. 
Nanotechnology and nanomaterials including nanoparticles, carbon nanotubes, and quantum dots are already being employed in several currently available sensors developed for toxins and biological agents. The small sizes of nanoparticles, nanotubes and nanowires means that the electrical properties of the sensing elements are sensitive to only a few molecules.

Nuclear proliferation and the possibility of misappropriation of a nuclear weapon or dispersal of radioactive matter using radioactive materials and conventional explosives constitute major threats in the modern world. Very sensitive detectors for nuclear materials exist but there is a need to have cheap and portable nuclear detectors. High resolution germanium detectors operating at low temperature are excellent gamma ray detectors but are expensive. Nanowire arrays of detectors are useful for imaging techniques and high counting rates. Alternative low cost detectors based on nanomaterials operating at room temperature can be useful to make quick measurements even if the resolution is not as good.

Detecting explosive materials in cargo, vehicles, luggage, aircraft etc. is a high priority objective of security forces. More than one hundred different types of explosives can be used and it is necessary that they be detected quickly and efficiently. Several detection techniques are employed in this sector. A number of them rely on the fact that absorption of volatile molecules found in the explosive modifies a physical or chemical property of the sensor. Nanocatalysis is often part of the detection process.

Narcotics detection requires cheap, selective, sensitive, fast and small sensors that can be operated under various conditions of temperature and humidity. The techniques utilized are similar to those generally used in chemical detection. The detection of narcotics is also a sector where nanotechnology and nanomaterials are involved. For example, selective membranes of nanomaterials are used to detect fentanyl that is forty time more potent than heroin.

\section{Protection}

Protecting people, infrastructure and equipment during and after explosions or chemical, biological and radiological accidents or attacks requires a variety of measures to be taken.

Some protection can be offered by proper clothing. Protective clothes should be affordable, lightweight and durable. Nanocomposites are 
expected to play an increasing role in this area. Nanofibers have large active areas that can be functionalized in order to provide a better protection against aerosols. Fabrics made out of carbon nanotubes offer a better shielding against explosives and bullets and are lighter than fabrics based on steel. Magnesium oxide nanoparticles loaded into nanofibers turn out to be better protective adsorbents than activated charcoal used today.

Buildings can be destroyed or damaged by explosions, fire, earthquakes, tsunamis, etc. Electronic infrastructure including communications, data processing and control equipment can also be partially or totally destroyed in an electromagnetic attack (E-bomb). Incorporation of nanomaterials in concrete or steel alloys increases the strength of these materials against ballistic projectiles. Carbon nanotubes or inorganic fullerenes can reinforce structures against explosions, or earthquakes. Nanocoatings or nanoadditives can improve the fire resistance of materials. Electromagnetic shielding with conducting polymers or nanocomposites containing conductive polymers can protect electronic equipment against electromagnetic attacks.

\section{Response}

Identification of dangerous individuals and the ability to detect them when they travel around the world is a first response to potential threats. There are several more or less complex methods for authentication of the identities of individuals: fingerprints, DNA, iris, retinal or facial identification, etc. Nanocomposites can be used as a medium to record biometric information.

Response to a security breech includes counter measures such as identification and neutralization of those responsible, minimization of negative effects and taking appropriate actions to prevent reoccurrence. Forensic analysis of evidence at crime scenes has become more and more efficient thanks to new techniques of investigation. Forensics aims to determine the origin and causal links for a crime or a terrorist attack. A number of the techniques employed rely on nanotechnology that enhances the analysis capabilities and give new possibilities to pin down relevant indices that can be used to expose the persons responsible.

As an illustration of a device issued from nanoscience, which has major applications in forensics, figure 146 shows applications of the scanning 


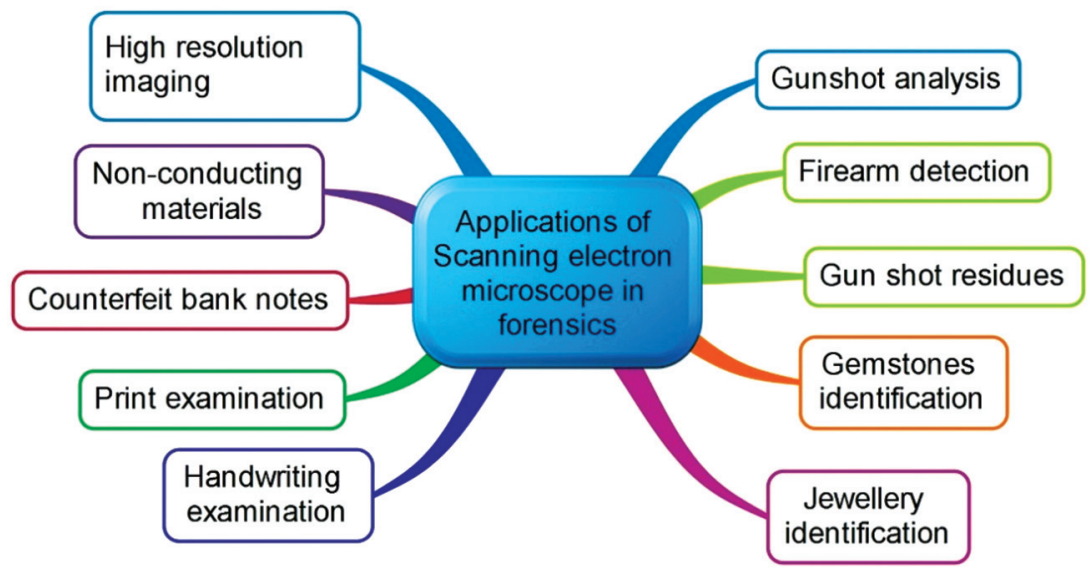

Figure 146. Some applications of scanning electron microscopy in forensics. Examples from OservatoryNano project.

electron microscope. Depending upon the technique used in the microscope, spatial resolutions in the nanometer range can be achieved.

A study by the OECD has estimated that, in 2007, international trade of counterfeit and pirated goods amounted to about 250 billion dollars, representing around $2 \%$ of global trade in that year. Many of the solutions used to protect products and consumers against counterfeiting are based on nanotechnology. Cheap intelligent materials and devices, DNA tags for example, can be used to label products and their packaging. There is an increasing demand to trace products all the way from the manufacturer to the consumer. Positioning and localization techniques based on electronic devices are used to do that. Radio frequency identification tags (RFID) are now widely used to identify objects. Metal nanoparticles or carbon nanotubes improve their operating characteristics.

The main anti-counterfeiting technologies based on nanotechnology are shown in figure 147 together with their areas of application.

\section{Repair}

After a terrorist attack or a natural disaster, repairs must be made. People injured during a chemical, biological, radiological dispersive devices, nuclear and explosive (CBRNE) attacks need medical aid and decontamination. Decontamination is also necessary in the case where the environment has been compromised. 


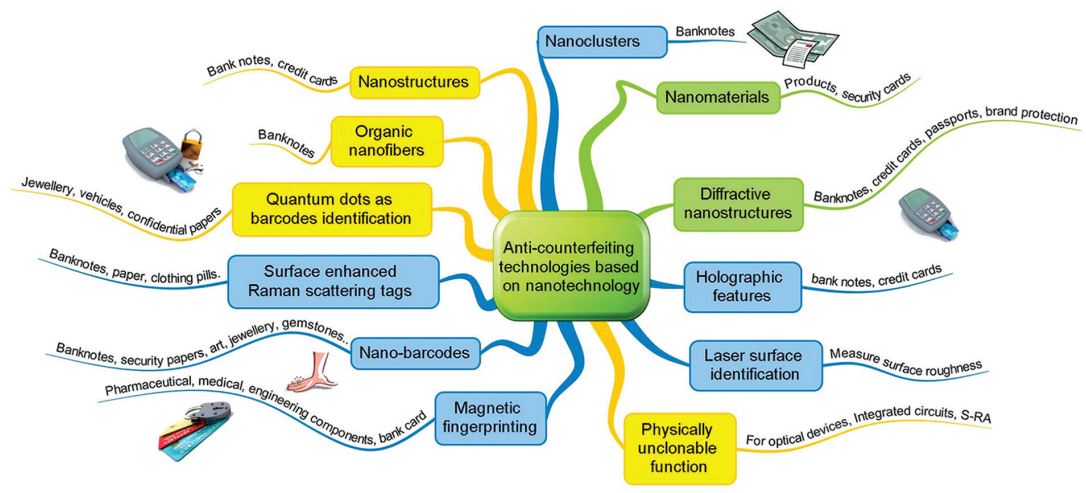

Figure 147. Main anticounterfeiting techniques based on nanotechnology (from the ObservatoryNano project). Techniques which are commercial or pre-commercial are in blue. Techniques at the prototype level are in green. Techniques in the research stage are in yellow.

Nanoparticles and nanomaterials are effective decontamination agents because of their high reactivities and large surface areas. They can be used to decontaminate chemical warfare agents. Nanosized metal oxides of aluminum, titanium and cerium can be used for decontamination and disinfection. Nanocrystals of magnesium oxide can absorb organophosphorous compounds. Their advantage compared to activated charcoal is that they react much faster and destroy the dangerous compound rather than simply physisorb it. Nanoscale powders of magnesium and calcium oxides have antimicrobial properties. They can destroy vegetative cells of Escherichia coli and several bacilli. Other specific nanoparticles are also used to quickly decontaminate objects after bacillus anthracis attacks or Escherichia coli contaminations.

Titanium dioxide $\left(\mathrm{TiO}_{2}\right)$ can be used as a photocatalyst for pollutant destruction. A great advantage is that it is inexpensive and non-toxic. Using $\mathrm{TiO}_{2}$, it is possible to manufacture photocatalytic nanowires that can be transformed into free standing membranes which can be used in filters for gas masks and to block toxic gases. Many other possibilities exist for decontamination based on nanoparticles or nanomaterials. A deeper description of these possibilities can be found in the ObservatoryNano report. 


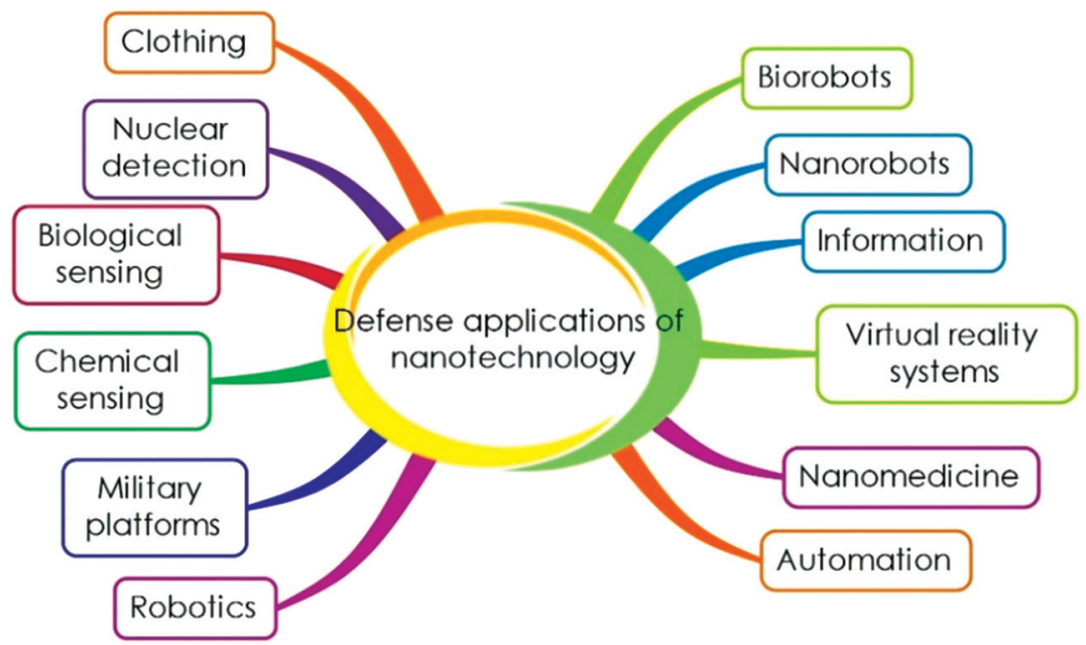

Figure 148. Main sectors in the defense and security domains where nanotechnology can play a role.

\section{Summary}

Nanotechnology is already playing a major role in defense and security applications. This will be amplified in the future and most future military equipment will employ some nanotechnology. An irreversible evolution towards dual-use technologies is observed reflecting cost/benefit analyses and rapid progress in civilian research and development efforts. The keywords in the defense and security domains are smaller, faster, lighter and cheaper. Nanotechnology helps us to address all of these imperatives. Some current and potential applications of nanotechnology to defense or security are summarized in figure 148 .

Homeland security is an overriding concern in modern societies. The primary goal is to anticipate and prevent or at least reduce the impact of terrorist attacks and natural disasters. It is a difficult goal. It relies on detection of threats and a correct treatment of the consequences of attacks or disasters. Technology is a critical tool in this respect. Nanotechnology offers new opportunities to develop cheap, small and smart, fast and selective, sensors. Nanoparticles and nanomaterials are of great help in detecting and decontaminating dangerous or deadly agents. 


\section{Nanotoxicity}

Today nanoparticles and nanomaterials are used in many applications and commonly used in many areas, e.g., health care, electronics, chemistry, foodstuffs, automotive manufacture, energy production, cosmetics, fabrics, sensors, etc. Workers and consumers are often exposed to nanoparticles and nanomaterials. Assessing the risk on human health and the environment generated by these technologies is important but not simple because nanoparticles or nanomaterials can have properties different from those of macroscopic samples of the same materials. They are generally more reactive because they have a much larger surface of interaction.

In vitro and in vivo experiments are carried out to evaluate the toxicity of nanoparticles and nanomaterials. However, there are many controversial data in the literature and conclusions are often difficult to draw. In preparing this chapter, we have relied heavily on several review articles on this subject ${ }^{14}$. We refer the reader to these references to get a deeper insight into the state of the art in nanotoxicology.

\section{Hazard and risk}

Before we go any further it is worth recalling the difference between hazard and risk.

A hazard is a potential source of damage to a person, to equipment or to an organization. For example, when you are biking you can fall from your bicycle. A risk is the possibility that harm can result from a selected action or exposure to a particular hazard. The level of risk can often be quantified as a probability that harm will occur. Low risk means the probability of harm is relatively small. High risk means that the probability is large.

The level of risk depends both on the hazard and on your degree of exposure to the hazard. If you never use a bicycle, you have no chance to fall down. Loosely speaking, the level of risk is a quantity equal to the product of the degree of exposure to the hazard, times the seriousness of the hazard itself. For example, all things being equal, the risk of falling from a bicycle increases when biking more and more. Of course, without hazard, there is no risk.

Actually, zero risk doesn't exist. In any assessment of risk versus reward, the advantages and disadvantages of a given action should be evaluated 


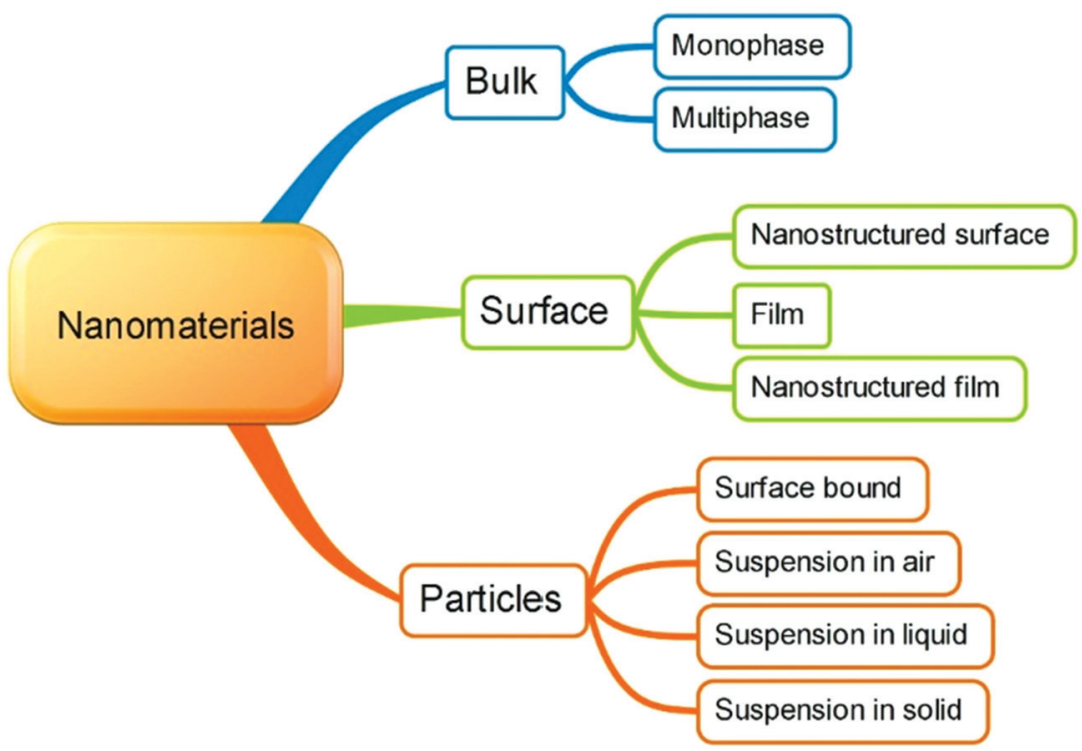

Figure 149. Classification of nanomaterials according to their location in an object.

and understood. This issue exists in most human activities and requires a careful analysis. Effort should be made to reduce risk as much as possible.

\section{Nanomaterials and nanoparticles}

The term nanomaterials encompasses materials composed of nanoparticles and those which are structured on the nanometer scale. A schematic means of classification of nanomaterials is summarized in figure 149. Nanomaterials can form the bulk of the material either as a single phase or as multiple phases (mixtures). Very often nanomaterials can be found on the surface of macroscopic objects providing them with specific properties. This can be a nanofilm deposited or built on a macroscopic surface, or a nanostructuration of the surface. For both bulk and surface nanomaterials, the nanoparticles or nanostructures are bound to the macroscopic object and give novel properties to the object, e.g., enabling photocatalysis to destroy harmful organic chemicals.

When the nanoparticles are embedded in a material which is part of a consumer product, the risk is usually very small for the consumer. Nanoparticles are trapped in the material and can be released only by wear or abrasion.

Nanoparticles can also be disbursed as suspensions in air, liquids or solids. 


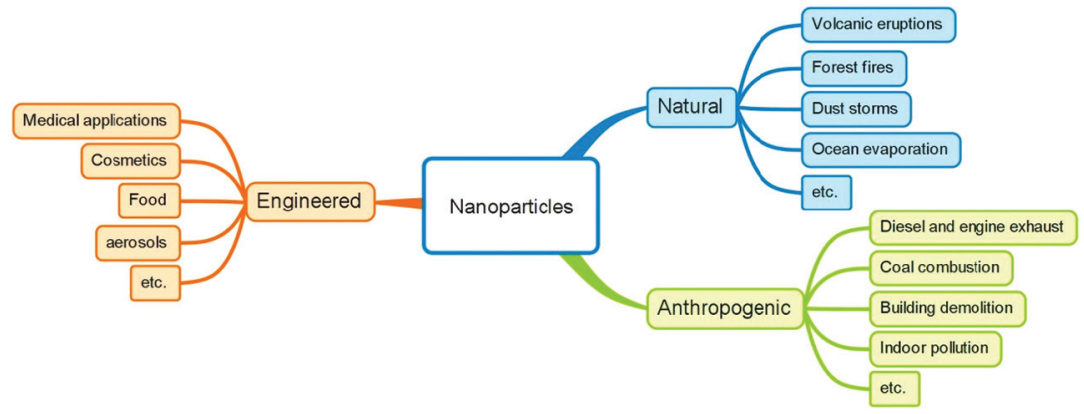

Figure 150. Nanoparticles can be of natural origin or man-made. For man-made nanoparticles one can distinguish between anthropogenic particles which are a byproduct of human activities and engineered nanoparticles which have been purposely manufactured for a specific application. Some domains of the different nanoparticles are indicated for illustration.

\section{Nanoparticle sources}

Nanoparticles can have different origins:

- They can come from natural processes such as volcanic eruptions or forest fires

- They can be emitted during human activities such as combustion of coal or diesel fuel. We shall say that they are from anthropogenic origin: These are man-made but not created on purpose.

- Nanoparticles can also be produced on purpose and used because of their specific properties. This is the case for example of functionalized nanoparticles used in therapeutics, or nanoparticles such as $\mathrm{TiO}_{2}$ used in cosmetics, toothpaste or sun creams. These nanoparticles are of anthropogenic nature. We shall put them in the special category of engineered nanoparticles. Figure 150 shows our classification scheme with a few representative examples of the domains in which they are encountered.

\section{Nanoparticle exposure}

Exposure to certain nanoparticles may have an adverse effect on human health Airborne nanoparticles are certainly those which present the largest potential hazard to human health. Free nanoparticles are usually engineered in such a way that they do not aggregate with each other. If nanoparticles penetrate into some part of the body they can induce health problems. 


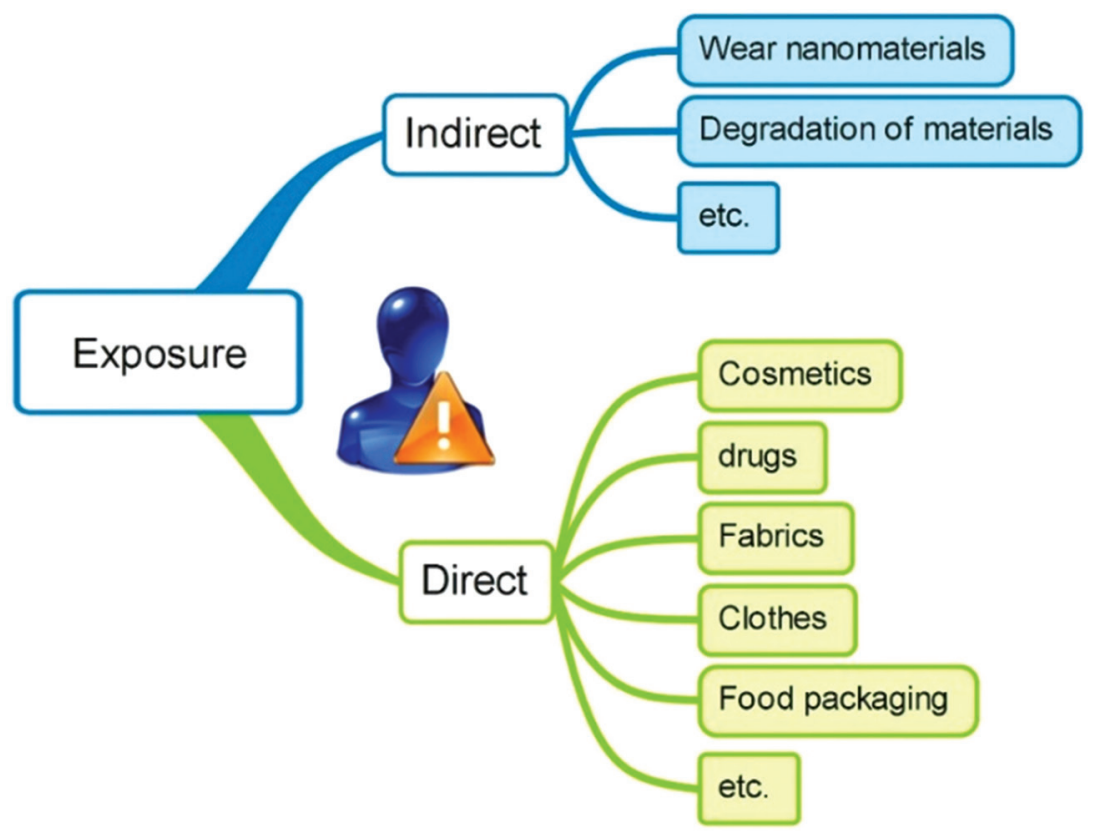

Figure 151. Direct and indirect exposure to nanoparticles.

The exposure to nanoparticles may be direct or indirect (figure 151). There is a direct exposure when the nanoparticles are intended for contact with the consumer. This is true of cosmetics, drugs, fabrics, clothes, food packaging, etc. Indirect refers to non-intended exposures which might occur during the use of an object containing nanomaterials. Such exposures might come from surface coatings of the objects, or from their degradation at the end of their life.

Nanoparticles can enter the human body by several routes. These are displayed in figure 152: inhalation, penetration through the skin or wounds, by ingestion or through the parenteral route (intravenous, intra-arterial, intra-osseous, intramuscular, intrathecal). Since the skin, lungs and the gastro-intestinal tract are in contact with the environment, they are potentially the easiest entry points for nanoparticles.

\section{Toxicity of nanoparticles}

Workers can be faced with high concentrations of nanoparticles during the manufacturing process. Inhalation is a favored route of contamination, but 


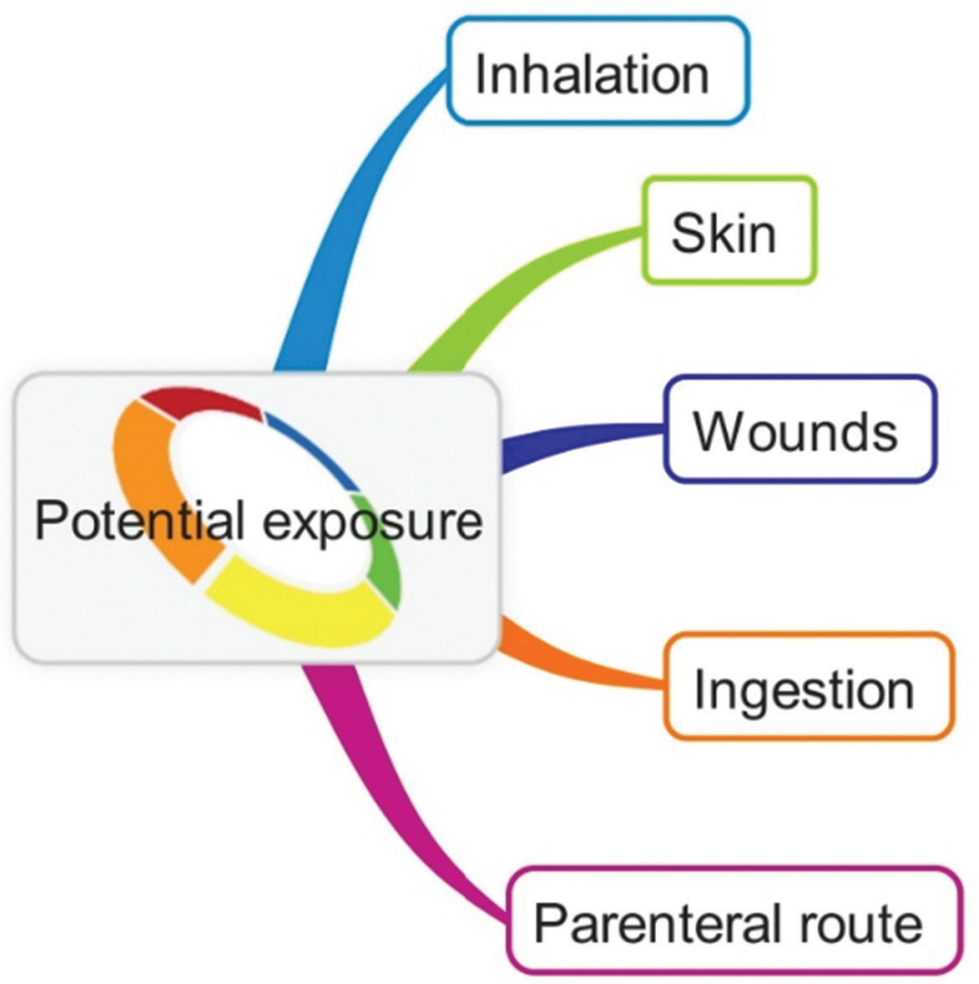

Figure 152. Routes that can be taken by nanoparticles to enter the body.

skin and wound penetration are also efficient pathways for the nanoparticles to penetrate the human body. The workers should be protected against any direct interaction with the nanoparticles. At the manufacturing level it is relatively easy to design an industrial installation to prevent worker contact with nanoparticles using appropriate protective clothing, filtration and ventilation techniques. Product recovery, cleaning, equipment maintenance, packaging processes and storage are also steps leading to potential worker exposure to nanoparticles. At the industrial level and with good standards, this problem can be solved. It is, however, very difficult to control the full life-cycle (production, transport, storage, usage and disposal) of an object containing nanomaterials. There is a risk for consumers that must be evaluated and minimized.

Evaluation of the toxicity of nanoparticles is a complex and difficult problem. Some health disorders may only appear a long time after exposure. Most toxicology studies are carried out in vitro or in vivo on animals or cells and high concentrations of nanoparticles are used. These concentrations do 
not necessarily correspond to those normally encountered. Establishing permissible dose levels is an important imperative that requires deeper studies.

To illustrate the point - there have been toxicity studies of the effect on human cells of iron based magnetic nanoparticles as well as of silver and zinc oxide nanoparticles. A summary of these studies has been published by Auj-E Taqaddas ${ }^{15}$. Magnetic iron nanoparticles have several potential applications in medicine such as contrast agents in diagnostic, targeted drug delivery or hyperthermia. They are considered safe because of their biocompatibility and the fact that an excess of these nanoparticles is eliminated by the liver and the spleen. However, it has been shown in tests on specific breast cancer cells that these nanoparticles can damage the membrane of the cell. The extent of the damage depends on the concentration of nanoparticles, on their sizes and on the contact time. The nanoparticles induce an intracellular stress on the cells that damages DNA and can lead to apoptosis (death) of the cell. It is possible to reduce the toxicity of the nanoparticles by surface coating them, for example with gold or by a proper functionalization of the surface.

Silver nanoparticles are widely used in particular because of their anti-microbial and biocidal activities but they also have interesting properties such as high thermal and electrical conductivities. They are increasingly used, especially in medicine and in fabrics. Because of their toxic properties to organisms, it must be verified that the use of silver nanoparticles is safe for health and the environment.

In the health area, although they have been used for many years, it seems that silver nanoparticles are safe as long as they are used at low dose. The use of silver nanoparticles in fabrics has been approved in many countries. A problem is that fabrics can release some of these nanoparticles into the environment, during washing for example.

The potential diseases that can be associated to exposures to nanoparticle are summarized in figure 153 (Critina Buzea et al). This chart has been deduced from the results of in vivo and in vitro studies. It summarizes possible health problems associated with inhalation, ingestion and contact with nanoparticles. This figure corresponds to the worst situation that can be imagined.

The toxicity of nanoparticles depends on several factors such as their size, shape, composition, functionalization, structure, crystallinity, etc. It also depends on the biological properties of the organism, the pathway followed by the nanoparticles and their nature.

- Inhaled nanoparticles can lead to asthma, bronchitis, emphysema, lung cancer, and neurodegenerative diseases (Parkinson's, Alzheimer...). 


\section{DISEASES ASSOCIATED TO NANOPARTICLE EXPOSURE}

C. Buzea, I. Pacheco, \& K. Robbie, Nanomaterials and nanoparticles: Sources and toxicity, Biointerphases 2 (2007) MRI7-MR7I

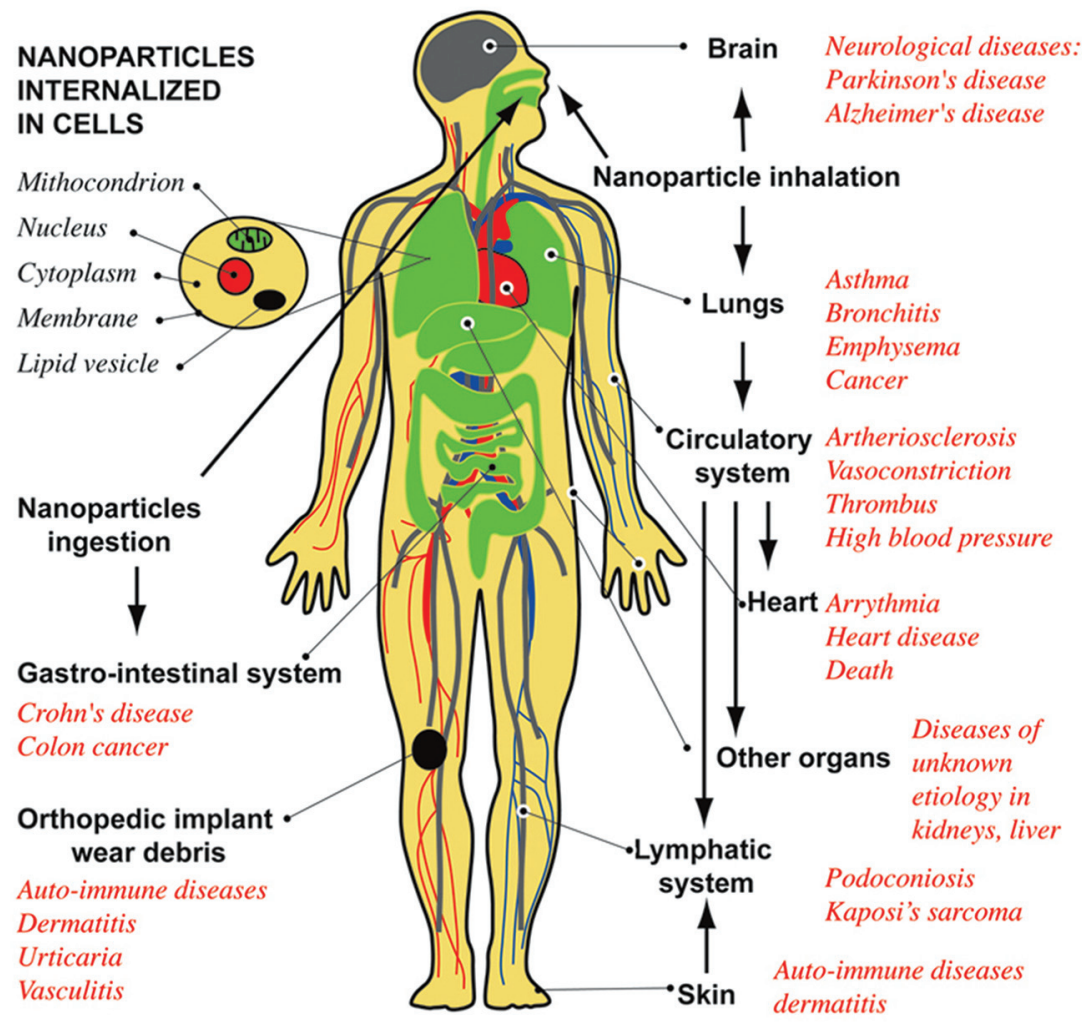

Figure 153. Pathways of exposure to nanoparticles and associated diseases as suggested by epidemiological, in vivo and in vitro studies. Image from Wikimedia Commons (http://commons. wikimedia.org), Cristina Buzea.

- In the gastrointestinal tract, nanoparticle ingestion can lead to Crohn's disease and colon cancer.

- Nanoparticles can also enter into the circulatory system with the possibility of inducing arteriosclerosis, blood clots, arrhythmia, heart diseases and possibly cardiac death.

Translocation to other organs (liver, spleen, brain...) may induce other diseases. Furthermore, some nanoparticles are suspected of inducing autoimmune diseases.

This description of adverse health effects can be scary but relates to only a small number of the natural nanoparticles found in the environment or 
those which are man-made. Many nanoparticles are non-toxic and the consequences on health care shown in figure 153 do not apply. It is also possible, starting from a nanoparticle that is initially toxic, to modify it to make it non-toxic. Furthermore, nanoparticles can aggregate locally, producing larger sized particles that are less dangerous for health.

In the following three sections we discuss some of the health issues associated with the three different classes of nanoparticles identified in Figure 150.

\section{Natural nanoparticles}

There are naturally occurring nanoparticles (1-10o $\mathrm{nm}$ in size). These nanoparticles, not produced by human activities, are mostly found in hydrous environments. Produced by forest fires, volcanic eruptions, dust storms or similar events. These nanoparticles may be found in streams, can be carried by the wind and dispersed into the environment. They are present in large numbers in each breath we take and in our drinking water.

Less violent natural processes can also produce nanoparticles ( $\mathrm{ZnS}$, $\mathrm{Fe}_{3} \mathrm{O}_{4}$, metal sulfides...). For example, zinc sulfide (ZnS) nanoparticles are formed by microbial action. More generally, other natural processes, for instance, drainage in acid mine sites are able to produce mineral nanoparticles. Generally, most natural nanoparticles have a low toxicity.

At the global level, most atmospheric aerosols are produced by wind erosion. Dust storms can travel over long distances. For example, the particles of dust storms occurring in the Gobi Desert can reach North America. The size of the particles transported is generally between $100 \mathrm{~nm}$ and several microns. Particles between $100 \mathrm{~nm}$ and $200 \mathrm{~nm}$ can be found at concentration around 1,500 particles $/ \mathrm{cm}^{3}$. These airborne dust particles can induce health problems with people subject to asthma and emphysema. The lung tissue can also be affected thanks to iron and other metal particles present in the dust.

Ashes and gases containing particles with sizes ranging from the nanoscale to the microscale emitted in volcanic eruptions, cause respiratory problems, eye and skin irritation. Traveling to very high altitudes, they spread over the world and can affect areas of the planet for years following an eruption. Populations living barefoot in areas with a volcanic soil are affected by serious diseases (podoconiosis, lymphedema, Kaposi's sarcoma, etc.) probably coming from the absorption through the soles of nano and microparticles coming from the soil. 
A large quantity of aerosols is emitted from the seas and oceans. Sea salt particles with dimensions from $100 \mathrm{~nm}$ to several microns can be found in these aerosols. Unlike other kinds of aerosols, they have a positive effect on health and can relieve some respiratory problems.

Nano-organisms such as bacteria, viruses and other organisms can have an impact on human health. They are probably the cause of some neurodegenerative diseases. Nanobacteria could be involved in diseases linked to calcification such as artery plaque, aortic aneurysm, renal stone formation, prostatitis, ovarian and breast tumors, etc.

Gas molecules and viruses can have a size in the 1-100 nm range. For example, the HIV (human immunodeficiency virus) is about $100 \mathrm{~nm}$ diameter. Molecules and viruses can be present in the environment, especially in the air, but also in water. Viruses interact with biological systems and exploit cellular processes to replicate themselves. Some viruses or nanobacteria have seriously adverse health effects on people. Some chronic diseases that were thought to come from lifestyle or genetic factors are actually due to viruses. For example, cervical cancer is due to the Papilloma virus and the nasopharyngeal cancer comes from the Epstein-Barr virus.

The effect on health of inert nanoparticles present in the environment should not underestimated compared to viruses despite the fact that they do not have the ability to take control of a cell and replicate themselves. Their very small sizes give them the ability to enter the organism and translocate within it. In the worst case, within the organism, these particles can cross physiological barriers (air-blood barrier in the lungs, blood-brain barrier or materno-foetal barrier) and travel through the circulatory system (blood circulatory, lymphatic system). Skin, lungs and the gastro-intestinal tract are in contact with the environment. They are potentially good entry points for nanoparticles.

Some nanoparticles can penetrate inside cells and induce damage. They can modify processes of the cell. Some diseases may result from the perturbation of the cell operation. For example, cancer, which is an uncontrolled proliferation of cells, can be induced as can neurodegenerative diseases which are due to a premature death of cells. Autoimmune diseases can also be triggered by modifications of the cell functions.

The outer membrane of a cell, which is a phospholipid bilayer, can be affected directly by a nanoparticle, leading to a disruption of the cell membrane such as a nanohole punched into the membrane. The membrane can also be affected indirectly, for example by oxidative or surfactant damage, or damaged by toxic ions. The interaction of a nanoparticle with a membrane 
depends very much on the properties of its surface. Inside the cell, nanoparticles can perturb the cell's constituents. In the nucleus, the DNA can be damaged. The lysosome and mitochondria can also be damaged and there can be a lipid peroxidation in the vesicles.

\section{Anthropogenic nanoparticles}

There are also nanoparticles present in the environment which are not produced on purpose, but produced in normal human activities. These are called anthropogenic nanoparticles. They result from industrial activities such as emission from the exhaust pipes of automobiles and trucks, the production of electricity using coal plants or in the smoke from cigarettes. In large cities it is common to have a few hundred million nanoparticles per liter of air. In discussing this category, we exclude engineered nanoparticles which have been manufactured on purpose for a specific application. These are treated in the following section.

Vehicles, especially those using diesel engines, are the main source of particles (micro and nano) in urban areas. Most of the particles emitted from the exhaust pipes have sizes in the range of $20-60 \mathrm{~nm}$ for diesel engines and 20-130 $\mathrm{nm}$ for gasoline engines. These particles are almost spherical. However, in the case of diesel engines, carbon nanotubes and fibers have been found as byproducts of the combustion. Since their shapes are very similar to those of asbestos fibers, there is a non-negligible probability that they could be carcinogens. Air pollution from transportation exhaust is a serious health problem and there is a correlation between the concentration of nanoparticles near the roads or highways and an increased rate of cardiopulmonary mortality for those people living very close to them. Nanoparticles increase the risk of cardiovascular events.

Burning coal in power plants produces carbon and sulfide particulates and, surprisingly, uranium dioxide $\left(\mathrm{UO}_{2}\right)$ nanoparticles. Actually, if no efficient filter is used in the exhaust chimney, the radioactivity emitted by burning coal is larger (by a factor which can be of the order of one hundred, depending upon the origin of the coal used) than that emitted from an operating nuclear plant. A good filter for the smoke emitted by a coal plant can become a notably radioactive object.

Tobacco smoke is a complex mixture containing more than 100,000 compounds. Many of these are health hazards. It also contains particles with sizes ranging from $10 \mathrm{~nm}$ to $700 \mathrm{~nm}$, the average being around $150 \mathrm{~nm}$. 
Tobacco smoke greatly increases the risks of developing chronic respiratory illnesses, lung, pancreatic or nasal cancers, and cardiovascular diseases.

Particles are also emitted in the demolition of large buildings. Some of them are toxic, especially when old buildings are demolished because they can contain asbestos fibers or lead. The dust cloud generated during demolition can travel tens of kilometers from the demolition site. Following the attack on the World Trade Center, on September 2001, many first responders suffered respiratory problems. More than 5,000 cancers are attributed to that attack.

Indoor pollution is notably greater than outdoor pollution. Several inside activities create nanoparticles: cooking, smoking, cleaning, etc. Nanoparticles are also produced by living organisms: skin particles, dust mite droppings, etc. Since many people now live more indoors than outdoors, indoor pollution is a real issue.

In some countries in Asia or Africa, indoor smoke emitted from the use of coal, biomass, wood and other fuels is responsible for the deaths of about 1.6 million people per year, half of them children under five years old.

\section{Engineered nanoparticles}

Many kinds of engineered nanoparticles are now produced, some of them in large quantities. Tens of thousands of tons of silica, alumina, black carbon, titanium dioxide nanoparticles and nanoclays are produced every year. Hundreds of tons of nanoparticles of metal, ceramic-metal, rare earth and carbon nanotubes are also produced yearly. Other nanoparticles are produced in smaller quantities. In contrast to the anthropogenic nanoparticles that we discussed previously, engineered nanoparticles are designed to satisfy specific functions. For that they must remain stable, at least until used. Most of these nanoparticles, around $45 \%$, are used in industrial materials while about $3 \%$ are reserved for medical applications. The increasing use of nanoparticles in medical applications demands that they be made as safe as possible. The design of the surfaces of nanoparticles used in drug delivery to increase uptake into cells is an important factor in assuring safety.

Cosmetics is an area where engineered nanoparticles are extensively used. Actually the first use of nanoparticles in cosmetics can be traced back to the black soot and mineral powders used in ancient Egypt. Today nanoparticles with specific optical properties or functions such as antioxidant, peptide or nutrient delivery are employed in creams, lipsticks, toothpastes, 
shampoos, hair conditioners, etc. The advantage of nanoparticles is that they can easily penetrate deeply into the protective layers of the skin. Functionalized fullerenes can now be found in creams because of their supposed free-radical scavenging properties. Alumina nanopowder is also used in cosmetics because it is supposed to reduce small wrinkles.

Very stable titanium dioxide $\left(\mathrm{TiO}_{2}\right)$ nano or microparticles are also widely used in many consumer products: sunscreens, cosmetic creams, food colorants, toothpastes, pharmaceuticals, etc. $\mathrm{TiO}_{2}$ is widely used as a white pigment because of its brightness and high refractive index. About four million tons of this pigment is produced every year worldwide. That represents about $70 \%$ of the total amount of pigments produced in the world. Surprisingly, it can be used to whiten skim milk.

$\mathrm{TiO}_{2}$ nanoparticles are anticorrosive and have photocatalytic properties. This photocatalytic effect is important in several applications where selfcleaning and antifogging properties are needed: self-cleaning windows, tiles, textiles, and antifogging car-mirrors.

$\mathrm{TiO}_{2}$ particles with diameters larger than $100 \mathrm{~nm}$ are considered to be biologically safe for humans and animals. It seems almost sure that $\mathrm{TiO}_{2}$ nanoparticles cannot penetrate the intact skin into the human body. On the other hand, intravenous injection of large quantities of $\mathrm{TiO}_{2}$ nanoparticles can induce pathological lesions of the liver, spleen, kidneys and brain.

Silver nanoparticles have the ability to kill bacteria and microorganisms. Because of this property, they are widely used to impregnate consumer products such as socks, underwear, bedding, sportswear, toys, wound dressing, etc. During use and laundering, silver nanoparticles can be released into the environment, especially in wastewater. Silver nanoparticles are also used in medicine. As for many other nanoparticles, they can cause oxidative stress of cells. The toxicity of silver nanoparticles has been investigated in special cases but there are still large uncertainties to be resolved.

Fullerenes and carbon nanotubes, both single wall and multiwall, are now used in several applications. Published work in the scientific literature is ambiguous because some indicate that carbon nanotubes are highly toxic while others indicate that they not. In vivo studies on animals have been carried out. The toxicity depends upon the length of the nanotube and on its functionalization. Often the toxicity results obtained cannot be easily related to carbon nanotube usage because the usage conditions are different and the carbon nanotubes are different. Further studies are required. 


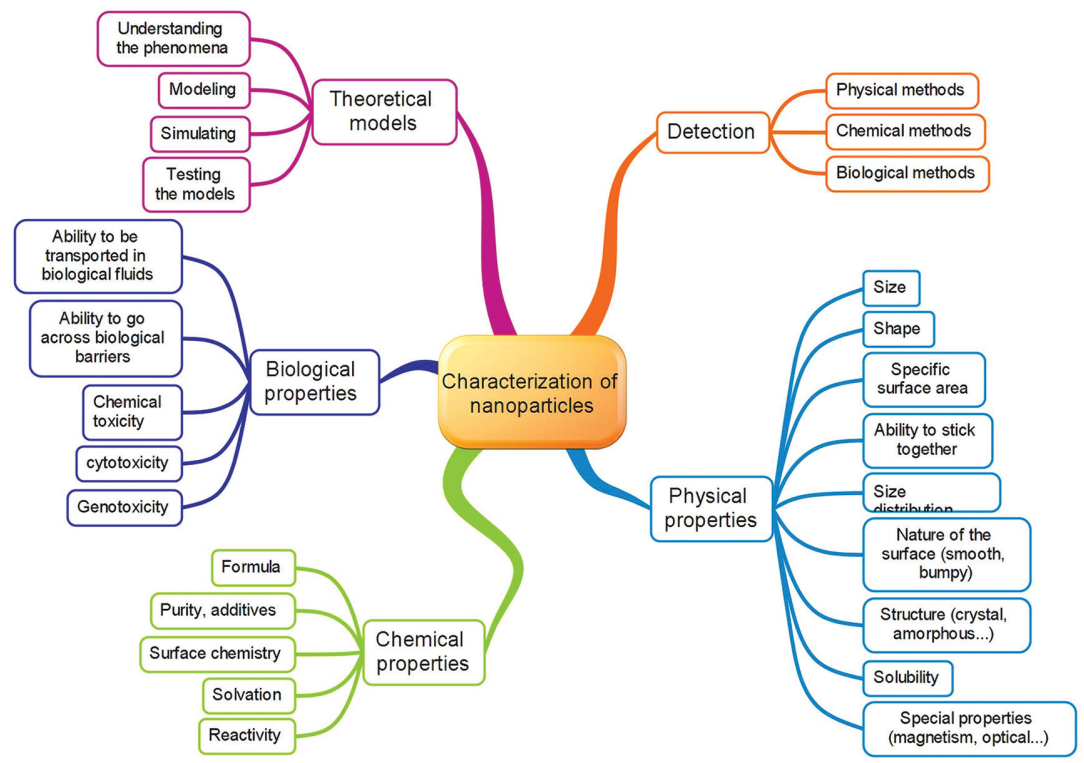

Figure 154. Main areas of investigation to the determination of the toxicities of nanomaterials and nanoparticles.

\section{Summary}

Humans have been exposed to nanoparticles throughout human history. Not all the nanoparticles with which people are in contact lead to serious adverse health effects. It even turns out that some nanoparticles are good for health. This the case for those generated from evaporation of the seas.

The industrial revolution, with its increased combustion of fossil fuels, greatly boosted concentrations of man-made nanoparticles in large cities as well as in many other parts of the world. Many of these have adverse health effects.

The wide-spread use of engineered nanomaterials and nanoparticles adds new risks that must be carefully investigated and balanced with the advantages obtained by their use. While the manufacturing of nanomaterials can be accomplished in working conditions that are safe for the workers and the environment, little is known about long-term exposure of consumers to low concentrations of engineered nanoparticles because it is a rather novel domain. The problem is complex. The interaction mechanisms between engineered nanoparticles and living systems are far from being completely understood. Many parameters are involved and not only the mass or concentration of the nanoparticles. Several aspects have to be 
investigated to get a better understanding of nanotoxicity. Certainly long term epidemiological studies are needed.

The toxicity limits of bulk materials are usually specified in mass units. This parameter is sufficient to characterize the hazard of a material at macroscopic scale. For example, we know that absorbing 100-200 mg of potassium cyanide will kill a normal person. However, for nanoparticles, the mass is not the only relevant parameter. The adverse health effects of nanoparticles depend on many other parameters such as the surface to volume ratio, the shape, the composition, etc. Generally, the smaller the nanoparticle the more toxic it is. However, each situation can be different and nanotoxicology studies have to be carried out or modeled using relevant experimental results. Last but not least, the translocation of nanoparticles inside the body and the interaction with cells are important subjects that need to be fully understood. Since nanomaterials and nanoparticles have different properties from bulk materials, the results of toxicity studies done on bulk materials at the macroscopic scale are not sufficient to describe their toxicities The main areas of investigation relevant to the appropriate determination of the toxicities of nanomaterials and nanoparticles are presented in figure 154 . 



\section{Notes}

\section{Nanoscience and nanotechnology}

1. An adatom is an additional atom lying on the surface of a crystal. This word comes from the contraction of the expression "adsorbed atom". An adatom can be viewed as the opposite of a surface vacancy in which an atom has disappeared.

\section{The Quantum world}

2. Consider for example an operator represented by a square matrix $A$. If we transpose the matrix and take the complex conjugate of all the elements of

the matrix we get a new matrix $B$. If $A$ is equal to $B$, the matrix is said to be Hermitian. For example, $A=\left(\begin{array}{cc}1 & i \\ -i & -1\end{array}\right)$ is a Hermitian matrix. Indeed, the transpose of $A$ is $\left(\begin{array}{cc}1 & -i \\ i & -1\end{array}\right)$ and the complex conjugate of this new matrix is $B=\left(\begin{array}{cc}1 & i \\ -i & -1\end{array}\right)=A$.

3. A function is an eigenvalue of an operator if the result of applying the operator on the function is proportional to the function. The coefficient of proportionality is just the eigenvalue. If $\hat{A} . f(x)=a f(x)$, then $f(x)$ is an eigenfunction of the operator $\hat{A}$ and the number $\mathbf{a}$ is the eigenvalue.

4. If two operators $\mathbf{A}$ and $\mathbf{B}$ commute, the order in which they are applied is not relevant, i.e., $\mathbf{A B}=\mathbf{B A}$. Non-commutivity, $\mathbf{A B} \neq \mathbf{B} \mathbf{A}$, exists if the answer obtained by applying $\mathbf{B}$ first and then $\mathbf{A}$ is different from that obtained if we first apply $\mathbf{A}$ and then $\mathbf{B}$.

\section{The mesoscopic world}

5. A polar molecule is a molecule in which the center of positive charge does not coincide with the center of negative charge. Such polar species are said to have permanent dipole moments. Because of the charge separation they can interact electrostatically with other species having dipole moments. The water molecule is a common example of a polar molecule. There are also more complicated polar molecules characterized by more complicated charge distributions than a two center dipole distribution.

6. The boiling point of water is $100^{\circ} \mathrm{C}$ while the boiling point of $\mathrm{H}_{2} \mathrm{~S}$ is $-60^{\circ} \mathrm{C}$ 
7. Historically, the first successful description of the hydrogen atom, the Bohr Model, was made using classical mechanics to describe the motion of the electron but applying quantization rules allowing only certain trajectories to exist. It was supposed that only classical orbits with a length equal to an integer number times the de Broglie wavelength of the electron were allowed.

\section{King carbon}

8. Graphite is the best known example of a Van der Waals solid. In a Van der Waals solid, there is a strong in-plane bonding between the atoms and a weak Van der Waals interplanar interaction. Many layered materials have this structure.

\section{Health Diagnostics}

9. $\quad$ PLA (Polylactic acid) is a biodegradable polymer used in food packaging (mineral water, fruits and vegetables, bags, eggs ...). It is also used in surgery where suturing is performed with biodegradable polymers which can be dissolved with water or under the action of enzymes.

\section{Regenerative medicine}

10. A review can be found in S. Vahabi and F. Mardanifar, Beheshti Univ. Dent. J. 2014; $32(3): 165^{-1} 76$

\section{Nanocatalysis}

11. Manipulation of Nanoscale Materials: An Introduction to Nanoarchitectonics, K. Ariga, H. Kroto and P. O'Brien, editors, RSC nanoscience \& nanotechnology $\mathrm{n}^{\circ} 24,2012$.

\section{Energy production}

12. The toe (ton oil equivalent) is a unit used to compare different kinds of energy. 1 Gtoe (gigatoe) mean a billion of toe. 


\section{Housing}

13. A III-V semiconductor is a compound combining an element of the column III of the periodic table (essentially $\mathrm{Al}, \mathrm{Ga}, \mathrm{In}$ ) with an element of column $\mathrm{V}$ (essentially N, P, As Sb). Example of III-V semiconductors compounds are AsGA, InP, GaP or GaN.

\section{Nanotoxicity}

14. Nanomaterials and nanoparticles: sources and toxicity, C. Buzea, I. P. Blandino and K. Robbie, Biointerphases Vol.2, Issue 4 (2007) pages MR17-MR172. Toxicology of nanoparticles, A. Elsaesser and C. V. Howard, Advanced Drug Delivery Reviews 64 (2012) 129-137

Nanotechnology - Health and environmental Impacts: A Review, S. H. Khan, M. H. Fulekar and B. Pathak, J. Environ.Nanotechnol. Vol. 4, $\mathrm{n}^{\circ} 3$ (2015) pages $55^{-72}$

15. Journal of Multidisciplinary Engineering Science Studies, 2015 


\section{List of illustrations' copyright and licensing}

For all figures in this book permission for reproduction has been obtained by the authors. Figures not mentioned in this list were originally made by the authors.

3. Marie-Lan Nguyen (2011) / Wikimedia Commons / CC-BY-2.5

5. Courtesy of CEA/LETI (France). Permission for reproduction was obtained by the authors

7. Image originally created by IBM Corporation. Permission for reproduction was obtained by the authors

9. user:OverlordQ / Wikimedia Commons / Public Domain

10. Courtesy of IBM company. Permission for reproduction was obtained by the authors

11. J.A. Stroscio, R.J. Celotta, S.R. Blankenship and F.M. Hess / Wikimedia Commons / Public Domain

31. Edward Pleshakov / Wikimedia Commons / Public Domain

32. user:Freundchen / Wikimedia Commons / Public Domain

34. user:Chem538wıogrp1o / Wikimedia Commons / Public Domain

35. user:Skydivermonkey / Wikimedia Commons / Public Domain

36. user:Sephirothazule / Wikimedia Commons / Public Domain

37. user:Chem538wiogrp6 / Wikimedia Commons / Public Domain

39. Robert Michniewicz / Wikimedia Commons / CC-BY-3.o

41. user:ArchiKat / Wikimedia Commons / CC-BY-3.o

42. William Thielicke / Wikimedia Commons / CC-BY-3.o

43. Bjørn Christian Tørrissen / Wikimedia Commons / CC-BY-3.o

44. Oskar Gellerbrant / Wikimedia Commons / CC-BY-3.o

45. Chris Parfitt / Wikimedia Commons / CC-BY-2.o

5o. Courtesy of CEA/LETI (France). Permission for reproduction was obtained by the authors

61. user:J_Alves / Wikimedia Commons / Public Domain

62. Nanotube image courtesy of CEA/LETI (France). Permission for reproduction was obtained by the authors

63. Image courtesy of CEA/LETI (France). Permission for reproduction was obtained by the authors

66. Image courtesy of CEA/LETI (France). Permission for reproduction was obtained by the authors 
7o. Grieshaber, D.; MacKenzie, R.; Vörös, J.; Reimhult, E. / Electrochemical Biosensors - Sensor Principles and Architectures. Sensors 2008, 8, 1400-1458 / CC-BY-3.0

72. Small images shown are courtesy of CEA/LETI (France) (France) Permission for reproduction was obtained by the authors

75. Image courtesy of CEA/LETI (France). Permission for reproduction was obtained by the authors

79. user:PDPics / Pixabay / Public Domain CCo

9o. Image courtesy of CEA/LETI (France). Permission for reproduction was obtained by the authors

10o. Courtesy of C. Ngô and H. Ngô, Physique des semiconducteurs, Dunod, 4th edition, 2012. Permission for reproduction was obtained by the authors.

102. Image courtesy of CEA/LETI (France), Clefs CEA $n^{\circ} 5^{2}$. Permission for reproduction was obtained by the authors 104. Image courtesy of CEA/LETI (France), Clef CEA $n^{\circ} 5^{2}$. Permission for reproduction was obtained by the authors

105. Image courtesy of CEA/LETI (France), Clefs CEA n ${ }^{\circ} 5$. Permission for reproduction was obtained by the authors

115. Image courtesy of CEA/LETI (France), Clef CEA $n^{\circ} 5^{2}$ Permission for reproduction was obtained by the authors

134. Inspired by the work of White et al. published in Nature 409, 2001.

135. user:Elcap / Wikimedia Commons / Public Domain

153. Cristina Buzea / Wikimedia Commons / Public Domain 


\section{Short bibliography}

Ball P., Made to Measure: New Materials for the $27^{\text {st }}$ Century, Princeton University Press, 1999.

Booker R. and Boysen E., Nanotechnology for Dummies, Wiley, 2005.

Booker R. and Muir N.C., Nanotechnology for Dummies, Wiley, 2005.

Borisenko V. and Ossicini S., What is What in the Nanoworld, Wiley-VCH, 2012.

Bushan E. Editor, Springer Handbook of Nanotechnology, Springer, 2010.

Dosh H. and Van de Voorde M. Editors, GENNESYS White Paper, Max-Planck-Institut für

Metallforschung, 2009.

Edwards S.A., The Nanotech Pionners, Wiley-VCH, 2006.

Feldman M.Editor, Nanolithography: The Art of fabricating Nanoelectronic and Nanophotonic

Devices and Systems, Woodhead Publishing, 2014.

Lindsay S.M., Introduction to Nanoscience, OUP Oxford, 2009.

Ngô C. and Van de Voorde, Nanotechnology in a Nutshell, From simple to complex Systems,

Atlantis Press, 2014.

Pradeep T., Nano, the Essentials, Understanding Nanosciences and Nanotechnology, Tata

McGraw-Hill Publishing Company Limited, 2007.

Ramsden J.J., Nanotechnology, an Introduction, Elsevier, 2016.

Rocco M.C., Mirkin C.A. and Hersam M.C., Nanotechnology Research Directions for Societal Needs in 2020: Retrospective and Outlook, Springer, 2011.

Stepanova M., Nanofabrication, Techniques and principles, S. Dew editors, Springer, 2012.

Turton R., The Quantum Dot: a Journey into the Future of Microelectronics, W.H.Freeman and

Company Limited, 1995.

Wolf E.L., Nanophysics and Nanotechnology: An Introduction to Modern Concepts in Nanoscience, Wiley-VCH, 2015 . 